\title{
APPLICABILITY OF THE NORMALIZED DIFFERENCE VEGETATION INDEX IN INDEX-BASED CROP INSURANCE DESIGN
}

\author{
A Thesis \\ Presented to the Faculty of the Graduate School \\ of Cornell University \\ In Partial Fulfillment of the Requirements for the Degree of \\ Master of Science
}

by

Megan Kathleen McLaurin

January 2011 
(C) 2011 Megan Kathleen McLaurin 


\begin{abstract}
Index-insurance is becoming increasingly popular because of its ability to provide low-cost, relatively easy to implement agricultural insurance for vegetation types whose productivity has been notoriously difficult to measure and to farmers in less-developed nations where traditional crop insurance schemes are not reasonable to implement. This study examines if the remotely sensed Normalized Difference Vegetation Index (NDVI) can be an effective basis for index-based crop insurance over a diverse set of locations. To do this I compare AVHRR-NDVI values to cumulative precipitation, extreme heat, and crop yields for sixty locations across the United States for the years 1982-2003. I use simple OLS quadratic equations to explore these relationships. My findings suggest that the relationship between NDVI, precipitation, extreme heat, and crop yields is highly variable and dependent on location-specific characteristics. Without site-specific calibration, NDVI should not be widely applied to index-based insurance product design. However, NDVI may still be a useful tool in insurance design under certain circumstances. Further research is needed to better characterize the factors affecting NDVI values and their relationships to crop yield.
\end{abstract}




\section{BIOGRAPHICAL SKETCH}

Megan McLaurin was born in coastal Wilmington, North Carolina to parents who were both university professors and to two loving sisters. From the beginning she had a great love of the outdoors, the arts, and the pursuit of academic knowledge.

Megan was eager to embark on her college education and began attending Barnard College in August 2003. While at Barnard she majored in Environmental Policy and minored in Women's Studies, graduating with a Bachelor's of Arts in May of 2007. Following graduation, Megan continued into a research assistantship position at Columbia University's International Institute for Climate and Society, where she worked for two years. During this time she nourished her passion for environmental and agricultural systems, becoming particularly fascinated by the development of economic tools allowing for their successful management.

Megan began her M.S. program in the field of Applied Economics and Management at Cornell University in August 2009. At Cornell, she has sought to deepen her knowledge of economic and financial structures as they relate to environmental and agricultural management. Upon completion of her Master of Science degree, Megan intends to pursue an education in environmental law, while retaining a focus on those issues most pertinent to agriculture. 


\section{ACKNOWLEDGMENTS}

I would like to thank my advisor Calum Turvey for the great deal of guidance, support, and encouragement he has given in the past 15 months, to help me make this a successful research endeavor. Additionally, I must thank him for the generous funding he made available to support my time and research at Cornell. I would also like to thank my special committee member Peter Hobbs, for his agronomic expertise and advise.

I am also thankful for the Knight Institute for Writing in the Disciplines and Chris Barrett, who made available a teaching assistantship, which provided me with an additional semester of funding as well as the invaluable experience of teaching Cornell undergraduates writing and logic skills as they apply to contemporary economic issues. I also owe much appreciation to Keith Hjortshoj at the Knight Institute for his insights into the writing process. For encouraging me to embark on my education in applied economics, I am also appreciative of the mentoring of Daniel Osgood, at IRI, Columbia University. 


\section{TABLE OF CONTENTS}

Biographical Sketch iii

Acknowledgements iv

Table of Contents $\quad \mathrm{v}$

List of Figures vi vi

List of Tables vii

1. Introduction 1

2. NDVI as a Measure of Crop Indemnity, Prior Studies and Current $\begin{array}{ll}\text { Projects } & 5\end{array}$

3. Data and Methodology 15

4. Results and Discussion 21

5. Conclusion and Suggestions for Further Research 41

$\begin{array}{ll}\text { Appendix A } & 46\end{array}$

$\begin{array}{lr}\text { Appendix B } & 104\end{array}$ 


\section{LIST OF FIGURES}

3.1 Location of Study Sites 17

4.1 Corn Yields and NDVI for Saluda, SC 30

4.2 Soy Yields and NDVI for Saluda, SC 30

4.3 Corn Yields and NDVI for Windsor, IL 34

4.4 Soy Yields and NDVI for Windsor, IL 34

4.5 Corn Yields for Saluda, SC and NDVI-based insurance payouts 38

4.6 Corn Yields for Windsor, IL and NDVI-based insurance payouts 38

4.7 Soy Yields for Saluda, SC and NDVI-based insurance payouts $\quad 39$

4.8 Soy Yields for Windsor, IL and NDVI-based insurance payouts $\quad 40$ 


\section{LIST OF TABLES}

4.1 Regression of NDVI on meteorological variables for Saluda, SC

4.2 Regression of NDVI on meteorological variables for Winsor, IL

4.3 Regression of NDVI on meteorological variables for Angelica, NY

4.4 Regression of corn yields on NDVI for Saluda, SC

4.5 Regression of soy yields on NDVI for Saluda, SC

4.6 Regression of soy yields on NDVI for Windsor, IL

4.7 Regression of soy yields on NDVI for Windsor, IL 
CHAPTER 1

Introduction

Index-based agricultural insurance is gaining increasing popularity due to its ease of implementation, its safeguards against the moral hazard and adverse selection problems frequently found in traditional agricultural indemnity insurance, and its resulting affordability (Hellmuth et al. 2009, Chantarat 2009, Barnett 2005). In the developing world, index-based insurance is seen as a method of providing risk protection to communities previously thought to be uninsurable (Skees 2008, Barrett et al. 2007). In developed countries, it provides an inexpensive method of hedging against weather risk in pasture, rangeland, and large monocultures (USDA 2009, Rowley et al. 2007,).

To date, most index-based insurance products are dependent on meteorological data collected via weather stations. This makes these products dependent on a strong meteorological information infrastructure, which often is non-existent in many of the developing countries that are the target clientele for this innovation in agricultural insurance. Increasingly, satellite information, in particular the Normalized Difference Vegetation Index (NDVI) is being promoted as a method of overcoming these weather information deficiencies (Ceccato et al. 2009, Hazell et al. 2010). As a result, programs employing NDVI data in index-based agricultural insurance currently exist in Canada, the United States, Spain, Kenya, and India (Diaz-Caneja et al. 2009).

NDVI is a satellite product that measures the vigor and greenness of vegetation on the earth's surface (Tucker 1985). The idea behind using NDVI 
in index-based insurance is that by measuring greenness, NDVI provides a description of vegetative health at any given time, and in turn, should be closely correlated with crop yields and primary production. NDVI values are thought to reflect the many variables affecting crop growth and distress, leading to a more comprehensive expression of crop health than an index based on one or two weather variables alone (Atwood et al. 2005, Makaudze and Miranda 2009, Hazell et al. 2010) Furthermore, NDVI data is available in an uninterrupted dataset of 21 years for the entire globe.

Most of the literature on NDVI is centered in the remote sensing community, and research on its ability to serve as a proxy for yield data or as a measure of primary productivity has arrived at mixed conclusions (du Plessis 1999, Makaudze and Miranda 2009). However, for insurance purposes, it is not necessary for NDVI to have strong enough correlations with yield and primary productivity to reflect slight fluctuations in crop growth. Insurance fundamentally deals with anomalous losses. Some year-to-year variation in crop yield is simply part of the business of agriculture; crop insurance is in place to protect the farmer against the event of extreme crop loss. Indexbased insurance, in particular, functions as a hedging product intended to protect a policy owner from environmental shocks (Syroka 2006). Thus, the ability of NDVI to capture years of extreme crop loss is of the greatest interest. Yet, there has been little research linking NDVI to crop indemnities, particularly in the economics literature.

This study contributes to the literature exploring the relationship between NDVI, meteorological variables and crop productivity by examining the relationship between NDVI and indemnity events. Specifically, it seeks to determine if NDVI can be applied generally as a substitute for meteorological 
and yield data in the development of index-based agricultural insurance products. For NDVI to be an adequate base for generalized index-based agricultural insurance products, it needs to be a reliable indicator of acute crop loss across a variety of locations representing an assortment of growing practices and diverse climate regimes. With its abundance of meteorological data and its diverse geography and farming practices, the United States provides an ideal setting for exploring this question. I use meteorological data from 59 weather stations across the United States to explore the relationship between NDVI and weather variables. I then use a subsample of 25 locations to compare NDVI with yield data directly. My findings suggest that NDVI cannot be applied generally as a reliable indicator of crop loss, though it may work under specific conditions and in select locations, or when used in conjunction with in situ data.

The overall objective of this paper is to investigate the potential of NDVI for use in index-based crop insurance products. Specific objectives include investigating the relationship between NDVI, precipitation and extreme heat for locations across the United Sates, and assessing the relationship between NDVI and crop yields for a wide variety of locations possessing diverse characteristics. To do this I first use a simple robust OLS quadratic function to regress NDVI on precipitation and extreme heat measures. I then apply a robust OLS quadratic equation to estimate the relationship between yields and NDVI. To look at the relationship between NDVI and crop indemnity more directly, I also examine the applicability of an NDVI-based insurance contract across several locations for the soy and corn crop.

The remainder of this thesis first describes the literature on NDVI as it relates to meteorological variables and predictions of crop yields and some of 
the NDVI-based insurance products currently on the market. Then I present the data and methodology used for this study, followed by the results of my analysis. I end with a discussion of the results and the insights they provide into applications of NDVI in index-based insurance products. 


\section{CHAPTER 2}

NDVI as a Measure of Crop Indemnity, Prior Studies and Current Projects

\section{Relationships between NDVI and meteorological variables}

Several studies exploring the relationship between NDVI and climate variables carried out in the United States' Great Plains found varying results on the influence of these variables on NDVI (Di et al. 1994, Tieszen et al. 1997, Wang et al. 2001 and 2003, Tan 2007, Yang et al. 1998). In a study using precipitation data from 410 weather stations and temperature data constructed from 17 weather stations across the state of Kansas, Wang et al. found precipitation to be the dominant climate regime influencing both temporal (2001) and spatial (2003) NDVI variation. However, other research suggests that temperature has greater influence on NDVI variation in much of the Great Plains (Tan 2007, Yang et al. 1998, Zhou et al. 2001).

Differences in studies likely result from the size of the study area and climate regimes covered, as the climate variable most affecting NDVI appears to vary depending on vegetation type and frequency of rainfall events. Yang et al. (1998) collected climate data from 72 automated weather stations across the northern and central Great Plains, and found summer and spring precipitation to be the dominant climate control on grassland NDVI in the southwest region of the study site, while NDVI was strongly influenced by temperature and precipitation in the northern region of the study site. This was accredited to the predominance of plants with C3 photosynthetic pathways in the north and those with $\mathrm{C} 4$ photosynthetic pathways in the south. 
Growing degree days and soil temperature were also found to be closely correlated with NDVI in Nebraska (Yang et al. 1998).

In comparing data for grassland, cropland, and forest, NDVI has the strongest correlation with precipitation in the grassland vegetation regime. NDVI correlation is also stronger in instances where there are distinct precipitation events, meaning a wet year following a dry season or a series of dry seasons. Precipitation data from the preceding growing season also seems to play an important role in the NDVI for the current growing season (Wang et al. 2001).

In Africa, meaningful direct relationships have been found between NDVI, rainfall and vegetation cover in many studies carried out in the Sahel Zone (Tucker et al. 1985; Hielkema et al., 1986, Malo and Nicholson 1990), Botswana (Prince and Tucker 1986), East Africa (Boutton and Tieszen 1983, Davenport and Nicholson 1993) and Tunisia (Kennedy 1989). However, findings for these locations were still highly variable. Nonetheless, it is often concluded from this body of work that NDVI and precipitation have a strong linear (Malo and Nicholson 1990) or log-linear (Davenport and Nicholoson 1993) relationship, when monthly and annual precipitation is within a range that makes rainfall a limiting factor on vegetation growth. This last condition, confines the strongest relationships between NDVI and precipitation to regions where annual rainfall is within a specific range, identified as between 300 and $900 \mathrm{~mm}$ in South Africa (Richard and Poccard 1998), between 500 and $700 \mathrm{~mm}$ in China (Li et al. 2002), and less than $700 \mathrm{~mm}$ with an average rainfall/PET ratio of 0.5 to 2 in Spain (Udelhoven et al. 2009).

Despite these conclusions, the highly variable relationships between rainfall, temperature and NDVI exhibited in these collective studies warrant 
caution in relying on NDVI in applied work. Differences in soil and vegetation type, time of year, and span of time contribute to some of this variation. Farrar et al. (1994) found NDVI to only reflect changes in precipitation for specific soil types. Furthermore, some variability is likely the result of complex radiative interactions between the atmosphere, sensor view angle, and solar zenith angle, which all effect NDVI value accuracy and are difficult to account for in standard adjustments (du Plessis 1999). Numerous scientists in the remote sensing community have warned of NDVI's limitations (Ceccato et al. 2009, Lillesand and Kiefer 1994). However, these limitations are rarely considered in most NDVI research and NDVI data applications.

Regions where the precipitation regime is not of a range that characterizes it as a limiting factor to plant growth, soil moisture appears to become the dominant climate variable affecting NDVI values. Farrar et al. (1994) found soil moisture of the concurrent month to have a strong effect on NDVI values in Botswana, once rainfall was below a threshold of $500 \mathrm{~mm}$. In a study of NDVI and rainfall anomalies in Spain, Udelhoven et al. (2009) found water deficit, not rainfall amount, to be the predominant factor determining NDVI anomalies. As a result of this, NDVI was poorly correlated with rainfall in arid and humid regions, where soil water content is quickly depleted after a rainfall due to evapotranspiration in the former, and frequent rainfall and low evapotranspiration rates create a buffer of soil moisture in the latter. This corresponds to Yang et al.'s (1998) finding that NDVI and evapotranspiration are negatively correlated.

Collectively, the work on relationships between NDVI, precipitation and temperature is characterized by highly variable relationships. This implicates the strong influence of other factors, such as soil and vegetation type, 
geographic region, climate zone, and radiometric disturbances of NDVI measurement in the ability of NDVI to reflect changes in precipitation and temperature.

\section{Relationships between NDVI and agricultural productivity}

Much like the relationship between NDVI and meteorological variables, the relationship between NDVI and agricultural productivity appears to be highly variable depending on a wide variety of additional site-specific characteristics. Box et al. (1989) found the relationship between NDVI and primary productivity to be consistent across the globe, with NDVI possessing a predictive accuracy comparable to climate-based models of annual net primary productivity. However, their study also found NDVI values to be unreliable in areas of complex terrain, such as those characterized by coastlines, high mountains, or dry regions using irrigation.

The relationship between NDVI and forage production has also been established in a number of studies, both in the U.S. and abroad (Paruelo and Lauenroth 1995, Fuller 1998). In their study of grasslands throughout Montana, Thoma et al. (2002) found NDVI to explain $63 \%$ of the statistical variation in live biomass. This relationship was consistent for all of six of their sample locations, which spanned north and south Montana. Similarly, Kennedy (1989) found strong correlations ( $r=0.85$ and $r=0.90)$ between NDVI aboveground green biomass in grazing lands in Tunisia. In what is perhaps a more comprehensive study, du Plessis et al. (1999) found NDVI to predict green aboveground biomass in Etosha National Park, Namibia with a coefficient of determination of 0.5166 , a statistical relationship they describe 
as "poor," while many papers stating similar values describe the relationship as "meaningful" or "strong."

Establishing a relationship between crop vigor during the growing season (NDVI) and final crop production for field crops is not as simple, as final crop yields depends on a range of factors including nutrients, solar radiation, and water stress during critical stages of plant growth which may not be reflected in NDVI values (Ceccato et al. 2009). Furthermore, NDVI appears to perform most accurately over large areas of grassland (Yang et al. 1998, du Plessis et al. 1999, Thoma et al. 2002), which differ from the multi-layered nature of vegetation present in cropland. Farmland also often contains a multitude of vegetation types, including several crops, often rotated, each with different planting and harvesting times. These factors all add to the complexity involved in measuring crop health using satellite data.

In Swaziland, Mkhabela et al. (2004) discovered that NDVI could be used effectively to forecast maize yield in three of the country's four agroecological regions. Lewis et al. (1998) found NDVI to be a good indicator of maize production in a study using annual maize production statistics for 36 agricultural districts in Kenya. Nonetheless, just as variability characterizes the relationships between NDVI, temperature and rainfall, it is a defining characteristic in the relationships reported between NDVI, vegetation, and crop yield in the literature. This points to a need to clarify the limitations of NDVI in order to best understand the potential for future applications.

\section{Investigating applicability of NDVI in index-based insurance}

A very few studies exist directly exploring NDVI's potential for use in insurance products. Majority of those few focus on NDVI for use in rangeland 
and pasture insurance, due to the particular difficultly in measuring rangeland and pasture productivity, since it does not possess the equivalent of yield data, the common measurement of loss used in crop insurance. In the United States, Rowley et al. (2007) compared rancher perception of low production years to NDVI data for several counties in Kansas and Oklahoma. Their study found weak statistical relationships between individual range productivity and NDVI values $\left(R^{2}=0.15\right)$. However, when this data was aggregated to the county level, the relationship between NDVI values and rancher perception of productivity improved substantially $\left(R^{2}=0.65\right)$. This discrepancy between ranch and county results illustrates the fundamental issue of basis risk that is continuously acknowledged as one of the main limitations of index-based insurance products (Barnett 2004).

Rowley et al. (2007) also found NDVI failed to capture mid-season low rainfall events flanked by periods of high rainfall. While ranchers classified such years as years of poor range productivity due the critical timing of the low-rainfall event, NDVI was unable to mark this effect on range conditions. Atwood et al. (2005) suggest a dual trigger mechanism on insurance products to overcome some of these problems. Their investigation into using NDVI suggests that it is a highly competent tool for rangeland insurance purposes, as long as crop rotation and technology trending are not factors within the rangeland and the annual range production is not dependent on end of season conditions.

Also working in rangeland conditions, Chantarat (2009) used NDVI data to design an index-based livestock insurance contract for pastoralists in Northern Kenya. With this data, she was able to establish a predictive 
relationship for livestock mortality, using vegetative conditions derived from NDVI values.

Makaudze and Miranda (2009) assessed the possibility of using NDVI for crop insurance for corn and cotton crops in Zimbabwe. While the authors conclude that NDVI holds great potential for index-based insurance, their results suggest the relationship between NDVI and crop yields to be highly variable between crops and districts. They discover a correlation between NDVI during critical growth stages of the crops and yields ranging from 0.42 to 0.70 , depending on crop and district. The timing of this critical growth stage also varies depending on district location, thus requiring calibration based on farmer input on a district-by-district basis.

\section{Existing index-based agricultural insurance projects using NDVI data}

NDVI-based insurance programs currently exist in the USA, Canada, India, Kenya, Ethiopia and Spain. The USDA's Risk Management Agency (RMA) runs a pilot vegetation index insurance program for pasture, rangeland and forage. The program has been available since 2007 in select states, and was expanded in 2009 to include additional states. States were chosen such that the index would be tested in various climate, soil and weather conditions via six US regions: the warm and humid Southeast, the cool and humid Northeast, the Northern Great Plains, the Southern Great Plains, the semi-arid Southwest, and the intermountain region of the Northwest. Farmers select one or more 3-month time periods that represent the high-risk time period for their pasture, rangeland, and forage management practices. NDVI data used to evaluate loss is in the form of a $4.8 \times 4.8$ mile grid, and losses are evaluated according to the grid, not losses experienced at individual properties. Losses 
are calculated as the difference between the expected normal NDVI value and the actual NDVI value experienced for the interval the farmer chose to insure. A payment is received when this number falls below a certain trigger grid index (USDA 2009).

NDVI-based insurance in Canada was launched in 2001 by Agriculture Financial Services Corporation (AFSC). The insurance is limited to area where pasture is the predominant landcover and is intended to cover hay production. NDVI data is calculated for each township in the area and is scaled to reflect native pasture production. Areas of irrigated cropland and bush are removed, as they can significantly influence the program outcome. A pasture vegetation index (PVI) is developed for each square kilometer of the township. Pasture production data collected by AFSC during a past cage clipping system operation was used for correlation comparisons from 19911999. Precipitation data measured at Environment Canada weather stations was also compared to NDVI data, and client meetings were conducted where farmers identified their two best and two worst pasture production years in the last fifteen year period. Historical PVI values seemed to identify production shortfalls resulting from cool early season temperatures and drought. In addition, the anecdotal production perception of farmers surveyed corresponded to geographical differences between township PVI values. NDVI correlations with precipitation data and collected production data were not considered good, with an $r=0.65$.

AFSC augmented NDVI data by collecting precipitation and pasture growth data over the growing season at test stations throughout the insured pasture area. This substantially improved correlations between the developed PVI index, precipitation and pasture production (Diaz-Caneja et al. 2008). 
Thus, the developed insurance index takes into account production data alongside NDVI data. A loss payment is triggered when the current year's PVI falls below 90-85 percent of the average PVI from previous years (the percentage depends on if the farmer has full-season or split-season coverage) (AFSC 2009).

Spain's NDVI-based insurance was designed to protect farmers from droughts affecting pasture areas. It has also been available since 2001, but unlike Canada's insurance scheme, Spain's insurance index is solely based on NDVI data; it is not verified by any additional measurement of yields. The index is constructed using historical NDVI values for the given area to create a curve of expected NDVI values. When actual NDVI observations in a given year fall below the average curve an indemnity is defined. NDVI values are defined for a ten-day period. The maximum value for each ten-day period is used to reduce the effect of clouds; this is called the Maximum Value Composite Index ( $\mathrm{MVCl})$. The deductible is the historic average $\mathrm{MVCl}$ for each area minus 1.25 standard deviations from the average MVCI (DiazCaneja et al. 2008).

The Agricultural Insurance Company of India (AIC) uses NDVI data combined with temperature parameters for their wheat insurance index. NDVI and temperature data from the peak crop growth stage is used to determine if there is to be a payout (AIC 2009). AIC is also in the process of carrying out research intended to aid in developing an NDVI-based index insurance product for tea production.

A pilot index insurance project for several Millenium Villages locations uses NDVI as its basis. The project used NDVI data with an $8 \mathrm{~km}$ resolution, aggregating data to an average area of $100 \mathrm{~km} \times 100 \mathrm{~km}$. The index targeted 
the time between flowering and harvest in local cropping calendars, since this was found to have the strongest relationship between local rainfall and historical yields. NDVI was found to be sufficient to underpin contracts in regions of Senegal, Mali, Ethiopia, northern Nigeria and northeastern Kenya because of their semi-arid climates. NDVI was used in combination with rainfall for the wetter, less variable climates of Uganda, Rwanda, Tanzania, Malawi, and western Kenya. Research for these projects found that when native vegetation shows signs of stress at the $100 \mathrm{~km} \times 100 \mathrm{~km}$ scale, crop yields are typically greatly reduced (Hellmuth, M.E. et al. 2009).

The combination of a growing number of projects implementing NDVIbased index insurance products and the highly ambiguous results presented in the literature regarding the relationship between NDVI, meteorological variables, agricultural productivity, and crop yields presents a great need for better understanding of the reliability of NDVI in assessing crop indemnities. Current research suggests the ability of NDVI to reflect variations in rainfall and agricultural productivity is highly dependent on location of the study and timing of the research. 


\section{CHAPTER 3}

Data and Methodology

\section{The Normalized Vegetation Index (NDVI)}

The Normalized Difference Vegetation Index (NDVI) is a satellite product that measures the vigor and greenness of vegetation on the earth's surface. It is calculated as the ratio of visible spectral wavebands to nearinfrared spectral wavebands. Healthy, green vegetation has a high presence of chlorophyll pigment, which causes low reflectance in visible wavebands and high reflectance in near-infrared wavebands. The reverse is true in vegetation under stress.

NDVI is a unit-less index, with values ranging from -1 to 1 . Healthy vegetation has the highest positive values, while bare soil, water, snow, ice, or clouds have NDVI values of zero or that are slightly negative. Vegetation under stress or with a small leaf area has lower positive NDVI values. Typically the NDVI values from healthy vegetation will increase as plant cover increases at the beginning of the growing season, reach a peak sometime during the middle of the growing season, and will then decrease as the season comes to its end (Mkhabela et al. 2005).

For this study, I will use the AVHRR-NDVI dataset provided by the University of Maryland. The AVHRR-NDVI dataset is desirable because it is a global gridded dataset, with an $8 \mathrm{~km} \times 8 \mathrm{~km}$ spatial resolution. Additionally, the AVHRR-NDVI dataset has been recommended for use in index-based insurance products above alternative satellite options (Atwood et al. 2005). The dataset begins in 1981 and goes through 2003, with bimonthly NDVI 
estimates. The NDVI value for each bimonthly period represents the maximum NDVI for that period. This is the most effective method of reducing errors resulting from cloud cover and similar distortions. I use NDVI data for the grid point associated with each of the 60 meteorological stations. Data was collected for an estimated growing season, beginning May 1 and ending August 15 and containing seven bimonthly periods.

\section{Meteorological and Crop Yield Data}

Meteorological data was obtained from NOAA, via the internet-based weather risk evaluation tool WeatherWizard (Norton and Turvey 2007). I collected cumulative precipitation, average mean daily temperature, and cumulative growing degree days for each bimonthly period corresponding to the NDVI data. Growing degree days were calculated using an 80 degrees Fahrenheit standard in order to capture extreme heat.

County-level crop yield data was selected for a sub-sample of 29 stations for 1982-2003. This included yearly yield data for hay, corn, soybeans and wheat depending on the crops grown in the selected counties. This data was obtained from the USDA's NASS.

\section{Site Locations}

All the sites selected are located in heavily farmed areas known to use little or no irrigation. Locations of meteorological stations are shown in Figure 3.1. Circles mark the 60 meteorological stations. Those stations where county level yield data was also collected have an X to their left. 


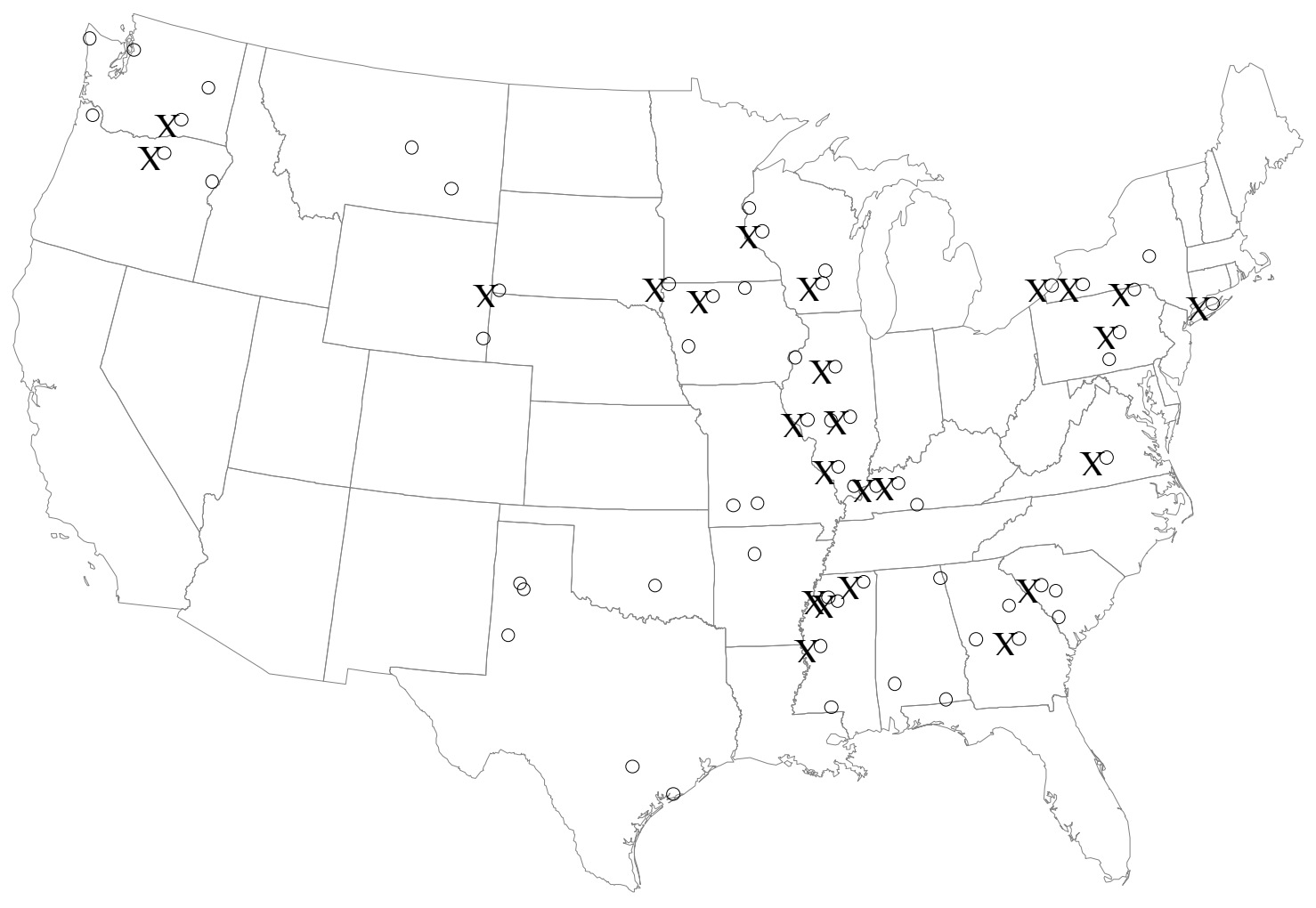

Figure 3.1. Location of study sites. Circles represent location of meteorological stations. X's represent locations for which county level yield data was also collected.

The sites span variety of climate regions within the U.S. Five are located in coastal areas, and one on Lake Eerie. The locations also represent a range of elevations, from sea level to $1,485 \mathrm{~m}$. 


\section{NDVI and Meteorological Variables}

I first compared NDVI to cumulative precipitation and growing degree days using the quadratic equation below

\section{(Equation 3.1)}

$$
N D V I_{y p}=\mu+\alpha_{y}+\gamma_{p}+\beta_{1} c p_{y p}+\beta_{2 g} g d d_{y p}+\beta_{3} c p_{y p}{ }^{2}+\beta_{4} g d d_{y p}{ }^{2}+\beta_{5} c p g d d_{y p}+\varepsilon_{y p}
$$

where $N D V I$ is the bi-monthly values for $N D V I$ at a given station, $c p$ is cumulative precipitation for the same bi-monthly period, $g d d$ is the cumulative growing degree days for the period, and $\alpha$ is the constant term. The quadratic function was used in order to capture interaction effects between the variables, as precipitation and heat, as well as the joint effect of the two, are important in identifying instances of severe crop distress and loss. Using the growing degree day standard of $80^{\circ} \mathrm{F}$, allows the $g d d$ variable to capture instances of extreme heat. Thus, this equation should not only be reflecting the vegetation stress caused by a lack of rainfall or extreme heat, but also the combined effect of rainfall and extreme heat events. I used this equation to carry out regressions for each of the 59 locations.

I selected this model after exploring both linear and log-linear models, since prior studies have suggested these to describe the relationship between NDVI and precipitation. However, these studies were describing relationships within a specific climate regime, and where grassland was the predominant groundcover. For the variety of locations we are assessing, the quadratic model presents a more comprehensive method of exploring the relationship between NDVI, cumulative precipitation, and growing degree days. Initial regressions included mean temperature in addition to these variables, but 
mean temperature was not a significant variable in any of the locations, nor did it appear to contribute additional information relating to temperature, as this study is most interested in cases of extreme heat, best captured using the growing degree measure.

\section{NDVI and Crop Yields}

Since the relationship of most importance in an index-based insurance product is between the data used in the index itself and crop yields, I selected a subsample of 25 stations to use in comparing crop yields and NDVI directly. Again, I used a quadratic equation in order to capture interaction effects between precipitation and heat, this time using crop yield as the dependent variable, and adding NDVI into the equation as an independent variable. The model used is presented below

\section{(Equation 3.2)}

yield $=\alpha+\beta_{1} N D V I+\beta_{2} c p+\beta_{3} g d d+\beta_{4} N D V I^{2}+\beta_{5} c p^{2}+\beta_{6} g d d^{2}+\beta_{7} c p N D V I+\beta_{8} g d d N D V I+\beta_{9} c p g d d$

where yield is county-level yield data for years 1982-2003; NDVI is the maximum NDVI value during the growing season; $c p$ is cumulative precipitation for the growing season; gdd is total growing degree days during the growing season (again measured using the $80^{\circ} \mathrm{F}$ standard), and $\alpha$ is the constant term. As previous studies have suggested both integrated NDVI and maximum NDVI as the best predictors of crop yield, I also performed a regression analysis using integrated NDVI for the growing season as the NDVI variable. Results for the two measures were not significantly different, and all results presented here are for maximum seasonal NDVI values. 
The analysis was carried out for each of the 25 locations using countylevel corn yields. I also fit the model using hay, wheat, and soybean yields for those stations in which these crops were relevant and the data was available. In the case of wheat, this was all stations except Algona, IA and Montrose, PA. For hay, this excluded stations located in Missouri. And for soybeans, this excluded Montrose, PA, Heppner, OR, Eltopia, WA, Ardmore, SD and stations located in New York. 


\section{CHAPTER 4}

\section{Results and Discussion}

\section{NDVI and Meteorological Variables}

\section{General Trends}

Results of the regressions of NDVI on meteorological variables were mixed across stations, without apparent trends in which meteorological variables are significant in determining NDVI, or even common signs on coefficients for the same variables. Cumulative precipitation was a significant variable in determining NDVI for 7 of the 59 stations. Coefficients for $c p$ were negative in 39 of the 59 stations. $c p^{2}$ had a negative coefficient in 22 of the locations, and was only significant for 8 of the 59 stations. Similarly, gdd was a significant factor in determining NDVI for 4 stations, and had negative coefficients in 27 of the locations. $g d d^{2}$ was only significant in 5 stations, and its coefficient was negative for 28 locations. The interaction term cpgdd was only significant for 6 stations, and had a negative coefficient in 22 locations.

The coefficient of determination for the model when applied to the 59 stations ranged from 0.2435 to 1.0 , with 49 percent of stations having $R^{2}$ values greater than 0.6. Those stations with higher $R^{2}$ values did not show more consistency in coefficient signs across variables, and had mixed results in which variables were significant in determining NDVI. It is clear from this assessment that $R^{2}$ as a matter of course is not meaningful without also having consistency in parameters. However, across stations, the F-tests agree that the model has explanatory power. Appendix A includes complete results from the regression analysis. In the regression analysis of several 
stations, $G D D, G D D^{2}$, and in some cases, $C P G D D$ was dropped from the model. These are instances where there were no days where average temperatures rose above $80^{\circ} \mathrm{F}$.

\section{Case Studies: Saluda, SC, Windsor, IL and Angelica, NY}

Because of the large number of regressions performed in this study, 176 in total, only a few case studies are presented and discussed in detail here. Complete results are included in the appendices. I attempted to select case studies representative of the wide variety of results observed in this analysis.

Results for the regression of NDVI on meteorological variables at three of the locations are presented in Tables 4.1, 4.2, and 4.3. Of these three examples, Saluda had the lowest $R^{2}$ value and none of the meteorological variables are statistically significant in determining NDVI for this location. Nonetheless, the F-test for this regression implies the results do have legitimate explanatory power. The lack of statistical significance of the coefficients on meteorological variables is not necessarily alarming, as the true marginal effects of cumulative precipitation and extreme heat on NDVI are revealed by taking the partial derivatives of the regression with respect to $c p$ and gdd. 
Table 4.1. Results for regression of NDVI on meteorological variables for Saluda, SC

\begin{tabular}{|c|cccccc|}
\hline Variable & $\beta$ & Std. err. & $\mathrm{t}$ & P-value & \multicolumn{2}{c|}{$95 \%$ Confidence Int. } \\
\hline$C P$ & .0031002 & .000611 & 0.51 & 0.613 & -.0089987 & .0151991 \\
$G D D$ & .0089049 & .2681629 & 0.03 & 0.974 & -.5219943 & .5398042 \\
$C P^{2}$ & -.0008605 & .0008164 & -0.98 & 0.329 & -.002598 & .000877 \\
$G D D^{2}$ & -.0009544 & .0007525 & -0.01 & 0.991 & -.1700828 & .1681739 \\
$C P G D D$ & .0050411 & .0066899 & 0.15 & 0.884 & -.0633812 & .0734636 \\
\hline$R^{2}$ & & & & & & 0.3270 \\
\hline
\end{tabular}

The weather response function for NDVI at Saluda, SC is shown in Equation 4.1.

\section{(Equation 4.1)}

$$
N D V I=0.73797+0.00310 c p+0.00890 g d d-0.00861 c p^{2}-0.00095 g d d^{2}+0.00504 c p g d d
$$

Solving for $\frac{\partial N D V I}{\partial c p}=0$ and $\frac{\partial N D V I}{\partial g d d}=0$ simultaneously results in $g d d^{*}=-5.36405$ and $c p^{*}=-2.78197$. While the negative optimum for $g d d$ is expected, as it represents a sensitivity of NDVI to extreme heat, the negative optimum for $c p$ does not appear reasonable, as we would expect higher levels of rainfall to lead to increased levels of vegetation greenness.

In the case of Windsor, IL (see Table 4.2), I also did not find a significant relationship between NDVI and each meteorological variable using this model. Signs on coefficients differed from those in Saluda, SC for every variable, with the exception of $c p$. However, the coefficient of determination for this station is stronger $\left(R^{2}=0.8429\right)$, and the $F$-test also shows the model to possess significant explanatory power. 
Table 4.2. Results for regression of NDVI on meteorological variables for Windsor, IL

\begin{tabular}{|c|cccccc|}
\hline Variable & $\beta$ & Std. err. & $\mathrm{t}$ & P-value & \multicolumn{2}{c|}{$95 \%$ Confidence Int. } \\
\hline$C P$ & .0011286 & .015059 & 0.07 & 0.940 & -.0286896 & .030947 \\
$G D D$ & -.0001142 & .0010117 & -0.11 & 0.910 & -.0021175 & .0018891 \\
$C P^{2}$ & -.0004187 & .0020536 & -0.20 & 0.839 & -.0044849 & .0036476 \\
$G D D^{2}$ & $3.55 \mathrm{e}-06$ & $7.41 \mathrm{e}-06$ & 0.48 & 0.633 & -.0000111 & .0000182 \\
$C P G D D$ & -.0000677 & .000192 & -0.35 & 0.725 & -.0004479 & .0003125 \\
\hline$R^{2}$ & & & & & & \\
\hline
\end{tabular}

The weather response function for Windsor, IL NDVI is given by (Equation 4.2)

$N D V I=0.40276+0.00113 c p-0.00011 g d d-0.00042 c p^{2}+3.55 e-06 g d d^{2}-0.00007 c p g d d$

Again, by setting partial derivatives with respect to $c p$ and $g d d$ equal to zero, we can solve for the optimum values of $c p$ and $g d d$. This results in $c p^{*}=-$ 0.00058 and $g d d^{*}=16.67096$. Still the optimum value of $c p$ is negative, while for this location, the optimum value of $g d d$ is positive. As the weather response function indicates a negative optimum value of cumulative precipitation to maximize NDVI, it appears that NDVI is not accurately reflecting the relationship between cumulative precipitation and vegetation condition.

In Angelica, NY, both cumulative precipitation and $c p^{2}$ are significant variables at the $20^{\text {th }}$ percentile. Cumulative precipitation has a negative coefficient, which does not reflect the positive correlation between rainfall and crop growth, yet $c p^{2}$ also has a positive coefficient, which is different from what 
we saw in the case of Saluda, SC and Windsor, IL. Again, we see a reasonable $R^{2}$ value (0.6782), falling in between the stronger and weaker values found in Windsor, IL and Saluda, SC, respectively. Here the F-test also shows the results to have explanatory power. Results for this analysis are displayed in Table 4.3.

Table 4.3. Results for regression of NDVI on meteorological variables for Angelica, NY

\begin{tabular}{|c|cccccc|}
\hline Variable & $\beta$ & Std. err. & $\mathrm{t}$ & P-value & \multicolumn{2}{c|}{$95 \%$ Confidence Int. } \\
\hline$C P$ & -.0214638 & .013715 & -1.56 & 0.120 & -.0486331 & .0057054 \\
$G D D$ & -.0059557 & .0149818 & -0.40 & 0.692 & -.0356344 & .023723 \\
$C P^{2}$ & .0034728 & .0021568 & 1.61 & 0.110 & -.0007999 & .0077455 \\
$G D D^{2}$ & .0002725 & .0008616 & 0.32 & 0.752 & -.0014344 & .0019794 \\
$C P G D D$ & .0051141 & .0042287 & 1.21 & 0.229 & -.003263 & .0134911 \\
\hline$R^{2}$ & & & & & & \\
\hline
\end{tabular}

Equation 4.3 depicts the weather response equation for Angelica, NY NDVI.

(Equation 4.3)

$N D V I=0.60129-0.02146 c p-0.00596 g d d+0.00347 c p^{2}+0.00273 g d d^{2}+0.00511 c p g d d$

By finding the particular derivatives with respect to $c p$ and $g d d$ and solving both simultaneously, setting each to 0 , we find optimum values of $c p^{*}=-1.679$ and gdd $^{*}=5.33743$. Again, the negative optimum cumulative precipitation value illustrates that NDVI is not effectively capturing the affect of precipitation on vegetation growth and health. 
These three locations represent the wide variety of relationships between NDVI, precipitation, and extreme heat across the many locations surveyed. Generally most instances where the model had a low coefficient of determination corresponded to locations in the southeast; however, this was not consistently the case and several of these instances were for stations located in other regions of the country. Differences in signs on coefficients for precipitation and extreme variables could not be explained simply by the station's regional location. Elevation and coastal location also did not account for inconsistencies between regression results across stations.

\section{NDVI and Crop Yields}

\section{General Trends}

Comparisons of crop yield data to NDVI values also produced ambiguous results. In estimating the quadratic model for corn yields, NDVI was a significant variable in three of the 25 locations. The estimated coefficients on NDVI were negative in $32 \%$ of locations. Coefficient of determination for the model ranged from 0.2043 to 0.862 . For a given location, signs on coefficients and which variables were statistically significant were not consistent across multiple crops. The two case studies presented below illustrate the wide variation in relationships between Yields, NDVI and meteorological variables across sites and crops. Complete regression results for all stations and crops are provided in Appendix B.

\section{Case Study: Saluda, SC}

Results for Saluda, SC, show the model to be fitting well, with a relatively high coefficient of determination when using corn and soy yield data 
(0.6606 and 0.7826 , respectively). For both crops, F-tests show the model to hold some significance.

Table 4.4. Results for Yields regressed on NDVI and meteorological variables, for corn at Saluda, SC

\begin{tabular}{|c|cccccc|}
\hline Variable & $\beta$ & Std. err. & $\mathrm{t}$ & P-value & \multicolumn{2}{c|}{$95 \%$ Confidence Int. } \\
\hline NDVI & -5162.91 & 13404.89 & -0.39 & 0.706 & -34122.41 & 23796.59 \\
$C P$ & 1.414855 & 29.85728 & 0.05 & 0.963 & -63.08787 & 65.91758 \\
$G D D$ & 14.25214 & 11.96689 & 1.19 & 0.255 & -11.60075 & 40.10502 \\
$N D V I^{2}$ & 3094.49 & 8757.491 & 0.35 & 0.729 & -15824.92 & 22013.9 \\
$C P^{2}$ & -.044294 & .2597625 & -0.17 & 0.867 & -.6054768 & .5168886 \\
$G D D^{2}$ & -.764791 & .3428671 & -2.23 & 0.044 & -1.50551 & -.0240713 \\
$C P N D V I$ & 4.016267 & 41.93387 & 0.10 & 0.925 & -86.57634 & 94.60888 \\
$G D D N D V I$ & Dropped & & & & & \\
$C P G D D$ & -.189214 & .9618443 & -0.20 & 0.847 & -2.267152 & 1.888724 \\
\hline$R^{2}$ & & & & & & \\
\hline
\end{tabular}

The only variable showing a significant relationship with corn yields in Saluda was $G D D^{2}$, which had the predicted negative coefficient. Soy yields, however, had a significant relationship with all but two of the variables $\left(C P^{2}\right.$ and $(P G D D$ ). See Tables 4.4 and 4.5. 
Table 4.5. Results for yields regressed on NDVI and meteorological variables, for soy at Saluda, SC.

\begin{tabular}{|c|cccccc|}
\hline Variable & $\beta$ & Std. err. & $\mathrm{t}$ & P-value & \multicolumn{2}{c|}{$95 \%$ Confidence Int. } \\
\hline$N D V I$ & -10648.65 & 2553.056 & -4.17 & 0.001 & -16211.28 & -5086.022 \\
$C P$ & -31.69274 & 10.51508 & -3.01 & 0.011 & -54.60312 & -8.782355 \\
$G D D$ & 4.832407 & 1.600502 & 3.02 & 0.011 & 1.345213 & 8.319602 \\
$N D V I^{2}$ & 6576.267 & 1568.007 & 4.19 & 0.001 & 3159.873 & 9992.661 \\
$C P^{2}$ & .0368131 & .0526719 & 0.70 & 0.498 & -.0779492 & .1515754 \\
$G D D^{2}$ & -.1174383 & .0449632 & -2.61 & 0.023 & -.2154047 & -.019472 \\
$C P N D V I$ & 40.50446 & 12.16288 & 3.33 & 0.006 & 14.00383 & 67.00509 \\
$G D D N D V I$ & Dropped & & & & & \\
$C P G D D$ & -.1642622 & .1284485 & -1.28 & 0.225 & -.4441275 & .115603 \\
\hline$R^{2}$ & & & & & & \\
\hline
\end{tabular}

The regression of corn yields on NDVI and meteorological variables yields the following equation

\section{(Equation 4.4)}

$$
\begin{aligned}
& \text { Yield }=2150.967-5162.91 N D V I+1.414855 c p+14.25214 g d d+3094.49 N D V I^{2}-0.044294 c p^{2} \\
& -0.764791 g d d^{2}+4.016267 c p N D V I-0.189214 c p g d d
\end{aligned}
$$

To measure the marginal effect of $N D V I, c p$, and gdd on corn yields I took the partial derivatives of this equation with respect to each of these three variables. I then evaluated these equations using the mean value for each variable to estimate the marginal effect of each on corn yield. This resulted in the following results, $\quad \frac{\partial \text { Yield }}{\partial N D V I}=-2739.48 \quad \frac{\partial \text { Yield }}{\partial c p}=3.59 \quad \frac{\partial \text { Yield }}{\partial g d d}=10.49$ 
where the marginal effects are the change in bushels of corn yielded for an increase in one unit of $N D V I, c p$, and $g d d$, respectively. The negative marginal effect of $N D V I$ on corn yields shows that in the case of Saluda, SC, increased NDVI values do not reflect increased yields for the corn crop. While the marginal effect of $c p$ on yields is positive, as would appear reasonable.

The yield function for soy yields in Saluda, SC, listed in Equation 4.5 was used to derive the marginal effects of $N D V I, c p$, and gdd on soybean yields, by taking the respective derivatives and evaluating them at the variables' means.

\section{(Equation 4.5)}

$$
\begin{aligned}
& \text { Yield }=4318.534-10648.65 N D V I-31.69274 c p+4.832407 g d d+6576.267 N D V I^{2} \\
& +.0368131 c p^{2}-.1174383 g d d^{2}+40.50446 c p N D V I-.1642622 c p g d d
\end{aligned}
$$

Again we see a negative marginal effect of $N D V I$ on yields, when using the soybean data, with $\frac{\partial Y i e l d}{\partial N D V I}=-5010.18$ as well as the expected positive relationship between $c p$ and soybean yields, with $\quad \frac{\partial Y i e l d}{\partial c p}=0.399$ This further suggests that NDVI is not accurately capturing changes in crop yields, nor is it accurately reflecting the effect of precipitation on crop yields.

Simple graphs showing the time series data for yields and NDVI for both corn and soy (Figures 4.1 and 4.2) do not show a clear relationship between the two datasets for either crop. For example, Figure 6.1 shows NDVI capturing the dip in corn yields in Saluda, SC in 1986, as well as increases in yields in 1987 and 1985. Yet NDVI fails to follow large drops in corn yields in 1983, 1993, and 2002. 


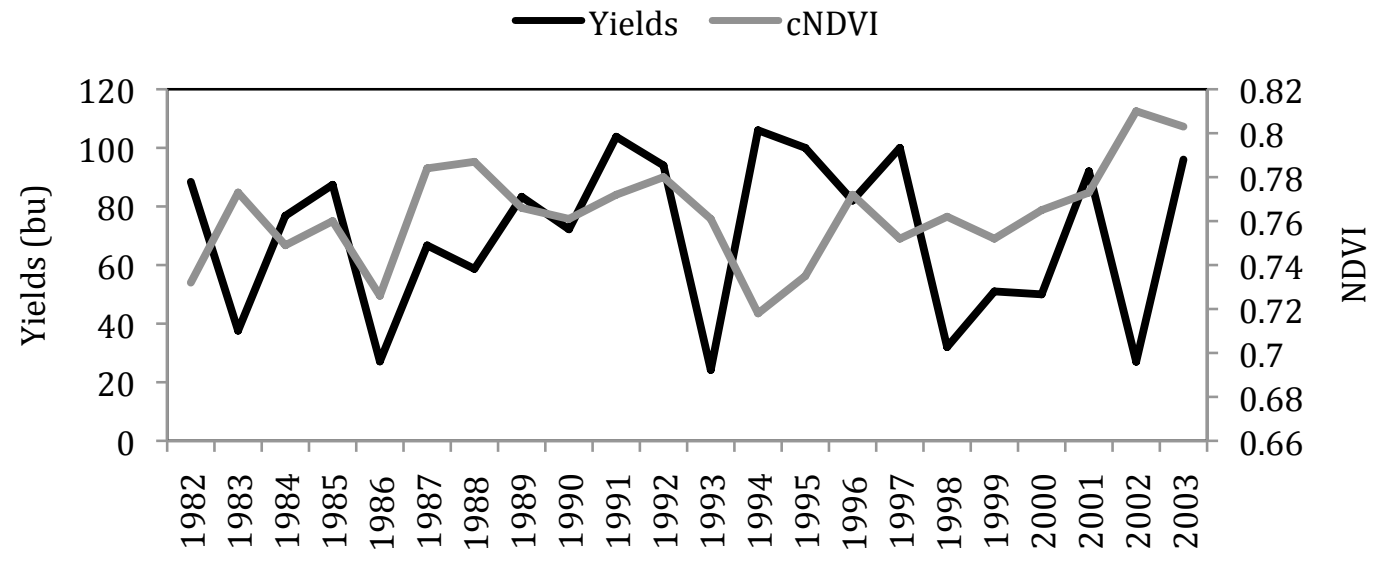

Year

Figure 4.1. Corn Yields and NDVI for Saluda, SC

Likewise, in the case of soy, shown in Figure 4.2, NDVI is at its lowest at one of the years with the greatest yields, 1994. NDVI also does not capture the strong dips in soy yields in 1993,1998, and 2001.

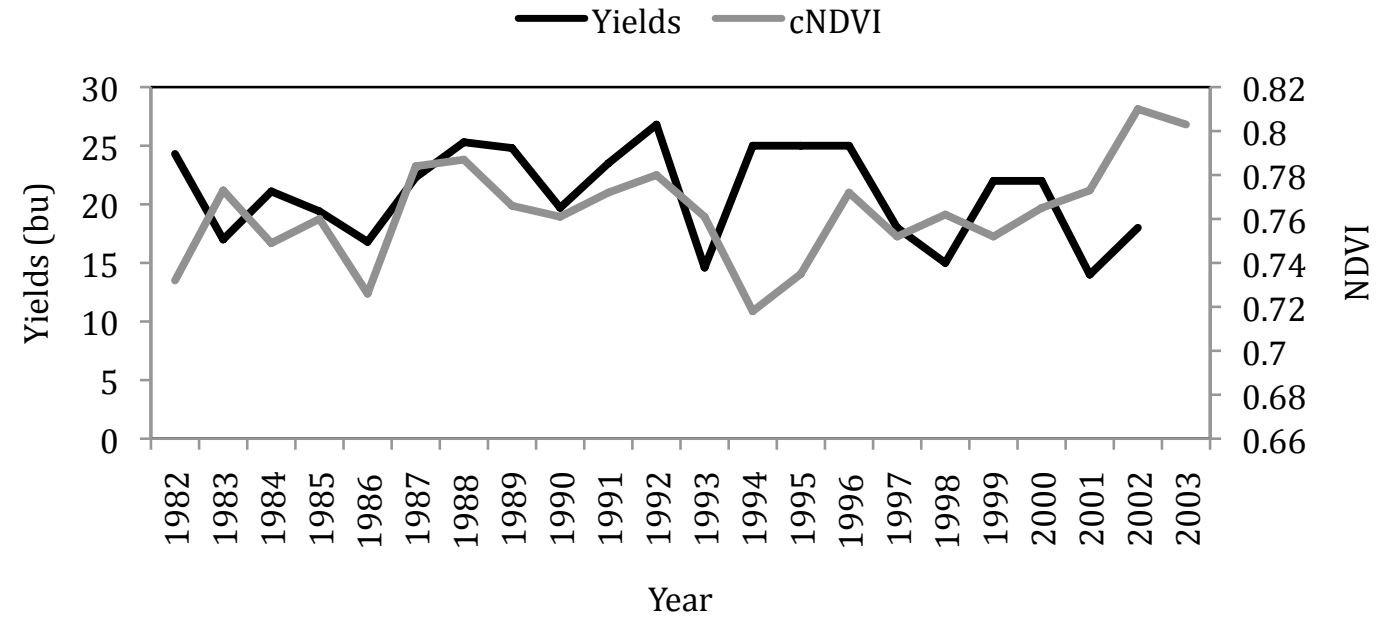

Figure 4.2. Soy Yields and NDVI for Saluda, SC 


\section{Case Study: Windsor, IL}

Windsor, IL provides a different perspective on the relationship between NDVI and crop yields, than what we saw in Saluda, SC. In the case of this station, NDVI has a positive coefficient for both corn and soybean yields. The relationship is not statistically significant in the case of either crop. However, the model does have a good fit for both crops, with $R^{2}$ values of 0.7346 and 0.6746 for corn and soybeans, respectively. F-tests also show the model to have explanatory power. Signs on the coefficients of the other variables are consistent for both crops at this location, and are largely as expected, with the exception of the negative coefficient on $N D V l^{2}$. These results are shown in Tables 4.6 and 4.7. 
Table 4.6. Results for Yields Regressed on NDVI and meteorological variables, for corn at Windsor, IL

\begin{tabular}{|c|cccccc|}
\hline Variable & $\beta$ & Std. err. & $\mathrm{t}$ & P-value & \multicolumn{2}{c|}{$95 \%$ Confidence Int. } \\
\hline$N D V I$ & 2518.3 & 2868.266 & 0.88 & 0.397 & -3731.114 & 8767.715 \\
$C P$ & 9.5 & 48.91393 & 0.19 & 0.849 & -97.05187 & 116.0967 \\
$G D D$ & -.5732 & .9884681 & -0.58 & 0.573 & -2.72687 & 1.580504 \\
$N D V I^{2}$ & -1328.2 & 2101.81 & -0.63 & 0.539 & -5907.697 & 3251.202 \\
$C P^{2}$ & .3865585 & .6925499 & 0.56 & 0.587 & -1.122378 & 1.895495 \\
$G D D^{2}$ & .0001308 & .000685 & 0.19 & 0.852 & -.0013617 & .0016232 \\
$C P N D V I$ & -22.4185 & 60.28347 & -0.44 & 0.716 & -153.7649 & 108.9278 \\
$G D D N D V I$ & .6550718 & 1.121387 & -0.37 & 0.570 & -1.78822 & 3.098363 \\
$C P G D D$ & -.012735 & .0291876 & 0.58 & 0.670 & -.0763288 & .0508597 \\
\hline$R^{2}$ & & & & & & 0.7346 \\
\hline
\end{tabular}

Table 4.7. Results for Yields regressed on NDVI and meteorological variables, for soybeans at Windsor, IL

\begin{tabular}{|c|cccccc|}
\hline Variable & $\beta$ & Std. err. & $\mathrm{t}$ & P-value & \multicolumn{2}{c|}{$95 \%$ Confidence Int. } \\
\hline$N D V I$ & 515.2734 & 724.6016 & 0.71 & 0.491 & -1063.498 & 2094.045 \\
$C P$ & 4.313011 & 15.71782 & 0.27 & 0.788 & -29.93318 & 38.5592 \\
$G D D$ & -.0790699 & .3274609 & -0.24 & 0.813 & -.7925459 & .6344062 \\
$N D V I^{2}$ & -244.5751 & 557.5881 & -0.44 & 0.669 & -1459.455 & 970.305 \\
$C P^{2}$ & .0507554 & .2178401 & 0.23 & 0.820 & -.4238775 & .5253882 \\
$G D D^{2}$ & .0000588 & .0002837 & 0.21 & 0.839 & -.0005595 & .000677 \\
$C P N D V I$ & -6.399578 & 18.31468 & -0.35 & 0.733 & -46.30383 & 33.50468 \\
$G D D N D V I$ & .0670142 & .3631861 & 0.18 & 0.857 & -.7243004 & .8583289 \\
$C P G D D$ & -.0021712 & .012079 & -0.18 & 0.860 & -.0284891 & .0241468 \\
\hline$R^{2}$ & & & & & & 0.6746 \\
\hline
\end{tabular}


Again, it is necessary to find the marginal effect of $N D V I, c p$, and gdd on NDVI to understand the relationships depicted by these regression results. The yield function for corn in Windsor, IL is given by (Equation 4.6)

Yield $=-949.01+2518.3 N D V I+9.5224 c p-0.5732 g d d-1328.2 N D V I^{2}+0.38656 c p^{2}+0.00013 g d d^{2}$ $-22.4185 c p N D V I+0.65507 g d d N D V I-0.012735 c p g d d$

and the equation for soy yield is as follows

\section{(Equation 4.7)}

Yield $=-207.2024+515.2734 N D V I+4.313 c p-0.0791 g d d-244.5751 N D V I^{2}+.0507554 c p^{2}+.000059 g d d^{2}$ $-6.3996 c p N D V I+0.067 g d d N D V I-0.0022 c p g d d$

Evaluating the respective partial derivatives of this equation at the mean values for each variable, gives $\frac{\partial Y \text { ield }}{\partial N D V I}=1879.366$ and $\frac{\partial Y \text { ield }}{\partial N D V I}=247.98$ for corn and soybeans, respectively. While this shows the anticipated positive relationship between NDVI and yields, the marginal effect of $c p$ on yields was negative in both the case of corn (-7.25) and soybeans (-0.8683). However, this is not unreasonable given that cumulative seasonal precipitation does not necessarily reflect periods of drought during critical growth phases, or large amounts of precipitation occurring during a short time period, which may not be beneficial to crop growth.

Examining the relationship between NDVI and yields at Windsor, IL by looking at graphs of the time series data (Figures 4.3. and 4.4.), the relationship appears to be somewhat stronger than was the case for Saluda, 
$\mathrm{SC}$, but consistency is still lacking. In looking at corn yields, NDVI captures drops in yields in 1984, 1988, 1991, 1996, and 2002. However, it also experiences a drop during a year of increased yields, in 1994, 1998, and 2000. Additionally NDVI increases, during the drop in yields in 1983.

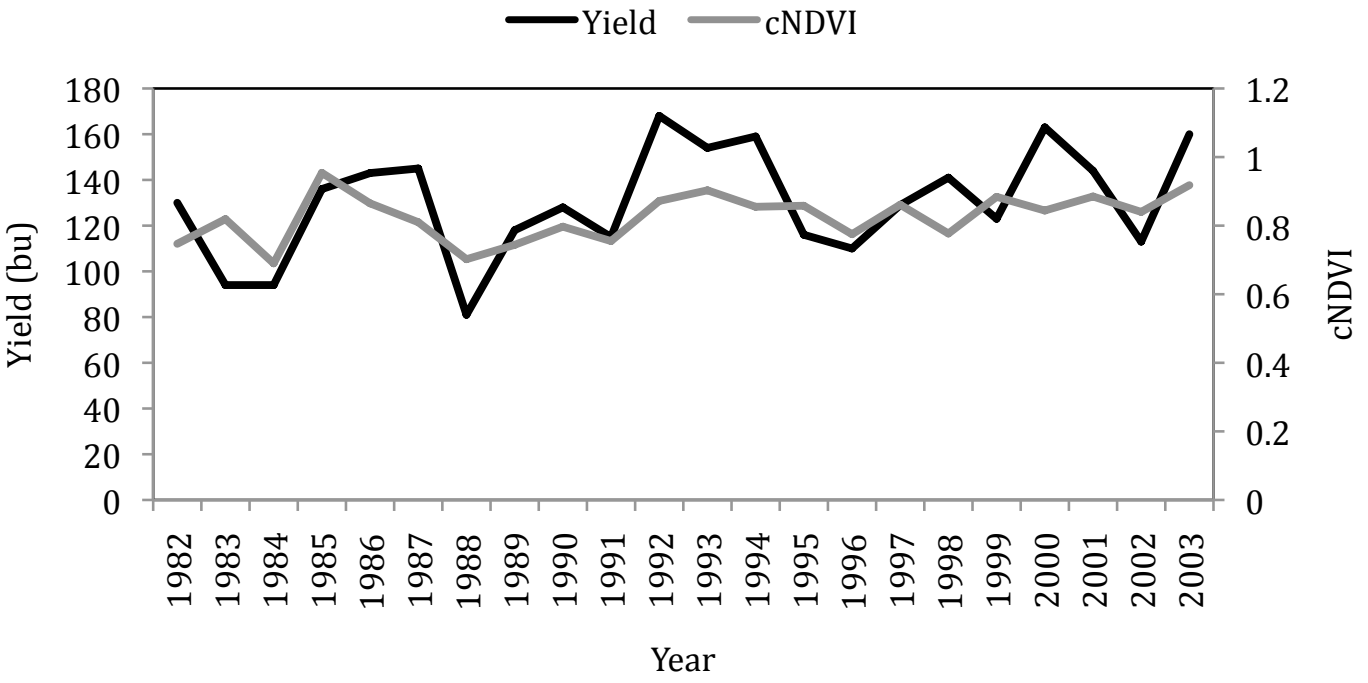

Figure 4.3 Corn Yields and NDVI for Windsor, IL

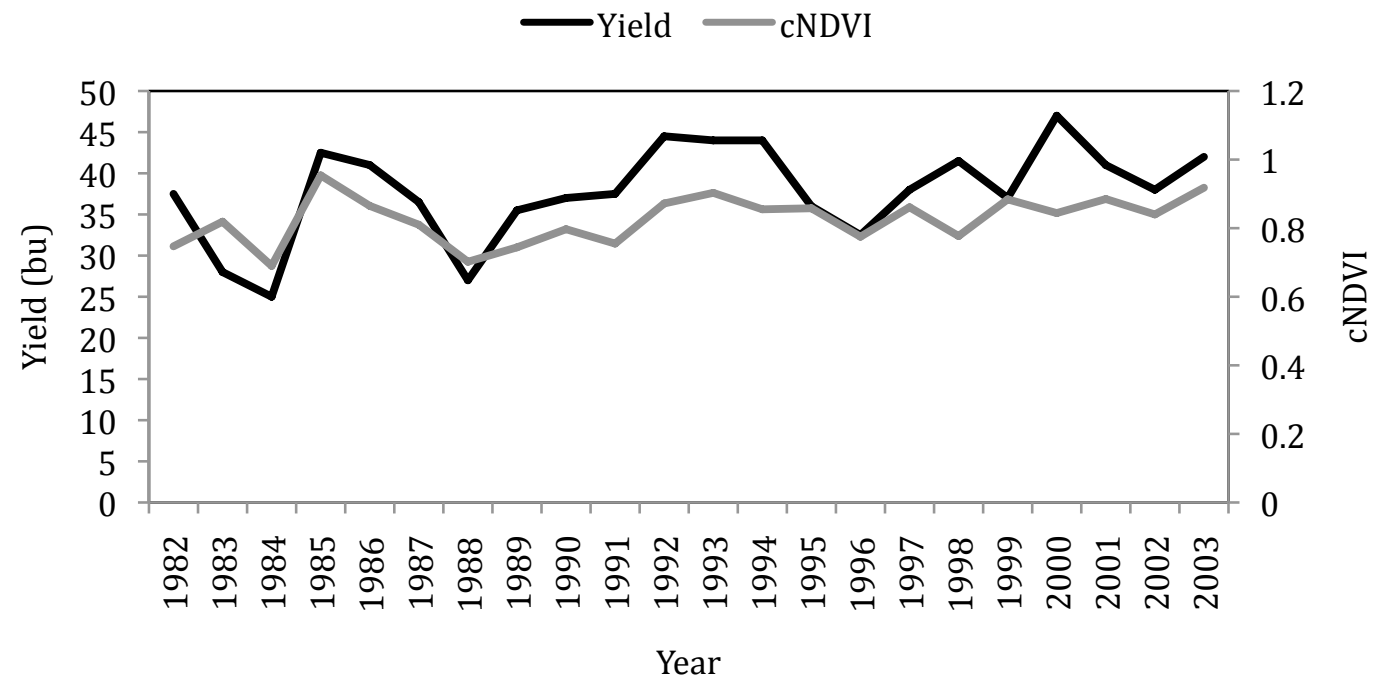

Figure 4.4 Soy Yields and NDVI for Windsor, IL 
Similarly, soybean yields and NDVI in Windsor, IL appear to correspond for some fragments of time, yet ultimately show an inconsistent relationship. This can be seen with the increased NDVI values in 1983 and1999, both of which correspond to a decrease in yields for these years. Additionally, we see a drop in NDVI in1998 and 2000, both of which were years of increased soybean yield.

\section{NDVI and Crop Indemnities}

\section{A simple index-based insurance contract using NDVI values}

Though my regression analysis provided little evidence of a relationship between NDVI and crop yields that could be considered reliable for a range of locations, ultimately the question at hand is can years of anomalously great crop loss be captured by a NDVI-based trigger mechanism. To test this, I assessed the effectiveness of a simple NDVI-based contract in addressing major loss events by performing a Historical Burn Analysis. The contract would pay out when seasonal NDVI values were below a trigger value of average $N D V I-\left(0.25^{\star}\right.$ standard deviation), where average $N D V I$ is the mean NDVI for the growing season. This design is similar to the design implemented by USDA RMA's Pasture, Forage and Rangeland program, where losses are measured as the difference between expected NDVI for a parcel of land and actual NDVI for that parcel during a critical growing period, and a trigger value is decided upon to render a payout.

For this example, I chose a quarter of a standard deviation below the average NDVI value for the location, because it provided a uniform rule that resulted in a payout frequency typical for an index-based agricultural insurance 
product (around 20-35\%) for each of the locations aside from a select few. I confined the contract to NDVI values during the growing season defined as May 1 to August 15, as this was the closest to representing critical growing periods that could be applied across a wide variety of locations, each growing different crops at different times depending on their unique growing seasons. I used a Historical Burn Analysis to evaluate this contract for corn at each of the 25 locations, and for wheat, hay and soybeans at the locations where data was available.

With the exception of five stations (Dublin, GA, Beaverdam, KY, Eltopia, WA, and Fredonia, NY), all stations had a payout frequency ranging from 20 to 25 percent. When evaluating the contract in relation to corn yields, $0 \%$ to $71 \%$ of these payouts occurred in the lowest quarter of crop yields. 86 to $20 \%$ occurred in the lowest half of crop yields. Rockrapids, IA was the station where the contract best succeeded in capturing extreme crop loss events, with $71 \%$ of payouts falling in years in the lowest quarter of crop yields, and $86 \%$ occurring in years in the lowest half of crop yields. While, in Heppner, OR the contract failed to detect any of the years in the lowest quarter of crop yields, and captured only one of the events in the lowest half of crop yields. Table 4.8 presents general statistics on the relationship between payouts and indemnity events for corn crops at all of the locations. 
Table 4.8 Payouts for NDVI-based insurance contract for corn (trigger at 0.5 standard deviations below average seasonal maximum NDVI value)

\begin{tabular}{|c|c|c|c|}
\hline Station & Payout frequency & Worst $1 / 4$ of years & Worst $1 / 2$ of years \\
\hline Algona, IA & $25 \%$ & $40 \%$ & $40 \%$ \\
Rockrapids, IA & $35 \%$ & $71 \%$ & $86 \%$ \\
Saluda, SC & $25 \%$ & $20 \%$ & $40 \%$ \\
Dublin, GA & $40 \%$ & $38 \%$ & $38 \%$ \\
Booneville, MS & $30 \%$ & $50 \%$ & $67 \%$ \\
Watervalley, MS & $20 \%$ & $50 \%$ & $75 \%$ \\
Batesville, MS & $30 \%$ & $50 \%$ & $67 \%$ \\
Yazoo City, MS & $40 \%$ & $13 \%$ & $38 \%$ \\
Angelica, NY & $35 \%$ & $14 \%$ & $29 \%$ \\
Riverhead, NY & $35 \%$ & $14 \%$ & $43 \%$ \\
Fredonia, NY & $50 \%$ & $30 \%$ & $50 \%$ \\
DuQUoin, IL & $25 \%$ & $40 \%$ & $80 \%$ \\
Minonk, IL & $25 \%$ & $60 \%$ & $80 \%$ \\
Ardmore, SD & $30 \%$ & $50 \%$ & $50 \%$ \\
Windsor, IL & $35 \%$ & $57 \%$ & $71 \%$ \\
WhiteHall, IL & $30 \%$ & $67 \%$ & $83 \%$ \\
Beaverdam, KY & $40 \%$ & $50 \%$ & $75 \%$ \\
Providence, KY & $30 \%$ & $33 \%$ & $67 \%$ \\
Farmville, VA & $30 \%$ & $33 \%$ & $67 \%$ \\
Heppner, OR & $25 \%$ & $0 \%$ & $20 \%$ \\
Eltopia, WA & $50 \%$ & $50 \%$ & $80 \%$ \\
Menomonie, WA & $25 \%$ & $60 \%$ & $60 \%$ \\
Arlington, WI & $35 \%$ & $43 \%$ & $71 \%$ \\
Sellingsgrove,PA & $30 \%$ & $17 \%$ & $33 \%$ \\
Montrose, PA & $35 \%$ & $29 \%$ & $43 \%$ \\
\hline
\end{tabular}

Figures 4.5 and 4.6 depict the timing of payouts for the NDVI-based insurance contract for corn for Saluda, SC and Windsor, IL. In the case of Saluda, SC only one of the five payouts occurred in a year exhibiting anomalously low crop yields. The other four payouts all occur in years with above-average corn yields. 


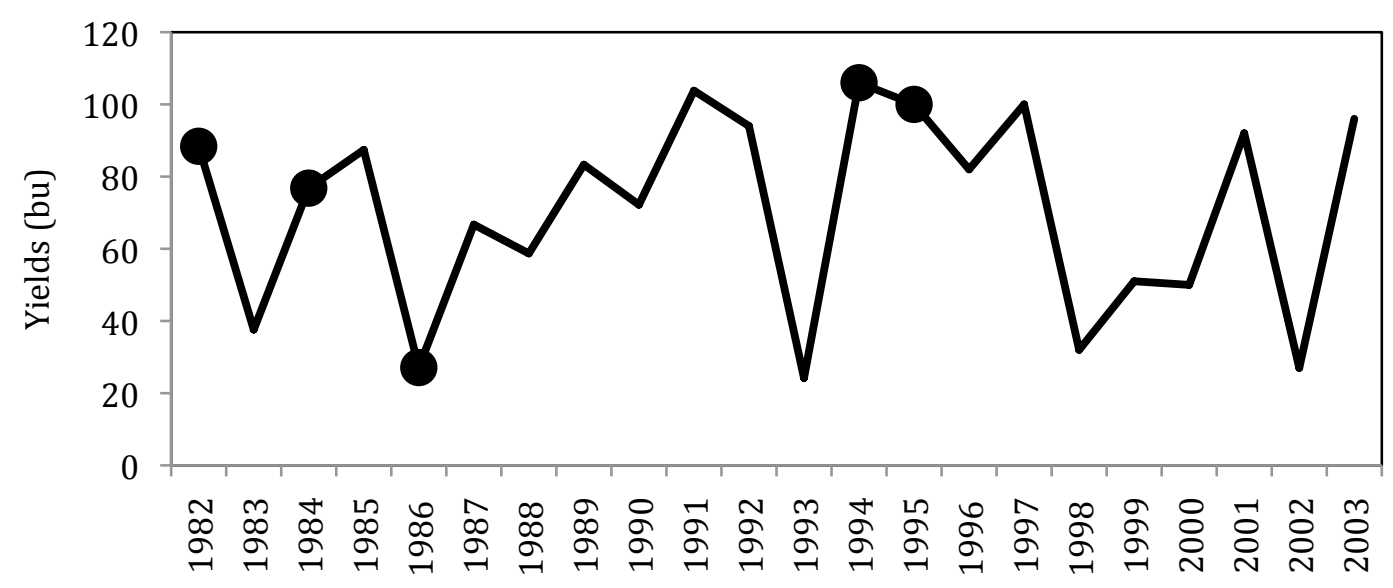

Year

Figure 4.5. Corn Yields for Saluda, SC and payouts using NDVI-based insurance contract

The contract is more successful at targeting loss in the case of

Windsor, IL. Here, no payouts occur in the years with the highest yields, and loss events, such as low crop yields in 1984 and 1988, are successfully captured by the contract. Additionally, years such as 1989, 1991, and 1996, which are in the lower half of yields, receive a payout. However, the contract does fail to capture 1983, a year of anomalously low yields.

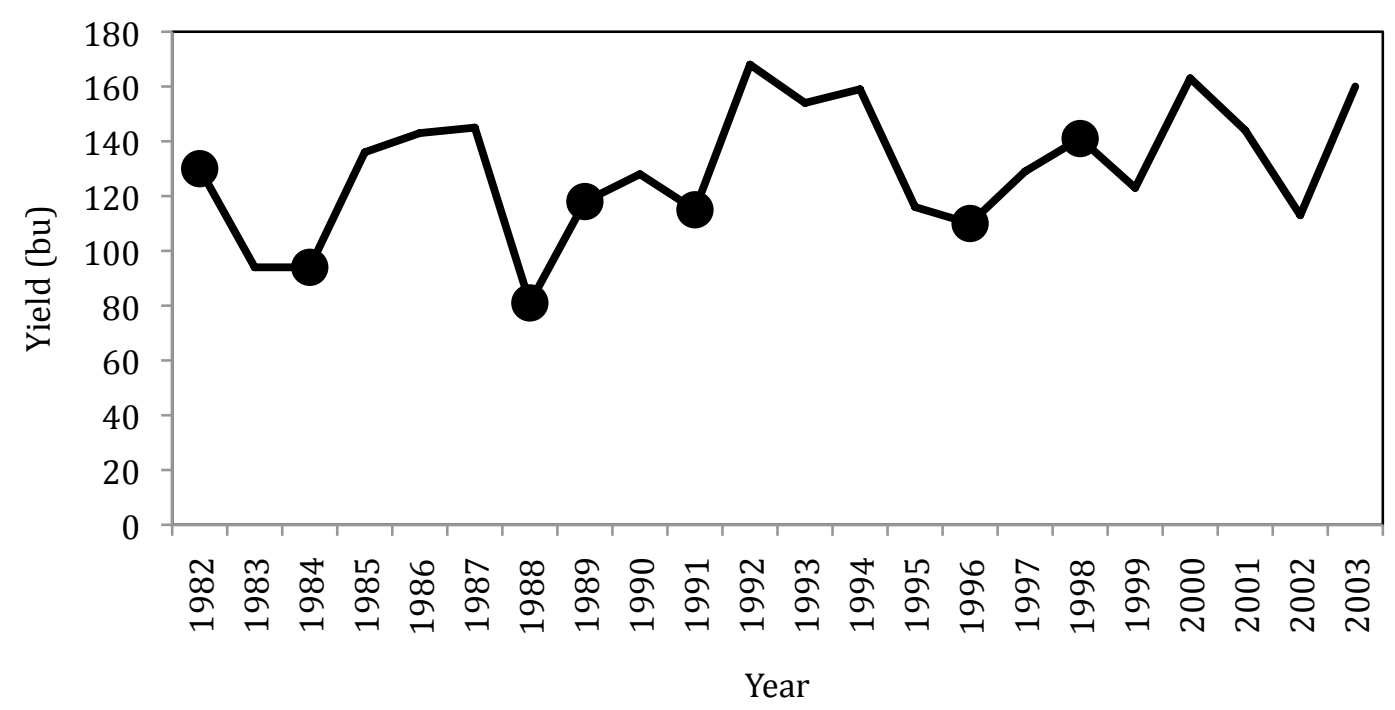

Figure 4.6. Corn Yields for Windsor, IL and payouts using NDVI-based insurance contract 
Comparing contract payouts to hay, wheat, and soybean yields had similar results, with 0 to $80 \%$ of payouts occurring in the lowest quarter of wheat yields, 0 to $57 \%$ in the lowest quarter of hay yields, and 17 to $60 \%$ in the lowest quarter of soybean yields. The contract was best at capturing extreme wheat crop loss in Menomonie, WA ( $80 \%$ of payouts in the lowest quarter of yields, $100 \%$ in the lowest half). For hay yields, payouts were most often triggered in years with the greatest losses in Rockrapids, IA and Angelica, NY, where $57 \%$ of payouts were in the lowest quarter of yields, and $86 \%$ were in the lowest half for both cases. Algona, IA had the greatest percentage of payouts in the worst quarter of soy yields $(60 \%)$, yet also had $40 \%$ of payouts in years in the highest half of soy yields, while Rock Rapids, IA and Windsor, IL had $86 \%$ of payouts occurring in the lowest half of crop yields.

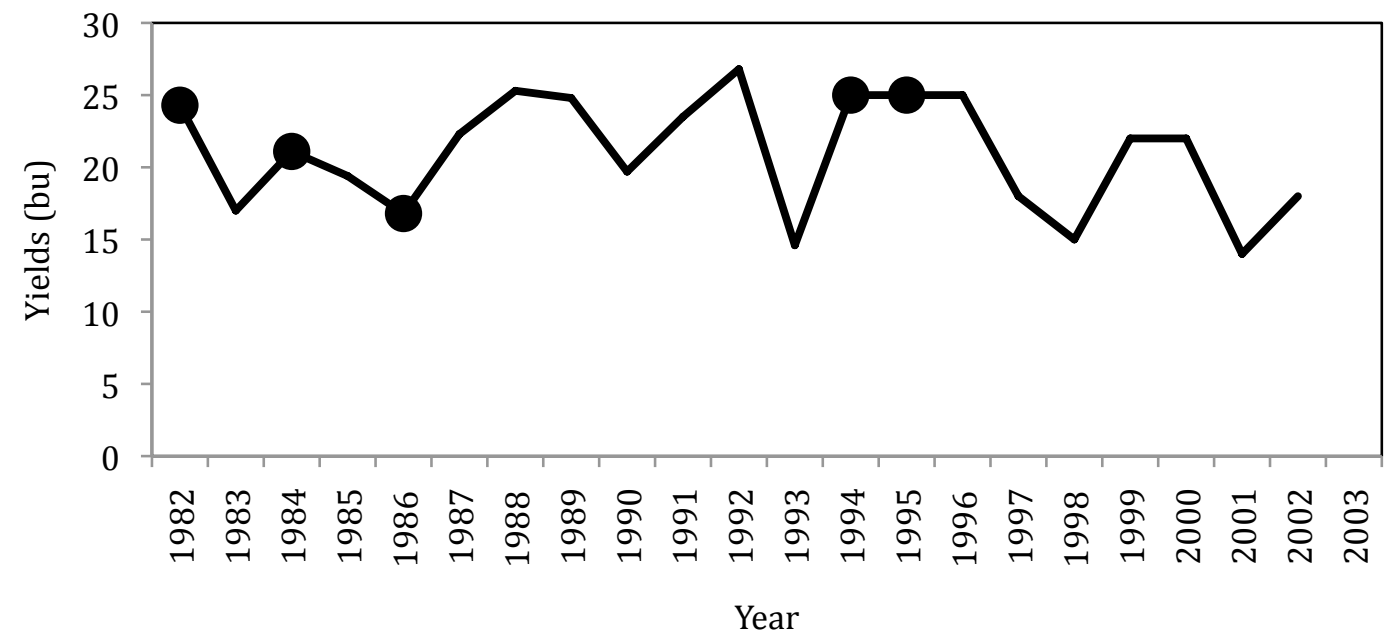

Figure 4.7. Soy Yields for Saluda, SC and payouts using NDVI-based insurance contract 
The timing for the NDVI-based soybean contract for Saluda, SC is presented in Figure 4.7. As was the case of the corn contract in Saluda, only one payout occurs in a year of anomalously low crop yields. Three occur in years in the highest half of yields.

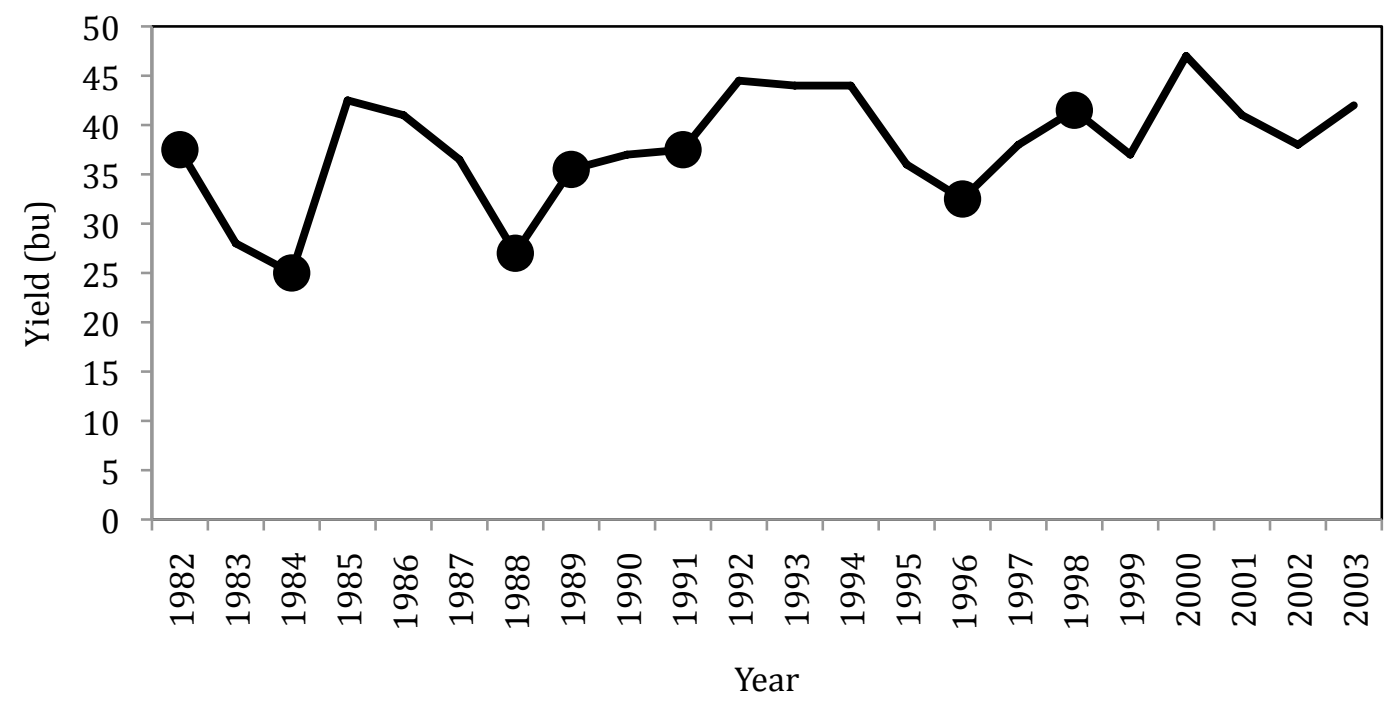

Figure 4.8. Soy yields for Windsor, IL and payouts using NDVI-based insurance contract

The NDVI-based contract for soybeans performed better at the Windsor, IL location. All but one of the payouts (1998) occur in years in the lowest half of yields, and four of the seven payouts $(1984,1988,1989$ and 1996) occur in years in the lowest quarter of yields. Figure 4.8 shows the timing of these events. 


\section{CHAPTER 5}

Conclusion and Suggestions for Future research

\section{NDVI and Precipitation}

The relationship between raw NDVI data and precipitation differs depending on location, and likely many other factors outside the scope of this model. My results show NDVI to have a positive relationship with precipitation in some locations, while exhibiting a negative relationship in others. This is in line with both those studies finding a significant positive relationship between NDVI and precipitation (Wang et al. 2003, Thoma et al., Kennedy 1989), and those finding weaker, highly variable, or negative relationships between NDVI and precipitation (du Plessis 1989, Yang et al. 1998, Farrar et al. 1994). The quadratic form used in this study shows precipitation to be a significant variable affecting NDVI in very few locations within our study sample. This is in line with the literature showing a high variability in the strength of the relationship between precipitation and NDVI (du Plessis et al.1999, Hielkema et al.1986, Groton 1991).

It is important to note that prior studies finding strong relationships between NDVI and rainfall were mostly working in smaller regions, with study sites often restricted to one state or county. Additionally, most were working with a shorter time series of data, many comparing data spanning only two to three years. 


\section{NDVI and Extreme Heat}

Results from this study show the relationship between NDVI and extreme heat to be highly variable among locations. Furthermore, the relationship between NDVI and extreme heat is likely not constant for the growing season. Wang et al. (2003) found temperature to be negatively correlated with NDVI during the middle of the growing season, while it was positively correlated with NDVI at the beginning and end. Such changes in the relationship between temperature and NDVI throughout the growing season may account for some of the variation in signs on coefficients for the $G D D$ and $G D D^{2}$ variables. Additionally, prior studies have found NDVI to be both positively and negatively correlated with temperature interannually (Yang et al., Wang et al., Zhou et al.). Given the assorted locations covered by this study, it is not surprising that my results agree with both findings.

The strength of the relationship between NDVI and extreme heat also differs depending on location. While extreme heat was a significant variable in some locations, in most cases it was not. This is likely the result of the interplay of many factors including interaction effects between temperature and precipitation, soil water content, potential evapotranspiration, and vegetation type. Though a complex crop model incorporating such factors may be able to explain the relationship between NDVI and temperature, NDVI alone does not reliably reflect instances of extreme heat across a wide variety of locations. This is not to say that NDVI cannot capture vegetation stress resulting from extreme heat in particular geographic areas. 


\section{NDVI and Crop Yields}

The results of this study did not find a consistent relationship between NDVI and crop yields across study sites. Though the model had low coefficients of determination in some locations, in most it was able to effectively account for a large proportion of the variation in NDVI. Though all the sites in this study were comprised predominantly of farmland with little to no irrigation, many locations are growing multiple crops, others consist primarily of pasture, and many contain forested areas. I did not include data on technology changes or crop rotation, which have been evidenced to make NDVI less reliable in detecting trends in vegetation conditions over time (Atwood et al. 2005). Furthermore, using county level data may have weakened expressed relationships, as there are certain to be discrepancies between NDVI pixel location and county lines. Nonetheless, my results indicate that the relationship between NDVI and crop yields is not strong enough at the single-pixel level, such that it would not be disturbed or lost amidst changes in these sorts of factors.

Several studies have found NDVI is most effective when consolidating pixels covering an expansive area of land (de Plessis 1999, Rowley et al. 2007). Some have found NDVI to be most successful at capturing vegetative condition when estimated over an extended period of time (15 months, in the case of Wang et al. 2003) or when smoothing techniques are employed (du Plessis 1999). While these papers point to a strong underlying relationship between NDVI and vegetation condition, my results suggest NDVI is not particularly useful when looking at the smaller-scale, seasonal information relevant to index-based crop insurance products. This is consistent with other studies assessing the possibilities of using NDVI for index-based 
agricultural insurance, which found significant differences between farm-level experiences and NDVI values (Rowley et al. 2007, Makaudze and Miranda 2009). This is further evidenced by the weak ability of NDVI to capture years of extreme crop loss across locations, as seen by the historical Burn Analysis presented in Chapter 7.

\section{General Conclusions on NDVI and Meteorological Variables}

Relationships between NDVI and precipitation and extreme heat are highly variable in locations throughout the United States. This study was not able to find a reliable relationship between NDVI and precipitation or between NDVI and extreme heat that would justify the use of NDVI as a substitute for meteorological data in the development of index-based insurance products without careful consideration of in situ data and local calibration. While NDVI may be able to reliably reflect rainfall and extreme heat patterns in some locations, notably grasslands and pastures, the relationship is not as clear in mixed vegetation environments including cropland.

\section{NDVI and index-based crop insurance}

The relationship between seasonal maximum NDVI and crop indemnities, as well as seasonal integrated NDVI and crop indemnities is highly variable, and mostly weak for the sample of locations this study explored. From this, I conclude NDVI is not a product that can be applied broadly to estimate crop loss without location specific calibration reliant on in situ data. 


\section{Directions for Further Research}

More research is needed exploring the time period and area size best suited to maximizing the strength of the relationship between NDVI, meteorological variables, and crop yields. Examining the relationship between NDVI during critical stages of crop growth and crop yields may provide useful information for insurance design. A better understanding of the connection between crop productivity and the condition of surrounding natural vegetation may also help to make NDVI a more useful tool in insurance product design, as it would allow the NDVI values to be aggregated over a larger area, a condition shown to contribute to more accurate NDVI responses.

As projects such as the Millennium Development Villages across regions of Africa have determined NDVI to effectively capture periods of stress undergone by natural vegetation and have found these periods to correspond directly to severe crop loss, setting aside plots of natural vegetation to use as markers for NDVI measurements may provide a method of eliminating some of the noise in NDVI measurements created by changes in land management practices and crop rotations. Sizeable plots of native vegetation, measuring $10 \mathrm{~km} \times 10 \mathrm{~km}$ for example, may provide a stable measure of the fluctuations in vegetation condition caused by weather-related stress, which would also be affecting local crop yields. Such plots would in effect provide a form of localcalibration of NDVI data. Exploring the efficacy of this method in diverse regions with a multitude of agricultural practices, such as China and the United States would provide great insight into how NDVI can best be used in indexbased agricultural insurance design. 


\section{APPENDIX A}

\section{Regression results for NDVI and meteorological variables}

\section{Ada, OK}

Linear regression

$\begin{array}{llr}\text { Number of obs } & & 152 \\ \text { F }(32,119) & 2.58 \\ \text { Prob F } & =0001 \\ \text { R-squared } & =0.4291 \\ \text { Root MSE } & = & .04287\end{array}$

\begin{tabular}{|c|c|c|c|c|c|c|}
\hline ndvi_adaok & Coef. & $\begin{array}{c}\text { Robust } \mathrm{HC} 3 \\
\text { Std. Err. }\end{array}$ & $t$ & $P>|t|$ & [ $95 \%$ Conf. & Interval] \\
\hline year_1983 & .0394435 & .0213864 & 1.84 & 0.068 & -.0029037 & .0817907 \\
\hline year_1984 & -.0427077 & .0242026 & -1.76 & 0.080 & -.0906312 & .0052158 \\
\hline year_1985 & .0128649 & .0313944 & 0.41 & 0.683 & -.0492992 & .075029 \\
\hline year 1986 & -.0209095 & .0284192 & -0.74 & 0.463 & -.0771823 & .0353633 \\
\hline year_1987 & .0313425 & .0291725 & 1.07 & 0.285 & -.0264219 & .089107 \\
\hline year 1988 & -.0094267 & .0267219 & -0.35 & 0.725 & -.0623387 & .0434854 \\
\hline year 1989 & -.0106469 & .0293555 & -0.36 & 0.717 & -.0687737 & .0474799 \\
\hline year_1990 & -.0029997 & .0243532 & -0.12 & 0.902 & -.0512214 & .045222 \\
\hline year_1991 & .0047555 & .0231668 & 0.21 & 0.838 & -.0411171 & .0506281 \\
\hline year 1992 & .0453441 & .0285352 & 1.59 & 0.115 & -.0111584 & .1018467 \\
\hline year 1993 & .0100954 & .0234531 & 0.43 & 0.668 & -.0363441 & .0565349 \\
\hline year 1994 & .0259888 & .0221882 & 1.17 & 0.244 & -.0179461 & .0699236 \\
\hline year_1995 & .0196404 & .02547 & 0.77 & 0.442 & -.0307928 & .0700736 \\
\hline year_1996 & .0315804 & .0298846 & 1.06 & 0.293 & -.0275942 & .0907549 \\
\hline year_1997 & .0191106 & .0269941 & 0.71 & 0.480 & -.0343405 & .0725617 \\
\hline year_1998 & .0086192 & .0442186 & 0.19 & 0.846 & -.0789381 & .0961765 \\
\hline year_1999 & -.0257244 & .0417958 & -0.62 & 0.539 & -.1084843 & .0570355 \\
\hline year_2000 & .0300921 & .0220915 & 1.36 & 0.176 & -.0136513 & .0738356 \\
\hline year_2001 & .0009579 & .0290449 & 0.03 & 0.974 & -.0565539 & .0584698 \\
\hline year_2002 & .0151968 & .0252806 & 0.60 & 0.549 & -.0348613 & .0652549 \\
\hline year_2003 & -.0286625 & .0261299 & -1.10 & 0.275 & -.0804023 & .0230774 \\
\hline $\operatorname{may} 1631$ & .0129358 & .0142785 & 0.91 & 0.367 & -.0153371 & .0412086 \\
\hline jun115 & .0223834 & .0177979 & 1.26 & 0.211 & -.0128581 & .057625 \\
\hline jun 1630 & .0109258 & .0233547 & 0.47 & 0.641 & -.0353189 & .0571704 \\
\hline jul115 & .0020891 & .025836 & 0.08 & 0.936 & -.0490689 & .053247 \\
\hline jul1631 & -.0007733 & .0274087 & -0.03 & 0.978 & -.0550452 & .0534986 \\
\hline $\operatorname{aug} 115$ & -.0366179 & .0278795 & -1.31 & 0.192 & -.091822 & .0185863 \\
\hline cp_adaok & .0043377 & .0089065 & 0.49 & 0.627 & -.0132981 & .0219736 \\
\hline gdd_adaok & .0001661 & .0003552 & 0.47 & 0.641 & -.0005373 & .0008694 \\
\hline c $\bar{p} 2$ ada & -.0014831 & .000979 & -1.51 & 0.132 & -.0034217 & .0004554 \\
\hline gdd2_ada & $-1.15 e-06$ & $1.20 e-06$ & -0.96 & 0.339 & $-3.53 e-06$ & $1.22 e-06$ \\
\hline cpgdd_ada & .0000109 & .0000576 & 0.19 & 0.850 & -.0001032 & .000125 \\
\hline _cons & .6288807 & .0306546 & 20.52 & 0.000 & .5681814 & .6895799 \\
\hline
\end{tabular}




\section{Algona, IA}

Linear regression

$\begin{array}{llr}\text { Number of obs } & = & 152 \\ \mathrm{~F}(32, \quad 119) & =53.89 \\ \text { Prob }>\mathrm{F} & =0.0000 \\ \text { R-squared } & =0.9209 \\ \text { Root MSE } & =.05276\end{array}$

\begin{tabular}{|c|c|c|c|c|c|c|}
\hline ndvi_algon a & Coef. & $\begin{array}{l}\text { Robust } \mathrm{HC} \\
\text { Std. Err. }\end{array}$ & $t$ & $\mathrm{P}>|\mathrm{t}|$ & [95\% Conf. & Interval] \\
\hline 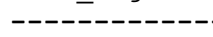 & --------- & ---------- & 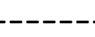 & ------2 & -------- & --------1 \\
\hline year_1983 & .0631536 & .041626 & 1.52 & 0.132 & -.01927 & .1455772 \\
\hline year_1984 & .0248295 & .0313507 & 0.79 & 0.430 & -.0372481 & .0869071 \\
\hline year 1985 & .0867977 & .0322878 & 2.69 & 0.008 & .0228647 & .1507308 \\
\hline year_1986 & .0915404 & .0347672 & 2.63 & 0.010 & .022698 & .1603829 \\
\hline year_1987 & .1327934 & .0474371 & 2.80 & 0.006 & .0388631 & .2267237 \\
\hline year_1988 & .140852 & .0372587 & 3.78 & 0.000 & .0670761 & .214628 \\
\hline year_1989 & .0585697 & .0318819 & 1.84 & 0.069 & -.0045597 & .1216991 \\
\hline year_1990 & .0411427 & .0337416 & 1.22 & 0.225 & -.0256692 & .1079545 \\
\hline year_1991 & -.030298 & .0449792 & -0.67 & 0.502 & -.1193612 & .0587653 \\
\hline year_1992 & .0763801 & .0381436 & 2.00 & 0.048 & .0008519 & .1519083 \\
\hline year_1993 & -.0116888 & .0348495 & -0.34 & 0.738 & -.0806942 & .0573166 \\
\hline year_1994 & .1370987 & .0409116 & 3.35 & 0.001 & .0560897 & .2181078 \\
\hline year_1995 & .0936568 & .032826 & 2.85 & 0.005 & .028658 & .1586556 \\
\hline year_1996 & .0468628 & .039944 & 1.17 & 0.243 & -.0322303 & .1259559 \\
\hline year_1997 & .0952371 & .034762 & 2.74 & 0.007 & .0264048 & .1640694 \\
\hline year_1998 & .1154962 & .0353675 & 3.27 & 0.001 & .045465 & .1855274 \\
\hline year_1999 & -.0686399 & .0558113 & -1.23 & 0.221 & -.1791518 & .0418721 \\
\hline year_2000 & .0826229 & .0406787 & 2.03 & 0.044 & .002075 & .1631708 \\
\hline year_2001 & .0580147 & .0346757 & 1.67 & 0.097 & -.0106467 & .1266762 \\
\hline year_2002 & .0819909 & .0359399 & 2.28 & 0.024 & .0108263 & .1531556 \\
\hline year_2003 & .0658559 & .0391773 & 1.68 & 0.095 & -.011719 & .1434308 \\
\hline may 1631 & .073524 & .0166653 & 4.41 & 0.000 & .040525 & .1065229 \\
\hline jun115 & .1399256 & .0142536 & 9.82 & 0.000 & .1117022 & .1681491 \\
\hline jun 1630 & .2290266 & .0187385 & 12.22 & 0.000 & .1919224 & .2661308 \\
\hline jul115 & .3358135 & .0140273 & 23.94 & 0.000 & .308038 & .3635891 \\
\hline jul1631 & .4022277 & .0150515 & 26.72 & 0.000 & .3724242 & .4320312 \\
\hline $\operatorname{aug} 115$ & .422233 & .0195217 & 21.63 & 0.000 & .3835781 & .460888 \\
\hline cp_algonaia & .0007647 & .0122482 & 0.06 & 0.950 & -.0234879 & .0250173 \\
\hline gdd_algonaia & -.0008058 & .0013247 & -0.61 & 0.544 & -.003429 & .0018173 \\
\hline cp 2 algona & -.0002153 & .0022706 & -0.09 & 0.925 & -.0047113 & .0042806 \\
\hline gdd2_algona & $3.79 e-06$ & .0000118 & 0.32 & 0.749 & -.0000196 & .0000272 \\
\hline cpgdd_algona & $-2.68 e-06$ & .000396 & -0.01 & 0.995 & -.0007868 & .0007814 \\
\hline _cons & .3328285 & .0361729 & 9.20 & 0.000 & .2612026 & .4044545 \\
\hline
\end{tabular}




\title{
Harlan, IA
}

\author{
Linear regression
}

$\begin{array}{llr}\text { Number of obs } & = & 152 \\ \text { F }(32,119) & 65.70 \\ \text { Prob F } & =0000 \\ \text { R-squared } & =0.9221 \\ \text { Root MSE } & = & .05271\end{array}$

\begin{tabular}{|c|c|c|c|c|c|c|}
\hline ndvi_harla a & Coef. & $\begin{array}{l}\text { Robust } \mathrm{HC} 3 \\
\text { Std. Err. }\end{array}$ & $t$ & $P>|t|$ & [95\% Con & Int \\
\hline year 1983 & .0409646 & .0295164 & 1.39 & 0.168 & -.0174808 & .09941 \\
\hline year 1984 & .0155336 & .0283862 & 0.55 & 0.585 & -.040674 & .0717412 \\
\hline year 1985 & .0553047 & .0278631 & 1.98 & 0.049 & .0001331 & .1104764 \\
\hline year_1986 & .0586671 & .0311813 & 1.88 & 0.062 & -.0030751 & .1204093 \\
\hline year 1987 & .0968252 & .0383873 & 2.52 & 0.013 & .0208144 & .1728359 \\
\hline year 1988 & .1117614 & .0357534 & 3.13 & 0.002 & .0409662 & .1825567 \\
\hline year 1989 & .0258346 & .0272867 & 0.95 & 0.346 & -.0281958 & .0798651 \\
\hline year_1990 & .0355605 & .0286514 & 1.24 & 0.217 & -.021172 & .0922931 \\
\hline year_1991 & .0981805 & .0327092 & 3.00 & 0.003 & .033413 & .162948 \\
\hline year 1992 & .0430454 & .0346576 & 1.24 & 0.217 & -.0255802 & .111671 \\
\hline year 1993 & .0266755 & .036312 & 0.73 & 0.464 & -.0452258 & .0985768 \\
\hline year-1994 & .1169531 & .0367396 & 3.18 & 0.002 & .044205 & .1897013 \\
\hline year-1995 & .0262176 & .0379576 & 0.69 & 0.491 & -.0489422 & .1013775 \\
\hline year 1996 & .0063734 & .0306385 & 0.21 & 0.836 & -.0542939 & .0670407 \\
\hline year_1997 & .0778934 & .0322136 & 2.42 & 0.017 & .0141073 & .1416795 \\
\hline year_1998 & .0347102 & .0363873 & 0.95 & 0.342 & -.0373403 & .1067606 \\
\hline year 1999 & .0249719 & .0367975 & 0.68 & 0.499 & -.0478909 & .0978347 \\
\hline year 2000 & .0941059 & .0406026 & 2.32 & 0.022 & .0137086 & .1745031 \\
\hline year_2001 & .035328 & .0349216 & 1.01 & 0.314 & -.0338203 & .1044763 \\
\hline year_2002 & .0508182 & .0382741 & 1.33 & 0.187 & -.0249682 & .1266047 \\
\hline year 2003 & .03714 & .0351861 & 1.06 & 0.293 & -.032532 & .106812 \\
\hline $\operatorname{may} 1631$ & .065168 & .013058 & 4.99 & 0.000 & .0393119 & .0910242 \\
\hline jun115 & .1385205 & .0153816 & 9.01 & 0.000 & .1080633 & .1689776 \\
\hline jun 1630 & .253638 & .021064 & 12.04 & 0.000 & .2119293 & .2953468 \\
\hline jul115 & .355915 & .0229292 & 15.52 & 0.000 & .3105128 & .4013171 \\
\hline jull631 & .4163466 & .0157949 & 26.36 & 0.000 & .385071 & .4476221 \\
\hline $\operatorname{aug} 115$ & .4347577 & .0186024 & 23.37 & 0.000 & .3979231 & .4715922 \\
\hline harlania & .0071553 & .01133 & 0.63 & 0.529 & -.0152791 & .0295898 \\
\hline gdd_harlania & -.0002341 & .0008936 & -0.26 & 0.794 & -.0020035 & .0015353 \\
\hline cp 2 harlan & -.0013018 & .0017644 & -0.74 & 0.462 & -.0047955 & .0021919 \\
\hline gdd2_harlan & $-1.11 e-06$ & $7.18 e-06$ & -0.16 & 0.877 & -.0000153 & .0000131 \\
\hline cpgdd_harlan & -.0000366 & .000219 & -0.17 & 0.868 & -.0004703 & .0003971 \\
\hline cons & .3220499 & .0310559 & 10.37 & 0.000 & .2605561 & .3835437 \\
\hline
\end{tabular}




\title{
Lamesa, TX
}

\author{
Linear regression
}

$\begin{array}{llr}\text { Number of obs } & & 153 \\ \text { F }(32,120) & 5.73 \\ \text { Prob F } & =0000 \\ \text { R-squared } & =0.6576 \\ \text { Root MSE } & = & .04742\end{array}$

\begin{tabular}{|c|c|c|c|c|c|c|}
\hline ndvi_lames $\sim \mathrm{x}$ & Coef. & $\begin{array}{l}\text { Robust HC3 } \\
\text { Std. Err. }\end{array}$ & t & $P>|t|$ & [95\% Conf. & Interval] \\
\hline year_1983 & -.0647132 & .0324342 & -2.00 & 0.048 & -.1289306 & -.0004959 \\
\hline year_1984 & -.0518028 & .0265306 & -1.95 & 0.053 & -.1043315 & .000726 \\
\hline year_1985 & .040959 & .0332348 & 1.23 & 0.220 & -.0248436 & .1067616 \\
\hline year_1986 & .0012476 & .0318445 & 0.04 & 0.969 & -.0618023 & .0642975 \\
\hline year_1987 & -.0041769 & .0355078 & -0.12 & 0.907 & -.0744799 & .066126 \\
\hline year_1988 & .0467098 & .0322429 & 1.45 & 0.150 & -.0171289 & .1105484 \\
\hline year_1989 & -.0630753 & .0273823 & -2.30 & 0.023 & -.1172903 & -.0088603 \\
\hline year_1990 & -.0350731 & .0332311 & -1.06 & 0.293 & -.1008684 & .0307221 \\
\hline year_1991 & -.0081645 & .0252632 & -0.32 & 0.747 & -.0581838 & .0418548 \\
\hline year-1992 & -.03945 & .0322151 & -1.22 & 0.223 & -.1032337 & .0243337 \\
\hline year_1993 & .0006648 & .0274264 & 0.02 & 0.981 & -.0536375 & .0549672 \\
\hline year 1994 & -.0326194 & .0310985 & -1.05 & 0.296 & -.0941922 & .0289534 \\
\hline year_1995 & -.0054003 & .0250366 & -0.22 & 0.830 & -.0549711 & .0441705 \\
\hline year_1996 & .0189786 & .0313236 & 0.61 & 0.546 & -.04304 & .0809972 \\
\hline year_1997 & .0868243 & .0404346 & 2.15 & 0.034 & .0067666 & .166882 \\
\hline year_1998 & -.0012898 & .0276141 & -0.05 & 0.963 & -.0559638 & .0533842 \\
\hline year_1999 & -.0035865 & .0331853 & -0.11 & 0.914 & -.0692911 & .0621181 \\
\hline year_2000 & .0361655 & .0352703 & 1.03 & 0.307 & -.0336673 & .1059983 \\
\hline year_2001 & -.0242203 & .0326803 & -0.74 & 0.460 & -.0889251 & .0404844 \\
\hline year_2002 & .0276534 & .0433087 & 0.64 & 0.524 & -.0580947 & .1134016 \\
\hline year_2003 & -.0283396 & .0335134 & -0.85 & 0.399 & -.0946938 & .0380145 \\
\hline $\operatorname{may} 1631$ & .0010506 & .0134609 & 0.08 & 0.938 & -.025601 & .0277023 \\
\hline jun115 & .0035763 & .0133086 & 0.27 & 0.789 & -.0227737 & .0299264 \\
\hline jun1630 & .0161765 & .0128504 & 1.26 & 0.211 & -.0092664 & .0416193 \\
\hline jul115 & .0464714 & .0150247 & 3.09 & 0.002 & .0167234 & .0762193 \\
\hline jul1631 & .0772585 & .0134597 & 5.74 & 0.000 & .0506093 & .1039077 \\
\hline aug115 & .1269956 & .0199971 & 6.35 & 0.000 & .0874028 & .1665884 \\
\hline cp_lamesatx & -.0116525 & .0116446 & -1.00 & 0.319 & -.034708 & .0114031 \\
\hline gdd_lamesatx & -.0026748 & .0231391 & -0.12 & 0.908 & -.0484886 & .043139 \\
\hline cр 2 2_lamesa & .0029565 & .0031275 & 0.95 & 0.346 & -.0032358 & .0091488 \\
\hline gdd2_lamesa & .0014639 & .0033438 & 0.44 & 0.662 & -.0051566 & .0080844 \\
\hline cpgdd_lamesa & -.0032679 & .0096334 & -0.34 & 0.735 & -.0223414 & .0158057 \\
\hline - cons & .2129291 & .026119 & 8.15 & 0.000 & .1612153 & .2646429 \\
\hline
\end{tabular}




\title{
Saluda, SC
}

\author{
Linear regression
}

$\begin{array}{llr}\text { Number of obs } & & 154 \\ \text { F }(32,121) & 1.86 \\ \text { Prob F } & =0087 \\ \text { R-squared } & =0.3270 \\ \text { Root MSE } & = & .03797\end{array}$

\begin{tabular}{|c|c|c|c|c|c|c|}
\hline ndvi_salud c & Coef. & $\begin{array}{c}\text { Robust } \mathrm{HC} 3 \\
\text { Std. Err. }\end{array}$ & $t$ & $P>|t|$ & [95\% Conf. & Interval] \\
\hline year_1983 & -.0133267 & .0305208 & -0.44 & 0.663 & -.0737507 & .0470973 \\
\hline year-1984 & -.0146859 & .0223718 & -0.66 & 0.513 & -.0589769 & .029605 \\
\hline year-1985 & -.0176807 & .0257296 & -0.69 & 0.493 & -.0686191 & .0332578 \\
\hline year_1986 & -.0059804 & .0216418 & -0.28 & 0.783 & -.0488261 & .0368653 \\
\hline year-1987 & .0313475 & .0233169 & 1.34 & 0.181 & -.0148144 & .0775093 \\
\hline year 1988 & .0122783 & .0201745 & 0.61 & 0.544 & -.0276624 & .0522189 \\
\hline year 1989 & .0158712 & .0225831 & 0.70 & 0.484 & -.0288379 & .0605803 \\
\hline year 1990 & -.0171496 & .0284829 & -0.60 & 0.548 & -.073539 & .0392398 \\
\hline year-1991 & .019074 & .0212967 & 0.90 & 0.372 & -.0230884 & .0612365 \\
\hline year 1992 & .0103377 & .0226208 & 0.46 & 0.648 & -.0344462 & .0551215 \\
\hline year 1993 & .0130383 & .024283 & 0.54 & 0.592 & -.0350363 & .0611129 \\
\hline year-1994 & -.0223628 & .02118 & -1.06 & 0.293 & -.0642942 & .0195687 \\
\hline year-1995 & -.0142158 & .0235032 & -0.60 & 0.546 & -.0607466 & .032315 \\
\hline year_1996 & -.0109989 & .0247652 & -0.44 & 0.658 & -.0600282 & .0380304 \\
\hline year_1997 & -.0058467 & .0214018 & -0.27 & 0.785 & -.0482172 & .0365238 \\
\hline year-1998 & -.003443 & .0282674 & -0.12 & 0.903 & -.0594057 & .0525198 \\
\hline year-1999 & -.0182898 & .0251755 & -0.73 & 0.469 & -.0681314 & .0315519 \\
\hline year_2000 & .0019528 & .0224424 & 0.09 & 0.931 & -.0424778 & .0463834 \\
\hline year 2001 & -.0063269 & .0266743 & -0.24 & 0.813 & -.0591356 & .0464818 \\
\hline year 2002 & .0062415 & .0285401 & 0.22 & 0.827 & -.0502612 & .0627442 \\
\hline year 2003 & .0161951 & .0233219 & 0.69 & 0.489 & -.0299768 & .062367 \\
\hline $\operatorname{may} 1631$ & -.0054063 & .0119593 & -0.45 & 0.652 & -.029083 & .0182703 \\
\hline jun115 & -.0122848 & .0101868 & -1.21 & 0.230 & -.0324522 & .0078827 \\
\hline jun 1630 & -.0240404 & .0124738 & -1.93 & 0.056 & -.0487355 & .0006547 \\
\hline jul115 & -.03439 & .0134866 & -2.55 & 0.012 & -.0610902 & -.0076897 \\
\hline jull631 & -.0416251 & .0125139 & -3.33 & 0.001 & -.0663997 & -.0168506 \\
\hline $\operatorname{aug} 115$ & -.0505575 & .0144319 & -3.50 & 0.001 & -.0791293 & -.0219858 \\
\hline saludasc & .0031002 & .0061113 & 0.51 & 0.613 & -.0089987 & .0151991 \\
\hline gdd_saludasc & .008905 & .2681629 & 0.03 & 0.974 & -.5219943 & .5398042 \\
\hline c $\bar{p} 2$ _saluda & -.0008605 & .0008776 & -0.98 & 0.329 & -.002598 & .000877 \\
\hline gdd2_saluda & -.0009544 & .0854286 & -0.01 & 0.991 & -.1700828 & .1681739 \\
\hline cpgdd_saluda & .0050412 & .0345609 & 0.15 & 0.884 & -.0633812 & .0734636 \\
\hline cons & .7379652 & .0221517 & 33.31 & 0.000 & .6941101 & .7818203 \\
\hline
\end{tabular}


Rock Rapids, IA

Linear regression

$\begin{array}{llr}\text { Number of obs } & = & 153 \\ \mathrm{~F}(30,122) & 72.65 \\ \text { Prob }>\text { F } & =.0000 \\ \text { R-squared } & =0.9335 \\ \text { Root MSE } & =.0524\end{array}$

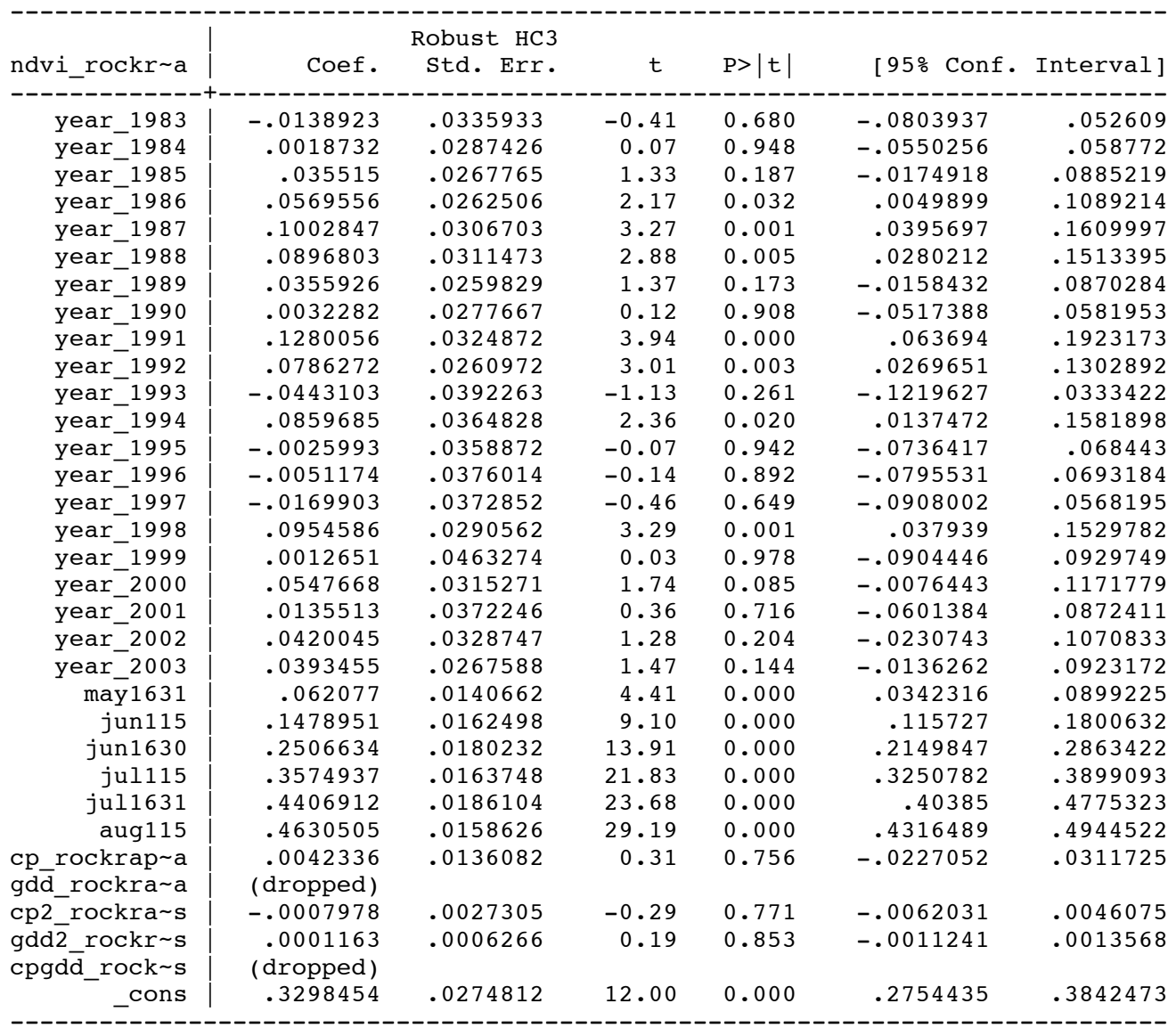


Osage, IA

Linear regression

$\begin{array}{llr}\text { Number of obs } & = & 154 \\ \mathrm{~F}(32, \text { 121) } & 31.19 \\ \text { Prob }>\text { F } & 0.0000 \\ \text { R-squared } & =0.8326 \\ \text { Root MSE } & = & .07938\end{array}$

\begin{tabular}{|c|c|c|c|c|c|c|}
\hline ndvi_osageia & Coef. & $\begin{array}{l}\text { Robust } \mathrm{HC} 3 \\
\text { Std. Err. }\end{array}$ & $t$ & $P>|t|$ & [95\% Conf. & Interval] \\
\hline year_1983 & -.0164564 & .0304734 & -0.54 & 0.590 & -.0767864 & .0438737 \\
\hline year_1984 & .0498406 & .0363174 & 1.37 & 0.172 & -.0220593 & .1217405 \\
\hline year_1985 & .057668 & .037081 & 1.56 & 0.123 & -.0157437 & .1310797 \\
\hline year_1986 & .0511312 & .034205 & 1.49 & 0.138 & -.0165866 & .1188489 \\
\hline year_1987 & .0804482 & .0452204 & 1.78 & 0.078 & -.0090776 & .1699739 \\
\hline year_1988 & .1032706 & .0399327 & 2.59 & 0.011 & .0242133 & .1823278 \\
\hline year_1989 & .0408183 & .0397694 & 1.03 & 0.307 & -.0379157 & .1195523 \\
\hline year_1990 & .0546896 & .0344535 & 1.59 & 0.115 & -.0135202 & .1228993 \\
\hline year_1991 & .058003 & .0328898 & 1.76 & 0.080 & -.007111 & .1231169 \\
\hline year_1992 & .0512332 & .0298257 & 1.72 & 0.088 & -.0078146 & .110281 \\
\hline year_1993 & -.0138394 & .0367382 & -0.38 & 0.707 & -.0865724 & .0588936 \\
\hline year_1994 & .0813703 & .0343629 & 2.37 & 0.019 & .0133399 & .1494007 \\
\hline year_1995 & .0611683 & .0372612 & 1.64 & 0.103 & -.0126 & .1349366 \\
\hline year_1996 & -.0917982 & .0907089 & -1.01 & 0.314 & -.2713804 & .0877839 \\
\hline year_1997 & .0106676 & .0441193 & 0.24 & 0.809 & -.0766782 & .0980133 \\
\hline year_1998 & .0252325 & .0339919 & 0.74 & 0.459 & -.0420633 & .0925284 \\
\hline year_1999 & -.1106733 & .0474019 & -2.33 & 0.021 & -.2045179 & -.0168288 \\
\hline year_2000 & -.0935542 & .0529115 & -1.77 & 0.080 & -.1983064 & .011198 \\
\hline year_2001 & -.0280956 & .0371694 & -0.76 & 0.451 & -.1016823 & .0454911 \\
\hline year_2002 & .0579556 & .0305697 & 1.90 & 0.060 & -.0025653 & .1184764 \\
\hline year_2003 & -.0478332 & .073356 & -0.65 & 0.516 & -.1930608 & .0973944 \\
\hline maȳ1631 & .0674751 & .0188541 & 3.58 & 0.000 & .0301484 & .1048018 \\
\hline jun115 & .1379693 & .0225097 & 6.13 & 0.000 & .0934055 & .1825332 \\
\hline jun 1630 & .2496278 & .0229949 & 10.86 & 0.000 & .2041034 & .2951522 \\
\hline jul115 & .350077 & .0237315 & 14.75 & 0.000 & .3030942 & .3970598 \\
\hline jull631 & .36856 & .0393416 & 9.37 & 0.000 & .2906729 & .446447 \\
\hline $\operatorname{aug} 115$ & .41341 & .0306637 & 13.48 & 0.000 & .352703 & .474117 \\
\hline cp_osageia & .0122178 & .0249823 & 0.49 & 0.626 & -.0372412 & .0616768 \\
\hline gdd_osageia & .0007108 & .001246 & 0.57 & 0.569 & -.001756 & .0031775 \\
\hline ср 2 osage & .0007253 & .0045143 & 0.16 & 0.873 & -.0082119 & .0096626 \\
\hline gdd2_osage & $-5.09 e-06$ & $9.62 e-06$ & -0.53 & 0.598 & -.0000241 & .000014 \\
\hline cpgdd_osage & -.0004949 & .0002327 & -2.13 & 0.035 & -.0009555 & -.0000343 \\
\hline cons & .3481433 & .0394637 & 8.82 & 0.000 & .2700145 & .4262721 \\
\hline
\end{tabular}


Allendale, SC

Linear regression

$\begin{array}{llr}\text { Number of obs } & & 150 \\ \mathrm{~F}(32,117) & = & 2.15 \\ \text { Prob }>\mathrm{F} & = & 0.0017 \\ \text { R-squared } & = & 0.3351 \\ \text { Root MSE } & = & .04489\end{array}$

\begin{tabular}{|c|c|c|c|c|c|c|}
\hline ndvi_allen c & Coef. & $\begin{array}{l}\text { Robust } \mathrm{HC} 3 \\
\text { Std. Err. }\end{array}$ & $t$ & $\mathrm{P}>|\mathrm{t}|$ & [95\% Conf. & Interval] \\
\hline- & --1 & & & & & --- \\
\hline year_1983 & .0377035 & .0288002 & 1.31 & 0.193 & -.0193339 & .0947409 \\
\hline year_1984 & .0151818 & .0314977 & 0.48 & 0.631 & -.0471977 & .0775613 \\
\hline year_1985 & .0069148 & .0366769 & 0.19 & 0.851 & -.0657218 & .0795514 \\
\hline year_1986 & -.0231008 & .0262304 & -0.88 & 0.380 & -.0750488 & .0288471 \\
\hline year_1987 & .0191538 & .0283644 & 0.68 & 0.501 & -.0370205 & .0753281 \\
\hline year_1988 & -.003618 & .0258504 & -0.14 & 0.889 & -.0548134 & .0475774 \\
\hline year_1989 & .0231153 & .0230725 & 1.00 & 0.318 & -.0225785 & .0688091 \\
\hline year_1990 & .0432666 & .0285907 & 1.51 & 0.133 & -.0133558 & .0998891 \\
\hline year_1991 & .0544657 & .0266061 & 2.05 & 0.043 & .0017738 & .1071576 \\
\hline year_1992 & .0437995 & .0232691 & 1.88 & 0.062 & -.0022839 & .0898828 \\
\hline year_1993 & -.0214545 & .0260782 & -0.82 & 0.412 & -.073101 & .0301921 \\
\hline year_1994 & -.0415796 & .0364549 & -1.14 & 0.256 & -.1137765 & .0306173 \\
\hline year_1995 & .0221335 & .0282509 & 0.78 & 0.435 & -.033816 & .078083 \\
\hline year_1996 & .0591521 & .0247581 & 2.39 & 0.018 & .01012 & .1081842 \\
\hline year_1997 & .0191817 & .0287888 & 0.67 & 0.507 & -.0378331 & .0761965 \\
\hline year_1998 & .0388658 & .0288853 & 1.35 & 0.181 & -.0183401 & .0960717 \\
\hline year_1999 & .0003478 & .0346925 & 0.01 & 0.992 & -.0683589 & .0690545 \\
\hline year_2000 & .0033286 & .0328328 & 0.10 & 0.919 & -.061695 & .0683523 \\
\hline year_2001 & .0470589 & .0286984 & 1.64 & 0.104 & -.0097769 & .1038946 \\
\hline year_2002 & .0509944 & .0422889 & 1.21 & 0.230 & -.0327566 & .1347454 \\
\hline year_2003 & .0243069 & .0312664 & 0.78 & 0.438 & -.0376146 & .0862284 \\
\hline $\operatorname{may} 1631$ & -.0099237 & .0140733 & -0.71 & 0.482 & -.0377952 & .0179478 \\
\hline jun115 & -.0131857 & .0152069 & -0.87 & 0.388 & -.0433022 & .0169307 \\
\hline jun 1630 & -.0132851 & .0136152 & -0.98 & 0.331 & -.0402492 & .0136791 \\
\hline jul115 & -.0016947 & .015873 & -0.11 & 0.915 & -.0331303 & .0297409 \\
\hline jul1631 & -.0128539 & .0182726 & -0.70 & 0.483 & -.0490419 & .0233341 \\
\hline aug115 & .0048848 & .0159478 & 0.31 & 0.760 & -.0266989 & .0364685 \\
\hline cp_allenda c & -.0092542 & .0077681 & -1.19 & 0.236 & -.0246385 & .00613 \\
\hline gdd_allend c & -.0265661 & .0120217 & -2.21 & 0.029 & -.0503744 & -.0027579 \\
\hline cp2_allend e & .001274 & .0011796 & 1.08 & 0.282 & -.0010621 & .0036101 \\
\hline gdd $\overline{2}$ allen e & .0022896 & .0009333 & 2.45 & 0.016 & .0004413 & .0041379 \\
\hline cpgdd_alle e & .0067721 & .0038107 & 1.78 & 0.078 & -.0007747 & .014319 \\
\hline - cons & .6253214 & .0253548 & 24.66 & 0.000 & .5751075 & .6755352 \\
\hline
\end{tabular}




\section{Pelion, SC}

Linear regression

$\begin{array}{llr}\text { Number of obs } & & 152 \\ \mathrm{~F}(32, \text { 119) } & 1.31 \\ \text { Prob }>\text { F } & =0.1475 \\ \text { R-squared } & =0.2878 \\ \text { Root MSE } & = & .05257\end{array}$

\begin{tabular}{|c|c|c|c|c|c|c|}
\hline ndvi_pelio c & Coef. & $\begin{array}{c}\text { Robust } \mathrm{HC} 3 \\
\text { Std. Err. }\end{array}$ & $t$ & $P>|t|$ & [ $95 \%$ Conf. & Interval] \\
\hline year_1983 & .0174782 & .028806 & 0.61 & 0.545 & -.0395605 & .0745169 \\
\hline year_1984 & .0569112 & .0262224 & 2.17 & 0.032 & .0049883 & .1088342 \\
\hline year_1985 & .0030455 & .0359633 & 0.08 & 0.933 & -.0681654 & .0742565 \\
\hline year_1986 & -.0287244 & .0258732 & -1.11 & 0.269 & -.0799559 & .0225072 \\
\hline year_1987 & .0137118 & .0265692 & 0.52 & 0.607 & -.0388979 & .0663214 \\
\hline year_1988 & .0237045 & .0338449 & 0.70 & 0.485 & -.0433119 & .0907208 \\
\hline year_1989 & .0207856 & .0252275 & 0.82 & 0.412 & -.0291674 & .0707385 \\
\hline year_1990 & .0391238 & .03228 & 1.21 & 0.228 & -.0247937 & .1030414 \\
\hline year_1991 & .0086384 & .0289854 & 0.30 & 0.766 & -.0487556 & .0660324 \\
\hline year_1992 & .0032081 & .0259884 & 0.12 & 0.902 & -.0482514 & .0546677 \\
\hline year_1993 & .0088595 & .0324147 & 0.27 & 0.785 & -.0553249 & .0730439 \\
\hline year_1994 & -.00338 & .0306049 & -0.11 & 0.912 & -.0639807 & .0572206 \\
\hline year_1995 & -.0363089 & .0363323 & -1.00 & 0.320 & -.1082506 & .0356327 \\
\hline year_1996 & .0433411 & .0282803 & 1.53 & 0.128 & -.0126566 & .0993389 \\
\hline year_1997 & .040136 & .0348596 & 1.15 & 0.252 & -.0288895 & .1091614 \\
\hline year_1998 & .0397993 & .033354 & 1.19 & 0.235 & -.026245 & .1058435 \\
\hline year_1999 & -.0161902 & .0576511 & -0.28 & 0.779 & -.1303451 & .0979647 \\
\hline year_2000 & .0328968 & .0296082 & 1.11 & 0.269 & -.0257304 & .091524 \\
\hline year_2001 & .0280634 & .0265045 & 1.06 & 0.292 & -.0244182 & .080545 \\
\hline year_2002 & .0503189 & .0375643 & 1.34 & 0.183 & -.0240621 & .1246999 \\
\hline year_2003 & .0311154 & .0256802 & 1.21 & 0.228 & -.019734 & .0819648 \\
\hline $\operatorname{may} 1631$ & -.0073731 & .0173353 & -0.43 & 0.671 & -.0416988 & .0269525 \\
\hline jun115 & -.0235228 & .0218173 & -1.08 & 0.283 & -.0667233 & .0196777 \\
\hline jun 1630 & -.0403938 & .02724 & -1.48 & 0.141 & -.0943317 & .0135442 \\
\hline jul115 & -.0342622 & .0283007 & -1.21 & 0.228 & -.0903004 & .021776 \\
\hline jul1631 & -.053627 & .0293381 & -1.83 & 0.070 & -.1117193 & .0044653 \\
\hline $\operatorname{aug} 115$ & -.0273682 & .0275049 & -1.00 & 0.322 & -.0818306 & .0270942 \\
\hline ?pelionsc & -.0024774 & .0130525 & -0.19 & 0.850 & -.0283227 & .0233679 \\
\hline gdd_pelionsc & -.0006508 & .0007334 & -0.89 & 0.377 & -.002103 & .0008014 \\
\hline cp2_pelion & -.0004042 & .001129 & -0.36 & 0.721 & -.0026398 & .0018314 \\
\hline gdd2_pelion & $4.12 e-06$ & $2.69 e-06$ & 1.53 & 0.129 & $-1.21 e-06$ & $9.45 e-06$ \\
\hline cpgdd_pelion & .0000633 & .0000882 & 0.72 & 0.475 & -.0001114 & .0002379 \\
\hline cons & .5713447 & .0396223 & 14.42 & 0.000 & .4928885 & .6498008 \\
\hline
\end{tabular}




\section{Dublin, GA}

Linear regression

$\begin{array}{llr}\text { Number of obs } & & 153 \\ \text { F }(32, \text { 120) } & =1.32 \\ \text { Prob F } & =0.1470 \\ \text { R-squared } & =0.3116 \\ \text { Root MSE } & = & .04107\end{array}$

\begin{tabular}{|c|c|c|c|c|c|c|}
\hline ndvi_dubli a & Coef. & $\begin{array}{c}\text { Robust HC3 } \\
\text { Std. Err. }\end{array}$ & $t$ & $P>|t|$ & [95\% Conf. & Interval] \\
\hline year 1983 & .0289745 & .0325073 & 0.89 & 0.375 & -.0353876 & .0933367 \\
\hline year 1984 & .0787168 & .0313205 & 2.51 & 0.013 & .0167044 & .1407291 \\
\hline year 1985 & .0605293 & .028331 & 2.14 & 0.035 & .004436 & .1166227 \\
\hline year 1986 & .0507824 & .0246908 & 2.06 & 0.042 & .0018964 & .0996684 \\
\hline year_1987 & .0877314 & .0303897 & 2.89 & 0.005 & .0275619 & .1479009 \\
\hline year 1988 & .0209702 & .0313666 & 0.67 & 0.505 & -.0411335 & .0830739 \\
\hline year 1989 & .0742932 & .0231785 & 3.21 & 0.002 & .0284014 & .1201851 \\
\hline year 1990 & .0531916 & .0212985 & 2.50 & 0.014 & .0110222 & .0953611 \\
\hline year 1991 & .0641644 & .0229702 & 2.79 & 0.006 & .018685 & .1096438 \\
\hline year 1992 & .0708557 & .0236809 & 2.99 & 0.003 & .0239691 & .1177422 \\
\hline year 1993 & .0797783 & .0267811 & 2.98 & 0.004 & .0267536 & .132803 \\
\hline year 1994 & .0658798 & .0227668 & 2.89 & 0.005 & .0208031 & .1109564 \\
\hline year 1995 & .0690353 & .0231058 & 2.99 & 0.003 & .0232873 & .1147832 \\
\hline year 1996 & .0607522 & .0258925 & 2.35 & 0.021 & .0094868 & .1120176 \\
\hline year-1997 & .0594048 & .0263079 & 2.26 & 0.026 & .007317 & .1114926 \\
\hline year 1998 & .0557818 & .0224136 & 2.49 & 0.014 & .0114043 & .1001593 \\
\hline year 1999 & .0098038 & .0266576 & 0.37 & 0.714 & -.0429763 & .062584 \\
\hline year 2000 & .0534614 & .0264946 & 2.02 & 0.046 & .001004 & .1059188 \\
\hline year 2001 & .0170087 & .0309648 & 0.55 & 0.584 & -.0442994 & .0783168 \\
\hline year_2002 & .0473914 & .0234915 & 2.02 & 0.046 & .0008798 & .093903 \\
\hline year 2003 & .0514777 & .0237309 & 2.17 & 0.032 & .0044921 & .0984633 \\
\hline may1631 & -.0096795 & .0114107 & -0.85 & 0.398 & -.032272 & .0129129 \\
\hline jun 115 & -.0207894 & .0132803 & -1.57 & 0.120 & -.0470834 & .0055046 \\
\hline jun 1630 & -.0114478 & .0106516 & -1.07 & 0.285 & -.0325372 & .0096416 \\
\hline jull15 & -.0112703 & .0126047 & -0.89 & 0.373 & -.0362266 & .0136861 \\
\hline jul1631 & -.0004774 & .0130368 & -0.04 & 0.971 & -.0262894 & .0253346 \\
\hline aug 115 & -.0046704 & .0153921 & -0.30 & 0.762 & -.0351457 & .0258048 \\
\hline cp dublinga & -.0065288 & .0086248 & -0.76 & 0.451 & -.0236053 & .0105478 \\
\hline gdd dublinga & .0012266 & .0023049 & 0.53 & 0.596 & -.003337 & .0057902 \\
\hline cp 2 dublin & .0000732 & .0016659 & 0.04 & 0.965 & -.0032252 & .0033716 \\
\hline gdd2_dublin & $-9.44 e-06$ & .0000742 & -0.13 & 0.899 & -.0001563 & .0001374 \\
\hline cpgdd_dublin & -.0006931 & .0006593 & -1.05 & 0.295 & -.0019986 & .0006123 \\
\hline cons & .5826568 & .0210405 & 27.69 & 0.000 & .540998 & .6243156 \\
\hline
\end{tabular}




\section{Siloam, GA}

Linear regression

$\begin{array}{llr}\text { Number of obs } & = & 150 \\ \mathrm{~F}(32, \text { 117) } & = & 2.06 \\ \text { Prob }>\text { F } & =0029 \\ \text { R-squared } & =0.3752 \\ \text { Root MSE } & = & .05103\end{array}$

\begin{tabular}{|c|c|c|c|c|c|c|}
\hline ndvi_siloa a & Coef. & $\begin{array}{l}\text { Robust } \mathrm{HC} 3 \\
\text { Std. Err. }\end{array}$ & $t$ & $P>|t|$ & [95\% & Int \\
\hline year_1983 & -.0181615 & .0303225 & -0.60 & 0.550 & -.0782136 & .0418906 \\
\hline year_1984 & .0091696 & .027476 & 0.33 & 0.739 & -.0452452 & .0635844 \\
\hline year_1985 & .0304348 & .0301616 & 1.01 & 0.315 & -.0292987 & .0901683 \\
\hline year_1986 & -.0331455 & .0326907 & -1.01 & 0.313 & -.0978878 & .0315968 \\
\hline year_1987 & .0328917 & .0254032 & 1.29 & 0.198 & -.017418 & .0832014 \\
\hline year_1988 & -.0498088 & .0493309 & -1.01 & 0.315 & -.147506 & .0478884 \\
\hline year_1989 & .0330712 & .0348717 & 0.95 & 0.345 & -.0359903 & .1021327 \\
\hline year_1990 & .002065 & .0296443 & 0.07 & 0.945 & -.0566439 & .0607739 \\
\hline year_1991 & -.0105017 & .0364102 & -0.29 & 0.774 & -.0826102 & .0616067 \\
\hline year_1992 & .0266743 & .0327924 & 0.81 & 0.418 & -.0382694 & .0916179 \\
\hline year_1993 & .0022566 & .0281284 & 0.08 & 0.936 & -.0534503 & .0579635 \\
\hline year_1994 & -.0410583 & .0307072 & -1.34 & 0.184 & -.1018724 & .0197558 \\
\hline year_1995 & .0262753 & .0254434 & 1.03 & 0.304 & -.0241141 & .0766646 \\
\hline year_1996 & .0408565 & .02721 & 1.50 & 0.136 & -.0130314 & .0947444 \\
\hline year_1997 & .0501992 & .0252326 & 1.99 & 0.049 & .0002273 & .1001711 \\
\hline year_1998 & .0409059 & .0292174 & 1.40 & 0.164 & -.0169578 & .0987695 \\
\hline year_1999 & .0123601 & .0322683 & 0.38 & 0.702 & -.0515455 & .0762658 \\
\hline year_2000 & .0020515 & .0263229 & 0.08 & 0.938 & -.0500797 & .0541827 \\
\hline year_2001 & .0121016 & .028393 & 0.43 & 0.671 & -.0441293 & .0683325 \\
\hline year_2002 & .0336243 & .0268402 & 1.25 & 0.213 & -.0195314 & .08678 \\
\hline year_2003 & .0067823 & .0753085 & 0.09 & 0.928 & -.1423623 & .1559268 \\
\hline $\operatorname{may} 1631$ & -.0060882 & .0174725 & -0.35 & 0.728 & -.0406915 & .0285151 \\
\hline jun115 & -.0134723 & .0144736 & -0.93 & 0.354 & -.0421365 & .0151918 \\
\hline jun 1630 & -.0301852 & .0193738 & -1.56 & 0.122 & -.068554 & .0081835 \\
\hline jul115 & -.0418718 & .017829 & -2.35 & 0.021 & -.0771812 & -.0065624 \\
\hline jul1631 & -.0526073 & .0171672 & -3.06 & 0.003 & -.086606 & -.0186085 \\
\hline $\operatorname{aug} 115$ & -.0506844 & .0201352 & -2.52 & 0.013 & -.0905612 & -.0108076 \\
\hline siloamga & -.0078895 & .0114937 & -0.69 & 0.494 & -.0306521 & .0148731 \\
\hline gdd_siloamga & .0021886 & .0013844 & 1.58 & 0.117 & -.0005531 & .0049302 \\
\hline c $\bar{p} 2$ _siloam & .0003326 & .0022777 & 0.15 & 0.884 & -.0041781 & .0048434 \\
\hline gdd2_siloam & -.0000113 & $9.40 e-06$ & -1.21 & 0.230 & -.00003 & $7.28 e-06$ \\
\hline gdd_siloam & -.000287 & .0004265 & -0.67 & 0.502 & -.0011318 & .0005577 \\
\hline _cons & .7285777 & .0245159 & 29.72 & 0.000 & .6800253 & .7771301 \\
\hline
\end{tabular}




\section{Talbottan, GA}

Linear regression

$\begin{array}{llr}\text { Number of obs } & = & 149 \\ \mathrm{~F}(32, \quad 116) & = & 3.67 \\ \text { Prob }>\text { F } & = & 0.0000 \\ \text { R-squared } & =0.4928 \\ \text { Root MSE } & = & .03956\end{array}$

\begin{tabular}{|c|c|c|c|c|c|c|}
\hline ndvi_talbo a & Coef. & $\begin{array}{l}\text { Robust } \mathrm{HC} 3 \\
\text { Std. Err. }\end{array}$ & $t$ & $P>|t|$ & [95\% Conf. & Inte \\
\hline year_1983 & .0302649 & .0286938 & 1.05 & 0.294 & -.0265668 & .0870966 \\
\hline year_1984 & .0473669 & .0317344 & 1.49 & 0.138 & -.015487 & .1102208 \\
\hline year_1985 & .0198394 & .0260493 & 0.76 & 0.448 & -.0317545 & .0714333 \\
\hline year_1986 & -.0067956 & .0304178 & -0.22 & 0.824 & -.0670419 & .0534507 \\
\hline year_1987 & .0372574 & .0324249 & 1.15 & 0.253 & -.0269641 & .101479 \\
\hline year_1988 & -.0027883 & .0339703 & -0.08 & 0.935 & -.0700708 & .0644941 \\
\hline year_1989 & .0571335 & .0312748 & 1.83 & 0.070 & -.0048102 & .1190772 \\
\hline year_1990 & .0386001 & .0273944 & 1.41 & 0.161 & -.0156579 & .0928582 \\
\hline year_1991 & .0288123 & .0298876 & 0.96 & 0.337 & -.0303838 & .0880083 \\
\hline year_1992 & .0363252 & .0282008 & 1.29 & 0.200 & -.0195301 & .0921805 \\
\hline year_1993 & .0329307 & .026671 & 1.23 & 0.219 & -.0198945 & .0857559 \\
\hline year_1994 & -.0070901 & .0325205 & -0.22 & 0.828 & -.071501 & .0573208 \\
\hline year_1995 & .0324268 & .033154 & 0.98 & 0.330 & -.0332389 & .0980925 \\
\hline year_1996 & .0424042 & .0319374 & 1.33 & 0.187 & -.0208518 & .1056603 \\
\hline year_1997 & .0161714 & .0299808 & 0.54 & 0.591 & -.0432094 & .0755523 \\
\hline year_1998 & .0499314 & .0293432 & 1.70 & 0.092 & -.0081866 & .1080493 \\
\hline year_1999 & .0211974 & .0355069 & 0.60 & 0.552 & -.0491284 & .0915233 \\
\hline year_2000 & .0468102 & .0274304 & 1.71 & 0.091 & -.0075192 & .1011397 \\
\hline year_2001 & .0329025 & .0314585 & 1.05 & 0.298 & -.0294051 & .09521 \\
\hline year_2002 & .0603158 & .0387041 & 1.56 & 0.122 & -.0163425 & .1369742 \\
\hline year_2003 & .0538615 & .0355701 & 1.51 & 0.133 & -.0165897 & .1243126 \\
\hline $\operatorname{may} 1631$ & .0004039 & .0109333 & 0.04 & 0.971 & -.0212508 & .0220587 \\
\hline jun115 & -.0050624 & .0122866 & -0.41 & 0.681 & -.0293975 & .0192727 \\
\hline jun 1630 & -.0208266 & .0152005 & -1.37 & 0.173 & -.0509331 & .0092798 \\
\hline jul115 & -.0414037 & .0142316 & -2.91 & 0.004 & -.0695913 & -.0132162 \\
\hline jul1631 & -.0639083 & .0128931 & -4.96 & 0.000 & -.0894447 & -.038372 \\
\hline $\operatorname{aug} 115$ & -.0611223 & .0143987 & -4.24 & 0.000 & -.0896406 & -.0326039 \\
\hline albott a & -.0066123 & .0063538 & -1.04 & 0.300 & -.0191967 & .0059721 \\
\hline gdd_talbot a & .0004375 & .0004626 & 0.95 & 0.346 & -.0004787 & .0013537 \\
\hline cp2_talbot n & .0007366 & .0006805 & 1.08 & 0.281 & -.0006113 & .0020845 \\
\hline gdd 2 _talbo n & $-9.14 e-07$ & $2.68 e-06$ & -0.34 & 0.734 & $-6.23 e-06$ & $4.40 e-06$ \\
\hline pgdd__talb n & -.000135 & .0001014 & -1.33 & 0.185 & -.0003358 & .0000657 \\
\hline _cons & .7482175 & .0259556 & 28.83 & 0.000 & .6968093 & .7996258 \\
\hline
\end{tabular}




\section{Yazoo City, MS}

Linear regression

Number of obs =

$\mathrm{F}(32,121)=$

Prob > F

R-squared

Root MSE
154

253.20

$=0.0000$

$=0.9896$

$=.00952$

\begin{tabular}{|c|c|c|c|c|c|c|}
\hline ndvi_yazooms & Coef. & $\begin{array}{l}\text { Robust HC } 3 \\
\text { Std. Err. }\end{array}$ & t & $P>|t|$ & {$[95 \%$ Conf. } & Interval] \\
\hline \multicolumn{7}{|c|}{ 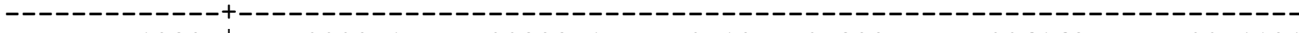 } \\
\hline year_1983 & -.0003717 & .0029251 & -0.13 & 0.899 & -.0061627 & .0054194 \\
\hline year_1984 & -.0013491 & .0080752 & -0.17 & 0.868 & -.017336 & .0146379 \\
\hline year_1985 & -.0005365 & .0030707 & -0.17 & 0.862 & -.0066158 & .0055429 \\
\hline year_1986 & .0052959 & .0035429 & 1.49 & 0.138 & -.0017182 & .0123101 \\
\hline year_1987 & -.0026026 & .0045615 & -0.57 & 0.569 & -.0116332 & .006428 \\
\hline year_1988 & -.0044786 & .0048031 & -0.93 & 0.353 & -.0139876 & .0050305 \\
\hline year_1989 & -.0062363 & .0073802 & -0.84 & 0.400 & -.0208473 & .0083748 \\
\hline year_1990 & .0034961 & .0026485 & 1.32 & 0.189 & -.0017473 & .0087394 \\
\hline year_1991 & -.0107852 & .0104111 & -1.04 & 0.302 & -.0313967 & .0098262 \\
\hline year_1992 & .0011311 & .0050033 & 0.23 & 0.822 & -.0087742 & .0110363 \\
\hline year_1993 & .0012975 & .0031179 & 0.42 & 0.678 & -.0048753 & .0074703 \\
\hline year_1994 & -.0039847 & .0043487 & -0.92 & 0.361 & -.012594 & .0046247 \\
\hline year_1995 & .0032002 & .0033178 & 0.96 & 0.337 & -.0033684 & .0097687 \\
\hline year_1996 & -.0077594 & .0031754 & -2.44 & 0.016 & -.0140459 & -.0014728 \\
\hline year_1997 & .0029369 & .0035451 & 0.83 & 0.409 & -.0040816 & .0099554 \\
\hline year_1998 & .0028569 & .0028013 & 1.02 & 0.310 & -.0026891 & .0084029 \\
\hline year_1999 & .0012285 & .0058298 & 0.21 & 0.833 & -.0103131 & .01277 \\
\hline year_2000 & .0020908 & .0024621 & 0.85 & 0.397 & -.0027836 & .0069652 \\
\hline year_2001 & .000558 & .0027438 & 0.20 & 0.839 & -.004874 & .00599 \\
\hline year_2002 & .003831 & .0027346 & 1.40 & 0.164 & -.0015828 & .0092448 \\
\hline year_2003 & .0044422 & .0028898 & 1.54 & 0.127 & -.0012788 & .0101633 \\
\hline $\operatorname{may} 1631$ & -.0094498 & .0046087 & -2.05 & 0.042 & -.0185739 & -.0003256 \\
\hline jun115 & -.0041086 & .0024825 & -1.66 & 0.101 & -.0090234 & .0008062 \\
\hline jun 1630 & -.0012872 & .0029808 & -0.43 & 0.667 & -.0071884 & .004614 \\
\hline jul115 & .0003403 & .0023105 & 0.15 & 0.883 & -.0042339 & .0049145 \\
\hline jul1631 & .0016586 & .0024858 & 0.67 & 0.506 & -.0032627 & .00658 \\
\hline $\operatorname{aug} 115$ & .001455 & .0020109 & 0.72 & 0.471 & -.0025261 & .005436 \\
\hline cp_yazooms & .0001249 & .0005316 & 0.23 & 0.815 & -.0009275 & .0011773 \\
\hline gdd_yazooms & .0026336 & .0061863 & 0.43 & 0.671 & -.0096138 & .014881 \\
\hline cp2_yazooms & .7745471 & .0287666 & 26.93 & 0.000 & .717596 & .8314981 \\
\hline gdd2_yazooms & $1.38 e-06$ & .000017 & 0.08 & 0.935 & -.0000323 & .0000351 \\
\hline cpgdd_yazo s & -.0038551 & .0082394 & -0.47 & 0.641 & -.0201671 & .0124569 \\
\hline cons & .316214 & .0159061 & 19.88 & 0.000 & .2847236 & .3477044 \\
\hline
\end{tabular}




\section{Batesville, MS}

Linear regression

Number of obs $=$

$\mathrm{F}(20,121)=$

Prob > F

R-squared

$=$
$=$

Root MSE

\begin{tabular}{|c|c|c|c|c|c|c|}
\hline ndvi_bates s & Coef. & $\begin{array}{l}\text { Robust } \mathrm{HC} 3 \\
\text { Std. Err. }\end{array}$ & $t$ & $P>|t|$ & [ $95 \%$ Conf. & Interval] \\
\hline year_1983 & .0004706 & .0031245 & 0.15 & 0.881 & -.0057152 & .0066563 \\
\hline year_1984 & -.0005206 & .0031191 & -0.17 & 0.868 & -.0066958 & .0056545 \\
\hline year_1985 & .0004626 & .0029194 & 0.16 & 0.874 & -.0053171 & .0062422 \\
\hline year_1986 & .0001382 & .0030011 & 0.05 & 0.963 & -.0058032 & .0060795 \\
\hline year_1987 & .0013427 & .006977 & 0.19 & 0.848 & -.0124701 & .0151555 \\
\hline year_1988 & -.0008812 & .0035263 & -0.25 & 0.803 & -.0078626 & .0061001 \\
\hline year_1989 & .000076 & .004701 & 0.02 & 0.987 & -.0092309 & .0093828 \\
\hline year_1990 & .0010678 & .0039504 & 0.27 & 0.787 & -.0067531 & .0088888 \\
\hline year_1991 & -.0002703 & .0026335 & -0.10 & 0.918 & -.005484 & .0049434 \\
\hline year_1992 & -.0014491 & .0051739 & -0.28 & 0.780 & -.0116922 & .008794 \\
\hline year_1993 & .0009648 & .0038402 & 0.25 & 0.802 & -.0066379 & .0085676 \\
\hline year_1994 & -.0010404 & .0042483 & -0.24 & 0.807 & -.009451 & .0073702 \\
\hline year_1995 & -.0017028 & .0058069 & -0.29 & 0.770 & -.0131991 & .0097934 \\
\hline year_1996 & -.0019051 & .0061775 & -0.31 & 0.758 & -.0141352 & .010325 \\
\hline year_1997 & -.0006085 & .0032298 & -0.19 & 0.851 & -.0070028 & .0057859 \\
\hline year_1998 & -.0010325 & .004092 & -0.25 & 0.801 & -.0091337 & .0070687 \\
\hline year_1999 & -.0176013 & .0508364 & -0.35 & 0.730 & -.1182454 & .0830428 \\
\hline year_2000 & -.0001124 & .0030158 & -0.04 & 0.970 & -.0060829 & .0058582 \\
\hline year_2001 & .0020182 & .0062261 & 0.32 & 0.746 & -.0103079 & .0143444 \\
\hline year_2002 & -.0015994 & .0054697 & -0.29 & 0.770 & -.0124281 & .0092293 \\
\hline year_2003 & -.0001954 & .0024546 & -0.08 & 0.937 & -.0050549 & .004664 \\
\hline maȳ1631 & -.0038282 & .0111382 & -0.34 & 0.732 & -.0258791 & .0182227 \\
\hline jun 115 & -.002187 & .0063443 & -0.34 & 0.731 & -.0147472 & .0103733 \\
\hline jun 1630 & -.0011698 & .0035954 & -0.33 & 0.745 & -.0082878 & .0059481 \\
\hline jul115 & .0002323 & .0016562 & 0.14 & 0.889 & -.0030466 & .0035112 \\
\hline jul1631 & -.0015707 & .0047605 & -0.33 & 0.742 & -.0109954 & .0078539 \\
\hline $\operatorname{aug} 115$ & -.0026228 & .0076125 & -0.34 & 0.731 & -.0176938 & .0124482 \\
\hline tesvi s & .000434 & .00128 & 0.34 & 0.735 & -.0021 & .0029681 \\
\hline oatesv $\sim \mathrm{s}$ & (dropped) & & & & & \\
\hline cp2_batesv s & .8311767 & 2.360074 & 0.35 & 0.725 & -3.841212 & 5.503565 \\
\hline dd2_bates s & .0003854 & 22035.62 & 0.00 & 1.000 & -43625.32 & 43625.32 \\
\hline $\bar{d}$ _bate $s$ & -.0054431 & 283933.8 & -0.00 & 1.000 & -562121.9 & 562121.9 \\
\hline Cons & .2985626 & .8477591 & 0.35 & 0.725 & -1.3798 & 1.976925 \\
\hline
\end{tabular}


Billings, MO

Linear regression

Number of obs =

154

$\mathrm{F}(31,122)=1808.46$

Prob > F $=0.0000$

R-squared $=0.9987$

Root MSE $=.00201$

\begin{tabular}{|c|c|c|c|c|c|c|}
\hline ndvi_billi o & Coef. & $\begin{array}{l}\text { Robust HC3 } \\
\text { Std. Err. }\end{array}$ & $t$ & $P>|t|$ & {$[95 \%$ Con $]$} & Int \\
\hline year_1983 & -.0019432 & .0010638 & -1.83 & 0.070 & -.0040492 & .0001628 \\
\hline year_1984 & -.0025031 & .0014764 & -1.70 & 0.093 & -.0054258 & .0004196 \\
\hline year_1985 & -.0020005 & .0008891 & -2.25 & 0.026 & -.0037606 & -.0002403 \\
\hline year_1986 & -.0014116 & .0012702 & -1.11 & 0.269 & -.0039262 & .001103 \\
\hline year_1987 & -.0007264 & .0009949 & -0.73 & 0.467 & -.0026959 & .0012431 \\
\hline year_1988 & -.0022191 & .0012378 & -1.79 & 0.075 & -.0046694 & .0002312 \\
\hline year_1989 & -.0008131 & .0008957 & -0.91 & 0.366 & -.0025862 & .0009599 \\
\hline year_1990 & -.0011713 & .0014568 & -0.80 & 0.423 & -.0040552 & .0017127 \\
\hline year_1991 & -.0014836 & .0011489 & -1.29 & 0.199 & -.0037579 & .0007907 \\
\hline year_1992 & -.0012675 & .0008469 & -1.50 & 0.137 & -.002944 & .000409 \\
\hline year_1993 & -.0025893 & .0013696 & -1.89 & 0.061 & -.0053005 & .0001219 \\
\hline year_1994 & -.0003844 & .0005572 & -0.69 & 0.492 & -.0014875 & .0007188 \\
\hline year_1995 & -.0011664 & .0009834 & -1.19 & 0.238 & -.003113 & .0007803 \\
\hline year_1996 & -.0007567 & .0007264 & -1.04 & 0.300 & -.0021947 & .0006814 \\
\hline year_1997 & -.0003687 & .0007357 & -0.50 & 0.617 & -.001825 & .0010877 \\
\hline year_1998 & -.0006112 & .0005892 & -1.04 & 0.302 & -.0017776 & .0005553 \\
\hline year_1999 & -.000318 & .000933 & -0.34 & 0.734 & -.002165 & .001529 \\
\hline year_2000 & -.0010512 & .0009069 & -1.16 & 0.249 & -.0028465 & .000744 \\
\hline year_2001 & -.0002649 & .0007119 & -0.37 & 0.710 & -.0016741 & .0011443 \\
\hline year_2002 & -.0017647 & .0009611 & -1.84 & 0.069 & -.0036673 & .0001379 \\
\hline year_2003 & .0001853 & .0005506 & 0.34 & 0.737 & -.0009047 & .0012752 \\
\hline $\operatorname{may} 1631$ & -.0012335 & .0007642 & -1.61 & 0.109 & -.0027463 & .0002794 \\
\hline jun 115 & -.0003436 & .0005839 & -0.59 & 0.557 & -.0014995 & .0008123 \\
\hline jun 1630 & -.0003972 & .0006293 & -0.63 & 0.529 & -.0016429 & .0008484 \\
\hline jul115 & -.0006114 & .0005617 & -1.09 & 0.279 & -.0017233 & .0005005 \\
\hline jul1631 & -.0005066 & .0006725 & -0.75 & 0.453 & -.0018378 & .0008246 \\
\hline $\operatorname{aug} 115$ & -.0005125 & .000634 & -0.81 & 0.420 & -.0017676 & .0007427 \\
\hline billing o & -.0000454 & .0001313 & -0.35 & 0.730 & -.0003054 & .0002146 \\
\hline gdd_billin o & -.0000636 & .0263038 & -0.00 & 0.998 & -.0521346 & .0520073 \\
\hline cp2_billin o & .7172349 & .0047265 & 151.75 & 0.000 & .7078783 & .7265916 \\
\hline $\begin{array}{l}\text { gdd } \overline{2} \text { billi o } \\
\text { cpgdd bill o }\end{array}$ & $\begin{array}{r}.0000447 \\
\text { (dropped) }\end{array}$ & .0052677 & 0.01 & 0.993 & -.0103833 & .0104727 \\
\hline cons & .348479 & .0024416 & 142.73 & 0.000 & .3436456 & .3533124 \\
\hline
\end{tabular}


Whatley, AL

Linear regression

Number of obs $=$

154

$\mathrm{F}(32,121)=1074.08$

Prob > F $=0.0000$

R-squared $\quad=0.9960$

Root MSE $=.00364$

\begin{tabular}{|c|c|c|c|c|c|c|}
\hline \multirow{2}{*}{ ndvi_whatl 1 } & \multicolumn{3}{|c|}{ Robust $\mathrm{HC} 3$} & \multirow[b]{2}{*}{$P>|t|$} & \multirow[b]{2}{*}{ [95\% Conf. } & \multirow[b]{2}{*}{ Interval } \\
\hline & Coef. & Std. Err. & $t$ & & & \\
\hline year 1983 & -.0045009 & .0043951 & -1.02 & 0.308 & -.0132021 & .0042003 \\
\hline year_1984 & -.0013929 & .0019453 & -0.72 & 0.475 & -.0052442 & .0024583 \\
\hline year_1985 & -.0027174 & .0025095 & -1.08 & 0.281 & -.0076856 & .0022508 \\
\hline year_1986 & .0014459 & .0017035 & 0.85 & 0.398 & -.0019267 & .0048185 \\
\hline year_1987 & .0004471 & .0018343 & 0.24 & 0.808 & -.0031843 & .0040785 \\
\hline year_1988 & -.0024634 & .0028168 & -0.87 & 0.384 & -.0080399 & .0031132 \\
\hline year 1989 & -.0004438 & .0016743 & -0.27 & 0.791 & -.0037586 & .0028709 \\
\hline year_1990 & -.0014201 & .0018396 & -0.77 & 0.442 & -.005062 & .0022218 \\
\hline year_1991 & -.0005895 & .0021331 & -0.28 & 0.783 & -.0048126 & .0036336 \\
\hline year 1992 & .0003683 & .0013551 & 0.27 & 0.786 & -.0023145 & .0030511 \\
\hline year 1993 & -.0009189 & .0013692 & -0.67 & 0.503 & -.0036295 & .0017917 \\
\hline year_1994 & .0005559 & .001426 & 0.39 & 0.697 & -.0022672 & .0033791 \\
\hline year_1995 & -.0014367 & .0014291 & -1.01 & 0.317 & -.0042659 & .0013925 \\
\hline year_1996 & -.0009747 & .0018334 & -0.53 & 0.596 & -.0046043 & .002655 \\
\hline year_1997 & -.0004654 & .0015507 & -0.30 & 0.765 & -.0035355 & .0026047 \\
\hline year_1998 & .000234 & .0012811 & 0.18 & 0.855 & -.0023023 & .0027703 \\
\hline year_1999 & .0009066 & .0014281 & 0.63 & 0.527 & -.0019208 & .0037339 \\
\hline year_2000 & .0000539 & .0011806 & 0.05 & 0.964 & -.0022834 & .0023912 \\
\hline year_2001 & -.0006779 & .0018172 & -0.37 & 0.710 & -.0042755 & .0029197 \\
\hline year_2002 & -.0006464 & .0014549 & -0.44 & 0.658 & -.0035267 & .0022339 \\
\hline year_2003 & .0004835 & .0010169 & 0.48 & 0.635 & -.0015297 & .0024967 \\
\hline $\operatorname{may} 1631$ & -.0015639 & .00113 & -1.38 & 0.169 & -.003801 & .0006733 \\
\hline jun115 & .0005477 & .0011138 & 0.49 & 0.624 & -.0016574 & .0027529 \\
\hline jun 1630 & -.0008722 & .0011576 & -0.75 & 0.453 & -.003164 & .0014196 \\
\hline jul115 & .0010431 & .0013097 & 0.80 & 0.427 & -.0015498 & .0036361 \\
\hline jul1631 & .0017734 & .0015862 & 1.12 & 0.266 & -.0013668 & .0049136 \\
\hline $\operatorname{aug} 115$ & .0013923 & .0016429 & 0.85 & 0.398 & -.0018602 & .0046447 \\
\hline whatleyal & .0001589 & .0001073 & 1.48 & 0.141 & -.0000536 & .0003714 \\
\hline dd__whatle 1 & .0002879 & .000419 & 0.69 & 0.493 & -.0005415 & .0011174 \\
\hline cp2_whatle 1 & .6925994 & .0230501 & 30.05 & 0.000 & .6469656 & .7382332 \\
\hline gdd $\overline{2}$ _whatl 1 & $-7.15 e-11$ & $2.99 e-07$ & -0.00 & 1.000 & $-5.92 e-07$ & $5.92 e-07$ \\
\hline gdd̄_what 1 & -.000375 & .0005285 & -0.71 & 0.479 & -.0014212 & .0006712 \\
\hline cons & .3578705 & .0138615 & 25.82 & 0.000 & .330428 & .3853129 \\
\hline
\end{tabular}




\section{Geneva, AL}

Linear regression

$\begin{array}{llr}\text { Number of obs } & = & 154 \\ \mathrm{~F}(32, \text { 121) } & = & 4.21 \\ \text { Prob }>\text { F } & =.0000 \\ \text { R-squared } & =0.4072 \\ \text { Root MSE } & = & .03865\end{array}$

\begin{tabular}{|c|c|c|c|c|c|c|}
\hline ndvi_geneva & Coef. & $\begin{array}{c}\text { Robust } \mathrm{HC} 3 \\
\text { Std. Err. }\end{array}$ & $t$ & $P>|t|$ & [ $95 \%$ Conf. & Interval] \\
\hline year_1983 & .0168221 & .0244037 & 0.69 & 0.492 & -.0314914 & .0651357 \\
\hline year 1984 & .0552678 & .0286398 & 1.93 & 0.056 & -.0014323 & .1119678 \\
\hline year 1985 & .0320217 & .0221221 & 1.45 & 0.150 & -.0117748 & .0758182 \\
\hline year 1986 & -.0136262 & .0327487 & -0.42 & 0.678 & -.0784609 & .0512084 \\
\hline year_1987 & .0480268 & .0262717 & 1.83 & 0.070 & -.0039849 & .1000385 \\
\hline year 1988 & .0037405 & .0400368 & 0.09 & 0.926 & -.0755228 & .0830039 \\
\hline year 1989 & .0319786 & .0236434 & 1.35 & 0.179 & -.0148297 & .0787868 \\
\hline year_1990 & .0679395 & .0242506 & 2.80 & 0.006 & .0199291 & .1159499 \\
\hline year_1991 & .0221802 & .0257313 & 0.86 & 0.390 & -.0287618 & .0731222 \\
\hline year 1992 & .0181951 & .0278175 & 0.65 & 0.514 & -.036877 & .0732671 \\
\hline year 1993 & .0600016 & .028043 & 2.14 & 0.034 & .0044831 & .1155201 \\
\hline year_1994 & -.0193104 & .0291236 & -0.66 & 0.509 & -.0769681 & .0383474 \\
\hline year_1995 & .0788524 & .024731 & 3.19 & 0.002 & .0298908 & .1278139 \\
\hline year_1996 & .0663852 & .0234738 & 2.83 & 0.005 & .0199126 & .1128579 \\
\hline year_1997 & .0422652 & .0245504 & 1.72 & 0.088 & -.0063388 & .0908692 \\
\hline year_1998 & .0315957 & .0231037 & 1.37 & 0.174 & -.0141441 & .0773355 \\
\hline year_1999 & .0379132 & .0293952 & 1.29 & 0.200 & -.0202823 & .0961087 \\
\hline year_2000 & .0062709 & .0263491 & 0.24 & 0.812 & -.045894 & .0584359 \\
\hline year 2001 & .0111018 & .0232526 & 0.48 & 0.634 & -.0349329 & .0571366 \\
\hline year 2002 & .0529343 & .023113 & 2.29 & 0.024 & .007176 & .0986926 \\
\hline year 2003 & .0364944 & .0223548 & 1.63 & 0.105 & -.0077627 & .0807516 \\
\hline $\operatorname{may} 1631$ & -.005704 & .0114569 & -0.50 & 0.619 & -.028386 & .0169781 \\
\hline jun115 & -.0028594 & .0107121 & -0.27 & 0.790 & -.0240669 & .0183481 \\
\hline jun 1630 & -.0182569 & .0154903 & -1.18 & 0.241 & -.0489241 & .0124103 \\
\hline jul115 & -.0121542 & .0111584 & -1.09 & 0.278 & -.0342451 & .0099368 \\
\hline jul1631 & -.009523 & .0125356 & -0.76 & 0.449 & -.0343405 & .0152945 \\
\hline $\operatorname{aug} 115$ & .0065086 & .0125719 & 0.52 & 0.606 & -.0183808 & .031398 \\
\hline genevaal & .0036268 & .0039313 & 0.92 & 0.358 & -.0041562 & .0114098 \\
\hline gdd_genevaal & .0002806 & .0020007 & 0.14 & 0.889 & -.0036803 & .0042415 \\
\hline cp2 genevaal & -.0004919 & .0003321 & -1.48 & 0.141 & -.0011494 & .0001656 \\
\hline gdd $\overline{2}$ _genev 1 & $-3.36 e-06$ & .0000257 & -0.13 & 0.896 & -.0000542 & .0000475 \\
\hline cpgd̄_gene 1 & .0000558 & .0003646 & 0.15 & 0.879 & -.000666 & .0007776 \\
\hline Cons & .6250433 & .0214508 & 29.14 & 0.000 & .5825758 & .6675108 \\
\hline
\end{tabular}




\section{Tyloertown, MS}

$\begin{array}{ll}\text { Linear regression } & \text { Number of obs }=154 \\ \mathrm{~F}(32,121) & =1.79 \\ & \text { Prob }>0.0131 \\ \text { R-squared } & =0.3707 \\ \text { Root MSE } & =.04872\end{array}$

\begin{tabular}{|c|c|c|c|c|c|c|}
\hline ndvi_tyler n & Coef. & $\begin{array}{l}\text { Robust HC3 } \\
\text { Std. Err. }\end{array}$ & $t$ & $P>|t|$ & [ $95 \%$ Conf. & Interval] \\
\hline year 1983 & -.0581781 & .0362154 & -1.61 & 0.111 & -.1298759 & .0135198 \\
\hline year_1984 & .02653 & .0220191 & 1.20 & 0.231 & -.0170625 & .0701225 \\
\hline year_1985 & .0268781 & .0251193 & 1.07 & 0.287 & -.0228521 & .0766083 \\
\hline year_1986 & .0113201 & .0224217 & 0.50 & 0.615 & -.0330696 & .0557098 \\
\hline year_1987 & -.0354673 & .0509053 & -0.70 & 0.487 & -.1362477 & .0653132 \\
\hline year_1988 & .0170466 & .0193292 & 0.88 & 0.380 & -.0212206 & .0553139 \\
\hline year_1989 & -.0193976 & .0251847 & -0.77 & 0.443 & -.0692574 & .0304622 \\
\hline year_1990 & .0509098 & .0267369 & 1.90 & 0.059 & -.002023 & .1038426 \\
\hline year_1991 & .0202735 & .021953 & 0.92 & 0.358 & -.0231883 & .0637354 \\
\hline year_1992 & .005863 & .0262942 & 0.22 & 0.824 & -.0461932 & .0579193 \\
\hline year_1993 & .0091782 & .0210911 & 0.44 & 0.664 & -.0325772 & .0509336 \\
\hline year_1994 & -.040543 & .0263546 & -1.54 & 0.127 & -.0927189 & .011633 \\
\hline year_1995 & .0298729 & .0286992 & 1.04 & 0.300 & -.0269447 & .0866905 \\
\hline year_1996 & .007442 & .0299839 & 0.25 & 0.804 & -.051919 & .066803 \\
\hline year_1997 & .0188691 & .02045 & 0.92 & 0.358 & -.0216171 & .0593553 \\
\hline year_1998 & -.0454386 & .0307655 & -1.48 & 0.142 & -.106347 & .0154698 \\
\hline year_1999 & -.032689 & .0375603 & -0.87 & 0.386 & -.1070496 & .0416716 \\
\hline year_2000 & -.0284778 & .0266589 & -1.07 & 0.288 & -.0812561 & .0243005 \\
\hline year_2001 & .0051957 & .0366074 & 0.14 & 0.887 & -.0672784 & .0776697 \\
\hline year_2002 & -.0075366 & .0380494 & -0.20 & 0.843 & -.0828655 & .0677923 \\
\hline year_2003 & .0048576 & .0337951 & 0.14 & 0.886 & -.0620488 & .071764 \\
\hline maȳ1631 & .0001395 & .0152273 & 0.01 & 0.993 & -.0300069 & .0302858 \\
\hline jun115 & -.0273185 & .0202696 & -1.35 & 0.180 & -.0674476 & .0128106 \\
\hline jun 1630 & -.0395705 & .0218987 & -1.81 & 0.073 & -.0829246 & .0037837 \\
\hline jul115 & -.027146 & .0220633 & -1.23 & 0.221 & -.070826 & .0165341 \\
\hline jul1631 & -.029297 & .0223106 & -1.31 & 0.192 & -.0734668 & .0148727 \\
\hline $\operatorname{aug} 115$ & -.0542908 & .0238138 & -2.28 & 0.024 & -.1014364 & -.0071451 \\
\hline plerto s & .0027387 & .0088322 & 0.31 & 0.757 & -.0147469 & .0202243 \\
\hline gdd_tylert s & .0002123 & .0006359 & 0.33 & 0.739 & -.0010467 & .0014714 \\
\hline cp2-tylert s & -.0004498 & .0008281 & -0.54 & 0.588 & -.0020893 & .0011897 \\
\hline gdd $\overline{2}$ tyler s & $-2.43 e-08$ & $3.13 e-06$ & -0.01 & 0.994 & $-6.22 e-06$ & $6.18 e-06$ \\
\hline cpgdd_tyle s & -.0000382 & .0000742 & -0.51 & 0.608 & -.0001851 & .0001088 \\
\hline cons & .7281435 & .0289442 & 25.16 & 0.000 & .6708407 & .7854462 \\
\hline
\end{tabular}




\section{Watervalley, MS}

Linear regression

$\begin{array}{llr}\text { Number of obs } & = & 154 \\ \mathrm{~F}(30,123) & = & 1.53 \\ \text { Prob }>\text { F } & = & 0.0549 \\ \text { R-squared } & =0.2435 \\ \text { Root MSE } & = & .04925\end{array}$

\begin{tabular}{|c|c|c|c|c|c|c|}
\hline ndvi_water y & Coef. & $\begin{array}{l}\text { Robust HC3 } \\
\text { Std. Err. }\end{array}$ & $t$ & $P>|t|$ & [ $95 \%$ Conf. & Interval] \\
\hline year_1983 & -.0407341 & .0176278 & -2.31 & 0.023 & -.0756272 & -.0058409 \\
\hline year_1984 & -.0016127 & .0233541 & -0.07 & 0.945 & -.0478407 & .0446153 \\
\hline year_1985 & -.0026036 & .025339 & -0.10 & 0.918 & -.0527607 & .0475535 \\
\hline year_1986 & -.0318293 & .0223056 & -1.43 & 0.156 & -.0759819 & .0123233 \\
\hline year_1987 & .0097512 & .0235225 & 0.41 & 0.679 & -.0368101 & .0563124 \\
\hline year_1988 & -.007001 & .0317402 & -0.22 & 0.826 & -.0698287 & .0558267 \\
\hline year_1989 & -.0225714 & .0211763 & -1.07 & 0.289 & -.0644886 & .0193459 \\
\hline year_1990 & -.014873 & .0192565 & -0.77 & 0.441 & -.0529901 & .0232441 \\
\hline year_1991 & -.004424 & .0221165 & -0.20 & 0.842 & -.0482022 & .0393542 \\
\hline year_1992 & -.0009264 & .0237348 & -0.04 & 0.969 & -.047908 & .0460552 \\
\hline year_1993 & .0011051 & .0223518 & 0.05 & 0.961 & -.0431389 & .0453492 \\
\hline year_1994 & .0113409 & .0206971 & 0.55 & 0.585 & -.0296278 & .0523095 \\
\hline year_1995 & -.0022643 & .0238139 & -0.10 & 0.924 & -.0494025 & .0448739 \\
\hline year_1996 & .0186871 & .0223299 & 0.84 & 0.404 & -.0255135 & .0628877 \\
\hline year_1997 & .0160506 & .0233264 & 0.69 & 0.493 & -.0301225 & .0622238 \\
\hline year_1998 & .0387544 & .0212002 & 1.83 & 0.070 & -.0032101 & .0807188 \\
\hline year_1999 & -.0545856 & .0298318 & -1.83 & 0.070 & -.1136359 & .0044647 \\
\hline year_2000 & -.0059226 & .0233239 & -0.25 & 0.800 & -.0520909 & .0402456 \\
\hline year_2001 & -.0268454 & .0329803 & -0.81 & 0.417 & -.092128 & .0384371 \\
\hline year_2002 & -.0291613 & .0313492 & -0.93 & 0.354 & -.091215 & .0328925 \\
\hline year_2003 & .0059127 & .0252744 & 0.23 & 0.815 & -.0441164 & .0559418 \\
\hline may1631 & .0197332 & .0166396 & 1.19 & 0.238 & -.0132038 & .0526702 \\
\hline jun115 & .0408046 & .0169469 & 2.41 & 0.018 & .0072593 & .0743499 \\
\hline jun 1630 & .0318378 & .0164458 & 1.94 & 0.055 & -.0007156 & .0643912 \\
\hline jul115 & .0286193 & .0155563 & 1.84 & 0.068 & -.0021735 & .0594121 \\
\hline jul1631 & .0113255 & .0159025 & 0.71 & 0.478 & -.0201525 & .0428034 \\
\hline aug115 & .0110193 & .0160517 & 0.69 & 0.494 & -.020754 & .0427927 \\
\hline aterva s & -.0049316 & .0063898 & -0.77 & 0.442 & -.0175797 & .0077166 \\
\hline gdd_waterv s & -.0370331 & .1677312 & -0.22 & 0.826 & -.3690467 & .2949806 \\
\hline $\begin{array}{l}\text { cp2 waterv } \sim \text { s } \\
\text { gdd2_water s }\end{array}$ & $\begin{array}{r}.0001781 \\
\text { (dropped) }\end{array}$ & .0007825 & 0.23 & 0.820 & -.0013708 & .001727 \\
\hline $\begin{array}{r}\text { gdd_wate } \sim \text { s } \\
\text { cons }\end{array}$ & $\begin{array}{r}\text { (dropped) } \\
.6852792\end{array}$ & .0189837 & 36 & .000 & 7021 & .7228563 \\
\hline -cons & . & & 50.0 & .000 & & $.12<0503$ \\
\hline
\end{tabular}




\section{Booneville, MS}

Linear regression

$\begin{array}{llr}\text { Number of obs } & = & 154 \\ \mathrm{~F}(29,123) & = & . \\ \text { Prob }>\text { F } & = & . \\ \text { R-squared } & = & 0.2516 \\ \text { Root MSE } & = & .06671\end{array}$

\begin{tabular}{|c|c|c|c|c|c|c|}
\hline ndvi_boone e & Coef. & $\begin{array}{l}\text { Robust } \mathrm{HC} 3 \\
\text { Std. Err. }\end{array}$ & $t$ & $P>|t|$ & [95\% Conf. & Interval ] \\
\hline year_1983 & .0176272 & .0288466 & 0.61 & 0.542 & -.0394728 & .0747272 \\
\hline year_1984 & -.0219299 & .0332775 & -0.66 & 0.511 & -.0878007 & .0439409 \\
\hline year_1985 & -.0115745 & .0425277 & -0.27 & 0.786 & -.0957553 & .0726064 \\
\hline year_1986 & -.0431993 & .0396819 & -1.09 & 0.278 & -.1217472 & .0353485 \\
\hline year_1987 & .0267861 & .0240629 & 1.11 & 0.268 & -.0208448 & .0744171 \\
\hline year_1988 & -.0486672 & .0501828 & -0.97 & 0.334 & -.1480009 & .0506665 \\
\hline year_1989 & -.0637051 & .0236603 & -2.69 & 0.008 & -.1105393 & -.0168709 \\
\hline year_1990 & -.0041193 & .0246117 & -0.17 & 0.867 & -.0528368 & .0445981 \\
\hline year_1991 & -.0414676 & .0241906 & -1.71 & 0.089 & -.0893514 & .0064162 \\
\hline year_1992 & .0366734 & .0290355 & 1.26 & 0.209 & -.0208007 & .0941475 \\
\hline year_1993 & .0352452 & .0281077 & 1.25 & 0.212 & -.0203923 & .0908828 \\
\hline year_1994 & -.015201 & .033347 & -0.46 & 0.649 & -.0812093 & .0508072 \\
\hline year_1995 & -.0007134 & .0251597 & -0.03 & 0.977 & -.0505154 & .0490886 \\
\hline year_1996 & -.002436 & .0273371 & -0.09 & 0.929 & -.0565482 & .0516761 \\
\hline year_1997 & -.0299676 & .0509933 & -0.59 & 0.558 & -.1309057 & .0709705 \\
\hline year_1998 & .0534192 & .0338571 & 1.58 & 0.117 & -.0135988 & .1204372 \\
\hline year_1999 & -.0050156 & .0375046 & -0.13 & 0.894 & -.0792536 & .0692223 \\
\hline year_2000 & .0149919 & .0231675 & 0.65 & 0.519 & -.0308668 & .0608507 \\
\hline year_2001 & .0043448 & .0319428 & 0.14 & 0.892 & -.058884 & .0675735 \\
\hline year_2002 & .0464683 & .0347074 & 1.34 & 0.183 & -.0222329 & .1151696 \\
\hline year_2003 & .0353024 & .0287542 & 1.23 & 0.222 & -.0216147 & .0922196 \\
\hline $\operatorname{may} 1631$ & -.0120872 & .0229309 & -0.53 & 0.599 & -.0574774 & .0333031 \\
\hline jun115 & -.0005179 & .0216314 & -0.02 & 0.981 & -.043336 & .0423002 \\
\hline jun 1630 & .0086567 & .0218421 & 0.40 & 0.693 & -.0345783 & .0518917 \\
\hline jul115 & .0153753 & .0215496 & 0.71 & 0.477 & -.0272808 & .0580315 \\
\hline jul1631 & .0153449 & .0188631 & 0.81 & 0.418 & -.0219935 & .0526832 \\
\hline $\operatorname{aug} 115$ & .0368662 & .0189202 & 1.95 & 0.054 & -.0005852 & .0743177 \\
\hline cp_boonevi s & -.0078334 & .0073256 & -1.07 & 0.287 & -.022334 & .0066671 \\
\hline gdd_boonev s & (dropped) & & & & & \\
\hline cp2_boonev s & .0007474 & .0007503 & 1.00 & 0.321 & -.0007378 & .0022327 \\
\hline gdd $\overline{2}$ boone s & -.0117444 & .0038046 & -3.09 & 0.002 & -.0192753 & -.0042134 \\
\hline cpgdd_boon s & (dropped) & & & & & \\
\hline cons & .6702623 & .0236138 & 28.38 & 0.000 & .6235202 & .7170043 \\
\hline
\end{tabular}




\section{Angelica, NY}

Linear regression

$\begin{array}{llr}\text { Number of obs } & = & 147 \\ \text { F }(32,114) & 12.23 \\ \text { Prob }>\text { F } & 0.0000 \\ \text { R-squared } & =0.6782 \\ \text { Root MSE } & = & .06439\end{array}$

\begin{tabular}{|c|c|c|c|c|c|c|}
\hline ndvi_angel $\sim y$ & Coef. & $\begin{array}{c}\text { Robust } \mathrm{HC} 3 \\
\text { Std. Err. }\end{array}$ & $t$ & $P>|t|$ & [95\% Conf. & Interval] \\
\hline year_1983 & -.0283738 & .0481552 & -0.59 & 0.557 & -.1237689 & .0670212 \\
\hline year-1984 & .0081673 & .0291335 & 0.28 & 0.780 & -.0495459 & .0658805 \\
\hline year_1985 & .0641509 & .0319778 & 2.01 & 0.047 & .0008031 & .1274987 \\
\hline year 1986 & -.0114171 & .0532736 & -0.21 & 0.831 & -.1169518 & .0941176 \\
\hline year-1987 & .0901469 & .0291259 & 3.10 & 0.002 & .0324486 & .1478451 \\
\hline year 1988 & .0367367 & .0335771 & 1.09 & 0.276 & -.0297792 & .1032526 \\
\hline year 1989 & -.0041931 & .0478049 & -0.09 & 0.930 & -.0988942 & .0905081 \\
\hline year-1990 & -.0021799 & .0326676 & -0.07 & 0.947 & -.0668942 & .0625345 \\
\hline year-1991 & .061097 & .0306541 & 1.99 & 0.049 & .0003715 & .1218226 \\
\hline year 1992 & .0138988 & .0374164 & 0.37 & 0.711 & -.0602229 & .0880204 \\
\hline year-1993 & .0402421 & .0410566 & 0.98 & 0.329 & -.0410908 & .121575 \\
\hline year-1994 & .029428 & .0336716 & 0.87 & 0.384 & -.0372752 & .0961312 \\
\hline year_1995 & .0346229 & .0412579 & 0.84 & 0.403 & -.0471087 & .1163545 \\
\hline year_1996 & .011258 & .0408926 & 0.28 & 0.784 & -.0697499 & .0922659 \\
\hline year_1997 & .0805086 & .0528843 & 1.52 & 0.131 & -.0242548 & .185272 \\
\hline year-1998 & .0304986 & .0358385 & 0.85 & 0.397 & -.0404972 & .1014944 \\
\hline year-1999 & -.1151762 & .0687459 & -1.68 & 0.097 & -.2513613 & .0210089 \\
\hline year_2000 & .0122428 & .0420215 & 0.29 & 0.771 & -.0710016 & .0954871 \\
\hline year 2001 & .0405299 & .0333724 & 1.21 & 0.227 & -.0255806 & .1066404 \\
\hline year 2002 & .022705 & .0309884 & 0.73 & 0.465 & -.0386828 & .0840928 \\
\hline year 2003 & -.0402226 & .0392238 & -1.03 & 0.307 & -.1179246 & .0374795 \\
\hline $\operatorname{may} 1631$ & .1194912 & .0191495 & 6.24 & 0.000 & .0815563 & .1574261 \\
\hline jun 115 & .1781642 & .0257993 & 6.91 & 0.000 & .1270559 & .2292724 \\
\hline jun 1630 & .1995968 & .0207513 & 9.62 & 0.000 & .1584886 & .240705 \\
\hline jul115 & .2048994 & .0203338 & 10.08 & 0.000 & .1646182 & .2451805 \\
\hline jul1631 & .2053064 & .0254659 & 8.06 & 0.000 & .1548586 & .2557542 \\
\hline $\operatorname{aug} 115$ & .1875534 & .0213777 & 8.77 & 0.000 & .1452043 & .2299026 \\
\hline gelic $\sim y$ & -.0214638 & .013715 & -1.56 & 0.120 & -.0486331 & .0057054 \\
\hline gdd_angeli $\sim y$ & -.0059557 & .0149817 & -0.40 & 0.692 & -.0356344 & .023723 \\
\hline cp2 angeli y & .0034728 & .0021568 & 1.61 & 0.110 & -.0007999 & .0077455 \\
\hline gdd $\overline{2}$ angel $\sim \mathrm{y}$ & .0002725 & .0008616 & 0.32 & 0.752 & -.0014344 & .0019794 \\
\hline pgdd_ange $\sim \mathrm{y}$ & .0051141 & .0042287 & 1.21 & 0.229 & -.003263 & .0134911 \\
\hline cons & .6012895 & .0335662 & 17.91 & 0.000 & .5347951 & .667784 \\
\hline
\end{tabular}




\title{
Riverhead, NY
}

\author{
Linear regression
}

$\begin{array}{llr}\text { Number of obs } & & 153 \\ \text { F }(32, \text { 120) } & 6.66 \\ \text { Prob F } & =0000 \\ \text { R-squared } & =0.4179 \\ \text { Root MSE } & = & .05856\end{array}$

\begin{tabular}{|c|c|c|c|c|c|c|}
\hline ndvi_river y & Coef. & $\begin{array}{l}\text { Robust } \mathrm{HC} 3 \\
\text { Std. Err. }\end{array}$ & $t$ & $P>|t|$ & [ $95 \%$ Conf. & Interval] \\
\hline year 1983 & 0416971 & 0471456 & 088 & 0378 & 051648 & 1350421 \\
\hline year 1984 & -.020925 & .0304148 & -0.69 & 0.493 & $\begin{array}{r}-.051648 \\
-.0811442\end{array}$ & $\begin{array}{l}.1350421 \\
.0392942\end{array}$ \\
\hline year 1985 & -.054676 & .0297978 & -1.83 & 0.069 & -.1136736 & .0043215 \\
\hline year_1986 & -.0553459 & .0294918 & -1.88 & 0.063 & -.1137375 & .0030458 \\
\hline year_1987 & -.0619271 & .0277143 & -2.23 & 0.027 & -.1167996 & -.0070547 \\
\hline year_1988 & -.0563372 & .0286758 & -1.96 & 0.052 & -.1131132 & .0004388 \\
\hline year_1989 & -.0433322 & .0316512 & -1.37 & 0.174 & -.1059994 & .0193351 \\
\hline year-1990 & -.0315593 & .0897602 & -0.35 & 0.726 & -.2092783 & .1461596 \\
\hline year_1991 & -.0504409 & .0289853 & -1.74 & 0.084 & -.1078298 & .006948 \\
\hline year_1992 & -.00971 & .0466531 & -0.21 & 0.835 & -.10208 & .0826599 \\
\hline year 1993 & -.0481086 & .0283864 & -1.69 & 0.093 & -.1043117 & .0080945 \\
\hline year_1994 & -.0662178 & .0291813 & -2.27 & 0.025 & -.1239948 & -.0084408 \\
\hline year_1995 & -.0560783 & .029241 & -1.92 & 0.058 & -.1139736 & .0018169 \\
\hline year_1996 & -.039141 & .0286568 & -1.37 & 0.175 & -.0958795 & .0175976 \\
\hline year_1997 & -.0416685 & .0279285 & -1.49 & 0.138 & -.096965 & .0136279 \\
\hline year_1998 & -.0339818 & .0286608 & -1.19 & 0.238 & -.0907281 & .0227646 \\
\hline year_1999 & -.0406091 & .0282846 & -1.44 & 0.154 & -.0966106 & .0153923 \\
\hline year_2000 & -.0608872 & .0297703 & -2.05 & 0.043 & -.1198303 & -.0019441 \\
\hline year_2001 & -.0239945 & .0314857 & -0.76 & 0.448 & -.0863341 & .0383451 \\
\hline year_2002 & -.0071209 & .0346145 & -0.21 & 0.837 & -.0756551 & .0614134 \\
\hline year_2003 & -.0434661 & .0332109 & -1.31 & 0.193 & -.1092213 & .0222892 \\
\hline $\operatorname{may} 1631$ & .0236187 & .0172444 & 1.37 & 0.173 & -.010524 & .0577614 \\
\hline jun 115 & .0347223 & .0234769 & 1.48 & 0.142 & -.0117604 & .081205 \\
\hline jun 1630 & .0028936 & .0211363 & 0.14 & 0.891 & -.0389548 & .0447419 \\
\hline jul115 & .0633983 & .0246468 & 2.57 & 0.011 & .0145994 & .1121973 \\
\hline jul1631 & .0995204 & .0232477 & 4.28 & 0.000 & .0534917 & .1455492 \\
\hline $\operatorname{aug} 115$ & .0754067 & .0172465 & 4.37 & 0.000 & .0412599 & .1095535 \\
\hline cp_riverhe $\mathrm{y}$ & .0005499 & .0103395 & 0.05 & 0.958 & -.0199216 & .0210214 \\
\hline dd_riverh y & .000076 & .0008351 & 0.09 & 0.928 & -.0015774 & .0017294 \\
\hline riverh $\sim y$ & -.0008595 & .0008545 & -1.01 & 0.316 & -.0025513 & .0008323 \\
\hline dd $\overline{2}$ _river $\sim y$ & $-2.92 e-07$ & $7.03 e-06$ & -0.04 & 0.967 & -.0000142 & .0000136 \\
\hline gdd__rive $y$ & $-3.38 e-06$ & .0001283 & -0.03 & 0.979 & -.0002574 & .0002506 \\
\hline cons & .2337028 & .0289396 & 8.08 & 0.000 & .1764044 & .2910012 \\
\hline
\end{tabular}




\section{Dixon Springs, IL}

Linear regression

$\begin{array}{llr}\text { Number of obs } & = & 154 \\ \mathrm{~F}(32,121) & = & 2.73 \\ \text { Prob }>\mathrm{F} & = & 0.0000 \\ \text { R-squared } & = & 0.3850 \\ \text { Root MSE } & = & .0511\end{array}$

\begin{tabular}{|c|c|c|c|c|c|c|}
\hline ndvi_dixon 1 & Coef. & $\begin{array}{l}\text { Robust } \mathrm{HC} 3 \\
\text { Std. Err. }\end{array}$ & $t$ & $P>|t|$ & [95\% Con & Inte \\
\hline year_1983 & -.0271273 & .0260684 & -1.04 & 0.300 & -.0787366 & .0244821 \\
\hline year_1984 & -.0050628 & .0250265 & -0.20 & 0.840 & -.0546093 & .0444837 \\
\hline year_1985 & .0189454 & .0280572 & 0.68 & 0.501 & -.0366013 & .0744921 \\
\hline year_1986 & -.0426464 & .0256717 & -1.66 & 0.099 & -.0934703 & .0081775 \\
\hline year_1987 & .0021229 & .0165704 & 0.13 & 0.898 & -.0306826 & .0349283 \\
\hline year_1988 & -.0370846 & .0326293 & -1.14 & 0.258 & -.1016828 & .0275137 \\
\hline year_1989 & -.0235597 & .0193192 & -1.22 & 0.225 & -.0618071 & .0146877 \\
\hline year_1990 & -.0122354 & .0328022 & -0.37 & 0.710 & -.077176 & .0527051 \\
\hline year_1991 & -.0506332 & .021018 & -2.41 & 0.018 & -.0922439 & -.0090225 \\
\hline year_1992 & -.0719529 & .0290269 & -2.48 & 0.015 & -.1294192 & -.0144865 \\
\hline year_1993 & .001795 & .0218586 & 0.08 & 0.935 & -.0414799 & .0450699 \\
\hline year_1994 & -.0084006 & .0305541 & -0.27 & 0.784 & -.0688904 & .0520893 \\
\hline year_1995 & -.0238949 & .0192098 & -1.24 & 0.216 & -.0619257 & .014136 \\
\hline year_1996 & -.047496 & .0258983 & -1.83 & 0.069 & -.0987685 & .0037765 \\
\hline year_1997 & -.0363165 & .0189991 & -1.91 & 0.058 & -.0739301 & .0012972 \\
\hline year_1998 & -.0173899 & .017725 & -0.98 & 0.329 & -.0524813 & .0177015 \\
\hline year_1999 & -.0841162 & .0381953 & -2.20 & 0.030 & -.1597339 & -.0084984 \\
\hline year_2000 & -.0456207 & .0184752 & -2.47 & 0.015 & -.0821973 & -.009044 \\
\hline year_2001 & -.0514956 & .0270189 & -1.91 & 0.059 & -.1049867 & .0019954 \\
\hline year_2002 & -.0700223 & .0399773 & -1.75 & 0.082 & -.1491679 & .0091234 \\
\hline year_2003 & -.0509445 & .0207575 & -2.45 & 0.016 & -.0920396 & -.0098495 \\
\hline maȳ1631 & .0203427 & .0210306 & 0.97 & 0.335 & -.021293 & .0619783 \\
\hline jun 115 & .0490718 & .0188481 & 2.60 & 0.010 & .0117571 & .0863866 \\
\hline jun 1630 & .0617864 & .0218323 & 2.83 & 0.005 & .0185636 & .1050092 \\
\hline jul115 & .0343052 & .025966 & 1.32 & 0.189 & -.0171015 & .0857118 \\
\hline jul1631 & .0160639 & .028707 & 0.56 & 0.577 & -.0407692 & .072897 \\
\hline $\operatorname{aug} 115$ & .0166231 & .0236139 & 0.70 & 0.483 & -.0301269 & .0633731 \\
\hline ixonsp 1 & -.0247274 & .0149805 & -1.65 & 0.101 & -.0543853 & .0049306 \\
\hline gdd_dixons $\sim 1$ & .0001348 & .0006875 & 0.20 & 0.845 & -.0012264 & .0014959 \\
\hline cp2_dixons 1 & .0023076 & .0015415 & 1.50 & 0.137 & -.0007442 & .0053594 \\
\hline gddי2_dixon 1 & $-1.36 e-06$ & $3.33 e-06$ & -0.41 & 0.683 & $-7.96 e-06$ & $5.23 e-06$ \\
\hline $\bar{d} \_d i x o \sim 1$ & .0000739 & .0001263 & 0.59 & 0.559 & -.0001761 & .000324 \\
\hline _cons & .7767418 & .0306565 & 25.34 & 0.000 & .7160492 & .8374343 \\
\hline
\end{tabular}


Cooperstown, NY

Linear regression

$\begin{array}{llr}\text { Number of obs } & = & 152 \\ \mathrm{~F}(32, \text { 119) } & = & 9.14 \\ \text { Prob }>\text { F } & = & 0.0000 \\ \text { R-squared } & = & 0.7375 \\ \text { Root MSE } & = & .05264\end{array}$

\begin{tabular}{|c|c|c|c|c|c|c|}
\hline ndvi_coope $\sim y$ & Coef. & $\begin{array}{l}\text { Robust } \mathrm{HC} 3 \\
\text { Std. Err. }\end{array}$ & $t$ & $P>|t|$ & [95\% Conf. & Inte \\
\hline year_1983 & -.0261502 & .0628468 & -0.42 & 0.678 & -.150593 & .0982927 \\
\hline year_1984 & -.0273081 & .0402466 & -0.68 & 0.499 & -.1070004 & .0523843 \\
\hline year_1985 & -.0007546 & .0412589 & -0.02 & 0.985 & -.0824513 & .0809421 \\
\hline year_1986 & -.0133495 & .0468885 & -0.28 & 0.776 & -.1061933 & .0794944 \\
\hline year_1987 & .0526468 & .0375965 & 1.40 & 0.164 & -.0217981 & .1270917 \\
\hline year_1988 & -.0730128 & .0467429 & -1.56 & 0.121 & -.1655685 & .0195428 \\
\hline year_1989 & -.0068823 & .0413784 & -0.17 & 0.868 & -.0888157 & .0750512 \\
\hline year_1990 & .0275128 & .0390083 & 0.71 & 0.482 & -.0497277 & .1047532 \\
\hline year_1991 & .0358906 & .0408731 & 0.88 & 0.382 & -.0450423 & .1168235 \\
\hline year_1992 & .0155663 & .0377681 & 0.41 & 0.681 & -.0592184 & .0903509 \\
\hline year_1993 & -.002513 & .0430087 & -0.06 & 0.954 & -.0876744 & .0826484 \\
\hline year_1994 & -.0455109 & .0428122 & -1.06 & 0.290 & -.1302833 & .0392615 \\
\hline year_1995 & .0109622 & .0414536 & 0.26 & 0.792 & -.07112 & .0930443 \\
\hline year_1996 & .0016894 & .0410118 & 0.04 & 0.967 & -.079518 & .0828968 \\
\hline year_1997 & .0091859 & .0423236 & 0.22 & 0.829 & -.074619 & .0929908 \\
\hline year_1998 & -.0150007 & .0493353 & -0.30 & 0.762 & -.1126896 & .0826882 \\
\hline year_1999 & -.0612155 & .0465244 & -1.32 & 0.191 & -.1533385 & .0309076 \\
\hline year_2000 & -.0229831 & .0396455 & -0.58 & 0.563 & -.1014851 & .0555189 \\
\hline year_2001 & -.0003435 & .0388129 & -0.01 & 0.993 & -.0771968 & .0765099 \\
\hline year_2002 & -.0241873 & .0400325 & -0.60 & 0.547 & -.1034556 & .055081 \\
\hline year_2003 & -.0259923 & .0381602 & -0.68 & 0.497 & -.1015534 & .0495688 \\
\hline maȳ1631 & .1086838 & .0218349 & 4.98 & 0.000 & .0654485 & .1519191 \\
\hline jun 115 & .1820393 & .0187081 & 9.73 & 0.000 & .1449954 & .2190832 \\
\hline jun 1630 & .2126047 & .0195842 & 10.86 & 0.000 & .1738261 & .2513834 \\
\hline jul115 & .2114519 & .019498 & 10.84 & 0.000 & .172844 & .2500599 \\
\hline jul1631 & .2029356 & .0207677 & 9.77 & 0.000 & .1618135 & .2440578 \\
\hline $\operatorname{aug} 115$ & .19877 & .0221483 & 8.97 & 0.000 & .1549142 & .2426258 \\
\hline opers $\sim y$ & -.0156301 & .0165597 & -0.94 & 0.347 & -.0484199 & .0171597 \\
\hline gdd__cooper y & -.0036227 & .0070859 & -0.51 & 0.610 & -.0176535 & .010408 \\
\hline cooper y & .0026359 & .0031953 & 0.82 & 0.411 & -.0036912 & .008963 \\
\hline gdd'2_coope $\sim$ y & .00013 & .0003102 & 0.42 & 0.676 & -.0004843 & .0007442 \\
\hline gdd__coop $\sim y$ & .0008839 & .0020455 & 0.43 & 0.666 & -.0031664 & .0049341 \\
\hline _cons & .6405323 & .0421319 & 15.20 & 0.000 & .5571069 & .7239577 \\
\hline
\end{tabular}


Fredonia, NY

Linear regression

$\begin{array}{llr}\text { Number of obs } & = & 150 \\ \text { F }(32, \text { 117) } & 5.31 \\ \text { Prob }>\text { F } & 0.0000 \\ \text { R-squared } & =0.4680 \\ \text { Root MSE } & = & .13475\end{array}$

\begin{tabular}{|c|c|c|c|c|c|c|}
\hline ndvi_fredo y & Coef. & $\begin{array}{c}\text { Robust HC3 } \\
\text { Std. Err. }\end{array}$ & $t$ & $P>|t|$ & [95\% Conf. & Inte \\
\hline year_1983 & .2327651 & .0655209 & 3.55 & 0.001 & .1030043 & .3625258 \\
\hline year_1984 & -.0675862 & .0589121 & -1.15 & 0.254 & -.1842584 & .0490861 \\
\hline year_1985 & .0421883 & .086927 & 0.49 & 0.628 & -.1299661 & .2143426 \\
\hline year_1986 & .0620425 & .0935701 & 0.66 & 0.509 & -.1232682 & .2473531 \\
\hline year_1987 & .0972161 & .1161419 & 0.84 & 0.404 & -.1327969 & .3272292 \\
\hline year_1988 & .0296327 & .1196946 & 0.25 & 0.805 & -.2074163 & .2666816 \\
\hline year_1989 & -.0965262 & .0529252 & -1.82 & 0.071 & -.2013418 & .0082893 \\
\hline year_1990 & .2298279 & .096938 & 2.37 & 0.019 & .0378472 & .4218086 \\
\hline year_1991 & .1488724 & .076411 & 1.95 & 0.054 & -.0024556 & .3002004 \\
\hline year_1992 & -.0557951 & .0597568 & -0.93 & 0.352 & -.1741404 & .0625502 \\
\hline year_1993 & -.0659511 & .0658079 & -1.00 & 0.318 & -.1962801 & .0643779 \\
\hline year_1994 & -.0581795 & .0644554 & -0.90 & 0.369 & -.18583 & .0694709 \\
\hline year_1995 & .2286902 & .0725724 & 3.15 & 0.002 & .0849644 & .372416 \\
\hline year_1996 & .0935393 & .087786 & 1.07 & 0.289 & -.0803162 & .2673948 \\
\hline year_1997 & .2181918 & .0705528 & 3.09 & 0.002 & .0784657 & .3579179 \\
\hline year_1998 & .0696453 & .0861659 & 0.81 & 0.421 & -.1010018 & .2402923 \\
\hline year_1999 & .1012545 & .0980691 & 1.03 & 0.304 & -.0929662 & .2954752 \\
\hline year_2000 & -.0427883 & .0575581 & -0.74 & 0.459 & -.1567791 & .0712024 \\
\hline year_2001 & .0926657 & .0488017 & 1.90 & 0.060 & -.0039836 & .1893149 \\
\hline year_2002 & .0925211 & .0558124 & 1.66 & 0.100 & -.0180125 & .2030547 \\
\hline year_2003 & .0588423 & .0486616 & 1.21 & 0.229 & -.0375295 & .1552142 \\
\hline $\operatorname{may} 1631$ & .0635006 & .0407635 & 1.56 & 0.122 & -.0172294 & .1442306 \\
\hline jun 115 & .1138036 & .0452602 & 2.51 & 0.013 & .0241683 & .203439 \\
\hline jun 1630 & .1170005 & .0499729 & 2.34 & 0.021 & .0180318 & .2159692 \\
\hline jul115 & .0848134 & .0464877 & 1.82 & 0.071 & -.0072532 & .1768799 \\
\hline jul1631 & .1165217 & .0539467 & 2.16 & 0.033 & .0096831 & .2233602 \\
\hline $\operatorname{aug} 115$ & .1514606 & .0415745 & 3.64 & 0.000 & .0691243 & .2337968 \\
\hline fredoni y & .0036334 & .036792 & 0.10 & 0.922 & -.0692313 & .0764981 \\
\hline gdd_fredon $\sim y$ & -.0025888 & .0042545 & -0.61 & 0.544 & -.0110145 & .0058369 \\
\hline cp2_fredon $\sim y$ & -.0037338 & .0076721 & -0.49 & 0.627 & -.0189281 & .0114604 \\
\hline gdd $\overline{2}$ fredo $y$ & .0000173 & .0000853 & 0.20 & 0.840 & -.0001516 & .0001862 \\
\hline ogdd̄_fred $\sim \bar{y}$ & .0007017 & .0012981 & 0.54 & 0.590 & -.0018691 & .0032725 \\
\hline _cons & .2131806 & .0639232 & 3.33 & 0.001 & .0865841 & .3397771 \\
\hline
\end{tabular}




\section{DuQuoin, IL}

Linear regression

$\begin{array}{llr}\text { Number of obs } & & 152 \\ \text { F }(32,119) & 3.06 \\ \text { Prob }>\text { F } & =.0000 \\ \text { R-squared } & =0.4759 \\ \text { Root MSE } & = & .06219\end{array}$

\begin{tabular}{|c|c|c|c|c|c|c|}
\hline ndvi_duquo 1 & Coef. & $\begin{array}{c}\text { Robust HC3 } \\
\text { Std. Err. }\end{array}$ & $t$ & $P>|t|$ & [ $95 \%$ Conf. & Int \\
\hline year_1983 & -.0715632 & .0301413 & -2.37 & 0.019 & -.131246 & -.0118805 \\
\hline year_1984 & -.0123733 & .0351284 & -0.35 & 0.725 & -.081931 & .0571844 \\
\hline year_1985 & -.0467007 & .0364259 & -1.28 & 0.202 & -.1188276 & .0254261 \\
\hline year_1986 & -.0825284 & .0505133 & -1.63 & 0.105 & -.1825498 & .0174931 \\
\hline year_1987 & -.0151675 & .030946 & -0.49 & 0.625 & -.0764436 & .0461086 \\
\hline year_1988 & -.0578558 & .0436535 & -1.33 & 0.188 & -.1442942 & .0285826 \\
\hline year_1989 & -.0518532 & .0302361 & -1.71 & 0.089 & -.1117237 & .0080174 \\
\hline year_1990 & -.0293687 & .0292072 & -1.01 & 0.317 & -.0872019 & .0284645 \\
\hline year_1991 & .0070318 & .0310087 & 0.23 & 0.821 & -.0543684 & .0684321 \\
\hline year_1992 & -.0497954 & .0329006 & -1.51 & 0.133 & -.1149418 & .015351 \\
\hline year_1993 & .0096508 & .0277434 & 0.35 & 0.729 & -.045284 & .0645855 \\
\hline year_1994 & .0456559 & .0360946 & 1.26 & 0.208 & -.025815 & .1171268 \\
\hline year_1995 & -.0620663 & .029541 & -2.10 & 0.038 & -.1205604 & -.0035721 \\
\hline year_1996 & -.0457834 & .0367818 & -1.24 & 0.216 & -.1186151 & .0270482 \\
\hline year_1997 & -.0208775 & .0280698 & -0.74 & 0.458 & -.0764585 & .0347036 \\
\hline year_1998 & -.0494999 & .035866 & -1.38 & 0.170 & -.1205182 & .0215184 \\
\hline year_1999 & -.0730326 & .0554995 & -1.32 & 0.191 & -.1829273 & .036862 \\
\hline year_2000 & -.0163926 & .0323894 & -0.51 & 0.614 & -.0805268 & .0477415 \\
\hline year_2001 & .0369799 & .052815 & 0.70 & 0.485 & -.067599 & .1415588 \\
\hline year_2002 & -.1073475 & .0363553 & -2.95 & 0.004 & -.1793347 & -.0353603 \\
\hline year_2003 & -.0833309 & .0304973 & -2.73 & 0.007 & -.1437185 & -.0229432 \\
\hline $\operatorname{may} 1631$ & .0021801 & .0250778 & 0.09 & 0.931 & -.0474764 & .0518367 \\
\hline jun115 & .0267184 & .0204057 & 1.31 & 0.193 & -.0136868 & .0671237 \\
\hline jun 1630 & .0283487 & .024868 & 1.14 & 0.257 & -.0208923 & .0775898 \\
\hline jul115 & .0450466 & .0272282 & 1.65 & 0.101 & -.0088679 & .0989612 \\
\hline jul1631 & .066614 & .0223626 & 2.98 & 0.004 & .0223337 & .1108943 \\
\hline $\operatorname{aug} 115$ & .0966548 & .0239181 & 4.04 & 0.000 & .0492945 & .1440151 \\
\hline dquoinil & -.003905 & .0106682 & -0.37 & 0.715 & -.0250292 & .0172191 \\
\hline gdd_duquoi l & .0030754 & .0043228 & 0.71 & 0.478 & -.0054842 & .0116349 \\
\hline cp2_duquoi 1 & .0006234 & .0010111 & 0.62 & 0.539 & -.0013787 & .0026256 \\
\hline gdd 2 _duquo 1 & -.0001246 & .0001258 & -0.99 & 0.324 & -.0003737 & .0001245 \\
\hline cpgdd̄_duqu 1 & .0003616 & .0011602 & 0.31 & 0.756 & -.0019357 & .0026588 \\
\hline cons & .6569405 & .0347298 & 18.92 & 0.000 & .588172 & .725709 \\
\hline
\end{tabular}




\section{Minonk, IL}

Linear regression

$\begin{array}{llr}\text { Number of obs } & & 154 \\ \text { F }(32, \text { 121) } & 42.21 \\ \text { Prob F } & =0.0000 \\ \text { R-squared } & =0.8804 \\ \text { Root MSE } & =.08085\end{array}$

\begin{tabular}{|c|c|c|c|c|c|c|}
\hline ndvi_minon 1 & Coef. & $\begin{array}{l}\text { Robust HC3 } \\
\text { Std. Err. }\end{array}$ & $t$ & $P>|t|$ & [95\% Conf. & Interval] \\
\hline & & & & & & \\
\hline year_1983 & .0481888 & .0447709 & 1.08 & 0.284 & -.0404471 & .1368247 \\
\hline year_1984 & .0922199 & .0429529 & 2.15 & 0.034 & .0071834 & .1772564 \\
\hline year_1985 & .0119913 & .0521421 & 0.23 & 0.819 & -.0912377 & .1152204 \\
\hline year_1986 & .1167071 & .0473162 & 2.47 & 0.015 & .0230322 & .2103819 \\
\hline year_1987 & .1414166 & .064887 & 2.18 & 0.031 & .0129557 & .2698775 \\
\hline year_1988 & .03183 & .0550482 & 0.58 & 0.564 & -.0771523 & .1408124 \\
\hline year_1989 & .0146755 & .0510324 & 0.29 & 0.774 & -.0863567 & .1157077 \\
\hline year_1990 & .0107141 & .0462373 & 0.23 & 0.817 & -.0808249 & .1022531 \\
\hline year_1991 & .0900767 & .0526151 & 1.71 & 0.089 & -.0140888 & .1942423 \\
\hline year_1992 & .0439921 & .0585647 & 0.75 & 0.454 & -.0719522 & .1599365 \\
\hline year_1993 & .1098077 & .0385648 & 2.85 & 0.005 & .0334584 & .1861569 \\
\hline year_1994 & .114623 & .038932 & 2.94 & 0.004 & .0375468 & .1916993 \\
\hline year_1995 & -.0468025 & .0737909 & -0.63 & 0.527 & -.192891 & .0992861 \\
\hline year_1996 & .0212617 & .0515383 & 0.41 & 0.681 & -.0807719 & .1232953 \\
\hline year_1997 & .0730018 & .039306 & 1.86 & 0.066 & -.0048148 & .1508184 \\
\hline year_1998 & .0320344 & .0442703 & 0.72 & 0.471 & -.0556103 & .1196791 \\
\hline year_1999 & .0092114 & .0470031 & 0.20 & 0.845 & -.0838435 & .1022664 \\
\hline year_2000 & .0490382 & .0526729 & 0.93 & 0.354 & -.0552416 & .1533181 \\
\hline year_2001 & .0514822 & .0491554 & 1.05 & 0.297 & -.0458339 & .1487983 \\
\hline year_2002 & -.014293 & .0478542 & -0.30 & 0.766 & -.109033 & .0804471 \\
\hline year_2003 & -.0073834 & .0466717 & -0.16 & 0.875 & -.0997823 & .0850154 \\
\hline $\operatorname{may} 1631$ & .0461896 & .0216479 & 2.13 & 0.035 & .0033319 & .0890474 \\
\hline jun115 & .163816 & .0219158 & 7.47 & 0.000 & .120428 & .2072041 \\
\hline jun 1630 & .2993712 & .0253612 & 11.80 & 0.000 & .2491621 & .3495804 \\
\hline jul115 & .4384863 & .0227429 & 19.28 & 0.000 & .3934607 & .4835118 \\
\hline jul1631 & .4785873 & .0305599 & 15.66 & 0.000 & .418086 & .5390886 \\
\hline $\operatorname{aug} 115$ & .4710056 & .0268054 & 17.57 & 0.000 & .4179372 & .5240741 \\
\hline cp_minonkil & -.0035669 & .0193004 & -0.18 & 0.854 & -.041777 & .0346433 \\
\hline gdd-minonkil & -.0550987 & .0975211 & -0.56 & 0.573 & -.2481675 & .1379701 \\
\hline cp2_minonkil & .0004504 & .003925 & 0.11 & 0.909 & -.0073202 & .008221 \\
\hline gdd $\overline{2}$ _minon 1 & .0098144 & .0350977 & 0.28 & 0.780 & -.0596707 & .0792995 \\
\hline cpgdd__mino 1 & .0066651 & .054316 & 0.12 & 0.903 & -.1008678 & .114198 \\
\hline _cons & .2900124 & .0439497 & 6.60 & 0.000 & .2030023 & .3770224 \\
\hline
\end{tabular}




\section{Ardmore, SD}

Linear regression

$\begin{array}{llr}\text { Number of obs } & = & 154 \\ \mathrm{~F}(32, \text { 121) } & = & 7.23 \\ \text { Prob }>\text { F } & =.0000 \\ \text { R-squared } & =0.6931 \\ \text { Root MSE } & = & .07427\end{array}$

\begin{tabular}{|c|c|c|c|c|c|c|}
\hline ndvi_ardmo d & Coef. & $\begin{array}{c}\text { Robust HC3 } \\
\text { Std. Err. }\end{array}$ & $t$ & $P>|t|$ & {$[95 \%$ Conf } & Int \\
\hline year_1983 & .0474251 & .0507304 & 0.93 & 0.352 & -.0530091 & .1478592 \\
\hline year_1984 & .0555745 & .0484905 & 1.15 & 0.254 & -.0404253 & .1515743 \\
\hline year_1985 & -.1493896 & .0450467 & -3.32 & 0.001 & -.2385714 & -.0602078 \\
\hline year_1986 & .0262037 & .037659 & 0.70 & 0.488 & -.0483523 & .1007597 \\
\hline year_1987 & -.0158939 & .0489111 & -0.32 & 0.746 & -.1127263 & .0809386 \\
\hline year_1988 & -.0775526 & .040946 & -1.89 & 0.061 & -.1586159 & .0035108 \\
\hline year_1989 & -.1992094 & .0551606 & -3.61 & 0.000 & -.3084143 & -.0900045 \\
\hline year_1990 & -.084743 & .0367785 & -2.30 & 0.023 & -.1575558 & -.0119302 \\
\hline year_1991 & .0304762 & .041676 & 0.73 & 0.466 & -.0520324 & .1129849 \\
\hline year_1992 & -.0482084 & .0495442 & -0.97 & 0.332 & -.1462941 & .0498773 \\
\hline year_1993 & .0864502 & .0418444 & 2.07 & 0.041 & .0036082 & .1692922 \\
\hline year_1994 & -.0208586 & .0357225 & -0.58 & 0.560 & -.0915808 & .0498636 \\
\hline year_1995 & .029145 & .0432149 & 0.67 & 0.501 & -.0564102 & .1147002 \\
\hline year_1996 & -.0390643 & .0368469 & -1.06 & 0.291 & -.1120126 & .0338839 \\
\hline year_1997 & -.0400871 & .0392234 & -1.02 & 0.309 & -.1177402 & .0375661 \\
\hline year_1998 & -.0504032 & .0476944 & -1.06 & 0.293 & -.1448269 & .0440204 \\
\hline year_1999 & .0389671 & .0474788 & 0.82 & 0.413 & -.0550298 & .132964 \\
\hline year_2000 & -.0186798 & .0486165 & -0.38 & 0.701 & -.114929 & .0775693 \\
\hline year_2001 & .0603024 & .0414972 & 1.45 & 0.149 & -.0218522 & .142457 \\
\hline year_2002 & -.0852921 & .0473224 & -1.80 & 0.074 & -.1789794 & .0083951 \\
\hline year_2003 & .0394685 & .0539818 & 0.73 & 0.466 & -.0674028 & .1463398 \\
\hline $\operatorname{may} 1631$ & .0950105 & .0253219 & 3.75 & 0.000 & .044879 & .145142 \\
\hline jun115 & .1584467 & .0247595 & 6.40 & 0.000 & .1094288 & .2074646 \\
\hline jun 1630 & .1565548 & .0247249 & 6.33 & 0.000 & .1076054 & .2055042 \\
\hline jul115 & .1172997 & .0287907 & 4.07 & 0.000 & .0603009 & .1742984 \\
\hline jull631 & .0920791 & .0291283 & 3.16 & 0.002 & .034412 & .1497463 \\
\hline $\operatorname{aug} 115$ & .0223606 & .0325038 & 0.69 & 0.493 & -.0419892 & .0867104 \\
\hline rdmoresd & -.0243216 & .0149466 & -1.63 & 0.106 & -.0539123 & .005269 \\
\hline gdd_ardmor $\sim d$ & -.0037858 & .0025508 & -1.48 & 0.140 & -.0088359 & .0012643 \\
\hline cp2_ardmor d & .0033668 & .0029678 & 1.13 & 0.259 & -.0025087 & .0092423 \\
\hline gdd $\overline{2}$ _ardmo d & .0000266 & .0000488 & 0.55 & 0.586 & -.00007 & .0001233 \\
\hline ogdd__ardm d & .0000814 & .0007241 & 0.11 & 0.911 & -.0013521 & .0015149 \\
\hline _cons & .4557017 & .0375284 & 12.14 & 0.000 & .3814043 & .529999 \\
\hline
\end{tabular}


Aledo, IL

Linear regression

Number of obs =

147

114) $=19.33$

Prob $>\mathrm{F} \quad=0.0000$

R-squared $=0.8066$

Root MSE $=.06254$

\begin{tabular}{|c|c|c|c|c|c|c|}
\hline ndvi_aledoil & Coef. & $\begin{array}{c}\text { Robust } \mathrm{HC} 3 \\
\text { Std. Err. }\end{array}$ & $t$ & $P>|t|$ & [95\% Conf. & Interval] \\
\hline year_1983 & .040315 & .0329944 & 1.22 & 0.224 & -.0250466 & .1056766 \\
\hline year 1984 & .0413763 & .0389859 & 1.06 & 0.291 & -.0358545 & .1186071 \\
\hline year 1985 & .0098081 & .0320682 & 0.31 & 0.760 & -.0537186 & .0733349 \\
\hline year 1986 & .0666633 & .0444902 & 1.50 & 0.137 & -.0214714 & .154798 \\
\hline year_1987 & .0719674 & .0480215 & 1.50 & 0.137 & -.0231628 & .1670976 \\
\hline year 1988 & .0802089 & .0357808 & 2.24 & 0.027 & .0093273 & .1510905 \\
\hline year 1989 & .0538909 & .0473162 & 1.14 & 0.257 & -.0398421 & .1476238 \\
\hline year_1990 & -.0445709 & .0229706 & -1.94 & 0.055 & -.0900755 & .0009337 \\
\hline year_1991 & .0551381 & .0356181 & 1.55 & 0.124 & -.0154211 & .1256973 \\
\hline year 1992 & .0111847 & .0257153 & 0.43 & 0.664 & -.0397571 & .0621264 \\
\hline year 1993 & -.0022843 & .0256541 & -0.09 & 0.929 & -.0531048 & .0485362 \\
\hline year_1994 & .0508357 & .029263 & 1.74 & 0.085 & -.007134 & .1088054 \\
\hline year_1995 & -.0384342 & .0458349 & -0.84 & 0.403 & -.1292327 & .0523643 \\
\hline year_1996 & -.0457417 & .0357311 & -1.28 & 0.203 & -.1165248 & .0250413 \\
\hline year_1997 & .0036798 & .0301852 & 0.12 & 0.903 & -.0561168 & .0634765 \\
\hline year_1998 & -.017658 & .0337322 & -0.52 & 0.602 & -.0844813 & .0491653 \\
\hline year_1999 & -.0566496 & .0598262 & -0.95 & 0.346 & -.1751648 & .0618655 \\
\hline year_2000 & .0010919 & .0242738 & 0.04 & 0.964 & -.0469944 & .0491782 \\
\hline year 2001 & .0166393 & .0309446 & 0.54 & 0.592 & -.0446617 & .0779403 \\
\hline year 2002 & .0143897 & .0278177 & 0.52 & 0.606 & -.0407169 & .0694963 \\
\hline year 2003 & -.0226475 & .0345032 & -0.66 & 0.513 & -.090998 & .045703 \\
\hline $\operatorname{may} 1631$ & .0558081 & .0193472 & 2.88 & 0.005 & .0174814 & .0941347 \\
\hline jun115 & .1328544 & .0155225 & 8.56 & 0.000 & .1021045 & .1636043 \\
\hline jun 1630 & .2007011 & .0203845 & 9.85 & 0.000 & .1603195 & .2410827 \\
\hline jul115 & .2774454 & .0197493 & 14.05 & 0.000 & .2383223 & .3165685 \\
\hline jul1631 & .3154938 & .0230649 & 13.68 & 0.000 & .2698023 & .3611852 \\
\hline $\operatorname{aug} 115$ & .3081037 & .0226207 & 13.62 & 0.000 & .2632923 & .3529152 \\
\hline cp_aledoil & .0032036 & .0108706 & 0.29 & 0.769 & -.018331 & .0247381 \\
\hline gdd_aledoil & -.0005093 & .0007324 & -0.70 & 0.488 & -.0019602 & .0009416 \\
\hline cp2_aledoil & -.0007615 & .0012479 & -0.61 & 0.543 & -.0032336 & .0017106 \\
\hline gdd2_aledoil & $-3.82 e-06$ & $4.58 e-06$ & -0.83 & 0.406 & -.0000129 & $5.25 e-06$ \\
\hline cpgdd_aled l & $-6.57 e-06$ & .0001365 & -0.05 & 0.962 & -.0002769 & .0002638 \\
\hline _cons & .4669736 & .027856 & 16.76 & 0.000 & .411791 & .5221561 \\
\hline
\end{tabular}




\title{
Morrisonville, IL
}

\author{
Linear regression
}

$\begin{array}{llr}\text { Number of obs } & & 154 \\ \text { F }(32, \text { 121) } & 33.55 \\ \text { Prob F } & =0000 \\ \text { R-squared } & =0.8947 \\ \text { Root MSE } & =.07398\end{array}$

\begin{tabular}{|c|c|c|c|c|c|c|}
\hline ndvi_morri l & Coef. & $\begin{array}{c}\text { Robust } \mathrm{HC} 3 \\
\text { Std. Err. }\end{array}$ & $t$ & $P>|t|$ & [95\% Conf. & Interval] \\
\hline year 1983 & - 0811253 & 0506382 & $-1=60$ & 0112 & 181377 & 0191264 \\
\hline year 1984 & .0429293 & .0461077 & 0.93 & 0.354 & $\begin{array}{r}-.181377 \\
-.0483531\end{array}$ & $\begin{array}{l}.0191264 \\
.1342117\end{array}$ \\
\hline year 1985 & .0143696 & .0357423 & 0.40 & 0.688 & -.0563917 & .085131 \\
\hline year_1986 & .0495223 & .0496923 & 1.00 & 0.321 & -.0488567 & .1479014 \\
\hline year_1987 & .01906 & .0489011 & 0.39 & 0.697 & -.0777526 & .1158726 \\
\hline year_1988 & .0137417 & .0415844 & 0.33 & 0.742 & -.0685856 & .096069 \\
\hline year_1989 & .0229432 & .0510319 & 0.45 & 0.654 & -.078088 & .1239744 \\
\hline year 1990 & -.0330794 & .0363546 & -0.91 & 0.365 & -.105053 & .0388942 \\
\hline year_1991 & .024442 & .0427706 & 0.57 & 0.569 & -.0602338 & .1091177 \\
\hline year_1992 & -.0042098 & .0402397 & -0.10 & 0.917 & -.0838748 & .0754552 \\
\hline year 1993 & -.0091369 & .0420075 & -0.22 & 0.828 & -.0923019 & .0740281 \\
\hline year_1994 & .0517123 & .0360045 & 1.44 & 0.154 & -.0195682 & .1229927 \\
\hline year_1995 & -.0906695 & .0590581 & -1.54 & 0.127 & -.2075906 & .0262516 \\
\hline year_1996 & -.0052172 & .041094 & -0.13 & 0.899 & -.0865735 & .0761392 \\
\hline year_1997 & -.010958 & .0437351 & -0.25 & 0.803 & -.0975431 & .0756271 \\
\hline year_1998 & -.0479726 & .0394084 & -1.22 & 0.226 & -.1259919 & .0300467 \\
\hline year_1999 & -.0286223 & .0443692 & -0.65 & 0.520 & -.1164629 & .0592183 \\
\hline year_2000 & .0313374 & .0456973 & 0.69 & 0.494 & -.0591323 & .1218072 \\
\hline year_2001 & .0333922 & .0365964 & 0.91 & 0.363 & -.0390601 & .1058444 \\
\hline year_2002 & -.0603881 & .0467445 & -1.29 & 0.199 & -.1529312 & .0321549 \\
\hline year_2003 & -.0301362 & .0496677 & -0.61 & 0.545 & -.1284665 & .0681941 \\
\hline $\operatorname{may} 1631$ & .0595839 & .0247821 & 2.40 & 0.018 & .0105212 & .1086466 \\
\hline jun 115 & .1605598 & .0245791 & 6.53 & 0.000 & .1118989 & .2092206 \\
\hline jun 1630 & .2803748 & .0287707 & 9.75 & 0.000 & .2234157 & .337334 \\
\hline jul115 & .4232788 & .0247154 & 17.13 & 0.000 & .3743481 & .4722094 \\
\hline jul1631 & .4832877 & .0226614 & 21.33 & 0.000 & .4384235 & .5281519 \\
\hline $\operatorname{aug} 115$ & .4806842 & .0233544 & 20.58 & 0.000 & .434448 & .5269204 \\
\hline cp_morriso l & -.0011769 & .0118374 & -0.10 & 0.921 & -.0246122 & .0222584 \\
\hline dd_morris 1 & -.0357936 & .0355391 & -1.01 & 0.316 & -.1061527 & .0345654 \\
\hline morris l & .000217 & .0017368 & 0.12 & 0.901 & -.0032215 & .0036554 \\
\hline $\mathrm{d} \overline{2}$ morri l & .004254 & .0042252 & 1.01 & 0.316 & -.004111 & .0126189 \\
\hline gdd__morr 1 & .0128155 & .0103021 & 1.24 & 0.216 & -.0075803 & .0332113 \\
\hline cons & .3696421 & .0398862 & 9.27 & 0.000 & .2906767 & .4486074 \\
\hline
\end{tabular}




\section{Windsor, IL}

Linear regression

$\begin{array}{llr}\text { Number of obs } & = & 152 \\ \mathrm{~F}(32, \text { 119) } & =23.57 \\ \text { Prob }>\text { F } & 0.0000 \\ \text { R-squared } & =0.8429 \\ \text { Root MSE } & = & .07294\end{array}$

\begin{tabular}{|c|c|c|c|c|c|c|}
\hline ndvi_winds 1 & Coef. & $\begin{array}{l}\text { Robust } \mathrm{HC} 3 \\
\text { Std. Err. }\end{array}$ & $t$ & $P>|t|$ & [95\% Conf. & Int $\epsilon$ \\
\hline year_1983 & -.0133347 & .0326037 & -0.41 & 0.683 & -.0778934 & .051224 \\
\hline year_1984 & -.0278832 & .0498616 & -0.56 & 0.577 & -.1266142 & .0708479 \\
\hline year_1985 & .1015616 & .0306196 & 3.32 & 0.001 & .0409318 & .1621914 \\
\hline year_1986 & .0653765 & .0497819 & 1.31 & 0.192 & -.0331967 & .1639496 \\
\hline year_1987 & .0672948 & .0327846 & 2.05 & 0.042 & .0023781 & .1322115 \\
\hline year_1988 & .0003337 & .0578562 & 0.01 & 0.995 & -.1142273 & .1148946 \\
\hline year_1989 & -.0562159 & .042935 & -1.31 & 0.193 & -.1412315 & .0287997 \\
\hline year_1990 & -.0123381 & .0288918 & -0.43 & 0.670 & -.0695467 & .0448705 \\
\hline year-1991 & .0403691 & .0354267 & 1.14 & 0.257 & -.0297792 & .1105174 \\
\hline year_1992 & .076587 & .0288837 & 2.65 & 0.009 & .0193944 & .1337797 \\
\hline year_1993 & .0704206 & .0331111 & 2.13 & 0.036 & .0048573 & .135984 \\
\hline year_1994 & .0666185 & .0266063 & 2.50 & 0.014 & .0139354 & .1193016 \\
\hline year_1995 & .0240091 & .0378707 & 0.63 & 0.527 & -.0509787 & .0989969 \\
\hline year_1996 & -.0487952 & .0293252 & -1.66 & 0.099 & -.106862 & .0092715 \\
\hline year_1997 & .0477792 & .0345926 & 1.38 & 0.170 & -.0207177 & .116276 \\
\hline year_1998 & -.0405767 & .0418804 & -0.97 & 0.335 & -.1235041 & .0423507 \\
\hline year-1999 & -.0368495 & .0596437 & -0.62 & 0.538 & -.15495 & .0812509 \\
\hline year_2000 & .0778095 & .032192 & 2.42 & 0.017 & .0140661 & .1415529 \\
\hline year_2001 & .0596248 & .0412822 & 1.44 & 0.151 & -.0221181 & .1413677 \\
\hline year_2002 & -.0538251 & .0553631 & -0.97 & 0.333 & -.1634496 & .0557994 \\
\hline year_2003 & .023911 & .0527589 & 0.45 & 0.651 & -.0805568 & .1283788 \\
\hline maȳ1631 & .0685738 & .0282825 & 2.42 & 0.017 & .0125715 & .1245761 \\
\hline jun 115 & .1484165 & .0256191 & 5.79 & 0.000 & .097688 & .1991449 \\
\hline jun 1630 & .2278831 & .0297652 & 7.66 & 0.000 & .168945 & .2868212 \\
\hline jul115 & .3176406 & .0293382 & 10.83 & 0.000 & .2595481 & .3757331 \\
\hline jul1631 & .3730634 & .0296399 & 12.59 & 0.000 & .3143735 & .4317533 \\
\hline $\operatorname{aug} 115$ & .3737261 & .0266637 & 14.02 & 0.000 & .3209292 & .4265229 \\
\hline indsoril & .0011287 & .015059 & 0.07 & 0.940 & -.0286896 & .030947 \\
\hline gdd__windso l & -.0001142 & .0010117 & -0.11 & 0.910 & -.0021175 & .0018891 \\
\hline cp2_windso 1 & -.0004187 & .0020536 & -0.20 & 0.839 & -.0044849 & .0036476 \\
\hline gdd 2 _winds 1 & $3.55 e-06$ & $7.41 e-06$ & 0.48 & 0.633 & -.0000111 & .0000182 \\
\hline 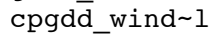 & -.0000677 & .000192 & -0.35 & 0.725 & -.0004479 & .0003125 \\
\hline cons & .4027587 & .0342261 & 11.77 & 0.000 & .3349875 & .4705298 \\
\hline
\end{tabular}




\section{Whitehall, IL}

Linear regression

$\begin{array}{llr}\text { Number of obs } & = & 154 \\ \mathrm{~F}(32, \text { 121) } & 16.17 \\ \text { Prob }>\text { F } & 0.0000 \\ \text { R-squared } & =0.8223 \\ \text { Root MSE } & = & .05041\end{array}$

\begin{tabular}{|c|c|c|c|c|c|c|}
\hline ndvi_white 1 & Coef. & $\begin{array}{c}\text { Robust HC3 } \\
\text { Std. Err. }\end{array}$ & $t$ & $P>|t|$ & {$[95 \%$ Conf } & Inte \\
\hline year_1983 & .0223346 & .0335438 & 0.67 & 0.507 & -.0440743 & .0887434 \\
\hline year_1984 & -.0406866 & .0389768 & -1.04 & 0.299 & -.1178513 & .0364782 \\
\hline year_1985 & -.0312302 & .0295607 & -1.06 & 0.293 & -.0897535 & .0272931 \\
\hline year_1986 & .0058827 & .0391893 & 0.15 & 0.881 & -.0717028 & .0834682 \\
\hline year_1987 & -.0144346 & .0269859 & -0.53 & 0.594 & -.0678603 & .0389911 \\
\hline year_1988 & -.0349025 & .0303447 & -1.15 & 0.252 & -.0949778 & .0251728 \\
\hline year_1989 & -.0844149 & .0327922 & -2.57 & 0.011 & -.1493357 & -.0194942 \\
\hline year_1990 & -.0352763 & .0308899 & -1.14 & 0.256 & -.096431 & .0258783 \\
\hline year_1991 & -.0261203 & .0271642 & -0.96 & 0.338 & -.0798989 & .0276583 \\
\hline year_1992 & -.0811632 & .0323956 & -2.51 & 0.014 & -.1452989 & -.0170276 \\
\hline year_1993 & .021119 & .0388194 & 0.54 & 0.587 & -.0557342 & .0979722 \\
\hline year_1994 & -.0237974 & .0294549 & -0.81 & 0.421 & -.0821111 & .0345163 \\
\hline year_1995 & -.0878394 & .0409082 & -2.15 & 0.034 & -.1688279 & -.0068508 \\
\hline year_1996 & -.0170933 & .0317738 & -0.54 & 0.592 & -.079998 & .0458114 \\
\hline year_1997 & -.044292 & .0281482 & -1.57 & 0.118 & -.1000187 & .0114348 \\
\hline year_1998 & -.0545219 & .0338591 & -1.61 & 0.110 & -.121555 & .0125111 \\
\hline year_1999 & -.0901575 & .0445167 & -2.03 & 0.045 & -.17829 & -.0020251 \\
\hline year_2000 & -.0183657 & .0321085 & -0.57 & 0.568 & -.0819329 & .0452016 \\
\hline year_2001 & -.0503882 & .0281932 & -1.79 & 0.076 & -.106204 & .0054277 \\
\hline year_2002 & -.0929357 & .0291355 & -3.19 & 0.002 & -.1506171 & -.0352543 \\
\hline year_2003 & -.0287054 & .0276783 & -1.04 & 0.302 & -.0835019 & .026091 \\
\hline $\operatorname{may} 1631$ & .04801 & .0192532 & 2.49 & 0.014 & .0098932 & .0861269 \\
\hline jun115 & .085768 & .0182012 & 4.71 & 0.000 & .049734 & .121802 \\
\hline jun 1630 & .1496914 & .0175059 & 8.55 & 0.000 & .1150338 & .184349 \\
\hline jul115 & .2200903 & .0180048 & 12.22 & 0.000 & .184445 & .2557355 \\
\hline jul1631 & .2444999 & .0190758 & 12.82 & 0.000 & .2067342 & .2822655 \\
\hline $\operatorname{aug} 115$ & .2522601 & .0177123 & 14.24 & 0.000 & .217194 & .2873262 \\
\hline hiteha l & -.0182543 & .0117523 & -1.55 & 0.123 & -.0415211 & .0050124 \\
\hline gdd__whiteh l & -.001681 & .0013968 & -1.20 & 0.231 & -.0044463 & .0010844 \\
\hline cp2_whiteh 1 & .0034162 & .0021873 & 1.56 & 0.121 & -.0009141 & .0077466 \\
\hline gdd 2 _white 1 & $4.35 e-06$ & $8.25 e-06$ & 0.53 & 0.599 & -.000012 & .0000207 \\
\hline ogdd__whit l & .0004228 & .0004887 & 0.87 & 0.389 & -.0005447 & .0013904 \\
\hline _cons & .573954 & .028187 & 20.36 & 0.000 & .5181505 & .6297576 \\
\hline
\end{tabular}




\section{Beaverdam, KY}

Linear regression

$\begin{array}{llr}\text { Number of obs } & = & 154 \\ \mathrm{~F}(32, \text { 121) } & = & 1.85 \\ \text { Prob }>\text { F } & =0092 \\ \text { R-squared } & =0.3194 \\ \text { Root MSE } & = & .06148\end{array}$

\begin{tabular}{|c|c|c|c|c|c|c|}
\hline ndvi_beave $y$ & Coef. & $\begin{array}{l}\text { Robust } \mathrm{HC} 3 \\
\text { Std. Err. }\end{array}$ & $t$ & $P>|t|$ & [95\% Conf. & Interval] \\
\hline year_1983 & -.0118609 & .0297693 & -0.40 & 0.691 & -.070797 & .0470752 \\
\hline year_1984 & .0038685 & .0277082 & 0.14 & 0.889 & -.0509872 & .0587242 \\
\hline year_1985 & -.004731 & .0401411 & -0.12 & 0.906 & -.084201 & .074739 \\
\hline year_1986 & -.0181012 & .0449297 & -0.40 & 0.688 & -.1070514 & .070849 \\
\hline year_1987 & .0065834 & .0363066 & 0.18 & 0.856 & -.0652951 & .0784619 \\
\hline year_1988 & -.0276831 & .0345024 & -0.80 & 0.424 & -.0959898 & .0406235 \\
\hline year_1989 & .0424528 & .0268849 & 1.58 & 0.117 & -.010773 & .0956786 \\
\hline year_1990 & .0284982 & .0329006 & 0.87 & 0.388 & -.0366373 & .0936337 \\
\hline year_1991 & -.0027505 & .0285681 & -0.10 & 0.923 & -.0593085 & .0538076 \\
\hline year_1992 & .0125325 & .034507 & 0.36 & 0.717 & -.0557832 & .0808483 \\
\hline year_1993 & .0453328 & .0294578 & 1.54 & 0.126 & -.0129868 & .1036524 \\
\hline year_1994 & .0329448 & .0292241 & 1.13 & 0.262 & -.0249119 & .0908015 \\
\hline year_1995 & -.0130507 & .0273294 & -0.48 & 0.634 & -.0671565 & .0410552 \\
\hline year_1996 & -.0005167 & .03135 & -0.02 & 0.987 & -.0625823 & .0615488 \\
\hline year_1997 & -.0542115 & .0373412 & -1.45 & 0.149 & -.1281383 & .0197153 \\
\hline year_1998 & .0351133 & .0262696 & 1.34 & 0.184 & -.0168944 & .087121 \\
\hline year_1999 & -.0651472 & .0695365 & -0.94 & 0.351 & -.202813 & .0725186 \\
\hline year_2000 & .0287427 & .0243712 & 1.18 & 0.241 & -.0195065 & .0769918 \\
\hline year_2001 & -.0107115 & .0374518 & -0.29 & 0.775 & -.0848571 & .0634342 \\
\hline year_2002 & .0157513 & .0423629 & 0.37 & 0.711 & -.0681172 & .0996199 \\
\hline year_2003 & -.0145583 & .0320414 & -0.45 & 0.650 & -.0779928 & .0488761 \\
\hline maȳ1631 & .0074414 & .0234967 & 0.32 & 0.752 & -.0390765 & .0539594 \\
\hline jun115 & .0165654 & .0234309 & 0.71 & 0.481 & -.0298223 & .062953 \\
\hline jun 1630 & .0458894 & .0253981 & 1.81 & 0.073 & -.0043929 & .0961717 \\
\hline jul115 & .0581596 & .0323198 & 1.80 & 0.074 & -.005826 & .1221452 \\
\hline jul1631 & .0604035 & .025803 & 2.34 & 0.021 & .0093197 & .1114874 \\
\hline $\operatorname{aug} 115$ & .0572195 & .0242455 & 2.36 & 0.020 & .0092191 & .1052199 \\
\hline averd $\sim y$ & -.0064646 & .0177389 & -0.36 & 0.716 & -.0415834 & .0286542 \\
\hline gdd_beaver $\sim$ y & -.0004401 & .0007742 & -0.57 & 0.571 & -.0019727 & .0010926 \\
\hline cp2_beaver $\sim y$ & .0000682 & .0022253 & 0.03 & 0.976 & -.0043373 & .0044737 \\
\hline gdd 2 _beave $\sim y$ & $2.44 e-06$ & $4.68 e-06$ & 0.52 & 0.603 & $-6.83 e-06$ & .0000117 \\
\hline de__beav $\sim$ y & .0000672 & .0001512 & 0.44 & 0.658 & -.0002321 & .0003664 \\
\hline _cons & .6336632 & .0355898 & 17.80 & 0.000 & .5632038 & .7041225 \\
\hline
\end{tabular}




\section{Providence, KY}

Linear regression

$\begin{array}{llr}\text { Number of obs } & = & 152 \\ \mathrm{~F}(32, \text { 119) } & = & 4.36 \\ \text { Prob }>\text { F } & =0000 \\ \text { R-squared } & =0.4770 \\ \text { Root MSE } & = & .05054\end{array}$

\begin{tabular}{|c|c|c|c|c|c|c|}
\hline ndvi_provi y & Coef. & $\begin{array}{c}\text { Robust HC3 } \\
\text { Std. Err. }\end{array}$ & $t$ & $P>|t|$ & {$[95 \%$ Con } & Int \\
\hline year_1983 & -.0355821 & .0246205 & -1.45 & 0.151 & -.0843331 & .0131689 \\
\hline year_1984 & -.0685089 & .0224074 & -3.06 & 0.003 & -.1128778 & -.02414 \\
\hline year_1985 & -.0511872 & .0213744 & -2.39 & 0.018 & -.0935106 & -.0088638 \\
\hline year_1986 & -.0352316 & .0379043 & -0.93 & 0.355 & -.1102859 & .0398228 \\
\hline year_1987 & .0115914 & .0276409 & 0.42 & 0.676 & -.0431404 & .0663231 \\
\hline year_1988 & -.0119616 & .0304438 & -0.39 & 0.695 & -.0722432 & .0483201 \\
\hline year_1989 & -.056194 & .0201797 & -2.78 & 0.006 & -.0961519 & -.0162361 \\
\hline year_1990 & -.0104509 & .0238966 & -0.44 & 0.663 & -.0577686 & .0368668 \\
\hline year_1991 & -.0252115 & .0233096 & -1.08 & 0.282 & -.0713669 & .0209438 \\
\hline year_1992 & -.0339439 & .0252002 & -1.35 & 0.181 & -.0838428 & .0159549 \\
\hline year_1993 & -.0040339 & .0238378 & -0.17 & 0.866 & -.0512352 & .0431673 \\
\hline year_1994 & .0155524 & .0211186 & 0.74 & 0.463 & -.0262644 & .0573693 \\
\hline year_1995 & -.0190936 & .0280305 & -0.68 & 0.497 & -.0745968 & .0364097 \\
\hline year_1996 & -.0234261 & .0225682 & -1.04 & 0.301 & -.0681135 & .0212612 \\
\hline year_1997 & -.0357593 & .0306236 & -1.17 & 0.245 & -.096397 & .0248785 \\
\hline year_1998 & -.0049703 & .0248385 & -0.20 & 0.842 & -.054153 & .0442125 \\
\hline year_1999 & -.0505224 & .0357144 & -1.41 & 0.160 & -.1212405 & .0201956 \\
\hline year_2000 & .0123247 & .0278548 & 0.44 & 0.659 & -.0428306 & .0674799 \\
\hline year_2001 & -.0236032 & .0366831 & -0.64 & 0.521 & -.0962394 & .049033 \\
\hline year_2002 & -.014949 & .0517256 & -0.29 & 0.773 & -.1173709 & .0874728 \\
\hline year_2003 & -.0215267 & .0301102 & -0.71 & 0.476 & -.0811478 & .0380945 \\
\hline $\operatorname{may} 1631$ & .0331683 & .0195738 & 1.69 & 0.093 & -.0055898 & .0719264 \\
\hline jun115 & .0584579 & .0168899 & 3.46 & 0.001 & .0250143 & .0919015 \\
\hline jun 1630 & .0723926 & .0196543 & 3.68 & 0.000 & .0334752 & .1113101 \\
\hline jul115 & .0968744 & .0229735 & 4.22 & 0.000 & .0513845 & .1423643 \\
\hline jul1631 & .0939209 & .0279004 & 3.37 & 0.001 & .0386754 & .1491665 \\
\hline $\operatorname{aug} 115$ & .1087523 & .0194447 & 5.59 & 0.000 & .0702498 & .1472548 \\
\hline rovide y & -.0043347 & .0126924 & -0.34 & 0.733 & -.029467 & .0207976 \\
\hline gdd_provid y & -.000445 & .000372 & -1.20 & 0.234 & -.0011816 & .0002916 \\
\hline cp2_provid y & .0004866 & .0018536 & 0.26 & 0.793 & -.0031837 & .004157 \\
\hline gdd2__provi y & $1.27 e-06$ & $1.81 e-06$ & 0.70 & 0.484 & $-2.32 e-06$ & $4.86 e-06$ \\
\hline cpgdd__prov $\sim \mathrm{y}$ & .0001099 & .0000833 & 1.32 & 0.189 & -.000055 & .0002748 \\
\hline _cons & .6452699 & .0275488 & 23.42 & 0.000 & .5907205 & .6998193 \\
\hline
\end{tabular}




\section{Scottsville, KY}

Linear regression

$\begin{array}{llr}\text { Number of obs } & = & 154 \\ \mathrm{~F}(32, \text { 121) } & = & 1.70 \\ \text { Prob }>\text { F } & =0211 \\ \text { R-squared } & =0.2918 \\ \text { Root MSE } & = & .05517\end{array}$

\begin{tabular}{|c|c|c|c|c|c|c|}
\hline ndvi_scott $\sim y$ & Coef. & $\begin{array}{l}\text { Robust } \mathrm{HC} 3 \\
\text { Std. Err. }\end{array}$ & $t$ & $P>|t|$ & [95\% Conf. & Inte \\
\hline year_1983 & -.0104786 & .0267665 & -0.39 & 0.696 & -.0634699 & .0425126 \\
\hline year_1984 & .0126503 & .0240673 & 0.53 & 0.600 & -.0349972 & .0602978 \\
\hline year_1985 & -.0294144 & .0239934 & -1.23 & 0.223 & -.0769157 & .0180869 \\
\hline year_1986 & .0062355 & .042864 & 0.15 & 0.885 & -.0786251 & .091096 \\
\hline year_1987 & .0329745 & .0238171 & 1.38 & 0.169 & -.0141776 & .0801267 \\
\hline year_1988 & -.0568934 & .0366856 & -1.55 & 0.124 & -.1295221 & .0157354 \\
\hline year_1989 & -.0173505 & .0239443 & -0.72 & 0.470 & -.0647545 & .0300535 \\
\hline year_1990 & .0210701 & .0230661 & 0.91 & 0.363 & -.0245953 & .0667354 \\
\hline year_1991 & .0103841 & .0251802 & 0.41 & 0.681 & -.0394668 & .060235 \\
\hline year_1992 & .0259999 & .0246768 & 1.05 & 0.294 & -.0228544 & .0748542 \\
\hline year_1993 & .061082 & .0244326 & 2.50 & 0.014 & .0127111 & .1094529 \\
\hline year_1994 & .016381 & .0273819 & 0.60 & 0.551 & -.0378288 & .0705907 \\
\hline year_1995 & .0173408 & .0235254 & 0.74 & 0.462 & -.029234 & .0639156 \\
\hline year_1996 & .0391999 & .0271739 & 1.44 & 0.152 & -.0145979 & .0929977 \\
\hline year_1997 & -.049908 & .0519731 & -0.96 & 0.339 & -.1528025 & .0529866 \\
\hline year_1998 & .0440536 & .0226205 & 1.95 & 0.054 & -.0007298 & .0888369 \\
\hline year_1999 & -.044615 & .0294842 & -1.51 & 0.133 & -.1029868 & .0137568 \\
\hline year_2000 & -.0019378 & .0229541 & -0.08 & 0.933 & -.0473815 & .0435058 \\
\hline year_2001 & -.0304171 & .0282685 & -1.08 & 0.284 & -.086382 & .0255479 \\
\hline year_2002 & -.0130972 & .0406202 & -0.32 & 0.748 & -.0935157 & .0673212 \\
\hline year_2003 & -.0014389 & .0266493 & -0.05 & 0.957 & -.0541983 & .0513205 \\
\hline $\operatorname{may} 1631$ & .0008634 & .0202134 & 0.04 & 0.966 & -.0391544 & .0408813 \\
\hline jun115 & .0121209 & .0261908 & 0.46 & 0.644 & -.0397307 & .0639725 \\
\hline jun 1630 & .0124641 & .0198473 & 0.63 & 0.531 & -.0268289 & .051757 \\
\hline jul115 & -.0015421 & .0243684 & -0.06 & 0.950 & -.0497857 & .0467016 \\
\hline jul1631 & -.0069472 & .0221181 & -0.31 & 0.754 & -.0507357 & .0368414 \\
\hline $\operatorname{aug} 115$ & -.0077483 & .0230069 & -0.34 & 0.737 & -.0532966 & .0378 \\
\hline $\operatorname{cotts} v \sim \mathrm{y}$ & -.0169576 & .0111439 & -1.52 & 0.131 & -.0390199 & .0051047 \\
\hline gdd__scotts $\sim y$ & .0000891 & .0008371 & 0.11 & 0.915 & -.0015681 & .0017463 \\
\hline cp2_scotts $\sim y$ & .0021163 & .0014794 & 1.43 & 0.155 & -.0008126 & .0050452 \\
\hline gdd $\overline{2} \_s \cot t \sim y$ & $-1.05 e-06$ & $6.50 e-06$ & -0.16 & 0.872 & -.0000139 & .0000118 \\
\hline $\mathrm{d} \overline{\mathrm{d}} \_\mathrm{scot} \sim \mathrm{y}$ & -.0000128 & .0001295 & -0.10 & 0.922 & -.000269 & .0002435 \\
\hline cons & .7405423 & .0274051 & 27.02 & 0.000 & .6862866 & .7947981 \\
\hline
\end{tabular}


Farmville, VA

Linear regression

$\begin{array}{llr}\text { Number of obs } & = & 154 \\ \text { F }(32,121) & 2.18 \\ \text { Prob }>\text { F } & =0013 \\ \text { R-squared } & =0.2818 \\ \text { Root MSE } & = & .05002\end{array}$

\begin{tabular}{|c|c|c|c|c|c|c|}
\hline ndvi_farmv a & Coef. & $\begin{array}{c}\text { Robust HC3 } \\
\text { Std. Err. }\end{array}$ & $t$ & $P>|t|$ & [95\% Conf. & Interval] \\
\hline year_1983 & -.0072645 & .0336235 & -0.22 & 0.829 & -.0738309 & .059302 \\
\hline year_1984 & -.0237011 & .0284176 & -0.83 & 0.406 & -.0799611 & .032559 \\
\hline year_1985 & .0190816 & .0264131 & 0.72 & 0.471 & -.0332101 & .0713733 \\
\hline year_1986 & -.0528567 & .0366788 & -1.44 & 0.152 & -.1254721 & .0197586 \\
\hline year_1987 & -.0640799 & .0294799 & -2.17 & 0.032 & -.1224431 & -.0057168 \\
\hline year_1988 & .0045946 & .0268448 & 0.17 & 0.864 & -.0485518 & .057741 \\
\hline year_1989 & -.0072792 & .0314316 & -0.23 & 0.817 & -.0695063 & .0549478 \\
\hline year_1990 & -.0115152 & .0358936 & -0.32 & 0.749 & -.0825761 & .0595456 \\
\hline year_1991 & .0048707 & .0314733 & 0.15 & 0.877 & -.057439 & .0671804 \\
\hline year_1992 & -.0020645 & .0325448 & -0.06 & 0.950 & -.0664956 & .0623666 \\
\hline year_1993 & -.0396591 & .026318 & -1.51 & 0.134 & -.0917626 & .0124443 \\
\hline year_1994 & -.0048698 & .0257201 & -0.19 & 0.850 & -.0557895 & .04605 \\
\hline year_1995 & -.025707 & .0449763 & -0.57 & 0.569 & -.1147495 & .0633355 \\
\hline year_1996 & .0571876 & .0266907 & 2.14 & 0.034 & .0043462 & .1100289 \\
\hline year_1997 & .0156052 & .026884 & 0.58 & 0.563 & -.0376188 & .0688292 \\
\hline year_1998 & .0189363 & .0275089 & 0.69 & 0.493 & -.0355247 & .0733973 \\
\hline year_1999 & -.0461295 & .0285558 & -1.62 & 0.109 & -.1026632 & .0104042 \\
\hline year_2000 & -.0355375 & .0333357 & -1.07 & 0.289 & -.1015343 & .0304592 \\
\hline year_2001 & -.0011939 & .0329124 & -0.04 & 0.971 & -.0663527 & .063965 \\
\hline year_2002 & .0112215 & .0278006 & 0.40 & 0.687 & -.0438171 & .0662601 \\
\hline year_2003 & -.0258688 & .0310858 & -0.83 & 0.407 & -.0874114 & .0356737 \\
\hline $\operatorname{maȳ} 1631$ & .0168171 & .0145039 & 1.16 & 0.249 & -.0118972 & .0455314 \\
\hline jun115 & .0101518 & .0146879 & 0.69 & 0.491 & -.0189267 & .0392304 \\
\hline jun1630 & -.0082997 & .020464 & -0.41 & 0.686 & -.0488135 & .0322142 \\
\hline jul115 & .0098607 & .0142093 & 0.69 & 0.489 & -.0182704 & .0379918 \\
\hline jul1631 & -.0117791 & .0160646 & -0.73 & 0.465 & -.0435833 & .020025 \\
\hline $\operatorname{aug} 115$ & -.0143991 & .0143369 & -1.00 & 0.317 & -.0427828 & .0139847 \\
\hline cp_farmvil a & -.0093549 & .0111658 & -0.84 & 0.404 & -.0314605 & .0127508 \\
\hline gdd__farmvi a & .0002666 & .000882 & 0.30 & 0.763 & -.0014795 & .0020127 \\
\hline cp2_farmvi a & .0013016 & .0021463 & 0.61 & 0.545 & -.0029475 & .0055508 \\
\hline gdd 2 _farmv a & $-1.22 e-06$ & $5.58 e-06$ & -0.22 & 0.827 & -.0000123 & $9.82 e-06$ \\
\hline cpgdd__farm a & .0000436 & .0001482 & 0.29 & 0.769 & -.0002499 & .0003371 \\
\hline cons & .7553327 & .0269563 & 28.02 & 0.000 & .7019656 & .8086999 \\
\hline
\end{tabular}


Halfway, OR

Linear regression

$\begin{array}{llr}\text { Number of obs } & & 154 \\ \mathrm{~F}(32, \text { 121) } & 5.75 \\ \text { Prob }>\text { F } & 0.0000 \\ \text { R-squared } & =0.6129 \\ \text { Root MSE } & = & .05843\end{array}$

\begin{tabular}{|c|c|c|c|c|c|c|}
\hline ndvi_halfw r & Coef. & $\begin{array}{l}\text { Robust } \mathrm{HC} 3 \\
\text { Std. Err. }\end{array}$ & $t$ & $P>|t|$ & {$[95 \%$} & Int \\
\hline year 1983 & -.0045855 & .0273861 & -0.17 & 0.867 & -.0588036 & .0496326 \\
\hline year_1984 & -.1118311 & .0512977 & -2.18 & 0.031 & -.2133885 & -.0102737 \\
\hline year_1985 & -.0475247 & .0280116 & -1.70 & 0.092 & -.1029811 & .0079317 \\
\hline year_1986 & -.0363448 & .0317056 & -1.15 & 0.254 & -.0991144 & .0264247 \\
\hline year_1987 & -.0942497 & .0322533 & -2.92 & 0.004 & -.1581036 & -.0303957 \\
\hline year_1988 & -.0572674 & .0281454 & -2.03 & 0.044 & -.1129886 & -.0015463 \\
\hline year_1989 & -.1667029 & .0581581 & -2.87 & 0.005 & -.2818422 & -.0515637 \\
\hline year_1990 & .0204923 & .033272 & 0.62 & 0.539 & -.0453785 & .0863631 \\
\hline year_1991 & .0048531 & .0293357 & 0.17 & 0.869 & -.0532247 & .0629308 \\
\hline year_1992 & -.0216448 & .0268389 & -0.81 & 0.422 & -.0747795 & .0314898 \\
\hline year_1993 & .0366908 & .0333568 & 1.10 & 0.274 & -.0293478 & .1027294 \\
\hline year_1994 & -.0127621 & .0363253 & -0.35 & 0.726 & -.0846776 & .0591535 \\
\hline year_1995 & -.0055507 & .0248292 & -0.22 & 0.823 & -.0547067 & .0436052 \\
\hline year_1996 & -.0209193 & .0300682 & -0.70 & 0.488 & -.0804472 & .0386086 \\
\hline year_1997 & .0294366 & .0259497 & 1.13 & 0.259 & -.0219376 & .0808108 \\
\hline year_1998 & .0238996 & .0267826 & 0.89 & 0.374 & -.0291237 & .0769228 \\
\hline year_1999 & -.1262148 & .0546211 & -2.31 & 0.023 & -.2343518 & -.0180779 \\
\hline year_2000 & -.0332195 & .0309595 & -1.07 & 0.285 & -.0945121 & .0280731 \\
\hline year_2001 & -.0312373 & .0360201 & -0.87 & 0.388 & -.1025486 & .0400741 \\
\hline year_2002 & -.056997 & .0319156 & -1.79 & 0.077 & -.1201824 & .0061883 \\
\hline year_2003 & -.0370086 & .0407022 & -0.91 & 0.365 & -.1175894 & .0435722 \\
\hline $\operatorname{may} 1631$ & .0546615 & .0236037 & 2.32 & 0.022 & .0079317 & .1013914 \\
\hline jun115 & .1014561 & .0222314 & 4.56 & 0.000 & .0574431 & .1454691 \\
\hline jun 1630 & .121641 & .0239723 & 5.07 & 0.000 & .0741815 & .1691004 \\
\hline jul115 & .1248396 & .0258856 & 4.82 & 0.000 & .0735921 & .176087 \\
\hline jul1631 & .103763 & .0273066 & 3.80 & 0.000 & .0497025 & .1578236 \\
\hline $\operatorname{aug} 115$ & .0622505 & .0294709 & 2.11 & 0.037 & .003905 & .120596 \\
\hline alfwayor & -.0116825 & .0383896 & -0.30 & 0.761 & -.0876849 & .0643199 \\
\hline gdd_halfwa r & .0000197 & .0005368 & 0.04 & 0.971 & -.0010432 & .0010825 \\
\hline cp2_halfwa r & .002884 & .016586 & 0.17 & 0.862 & -.0299523 & .0357203 \\
\hline gdd 2 _halfw r & $-2.56 e-06$ & $2.42 e-06$ & -1.06 & 0.293 & $-7.36 e-06$ & $2.24 \mathrm{e}-06$ \\
\hline gdd__half r & .0002786 & .0005807 & 0.48 & 0.632 & -.000871 & .0014282 \\
\hline _cons & .6256836 & .0323239 & 19.36 & 0.000 & .56169 & .6896773 \\
\hline
\end{tabular}




\section{Heppner, OR}

Linear regression

$\begin{array}{llr}\text { Number of obs } & = & 154 \\ \mathrm{~F}(32, \text { 121) } & 17.49 \\ \text { Prob }>\text { F } & 0.0000 \\ \text { R-squared } & =0.8128 \\ \text { Root MSE } & = & .02952\end{array}$

\begin{tabular}{|c|c|c|c|c|c|c|}
\hline ndvi_heppn r & Coef. & $\begin{array}{l}\text { Robust } \mathrm{HC} 3 \\
\text { Std. Err. }\end{array}$ & $t$ & $P>|t|$ & {$[95 \%$ Con } & Int $\epsilon$ \\
\hline year_1983 & .0104216 & .0108217 & 0.96 & 0.337 & -.0110029 & .0318461 \\
\hline year_1984 & .0730094 & .015741 & 4.64 & 0.000 & .0418459 & .1041728 \\
\hline year_1985 & -.0109806 & .0146801 & -0.75 & 0.456 & -.0400437 & .0180826 \\
\hline year_1986 & .0023427 & .0116159 & 0.20 & 0.841 & -.0206541 & .0253395 \\
\hline year_1987 & .0150334 & .0224459 & 0.67 & 0.504 & -.0294041 & .0594709 \\
\hline year_1988 & .0337379 & .0158461 & 2.13 & 0.035 & .0023664 & .0651094 \\
\hline year_1989 & .0037682 & .0127102 & 0.30 & 0.767 & -.0213949 & .0289314 \\
\hline year_1990 & -.0299778 & .0131029 & -2.29 & 0.024 & -.0559184 & -.0040371 \\
\hline year_1991 & -.0035282 & .012817 & -0.28 & 0.784 & -.0289028 & .0218465 \\
\hline year_1992 & -.0173372 & .0149962 & -1.16 & 0.250 & -.0470262 & .0123517 \\
\hline year 1993 & .0538795 & .0103431 & 5.21 & 0.000 & .0334025 & .0743565 \\
\hline year_1994 & -.0144165 & .014936 & -0.97 & 0.336 & -.0439863 & .0151534 \\
\hline year_1995 & .0475247 & .0123802 & 3.84 & 0.000 & .0230148 & .0720347 \\
\hline year_1996 & .0199534 & .0088575 & 2.25 & 0.026 & .0024176 & .0374892 \\
\hline year_1997 & .0158352 & .0181152 & 0.87 & 0.384 & -.0200286 & .0516991 \\
\hline year_1998 & .005315 & .0144582 & 0.37 & 0.714 & -.0233088 & .0339389 \\
\hline year_1999 & -.0943604 & .0267755 & -3.52 & 0.001 & -.1473696 & -.0413512 \\
\hline year_2000 & .0171573 & .0173318 & 0.99 & 0.324 & -.0171556 & .0514701 \\
\hline year_2001 & .0041758 & .0150813 & 0.28 & 0.782 & -.0256817 & .0340333 \\
\hline year_2002 & -.0568254 & .013156 & -4.32 & 0.000 & -.0828711 & -.0307797 \\
\hline year_2003 & -.0029935 & .0153802 & -0.19 & 0.846 & -.0334427 & .0274557 \\
\hline maȳ1631 & .0045386 & .0102699 & 0.44 & 0.659 & -.0157933 & .0248705 \\
\hline jun115 & -.0186404 & .010515 & -1.77 & 0.079 & -.0394577 & .0021768 \\
\hline jun 1630 & -.0367275 & .0110949 & -3.31 & 0.001 & -.0586928 & -.0147622 \\
\hline jul115 & -.07193 & .0104631 & -6.87 & 0.000 & -.0926446 & -.0512155 \\
\hline jul1631 & -.1005427 & .0105175 & -9.56 & 0.000 & -.1213649 & -.0797205 \\
\hline $\operatorname{aug} 115$ & -.127 & .0101061 & -12.57 & 0.000 & -.1470077 & -.1069923 \\
\hline eppneror & -.0002919 & .0156138 & -0.02 & 0.985 & -.0312036 & .0306197 \\
\hline gd"̄_heppne r & .0010318 & .001987 & 0.52 & 0.605 & -.0029019 & .0049656 \\
\hline cp2_heppne r & -.0078946 & .0064562 & -1.22 & 0.224 & -.0206764 & .0048872 \\
\hline dd 2 _heppn r & -.0000477 & .000089 & -0.54 & 0.593 & -.0002239 & .0001284 \\
\hline $\bar{d}$ d_hepp r & -.0051527 & .0054849 & -0.94 & 0.349 & -.0160114 & .0057061 \\
\hline Cons & .386386 & .0122575 & 31.52 & 0.000 & .362119 & .4106529 \\
\hline
\end{tabular}




\section{Vernonia, OR}

Linear regression

$\begin{array}{llr}\text { Number of obs } & = & 154 \\ \mathrm{~F}(29,124) & 5.72 \\ \text { Prob }>\text { F } & =00000 \\ \text { R-squared } & =0.6213 \\ \text { Root MSE } & = & .04315\end{array}$

\begin{tabular}{|c|c|c|c|c|c|c|}
\hline ndvi_verno r & Coef. & $\begin{array}{l}\text { Robust } \mathrm{HC} 3 \\
\text { Std. Err. }\end{array}$ & $t$ & $P>|t|$ & [95\% Conf. & Interval] \\
\hline year_1983 & .0012868 & .0223519 & 0.06 & 0.954 & -.0429539 & .0455276 \\
\hline year_1984 & .0193084 & .0272858 & 0.71 & 0.480 & -.0346979 & .0733147 \\
\hline year_1985 & -.0037914 & .0137172 & -0.28 & 0.783 & -.0309416 & .0233589 \\
\hline year_1986 & -.0462867 & .0220618 & -2.10 & 0.038 & -.0899532 & -.0026201 \\
\hline year_1987 & -.0085904 & .0169038 & -0.51 & 0.612 & -.0420478 & .024867 \\
\hline year_1988 & .0041859 & .0129849 & 0.32 & 0.748 & -.0215148 & .0298866 \\
\hline year_1989 & .0245919 & .0116997 & 2.10 & 0.038 & .0014349 & .047749 \\
\hline year_1990 & -.0092312 & .014589 & -0.63 & 0.528 & -.038107 & .0196445 \\
\hline year_1991 & -.0358473 & .0157438 & -2.28 & 0.025 & -.0670087 & -.0046858 \\
\hline year_1992 & -.0092113 & .0141861 & -0.65 & 0.517 & -.0372895 & .0188668 \\
\hline year_1993 & .0350784 & .0229814 & 1.53 & 0.129 & -.0104082 & .080565 \\
\hline year_1994 & -.0047755 & .0248134 & -0.19 & 0.848 & -.0538881 & .0443372 \\
\hline year_1995 & -.0029645 & .012271 & -0.24 & 0.810 & -.0272523 & .0213233 \\
\hline year_1996 & .003398 & .0174869 & 0.19 & 0.846 & -.0312134 & .0380094 \\
\hline year_1997 & .0465179 & .0166976 & 2.79 & 0.006 & .0134687 & .0795672 \\
\hline year_1998 & -.0124099 & .0150768 & -0.82 & 0.412 & -.0422511 & .0174314 \\
\hline year_1999 & -.1170521 & .0567742 & -2.06 & 0.041 & -.2294241 & -.0046801 \\
\hline year_2000 & -.002864 & .0145068 & -0.20 & 0.844 & -.031577 & .0258491 \\
\hline year_2001 & -.032492 & .0143423 & -2.27 & 0.025 & -.0608794 & -.0041047 \\
\hline year_2002 & .003871 & .0133022 & 0.29 & 0.772 & -.0224577 & .0301997 \\
\hline year_2003 & -.0262239 & .0194679 & -1.35 & 0.180 & -.0647563 & .0123084 \\
\hline $\operatorname{may} 1631$ & .0462553 & .0159315 & 2.90 & 0.004 & .0147224 & .0777882 \\
\hline jun115 & .0587845 & .0146515 & 4.01 & 0.000 & .0297851 & .087784 \\
\hline jun 1630 & .0697777 & .0191124 & 3.65 & 0.000 & .0319489 & .1076066 \\
\hline jul115 & .0762828 & .0170133 & 4.48 & 0.000 & .0426087 & .1099568 \\
\hline jul1631 & .0832142 & .0158694 & 5.24 & 0.000 & .0518041 & .1146243 \\
\hline $\operatorname{aug} 115$ & .0770531 & .0141614 & 5.44 & 0.000 & .0490237 & .1050825 \\
\hline $\begin{array}{l}\text { ernoni r } \\
\text { vernon } \sim r\end{array}$ & $\begin{array}{c}-.0652368 \\
\text { (dropped) }\end{array}$ & .0158303 & $-4 \cdot 12$ & 0.000 & -.0965694 & -.0339041 \\
\hline cp2_vernon $\sim$ & $\begin{array}{l}\text { (aroppea) } \\
.0182706\end{array}$ & .0064432 & 2.84 & 0.005 & .0055176 & .0310235 \\
\hline gdd $\overline{2}$ _verno r & (dropped) & & & & & \\
\hline cpgdd_vern $\sim r$ & (dropped) & & & & & \\
\hline _cons & .7348687 & .0158199 & 46.45 & 0.000 & .7035567 & .7661808 \\
\hline
\end{tabular}




\section{Forks, WA}

Linear regression

$\begin{array}{llr}\text { Number of obs } & & 154 \\ \mathrm{~F}(32,121) & 6.22 \\ \text { Prob }>\text { F } & 0.0000 \\ \text { R-squared } & =0.5993 \\ \text { Root MSE } & = & .05867\end{array}$

\begin{tabular}{|c|c|c|c|c|c|c|}
\hline ndvi_forkswa & Coef. & $\begin{array}{c}\text { Robust } \mathrm{HC} 3 \\
\text { Std. Err. }\end{array}$ & $t$ & $P>|t|$ & [95\% Conf. & Interval] \\
\hline & & & & & & \\
\hline year_1983 & .0060116 & .0267616 & 0.22 & 0.823 & -.04697 & .0589932 \\
\hline year_1984 & .0074518 & .0235332 & 0.32 & 0.752 & -.0391383 & .054042 \\
\hline year_1985 & .0344618 & .0151639 & 2.27 & 0.025 & .0044408 & .0644828 \\
\hline year 1986 & .0149436 & .0181736 & 0.82 & 0.413 & -.0210358 & .0509231 \\
\hline year_1987 & .037665 & .0176966 & 2.13 & 0.035 & .00263 & .0727 \\
\hline year 1988 & .0292729 & .0169949 & 1.72 & 0.088 & -.004373 & .0629188 \\
\hline year_1989 & -.005 & .0133734 & -0.37 & 0.709 & -.0314761 & .0214761 \\
\hline year 1990 & .0124962 & .0234983 & 0.53 & 0.596 & -.0340249 & .0590173 \\
\hline year-1991 & -.0379361 & .0560899 & -0.68 & 0.500 & -.1489808 & .0731086 \\
\hline year 1992 & .0130545 & .0173993 & 0.75 & 0.455 & -.021392 & .047501 \\
\hline year 1993 & .009134 & .0410418 & 0.22 & 0.824 & -.072119 & .090387 \\
\hline year 1994 & .0426475 & .0195099 & 2.19 & 0.031 & .0040226 & .0812725 \\
\hline year_1995 & -.0021399 & .0173761 & -0.12 & 0.902 & -.0365404 & .0322606 \\
\hline year_1996 & -.008113 & .0185604 & -0.44 & 0.663 & -.0448582 & .0286322 \\
\hline year 1997 & -.0364267 & .0424329 & -0.86 & 0.392 & -.1204337 & .0475804 \\
\hline year_1998 & .0318406 & .0178147 & 1.79 & 0.076 & -.0034284 & .0671095 \\
\hline year_1999 & -.1516868 & .0491609 & -3.09 & 0.003 & -.2490138 & -.0543597 \\
\hline year_2000 & .007253 & .0175152 & 0.41 & 0.680 & -.0274229 & .0419289 \\
\hline year_2001 & -.0193826 & .0189798 & -1.02 & 0.309 & -.0569581 & .0181929 \\
\hline year_2002 & -.0509337 & .0402967 & -1.26 & 0.209 & -.1307116 & .0288443 \\
\hline year_2003 & -.0476575 & .0304495 & -1.57 & 0.120 & -.1079404 & .0126253 \\
\hline $\operatorname{may} 1631$ & .0237826 & .0226944 & 1.05 & 0.297 & -.021147 & .0687122 \\
\hline jun115 & .0521723 & .0176589 & 2.95 & 0.004 & .0172119 & .0871326 \\
\hline jun 1630 & .0422529 & .0225308 & 1.88 & 0.063 & -.0023528 & .0868585 \\
\hline jul115 & .1013079 & .0162424 & 6.24 & 0.000 & .0691518 & .1334639 \\
\hline jull631 & .0970547 & .0172559 & 5.62 & 0.000 & .0628921 & .1312172 \\
\hline $\operatorname{aug} 115$ & .0818146 & .0168627 & 4.85 & 0.000 & .0484305 & .1151987 \\
\hline cp_forkswa & -.0060689 & .0119968 & -0.51 & 0.614 & -.0298197 & .0176818 \\
\hline gdd_forkswa & .0037493 & .0019267 & 1.95 & 0.054 & -.0000651 & .0075637 \\
\hline cp2 $2^{-}$forkswa & -.0012039 & .001723 & -0.70 & 0.486 & -.004615 & .0022073 \\
\hline gdd2_forkswa & -.0001173 & .0000558 & -2.10 & 0.038 & -.0002278 & $-6.80 e-06$ \\
\hline cpgdd_ffork a & .000813 & .0006455 & 1.26 & 0.210 & -.0004649 & .0020909 \\
\hline _cons & .7110297 & .0216565 & 32.83 & 0.000 & .6681549 & .7539044 \\
\hline
\end{tabular}


Batesville Livestock, AK

Linear regression

$\begin{array}{llr}\text { Number of obs } & = & 154 \\ \mathrm{~F}(32, \text { 121) } & = & 3.42 \\ \text { Prob }>\text { F } & =0000 \\ \text { R-squared } & =0.3823 \\ \text { Root MSE } & = & .04451\end{array}$

\begin{tabular}{|c|c|c|c|c|c|c|}
\hline ndvi_bates $\sim$ k & Coef. & $\begin{array}{l}\text { Robust } \mathrm{HC} 3 \\
\text { Std. Err. }\end{array}$ & $t$ & $P>|t|$ & {$[95 \%$ Con } & Inte \\
\hline year_1983 & .0114399 & .0214599 & 0.53 & 0.595 & -.0310457 & .0539255 \\
\hline year_1984 & .0240602 & .0257169 & 0.94 & 0.351 & -.0268532 & .0749736 \\
\hline year_1985 & .0273521 & .0195054 & 1.40 & 0.163 & -.011264 & .0659682 \\
\hline year_1986 & -.0294981 & .0294025 & -1.00 & 0.318 & -.0877081 & .028712 \\
\hline year_1987 & -.0139426 & .0285173 & -0.49 & 0.626 & -.0704002 & .042515 \\
\hline year_1988 & .0113771 & .0358711 & 0.32 & 0.752 & -.0596392 & .0823935 \\
\hline year_1989 & .009436 & .0264758 & 0.36 & 0.722 & -.0429797 & .0618518 \\
\hline year_1990 & .0324041 & .0174063 & 1.86 & 0.065 & -.0020562 & .0668644 \\
\hline year_1991 & -.006614 & .0278965 & -0.24 & 0.813 & -.0618424 & .0486145 \\
\hline year-1992 & .0149007 & .0284544 & 0.52 & 0.601 & -.0414322 & .0712336 \\
\hline year_1993 & .0361911 & .0187281 & 1.93 & 0.056 & -.0008861 & .0732683 \\
\hline year_1994 & -.010629 & .0204423 & -0.52 & 0.604 & -.0510999 & .0298418 \\
\hline year_1995 & -.0245433 & .0307518 & -0.80 & 0.426 & -.0854246 & .036338 \\
\hline year_1996 & .0410334 & .0165679 & 2.48 & 0.015 & .0082328 & .073834 \\
\hline year_1997 & .0319769 & .0197825 & 1.62 & 0.109 & -.0071877 & .0711415 \\
\hline year_1998 & .0087645 & .0181049 & 0.48 & 0.629 & -.0270788 & .0446079 \\
\hline year_1999 & -.0552819 & .0251114 & -2.20 & 0.030 & -.1049965 & -.0055674 \\
\hline year_2000 & .0100845 & .0208392 & 0.48 & 0.629 & -.0311722 & .0513412 \\
\hline year_2001 & -.0005663 & .023373 & -0.02 & 0.981 & -.0468394 & .0457068 \\
\hline year_2002 & -.0054917 & .0242415 & -0.23 & 0.821 & -.0534841 & .0425007 \\
\hline year_2003 & .038498 & .0228749 & 1.68 & 0.095 & -.0067889 & .0837849 \\
\hline maȳ1631 & .0319031 & .0182444 & 1.75 & 0.083 & -.0042166 & .0680227 \\
\hline jun115 & .0287722 & .0177867 & 1.62 & 0.108 & -.0064412 & .0639856 \\
\hline jun 1630 & .0168771 & .0200879 & 0.84 & 0.402 & -.0228923 & .0566464 \\
\hline jul115 & -.0036666 & .0235737 & -0.16 & 0.877 & -.0503371 & .0430038 \\
\hline jul1631 & -.0292008 & .0265974 & -1.10 & 0.274 & -.0818573 & .0234557 \\
\hline $\operatorname{aug} 115$ & -.0275749 & .0203801 & -1.35 & 0.179 & -.0679227 & .0127729 \\
\hline tesvi k & -.0129744 & .0182871 & -0.71 & 0.479 & -.0491784 & .0232297 \\
\hline gdd_batesv $\sim \mathrm{k}$ & .000138 & .0004328 & 0.32 & 0.750 & -.0007188 & .0009948 \\
\hline cp2_batesv $\sim k$ & -.0000354 & .0030598 & -0.01 & 0.991 & -.0060932 & .0060224 \\
\hline gddי2_bates $\sim \mathrm{k}$ & $-5.75 e-07$ & $1.60 e-06$ & -0.36 & 0.720 & $-3.74 e-06$ & $2.59 e-06$ \\
\hline $\bar{d}$ bate $\sim k$ & .0001726 & .0001086 & 1.59 & 0.115 & -.0000425 & .0003876 \\
\hline cons & .7953897 & .0289329 & 27.49 & 0.000 & .7381093 & .85267 \\
\hline
\end{tabular}




\section{Harrington, WA}

Linear regression

$\begin{array}{llr}\text { Number of obs } & = & 154 \\ \mathrm{~F}(32, \text { 121) } & 13.43 \\ \text { Prob }>\text { F } & 0.0000 \\ \text { R-squared } & =0.6796 \\ \text { Root MSE } & = & .06054\end{array}$

\begin{tabular}{|c|c|c|c|c|c|c|}
\hline ndvi_harri a & Coef. & $\begin{array}{l}\text { Robust } \mathrm{HC} 3 \\
\text { Std. Err. }\end{array}$ & $t$ & $P>|t|$ & [95\% Con & val] \\
\hline year_1983 & .0055411 & .0205157 & 0.27 & 0.788 & -.035075 & .0461573 \\
\hline year 1984 & .0766641 & .0454047 & 1.69 & 0.094 & -.0132265 & .1665548 \\
\hline year 1985 & -.0120521 & .0331741 & -0.36 & 0.717 & -.0777289 & .0536247 \\
\hline year 1986 & -.0116291 & .0329141 & -0.35 & 0.724 & -.0767912 & .053533 \\
\hline year-1987 & -.0305642 & .0370841 & -0.82 & 0.411 & -.103982 & .0428535 \\
\hline year 1988 & .0077081 & .0252721 & 0.31 & 0.761 & -.0423247 & .0577408 \\
\hline year 1989 & -.0439594 & .0255772 & -1.72 & 0.088 & -.0945962 & .0066774 \\
\hline year_1990 & -.0102323 & .0369698 & -0.28 & 0.782 & -.0834237 & .0629591 \\
\hline year_1991 & -.0373052 & .0403143 & -0.93 & 0.357 & -.1171181 & .0425077 \\
\hline year 1992 & -.0272441 & .0286327 & -0.95 & 0.343 & -.0839301 & .0294419 \\
\hline year 1993 & .0314648 & .0251197 & 1.25 & 0.213 & -.0182662 & .0811958 \\
\hline year_1994 & .0320917 & .0404669 & 0.79 & 0.429 & -.0480231 & .1122066 \\
\hline year_1995 & .0129281 & .0232946 & 0.55 & 0.580 & -.0331896 & .0590458 \\
\hline year_1996 & .073149 & .0236012 & 3.10 & 0.002 & .0264241 & .1198738 \\
\hline year_1997 & .0460551 & .0359358 & 1.28 & 0.202 & -.0250892 & .1171995 \\
\hline year_1998 & .0049448 & .0252352 & 0.20 & 0.845 & -.0450148 & .0549045 \\
\hline year_1999 & -.0233893 & .0450894 & -0.52 & 0.605 & -.1126556 & .065877 \\
\hline year_2000 & .0550375 & .0307794 & 1.79 & 0.076 & -.0058984 & .1159734 \\
\hline year 2001 & .000926 & .0244811 & 0.04 & 0.970 & -.0475407 & .0493927 \\
\hline year 2002 & .0396546 & .0275035 & 1.44 & 0.152 & -.0147958 & .0941051 \\
\hline year 2003 & .0168712 & .0225081 & 0.75 & 0.455 & -.0276895 & .061432 \\
\hline $\operatorname{may} 1631$ & .036015 & .0230923 & 1.56 & 0.121 & -.0097023 & .0817322 \\
\hline jun115 & .0631406 & .0207211 & 3.05 & 0.003 & .0221177 & .1041634 \\
\hline jun 1630 & .041912 & .0193342 & 2.17 & 0.032 & .0036348 & .0801892 \\
\hline jul115 & -.0162657 & .023047 & -0.71 & 0.482 & -.0618933 & .0293619 \\
\hline jul1631 & -.0901115 & .030048 & -3.00 & 0.003 & -.1495995 & -.0306235 \\
\hline $\operatorname{aug} 115$ & -.1629119 & .0198165 & -8.22 & 0.000 & -.2021439 & -.12368 \\
\hline arring a & -.0576166 & .0544911 & -1.06 & 0.292 & -.1654962 & .050263 \\
\hline gdd_harrin a & .0003631 & .0007002 & 0.52 & 0.605 & -.0010232 & .0017494 \\
\hline cp2 harrin a & .0192197 & .0335473 & 0.57 & 0.568 & -.0471961 & .0856354 \\
\hline gdd $\overline{2}$ harri a & $-6.30 e-06$ & $4.85 e-06$ & -1.30 & 0.197 & -.0000159 & $3.31 e-06$ \\
\hline cpgdd̄_harr a & .0006729 & .0012371 & 0.54 & 0.587 & -.0017762 & .003122 \\
\hline _cons & .4382462 & .0247085 & 17.74 & 0.000 & .3893293 & .4871632 \\
\hline
\end{tabular}




\section{Everett, WA}

Linear regression

$\begin{array}{llr}\text { Number of obs } & = & 154 \\ \mathrm{~F}(29, \quad 124) & = & 3.81 \\ \text { Prob }>\text { F } & = & 0.0000 \\ \text { R-squared } & = & 0.3803 \\ \text { Root MSE } & = & .06163\end{array}$

\begin{tabular}{|c|c|c|c|c|c|c|}
\hline ndvi_evere a & Coef. & $\begin{array}{l}\text { Robust } \mathrm{HC} 3 \\
\text { Std. Err. }\end{array}$ & $t$ & $P>|t|$ & {$\left[\begin{array}{l}95 \% \\
\text { Conf. }\end{array}\right.$} & Interval] \\
\hline year_1983 & .0078865 & .0363492 & 0.22 & 0.829 & -.0640588 & .0798318 \\
\hline year_1984 & -.1002617 & .0452808 & -2.21 & 0.029 & -.1898851 & -.0106384 \\
\hline year_1985 & .0401845 & .0532152 & 0.76 & 0.452 & -.0651434 & .1455123 \\
\hline year_1986 & -.0418361 & .0578241 & -0.72 & 0.471 & -.1562863 & .072614 \\
\hline year_1987 & -.0008327 & .0462247 & -0.02 & 0.986 & -.0923244 & .0906589 \\
\hline year_1988 & .0312552 & .0376105 & 0.83 & 0.408 & -.0431865 & .1056968 \\
\hline year_1989 & -.0327985 & .0305674 & -1.07 & 0.285 & -.0932999 & .0277029 \\
\hline year_1990 & .0293598 & .037918 & 0.77 & 0.440 & -.0456907 & .1044102 \\
\hline year_1991 & .0345358 & .0307125 & 1.12 & 0.263 & -.0262528 & .0953245 \\
\hline year_1992 & .0545449 & .0301925 & 1.81 & 0.073 & -.0052145 & .1143042 \\
\hline year_1993 & .0256055 & .031942 & 0.80 & 0.424 & -.0376167 & .0888277 \\
\hline year_1994 & .031292 & .0331575 & 0.94 & 0.347 & -.0343359 & .09692 \\
\hline year_1995 & .0437613 & .0331105 & 1.32 & 0.189 & -.0217737 & .1092963 \\
\hline year_1996 & .0172396 & .037226 & 0.46 & 0.644 & -.0564411 & .0909203 \\
\hline year_1997 & .0512731 & .0337761 & 1.52 & 0.132 & -.0155792 & .1181254 \\
\hline year_1998 & .0080893 & .0393928 & 0.21 & 0.838 & -.06988 & .0860587 \\
\hline year_1999 & -.0334409 & .0442952 & -0.75 & 0.452 & -.1211136 & .0542318 \\
\hline year_2000 & -.025353 & .0424398 & -0.60 & 0.551 & -.1093532 & .0586472 \\
\hline year_2001 & .0176677 & .0303028 & 0.58 & 0.561 & -.0423101 & .0776455 \\
\hline year_2002 & -.0044576 & .0303636 & -0.15 & 0.884 & -.0645556 & .0556403 \\
\hline year_2003 & -.0133534 & .0308478 & -0.43 & 0.666 & -.0744098 & .047703 \\
\hline may1631 & .0092866 & .0177648 & 0.52 & 0.602 & -.025875 & .0444482 \\
\hline jun115 & .0184379 & .0233206 & 0.79 & 0.431 & -.02772 & .0645958 \\
\hline jun1630 & .0113146 & .0206087 & 0.55 & 0.584 & -.0294758 & .052105 \\
\hline jul115 & .0363078 & .0169709 & 2.14 & 0.034 & .0027177 & .069898 \\
\hline jul1631 & .0484227 & .0184804 & 2.62 & 0.010 & .0118449 & .0850006 \\
\hline aug115 & .0394944 & .0169896 & 2.32 & 0.022 & .0058673 & .0731216 \\
\hline verettwa & -.0235369 & .0239141 & -0.98 & 0.327 & -.0708695 & .0237957 \\
\hline veret a & (dropped) & & & & & \\
\hline cp2 everet a & .0043043 & .008742 & 0.49 & 0.623 & -.0129986 & .0216071 \\
\hline $\begin{array}{l}\text { gdd } 2 \text { evere a } \\
\text { cpgdd ever a }\end{array}$ & $\begin{array}{l}\text { (dropped) } \\
\text { (dropped) }\end{array}$ & & & & & \\
\hline _cons & .4910288 & .035969 & 13.65 & 0.000 & .4198361 & .5622216 \\
\hline
\end{tabular}


Eltopia, WA

Linear regression

$\begin{array}{llr}\text { Number of obs } & & 154 \\ \mathrm{~F}(32, \text { 121) } & & 6.50 \\ \text { Prob }>\text { F } & =.0000 \\ \text { R-squared } & = & 0.5554 \\ \text { Root MSE } & = & .0381\end{array}$

\begin{tabular}{|c|c|c|c|c|c|c|}
\hline ndvi_eltop a & Coef. & $\begin{array}{l}\text { Robust } \mathrm{HC} 3 \\
\text { Std. Err. }\end{array}$ & $t$ & $P>|t|$ & {$[95 \% \mathrm{Co}$} & Int \\
\hline year_1983 & -.0054505 & .0228514 & -0.24 & 0.812 & -.0506909 & .0397899 \\
\hline year_1984 & .0264788 & .0304288 & 0.87 & 0.386 & -.0337631 & .0867207 \\
\hline year_1985 & -.0160062 & .0184062 & -0.87 & 0.386 & -.0524461 & .0204337 \\
\hline year 1986 & .008303 & .0179716 & 0.46 & 0.645 & -.0272764 & .0438824 \\
\hline year_1987 & -.0170675 & .0184416 & -0.93 & 0.357 & -.0535776 & .0194426 \\
\hline year_1988 & -.0198588 & .0190906 & -1.04 & 0.300 & -.0576538 & .0179361 \\
\hline year_1989 & -.052756 & .0246561 & -2.14 & 0.034 & -.1015693 & -.0039427 \\
\hline year_1990 & -.0044378 & .0192835 & -0.23 & 0.818 & -.0426145 & .0337389 \\
\hline year_1991 & .0036248 & .0215183 & 0.17 & 0.867 & -.0389763 & .046226 \\
\hline year_1992 & .0227361 & .0214062 & 1.06 & 0.290 & -.019643 & .0651153 \\
\hline year_1993 & -.0089431 & .0230479 & -0.39 & 0.699 & -.0545726 & .0366863 \\
\hline year_1994 & .0349018 & .0188267 & 1.85 & 0.066 & -.0023705 & .0721742 \\
\hline year_1995 & .0335978 & .0211478 & 1.59 & 0.115 & -.0082699 & .0754655 \\
\hline year_1996 & .0410858 & .0217296 & 1.89 & 0.061 & -.0019337 & .0841053 \\
\hline year_1997 & .0148293 & .0231096 & 0.64 & 0.522 & -.0309224 & .0605809 \\
\hline year_1998 & .0397488 & .0184079 & 2.16 & 0.033 & .0033055 & .0761921 \\
\hline year_1999 & -.0973181 & .0381466 & -2.55 & 0.012 & -.1728393 & -.0217968 \\
\hline year_2000 & .0698279 & .0226531 & 3.08 & 0.003 & .0249801 & .1146756 \\
\hline year_2001 & .0042403 & .019066 & 0.22 & 0.824 & -.0335059 & .0419866 \\
\hline year_2002 & -.0058253 & .0239614 & -0.24 & 0.808 & -.0532633 & .0416126 \\
\hline year_2003 & .0161297 & .0257304 & 0.63 & 0.532 & -.0348105 & .0670699 \\
\hline maȳ1631 & .0180481 & .0119617 & 1.51 & 0.134 & -.0056333 & .0417294 \\
\hline jun115 & .03723 & .0111594 & 3.34 & 0.001 & .015137 & .0593229 \\
\hline jun1630 & .0480014 & .0122283 & 3.93 & 0.000 & .0237923 & .0722104 \\
\hline jul115 & .0660735 & .0125847 & 5.25 & 0.000 & .0411587 & .0909883 \\
\hline jul1631 & .0668289 & .0117565 & 5.68 & 0.000 & .0435539 & .0901039 \\
\hline $\operatorname{aug} 115$ & .0411103 & .0140927 & 2.92 & 0.004 & .0132101 & .0690105 \\
\hline eltopiawa & -.0025721 & .0344842 & -0.07 & 0.941 & -.0708426 & .0656985 \\
\hline gdd_eltopi a & -.0007395 & .0004285 & -1.73 & 0.087 & -.0015878 & .0001089 \\
\hline cp2_eltopi a & -.0124231 & .0292005 & -0.43 & 0.671 & -.0702333 & .045387 \\
\hline gdd2_eltop a & $5.55 e-06$ & $4.65 e-06$ & 1.19 & 0.234 & $-3.65 e-06$ & .0000147 \\
\hline cpgdd_elto a & .0001789 & .0004249 & 0.42 & 0.674 & -.0006623 & .00102 \\
\hline cons & .6073597 & .0177403 & 34.24 & 0.000 & .5722382 & .6424812 \\
\hline
\end{tabular}




\section{Brandenberg, MT}

Linear regression

$\begin{array}{llr}\text { Number of obs } & = & 154 \\ \mathrm{~F}(32, \text { 121) } & = & 7.94 \\ \text { Prob }>\text { F } & =00000 \\ \text { R-squared } & =0.6524 \\ \text { Root MSE } & = & .04388\end{array}$

\begin{tabular}{|c|c|c|c|c|c|c|}
\hline ndvi_brand $t$ & Coef. & $\begin{array}{l}\text { Robust } \mathrm{HC} 3 \\
\text { Std. Err. }\end{array}$ & $t$ & $P>|t|$ & [95\% Con & Inter \\
\hline year_1983 & -.035058 & .0204923 & -1.71 & 0.090 & -.0756279 & .005512 \\
\hline year_1984 & -.0101507 & .0206373 & -0.49 & 0.624 & -.0510076 & .0307063 \\
\hline year_1985 & -.089907 & .0216681 & -4.15 & 0.000 & -.1328047 & -.0470093 \\
\hline year_1986 & -.0257952 & .0345847 & -0.75 & 0.457 & -.0942647 & .0426744 \\
\hline year_1987 & -.0421632 & .0215427 & -1.96 & 0.053 & -.0848127 & .0004863 \\
\hline year_1988 & -.0828801 & .0263894 & -3.14 & 0.002 & -.1351248 & -.0306354 \\
\hline year_1989 & -.0318315 & .0200625 & -1.59 & 0.115 & -.0715506 & .0078876 \\
\hline year_1990 & .0380422 & .0285558 & 1.33 & 0.185 & -.0184915 & .094576 \\
\hline year_1991 & .0673894 & .0287816 & 2.34 & 0.021 & .0104086 & .1243701 \\
\hline year-1992 & -.0162726 & .0216585 & -0.75 & 0.454 & -.0591514 & .0266062 \\
\hline year_1993 & .0196176 & .0355546 & 0.55 & 0.582 & -.0507721 & .0900073 \\
\hline year_1994 & .0357144 & .0264396 & 1.35 & 0.179 & -.0166299 & .0880587 \\
\hline year_1995 & .0573181 & .0205729 & 2.79 & 0.006 & .0165886 & .0980475 \\
\hline year_1996 & -.0019184 & .0289754 & -0.07 & 0.947 & -.0592828 & .055446 \\
\hline year_1997 & .0369192 & .0191129 & 1.93 & 0.056 & -.0009199 & .0747583 \\
\hline year_1998 & -.075126 & .0230106 & -3.26 & 0.001 & -.1206815 & -.0295704 \\
\hline year_1999 & -.0154962 & .0263867 & -0.59 & 0.558 & -.0677356 & .0367433 \\
\hline year_2000 & -.0203191 & .0225604 & -0.90 & 0.370 & -.0649835 & .0243452 \\
\hline year_2001 & -.0209183 & .0205062 & -1.02 & 0.310 & -.0615157 & .019679 \\
\hline year_2002 & -.0717929 & .0191303 & -3.75 & 0.000 & -.1096664 & -.0339194 \\
\hline year_2003 & .0301925 & .0184446 & 1.64 & 0.104 & -.0063234 & .0667084 \\
\hline maȳ1631 & .0531057 & .0173314 & 3.06 & 0.003 & .0187937 & .0874177 \\
\hline jun115 & .0783822 & .016955 & 4.62 & 0.000 & .0448153 & .111949 \\
\hline jun 1630 & .0894496 & .0195981 & 4.56 & 0.000 & .0506499 & .1282493 \\
\hline jul115 & .0724566 & .0206259 & 3.51 & 0.001 & .0316221 & .113291 \\
\hline jul1631 & .0490062 & .0214487 & 2.28 & 0.024 & .0065429 & .0914694 \\
\hline $\operatorname{aug} 115$ & .0228609 & .0231244 & 0.99 & 0.325 & -.02292 & .0686417 \\
\hline anden $\sim t$ & -.0101959 & .0289472 & -0.35 & 0.725 & -.0675045 & .0471127 \\
\hline gdd_brande $\sim t$ & -.0005747 & .0008883 & -0.65 & 0.519 & -.0023332 & .0011839 \\
\hline cp2_brande $\sim t$ & .0003473 & .0084431 & 0.04 & 0.967 & -.0163681 & .0170627 \\
\hline dd 2 _brand $\sim t$ & $1.77 e-06$ & $6.27 e-06$ & 0.28 & 0.778 & -.0000106 & .0000142 \\
\hline de_bran t & .0002667 & .0005082 & 0.52 & 0.601 & -.0007395 & .0012729 \\
\hline _cons & .3949821 & .0222613 & 17.74 & 0.000 & .35091 & .4390542 \\
\hline
\end{tabular}


Flatwillow, MT

Linear regression

$\begin{array}{llr}\text { Number of obs } & & 154 \\ \text { F }(32,121) & 4.87 \\ \text { Prob }>\text { F } & =0000 \\ \text { R-squared } & =0.5757 \\ \text { Root MSE } & = & .06548\end{array}$

\begin{tabular}{|c|c|c|c|c|c|c|}
\hline ndvi_flatw t & Coef. & $\begin{array}{l}\text { Robust HC3 } \\
\text { Std. Err. }\end{array}$ & $t$ & $P>|t|$ & {$[95 \%$ Con $]$} & Interval] \\
\hline year_1983 & -.0711901 & .0342244 & -2.08 & 0.040 & -.1389464 & -.0034339 \\
\hline year_1984 & -.0353796 & .0346689 & -1.02 & 0.310 & -.1040158 & .0332566 \\
\hline year_1985 & -.1371474 & .0349318 & -3.93 & 0.000 & -.2063041 & -.0679907 \\
\hline year_1986 & -.0044888 & .0491437 & -0.09 & 0.927 & -.1017818 & .0928041 \\
\hline year_1987 & -.0549796 & .0372079 & -1.48 & 0.142 & -.1286425 & .0186833 \\
\hline year_1988 & -.0322294 & .0374669 & -0.86 & 0.391 & -.1064049 & .0419461 \\
\hline year_1989 & .0293306 & .0399017 & 0.74 & 0.464 & -.0496654 & .1083267 \\
\hline year_1990 & -.0255475 & .031799 & -0.80 & 0.423 & -.088502 & .0374069 \\
\hline year_1991 & .0910567 & .0518738 & 1.76 & 0.082 & -.0116412 & .1937545 \\
\hline year_1992 & -.1042937 & .0570518 & -1.83 & 0.070 & -.2172428 & .0086554 \\
\hline year 1993 & .0456646 & .0363511 & 1.26 & 0.211 & -.0263021 & .1176313 \\
\hline year_1994 & .0622018 & .0332574 & 1.87 & 0.064 & -.00364 & .1280436 \\
\hline year_1995 & .0386843 & .0406378 & 0.95 & 0.343 & -.041769 & .1191375 \\
\hline year_1996 & .0099276 & .0315225 & 0.31 & 0.753 & -.0524796 & .0723347 \\
\hline year_1997 & -.0858331 & .0359387 & -2.39 & 0.018 & -.1569833 & -.014683 \\
\hline year_1998 & -.0021259 & .0402146 & -0.05 & 0.958 & -.0817414 & .0774896 \\
\hline year_1999 & -.0361191 & .0313523 & -1.15 & 0.252 & -.0981892 & .0259509 \\
\hline year_2000 & -.1008027 & .0336714 & -2.99 & 0.003 & -.1674641 & -.0341414 \\
\hline year_2001 & -.0402148 & .0413954 & -0.97 & 0.333 & -.122168 & .0417384 \\
\hline year_2002 & -.0627272 & .0357959 & -1.75 & 0.082 & -.1335947 & .0081403 \\
\hline year_2003 & -.0124968 & .036162 & -0.35 & 0.730 & -.084089 & .0590955 \\
\hline $\operatorname{may} 1631$ & .0244526 & .0192479 & 1.27 & 0.206 & -.0136536 & .0625589 \\
\hline jun115 & .0449678 & .0202331 & 2.22 & 0.028 & .0049111 & .0850246 \\
\hline jun 1630 & .0802566 & .0217397 & 3.69 & 0.000 & .0372173 & .123296 \\
\hline jul115 & .0739652 & .0249214 & 2.97 & 0.004 & .0246268 & .1233037 \\
\hline jul1631 & .0336613 & .0313226 & 1.07 & 0.285 & -.0283501 & .0956726 \\
\hline $\operatorname{aug} 115$ & .0232828 & .0330507 & 0.70 & 0.483 & -.0421498 & .0887154 \\
\hline flatwil $\sim t$ & .017896 & .0223153 & 0.80 & 0.424 & -.0262831 & .0620751 \\
\hline gdd_flatwi t & -.0004215 & .0006758 & -0.62 & 0.534 & -.0017595 & .0009165 \\
\hline cp2_flatwi t & -.0062353 & .0055486 & -1.12 & 0.263 & -.0172202 & .0047496 \\
\hline gdd2_flatw t & $-8.35 e-07$ & $3.11 e-06$ & -0.27 & 0.789 & $-6.99 e-06$ & $5.32 e-06$ \\
\hline 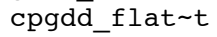 & -.0000981 & .0003654 & -0.27 & 0.789 & -.0008215 & .0006252 \\
\hline _cons & .3182767 & .0331264 & 9.61 & 0.000 & .2526942 & .3838592 \\
\hline
\end{tabular}




\section{Brownfield, TX}

Linear regression

Number of obs $=$

$F(30,123)=$

Prob $>\mathrm{F}=0.0000$

R-squared $=1.0000$

Root MSE $=1.4 \mathrm{e}-0$

\begin{tabular}{|c|c|c|c|c|c|c|}
\hline & & Robust $\mathrm{HC} 3$ & & & & \\
\hline ndvi_brown $\sim \mathrm{x}$ & Coef. & Std. Err. & $t$ & $P>|t|$ & [95\% Conf. & Interval] \\
\hline year 1983 & $9.11 e-09$ & $4.67 e-09$ & 1.95 & 0.053 & $-1.23 e-10$ & $1.83 e-08$ \\
\hline year_1984 & $1.10 \mathrm{e}-09$ & $5.34 e-09$ & 0.21 & 0.838 & $-9.48 e-09$ & $1.17 e-08$ \\
\hline year_1985 & $-5.35 e-09$ & $3.75 e-09$ & -1.43 & 0.156 & $-1.28 e-08$ & $2.08 e-09$ \\
\hline year_1986 & $-4.07 e-09$ & $5.12 e-09$ & -0.79 & 0.429 & $-1.42 e-08$ & $6.07 e-09$ \\
\hline year_1987 & $7.68 e-11$ & $5.37 e-09$ & 0.01 & 0.989 & $-1.06 e-08$ & $1.07 e-08$ \\
\hline year_1988 & $-3.46 e-09$ & $6.18 e-09$ & -0.56 & 0.577 & $-1.57 e-08$ & $8.78 e-09$ \\
\hline year_1989 & $1.82 e-09$ & $6.00 e-09$ & 0.30 & 0.763 & $-1.01 e-08$ & $1.37 e-08$ \\
\hline year_1990 & $-2.82 e-09$ & $4.24 e-09$ & -0.67 & 0.507 & $-1.12 e-08$ & $5.57 e-09$ \\
\hline year_1991 & $-8.66 e-10$ & $9.13 e-09$ & -0.09 & 0.925 & $-1.89 e-08$ & $1.72 e-08$ \\
\hline year_1992 & $-3.09 e-09$ & $7.20 e-09$ & -0.43 & 0.668 & $-1.73 e-08$ & $1.12 e-08$ \\
\hline year_1993 & $7.44 e-09$ & $5.70 e-09$ & 1.31 & 0.194 & $-3.84 e-09$ & $1.87 e-08$ \\
\hline year-1994 & $-5.89 e-09$ & $5.25 e-09$ & -1.12 & 0.264 & $-1.63 e-08$ & $4.49 e-09$ \\
\hline year_1995 & $8.65 e-09$ & $5.62 e-09$ & 1.54 & 0.127 & $-2.48 e-09$ & $1.98 e-08$ \\
\hline year_1996 & $5.92 e-09$ & $1.36 e-08$ & 0.44 & 0.664 & $-2.09 e-08$ & $3.28 e-08$ \\
\hline year_1997 & $4.54 e-10$ & $5.63 e-09$ & 0.08 & 0.936 & $-1.07 e-08$ & $1.16 \mathrm{e}-08$ \\
\hline year_1998 & $3.29 e-09$ & $5.65 e-09$ & 0.58 & 0.561 & $-7.89 e-09$ & $1.45 e-08$ \\
\hline year_1999 & $-3.74 e-09$ & $6.37 e-09$ & -0.59 & 0.558 & $-1.63 e-08$ & $8.86 e-09$ \\
\hline year_2000 & $7.85 e-09$ & $7.85 e-09$ & 1.00 & 0.319 & $-7.69 e-09$ & $2.34 e-08$ \\
\hline year_2001 & $3.57 e-09$ & 6.53 & 0.55 & 0.586 & $-9.36 e-09$ & $1.65 e-08$ \\
\hline year_2002 & $-7.57 e-09$ & $1.09 e-08$ & -0.69 & 0.489 & $-2.92 e-08$ & $1.40 e-08$ \\
\hline year_2003 & $-2.47 e-09$ & $4.91 e-09$ & -0.50 & 0.615 & $-1.22 e-08$ & $7.24 e-09$ \\
\hline $\operatorname{maȳ} 1631$ & $3.16 e-10$ & $3.17 e-09$ & 0.10 & 0.921 & $-5.97 e-09$ & $6.60 e-09$ \\
\hline jun115 & $-4.53 e-09$ & $3.97 e-09$ & -1.14 & 0.257 & $-1.24 e-08$ & $3.34 e-09$ \\
\hline jun1630 & $-3.25 e-09$ & $3.22 \mathrm{e}-09$ & -1.01 & 0.315 & $-9.63 e-09$ & $3.13 e-09$ \\
\hline jul115 & $-5.53 e-10$ & $4.05 e-09$ & -0.14 & 0.891 & $-8.56 e-09$ & $7.45 e-09$ \\
\hline jul1631 & $1.40 e-09$ & $5.04 e-09$ & 0.28 & 0.782 & $-8.59 e-09$ & $1.14 \mathrm{e}-08$ \\
\hline $\operatorname{aug} 115$ & $-6.27 e-09$ & $8.24 \mathrm{e}-09$ & -0.76 & 0.448 & $-2.26 e-08$ & $1.00 \mathrm{e}-08$ \\
\hline $\begin{array}{l}\text { cp brownfi x } \\
\text { gdd brownf } \sim x\end{array}$ & $\begin{array}{r}-1.63 e-10 \\
\text { (dropped) }\end{array}$ & $1.04 \mathrm{e}-09$ & -0.16 & 0.876 & $-2.22 e-09$ & $1.90 e-09$ \\
\hline $\begin{array}{l}\text { cp } 2 \text { brown } \sim x \\
\text { gdd2 brown } \sim x\end{array}$ & $4.82 \mathrm{e}-07$ & $6.16 e-07$ & 0.78 & 0.435 & $-7.37 e-07$ & $1.70 e-06$ \\
\hline cpgdd_brow $\sim \mathrm{x}$ & 99999.97 & .03 & & 0.000 & 99999.9 & 100000 \\
\hline cons & $4.14 \mathrm{e}-08$ & $5.28 e-08$ & 0.78 & 0.434 & $-6.31 e-08$ & $1.46 \mathrm{e}-07$ \\
\hline
\end{tabular}




\title{
Gonzales, TX
}

\author{
Linear regression
}

$\begin{array}{llr}\text { Number of obs } & = & 153 \\ \text { F }(32, \quad 120) & 10.67 \\ \text { Prob F } & = & 0.0000 \\ \text { R-squared } & =0.6141 \\ \text { Root MSE } & = & .05492\end{array}$

\begin{tabular}{|c|c|c|c|c|c|c|}
\hline ndvi_gonza x & Coef. & $\begin{array}{l}\text { Robust HC3 } \\
\text { Std. Err. }\end{array}$ & $t$ & $P>|t|$ & [ $95 \%$ Conf. & Inte \\
\hline year_1983 & -.0144496 & .0265544 & -0.54 & 0.587 & -.0670254 & .0381262 \\
\hline year_1984 & -.0799375 & .0227925 & -3.51 & 0.001 & -.1250651 & -.0348099 \\
\hline year_1985 & -.0247815 & .0330578 & -0.75 & 0.455 & -.0902336 & .0406706 \\
\hline year_1986 & -.0753549 & .0304998 & -2.47 & 0.015 & -.1357424 & -.0149674 \\
\hline year_1987 & -.0117619 & .0423186 & -0.28 & 0.782 & -.0955499 & .072026 \\
\hline year_1988 & -.0123276 & .0210351 & -0.59 & 0.559 & -.0539755 & .0293204 \\
\hline year_1989 & .0030493 & .0268298 & 0.11 & 0.910 & -.0500718 & .0561704 \\
\hline year_1990 & -.0354235 & .0268341 & -1.32 & 0.189 & -.0885532 & .0177061 \\
\hline year_1991 & .0171802 & .019386 & 0.89 & 0.377 & -.0212028 & .0555633 \\
\hline year_1992 & .0215996 & .0238013 & 0.91 & 0.366 & -.0255254 & .0687245 \\
\hline year_1993 & .0263485 & .0184931 & 1.42 & 0.157 & -.0102665 & .0629635 \\
\hline year_1994 & -.0246681 & .0332899 & -0.74 & 0.460 & -.0905798 & .0412436 \\
\hline year_1995 & -.0084938 & .0229482 & -0.37 & 0.712 & -.0539296 & .036942 \\
\hline year_1996 & -.2032947 & .0219959 & -9.24 & 0.000 & -.2468451 & -.1597444 \\
\hline year_1997 & .0246708 & .0301989 & 0.82 & 0.416 & -.0351209 & .0844625 \\
\hline year_1998 & -.0703109 & .0313554 & -2.24 & 0.027 & -.1323923 & -.0082294 \\
\hline year_1999 & -.036101 & .0577306 & -0.63 & 0.533 & -.1504036 & .0782017 \\
\hline year_2000 & -.0171373 & .0270372 & -0.63 & 0.527 & -.0706691 & .0363944 \\
\hline year_2001 & -.1003226 & .0247054 & -4.06 & 0.000 & -.1492376 & -.0514076 \\
\hline year_2002 & -.0557386 & .0376863 & -1.48 & 0.142 & -.1303549 & .0188776 \\
\hline year_2003 & -.0387039 & .04726 & -0.82 & 0.414 & -.1322755 & .0548677 \\
\hline maȳ1631 & -.0079181 & .0173988 & -0.46 & 0.650 & -.0423664 & .0265302 \\
\hline jun115 & -.0106623 & .0153801 & -0.69 & 0.489 & -.0411138 & .0197892 \\
\hline jun1630 & -.0458304 & .0190463 & -2.41 & 0.018 & -.0835409 & -.00812 \\
\hline jul115 & -.0589957 & .0182459 & -3.23 & 0.002 & -.0951213 & -.0228702 \\
\hline jul1631 & -.0610699 & .0188058 & -3.25 & 0.002 & -.0983041 & -.0238357 \\
\hline $\operatorname{aug} 115$ & -.1089208 & .0204166 & -5.33 & 0.000 & -.1493442 & -.0684974 \\
\hline onzale x & -.000323 & .0094962 & -0.03 & 0.973 & -.0191248 & .0184788 \\
\hline gdd__gonzal x & -.0014917 & .0220651 & -0.07 & 0.946 & -.0451791 & .0421956 \\
\hline cp2_gonzal x & -.0003094 & .0016086 & -0.19 & 0.848 & -.0034943 & .0028755 \\
\hline gdd $\overline{2}$ _gonza x & -.000053 & .0015835 & -0.03 & 0.973 & -.0031882 & .0030821 \\
\hline cpgdd__gonz $\mathrm{x}$ & .0015672 & .0057913 & 0.27 & 0.787 & -.0098992 & .0130336 \\
\hline cons & .6428716 & .022102 & 29.09 & 0.000 & .5991112 & .6866319 \\
\hline
\end{tabular}




\section{Matagorda, TX}

Linear regression

$\begin{array}{llr}\text { Number of obs } & & 153 \\ \text { F }(32, \quad 120) & = & 3.08 \\ \text { Prob F } & = & 0.0000 \\ \text { R-squared } & =0.4643 \\ \text { Root MSE } & = & .04852\end{array}$

\begin{tabular}{|c|c|c|c|c|c|c|}
\hline dvi_matag x & Coef. & $\begin{array}{l}\text { Robust } \mathrm{HC} 3 \\
\text { Std. Err. }\end{array}$ & $t$ & $P>|t|$ & {$[95 \%$ Con } & Int \\
\hline year 1983 & -.0782358 & .0350826 & -2.23 & 0.028 & -.147697 & -.0087746 \\
\hline year_1984 & .0171671 & .0185538 & 0.93 & 0.357 & -.0195682 & .0539024 \\
\hline year 1985 & .0381115 & .0171514 & 2.22 & 0.028 & .0041529 & .0720701 \\
\hline year_1986 & .0143441 & .0171438 & 0.84 & 0.404 & -.0195994 & .0482877 \\
\hline year_1987 & .0151823 & .0190196 & 0.80 & 0.426 & -.0224751 & .0528397 \\
\hline year_1988 & -.0212414 & .0178567 & -1.19 & 0.237 & -.0565966 & .0141137 \\
\hline year_1989 & .0591108 & .0298439 & 1.98 & 0.050 & .000022 & .1181997 \\
\hline year_1990 & -.0181945 & .0231746 & -0.79 & 0.434 & -.0640785 & .0276896 \\
\hline year_1991 & -.0198599 & .0302423 & -0.66 & 0.513 & -.0797375 & .0400177 \\
\hline year_1992 & .0494719 & .0245251 & 2.02 & 0.046 & .0009139 & .09803 \\
\hline year_1993 & .0327257 & .0195735 & 1.67 & 0.097 & -.0060285 & .0714798 \\
\hline year_1994 & .0330454 & .0230282 & 1.43 & 0.154 & -.0125489 & .0786397 \\
\hline year 1995 & .0330833 & .0305168 & 1.08 & 0.280 & -.0273378 & .0935045 \\
\hline year 1996 & -.0472487 & .0209275 & -2.26 & 0.026 & -.0886837 & -.0058137 \\
\hline year_1997 & -.0414385 & .0275005 & -1.51 & 0.134 & -.0958876 & .0130107 \\
\hline year_1998 & -.0842867 & .0317224 & -2.66 & 0.009 & -.1470949 & -.0214785 \\
\hline year 1999 & -.0776512 & .0570838 & -1.36 & 0.176 & -.1906733 & .0353708 \\
\hline year 2000 & -.0557878 & .0342772 & -1.63 & 0.106 & -.1236544 & .0120787 \\
\hline year_2001 & -.0771688 & .032441 & -2.38 & 0.019 & -.1413997 & -.0129378 \\
\hline year_2002 & -.0874113 & 104 & -2.35 & 0.020 & -.1610852 & -.0137374 \\
\hline year_2003 & -.0460176 & .0353892 & -1.30 & 0.196 & -.1160858 & .0240505 \\
\hline maȳ1631 & .0184425 & .0198141 & 0.93 & 0.354 & -.0207881 & .0576732 \\
\hline jun115 & .0268049 & .0260737 & 1.03 & 0.306 & -.0248192 & .0784289 \\
\hline jun 1630 & .0416149 & .0337393 & 1.23 & 0.220 & -.0251867 & .1084164 \\
\hline jul115 & .044681 & .0313513 & 1.43 & 0.157 & -.0173925 & .1067544 \\
\hline jul1631 & .0451409 & .0314732 & 1.43 & 0.154 & -.0171739 & .1074556 \\
\hline $\operatorname{aug} 115$ & .0404366 & .0301719 & 1.34 & 0.183 & -.0193017 & .1001749 \\
\hline atagor $\sim \mathrm{x}$ & .0016872 & .0108907 & 0.15 & 0.877 & -.0198756 & .02325 \\
\hline latago $\mathrm{x}$ & -.0009583 & .0008279 & -1.16 & 0.249 & -.0025975 & .0006809 \\
\hline cp2_matago x & -.0005606 & .0019459 & -0.29 & 0.774 & -.0044134 & .0032922 \\
\hline matag $x$ & $4.61 e-06$ & $4.85 e-06$ & 0.95 & 0.344 & $-4.99 e-06$ & .0000142 \\
\hline $\bar{d}$ mata $\sim x$ & -.0000539 & .0000969 & -0.56 & 0.579 & -.0002458 & .000138 \\
\hline _cons & .5940742 & .0224266 & 26.49 & 0.000 & .549671 & .6384774 \\
\hline
\end{tabular}


Tulia, TX

Linear regression

$\begin{array}{llr}\text { Number of obs } & = & 154 \\ \mathrm{~F}(29, \quad 124) & = & 7.04 \\ \text { Prob }>\text { F } & = & 0.0000 \\ \text { R-squared } & = & 0.5539 \\ \text { Root MSE } & = & .05623\end{array}$

\begin{tabular}{|c|c|c|c|c|c|c|}
\hline ndvi_tuliatx & Coef. & $\begin{array}{c}\text { Robust } \mathrm{HC} 3 \\
\text { Std. Err. }\end{array}$ & $t$ & $P>|t|$ & [95\% Conf. & Interval] \\
\hline year_1983 & .0281671 & .0420408 & 0.67 & 0.504 & -.0550434 & .1113777 \\
\hline year_1984 & -.0265483 & .0389118 & -0.68 & 0.496 & -.1035656 & .050469 \\
\hline year_1985 & -.0692729 & .0372197 & -1.86 & 0.065 & -.1429412 & .0043954 \\
\hline year_1986 & -.0246514 & .0347184 & -0.71 & 0.479 & -.0933688 & .0440661 \\
\hline year_1987 & -.0034911 & .03271 & -0.11 & 0.915 & -.0682333 & .0612511 \\
\hline year_1988 & .1186583 & .0344287 & 3.45 & 0.001 & .0505143 & .1868023 \\
\hline year_1989 & .0176907 & .0435862 & 0.41 & 0.686 & -.0685787 & .1039601 \\
\hline year_1990 & -.003792 & .0386092 & -0.10 & 0.922 & -.0802104 & .0726263 \\
\hline year_1991 & .0474187 & .0384983 & 1.23 & 0.220 & -.0287802 & .1236176 \\
\hline year_1992 & .0272243 & .0396144 & 0.69 & 0.493 & -.0511838 & .1056324 \\
\hline year_1993 & .026479 & .036532 & 0.72 & 0.470 & -.045828 & .098786 \\
\hline year_1994 & .0835271 & .0359481 & 2.32 & 0.022 & .0123757 & .1546785 \\
\hline year_1995 & .0552489 & .0378859 & 1.46 & 0.147 & -.019738 & .1302358 \\
\hline year_1996 & .0043562 & .0390198 & 0.11 & 0.911 & -.072875 & .0815873 \\
\hline year_1997 & .0892351 & .0417494 & 2.14 & 0.035 & .0066014 & .1718688 \\
\hline year_1998 & .010737 & .0401162 & 0.27 & 0.789 & -.0686641 & .0901382 \\
\hline year_1999 & .0564672 & .036304 & 1.56 & 0.122 & -.0153886 & .1283231 \\
\hline year_2000 & .0941618 & .0344401 & 2.73 & 0.007 & .0259951 & .1623285 \\
\hline year_2001 & .0063749 & .0441852 & 0.14 & 0.886 & -.08108 & .0938297 \\
\hline year_2002 & -.0716873 & .0469562 & -1.53 & 0.129 & -.1646268 & .0212522 \\
\hline year_2003 & -.0759806 & .0370862 & -2.05 & 0.043 & -.1493845 & -.0025766 \\
\hline $\operatorname{may} 1631$ & -.0043364 & .0210465 & -0.21 & 0.837 & -.0459933 & .0373204 \\
\hline jun115 & -.0017131 & .0188248 & -0.09 & 0.928 & -.0389727 & .0355464 \\
\hline jun 1630 & .0039367 & .019032 & 0.21 & 0.836 & -.033733 & .0416064 \\
\hline jul115 & .0185194 & .018483 & 1.00 & 0.318 & -.0180636 & .0551024 \\
\hline jul1631 & .0273157 & .0191807 & 1.42 & 0.157 & -.0106483 & .0652797 \\
\hline $\operatorname{aug} 115$ & .0390302 & .022496 & 1.73 & 0.085 & -.0054956 & .083556 \\
\hline cp_tuliatx & .0144195 & .0092522 & 1.56 & 0.122 & -.0038932 & .0327322 \\
\hline gdd_tuliatx & (dropped) & & & & & \\
\hline cp2_tuliatx & -.0018874 & .001537 & -1.23 & 0.222 & -.0049295 & .0011547 \\
\hline gdd2_tuliatx & (dropped) & & & & & \\
\hline cpgdd_tuli $\sim x$ & (dropped) & & & & & \\
\hline _cons & .3082402 & .0354237 & 8.70 & 0.000 & .2381269 & .3783535 \\
\hline
\end{tabular}




\section{Menomonie, TX}

Linear regression

$\begin{array}{llr}\text { Number of obs } & = & 154 \\ \mathrm{~F}(32, \text { 121) } & 18.10 \\ \text { Prob }>\text { F } & 0.0000 \\ \text { R-squared } & =0.7960 \\ \text { Root MSE } & = & .04416\end{array}$

\begin{tabular}{|c|c|c|c|c|c|c|}
\hline ndvi_menom e & Coef. & $\begin{array}{l}\text { Robust } \mathrm{HC} 3 \\
\text { Std. Err. }\end{array}$ & $t$ & $P>|t|$ & {$[95 \%$ Con } & Inte \\
\hline year_1983 & -.0364066 & .0132113 & -2.76 & 0.007 & -.0625619 & -.0102514 \\
\hline year_1984 & .0326507 & .0206862 & 1.58 & 0.117 & -.0083031 & .0736045 \\
\hline year_1985 & -.0023887 & .0327482 & -0.07 & 0.942 & -.0672224 & .062445 \\
\hline year_1986 & -.0117229 & .0161278 & -0.73 & 0.469 & -.0436521 & .0202063 \\
\hline year_1987 & -.0221536 & .0147284 & -1.50 & 0.135 & -.0513122 & .0070051 \\
\hline year_1988 & -.0275173 & .015291 & -1.80 & 0.074 & -.0577898 & .0027552 \\
\hline year_1989 & .0159809 & .0210513 & 0.76 & 0.449 & -.0256956 & .0576574 \\
\hline year_1990 & -.018443 & .0176395 & -1.05 & 0.298 & -.053365 & .0164791 \\
\hline year_1991 & -.0167713 & .0171748 & -0.98 & 0.331 & -.0507733 & .0172308 \\
\hline year_1992 & .0312075 & .0156603 & 1.99 & 0.049 & .0002039 & .0622112 \\
\hline year_1993 & -.0341212 & .0242941 & -1.40 & 0.163 & -.0822178 & .0139754 \\
\hline year_1994 & .0247872 & .0124609 & 1.99 & 0.049 & .0001176 & .0494568 \\
\hline year_1995 & -.010081 & .0168426 & -0.60 & 0.551 & -.0434254 & .0232634 \\
\hline year_1996 & .006224 & .0246681 & 0.25 & 0.801 & -.042613 & .055061 \\
\hline year_1997 & -.0498296 & .0287981 & -1.73 & 0.086 & -.1068429 & .0071838 \\
\hline year_1998 & -.0366353 & .0377293 & -0.97 & 0.333 & -.1113304 & .0380598 \\
\hline year_1999 & -.0733821 & .0302226 & -2.43 & 0.017 & -.1332156 & -.0135486 \\
\hline year_2000 & -.0272206 & .0145379 & -1.87 & 0.064 & -.0560023 & .001561 \\
\hline year_2001 & -.0129338 & .012519 & -1.03 & 0.304 & -.0377183 & .0118508 \\
\hline year_2002 & -.0239319 & .0157844 & -1.52 & 0.132 & -.0551813 & .0073176 \\
\hline year_2003 & -.0469591 & .012888 & -3.64 & 0.000 & -.0724742 & -.021444 \\
\hline maȳ1631 & .0820117 & .0158044 & 5.19 & 0.000 & .0507226 & .1133008 \\
\hline jun 115 & .1297563 & .0195742 & 6.63 & 0.000 & .0910039 & .1685087 \\
\hline jun 1630 & .1629365 & .0183179 & 8.89 & 0.000 & .1266713 & .1992017 \\
\hline jul115 & .1871276 & .0194565 & 9.62 & 0.000 & .1486084 & .2256467 \\
\hline jul1631 & .2066439 & .0208285 & 9.92 & 0.000 & .1654083 & .2478794 \\
\hline $\operatorname{aug} 115$ & .1981305 & .0183012 & 10.83 & 0.000 & .1618985 & .2343624 \\
\hline nomonie & .0102678 & .0102381 & 1.00 & 0.318 & -.0100012 & .0305368 \\
\hline menonie & .0008236 & .0006795 & 1.21 & 0.228 & -.0005216 & .0021687 \\
\hline cp2_menemo e & -.0018679 & .0016214 & -1.15 & 0.252 & -.005078 & .0013422 \\
\hline menem i & $-8.07 e-06$ & $7.68 e-06$ & -1.05 & 0.295 & -.0000233 & $7.13 e-06$ \\
\hline $\bar{d}$ meno i & -.0000293 & .0001199 & -0.24 & 0.807 & -.0002667 & .000208 \\
\hline _cons & .5483372 & .0165771 & 33.08 & 0.000 & .5155185 & .581156 \\
\hline
\end{tabular}




\section{Dalton, WI}

Linear regression

$\begin{array}{llr}\text { Number of obs } & = & 154 \\ \mathrm{~F}(32, \text { 121) } & 18.87 \\ \text { Prob }>\text { F } & 0.0000 \\ \text { R-squared } & =0.8330 \\ \text { Root MSE } & = & .04538\end{array}$

\begin{tabular}{|c|c|c|c|c|c|c|}
\hline ndvi_dalto i & Coef. & $\begin{array}{c}\text { Robust HC3 } \\
\text { Std. Err. }\end{array}$ & $t$ & $P>|t|$ & [ $95 \%$ Conf. & ral] \\
\hline year_1983 & -.0042897 & .0260234 & -0.16 & 0.869 & -.0558099 & .0472305 \\
\hline year_1984 & .0073105 & .0184716 & 0.40 & 0.693 & -.0292589 & .0438798 \\
\hline year_1985 & .02858 & .0268999 & 1.06 & 0.290 & -.0246754 & .0818355 \\
\hline year_1986 & .0586936 & .0184171 & 3.19 & 0.002 & .022232 & .0951551 \\
\hline year_1987 & .0353071 & .0237498 & 1.49 & 0.140 & -.0117119 & .0823262 \\
\hline year_1988 & .0071359 & .0295398 & 0.24 & 0.810 & -.051346 & .0656177 \\
\hline year_1989 & -.0265031 & .0205167 & -1.29 & 0.199 & -.0671214 & .0141151 \\
\hline year_1990 & .0142635 & .0216989 & 0.66 & 0.512 & -.0286952 & .0572222 \\
\hline year_1991 & .0758968 & .0272513 & 2.79 & 0.006 & .0219456 & .1298479 \\
\hline year_1992 & .0320593 & .0226946 & 1.41 & 0.160 & -.0128707 & .0769893 \\
\hline year_1993 & .0226808 & .0168362 & 1.35 & 0.180 & -.010651 & .0560125 \\
\hline year_1994 & .010693 & .0265981 & 0.40 & 0.688 & -.041965 & .063351 \\
\hline year_1995 & .0122155 & .0216467 & 0.56 & 0.574 & -.0306398 & .0550707 \\
\hline year_1996 & .00229 & .0289893 & 0.08 & 0.937 & -.055102 & .0596819 \\
\hline year_1997 & .0107551 & .0243647 & 0.44 & 0.660 & -.0374813 & .0589915 \\
\hline year_1998 & .0474545 & .0270006 & 1.76 & 0.081 & -.0060004 & .1009093 \\
\hline year_1999 & -.0282877 & .0402861 & -0.70 & 0.484 & -.1080446 & .0514692 \\
\hline year_2000 & -.0023978 & .0186308 & -0.13 & 0.898 & -.0392824 & .0344868 \\
\hline year_2001 & .0258172 & .0317652 & 0.81 & 0.418 & -.0370704 & .0887049 \\
\hline year_2002 & -.0025823 & .0194891 & -0.13 & 0.895 & -.0411661 & .0360016 \\
\hline year_2003 & -.0033031 & .0200835 & -0.16 & 0.870 & -.0430637 & .0364575 \\
\hline $\operatorname{may} 1631$ & .0830623 & .0176487 & 4.71 & 0.000 & .0481221 & .1180024 \\
\hline jun115 & .1604417 & .0177393 & 9.04 & 0.000 & .1253221 & .1955613 \\
\hline jun 1630 & .2033969 & .0169784 & 11.98 & 0.000 & .1697836 & .2370103 \\
\hline jul115 & .2374799 & .0198115 & 11.99 & 0.000 & .1982578 & .2767021 \\
\hline jul1631 & .238948 & .0195571 & 12.22 & 0.000 & .2002295 & .2776665 \\
\hline $\operatorname{aug} 115$ & .2402543 & .016627 & 14.45 & 0.000 & .2073367 & .2731718 \\
\hline daltonwi & -.0154388 & .0109624 & -1.41 & 0.162 & -.0371418 & .0062641 \\
\hline gdd_daltonwi & -.0000181 & .0008089 & -0.02 & 0.982 & -.0016194 & .0015833 \\
\hline cp2_daltonwi & .0017872 & .0016913 & 1.06 & 0.293 & -.0015612 & .0051356 \\
\hline gdd 2 _dalto i & $-3.64 e-06$ & $9.04 e-06$ & -0.40 & 0.688 & -.0000215 & .0000143 \\
\hline cpgdd_dalt $i$ & .0000745 & .0002253 & 0.33 & 0.742 & -.0003716 & .0005206 \\
\hline _cons & .5494448 & .0218259 & 25.17 & 0.000 & .5062347 & .592655 \\
\hline
\end{tabular}




\section{Arlington, WI}

Linear regression

$\begin{array}{llr}\text { Number of obs } & = & 154 \\ \text { F }(32,121) & 13.71 \\ \text { Prob F } & =0.0000 \\ \text { R-squared } & =0.7685 \\ \text { Root MSE } & = & .06115\end{array}$

\begin{tabular}{|c|c|c|c|c|c|c|}
\hline ndvi_arlin i & Coef. & $\begin{array}{c}\text { Robust } \mathrm{HC} 3 \\
\text { Std. Err. }\end{array}$ & $t$ & $P>|t|$ & [ $95 \%$ Conf. & Interval] \\
\hline year_1983 & -.0311812 & .0309049 & -1.01 & 0.315 & -.0923656 & .0300033 \\
\hline year_1984 & .0305942 & .0285047 & 1.07 & 0.285 & -.0258384 & .0870268 \\
\hline year_1985 & .0447327 & .0176595 & 2.53 & 0.013 & .009771 & .0796943 \\
\hline year_1986 & .0525057 & .0184648 & 2.84 & 0.005 & .0159497 & .0890617 \\
\hline year_1987 & .0469037 & .0358569 & 1.31 & 0.193 & -.0240845 & .1178919 \\
\hline year_1988 & .0520586 & .0366486 & 1.42 & 0.158 & -.0204969 & .1246141 \\
\hline year_1989 & .0101322 & .0261366 & 0.39 & 0.699 & -.0416122 & .0618765 \\
\hline year_1990 & -.0194676 & .0294006 & -0.66 & 0.509 & -.0776739 & .0387387 \\
\hline year_1991 & .041642 & .0204218 & 2.04 & 0.044 & .0012116 & .0820724 \\
\hline year_1992 & .0098376 & .0189206 & 0.52 & 0.604 & -.0276208 & .047296 \\
\hline year_1993 & -.004338 & .0251164 & -0.17 & 0.863 & -.0540625 & .0453865 \\
\hline year_1994 & .0141119 & .0468599 & 0.30 & 0.764 & -.0786596 & .1068834 \\
\hline year_1995 & .0061696 & .0249704 & 0.25 & 0.805 & -.043266 & .0556052 \\
\hline year_1996 & .0232164 & .022092 & 1.05 & 0.295 & -.0205205 & .0669533 \\
\hline year_1997 & .0107037 & .0307613 & 0.35 & 0.728 & -.0501963 & .0716037 \\
\hline year_1998 & -.0072959 & .0298606 & -0.24 & 0.807 & -.0664128 & .0518211 \\
\hline year_1999 & -.0680142 & .0586715 & -1.16 & 0.249 & -.1841699 & .0481415 \\
\hline year_2000 & .0090389 & .0192626 & 0.47 & 0.640 & -.0290965 & .0471743 \\
\hline year_2001 & -.0374038 & .0333927 & -1.12 & 0.265 & -.1035135 & .0287058 \\
\hline year_2002 & -.0260043 & .0347651 & -0.75 & 0.456 & -.094831 & .0428224 \\
\hline year_2003 & -.0264354 & .0373229 & -0.71 & 0.480 & -.1003259 & .0474552 \\
\hline $\operatorname{may} 1631$ & .035012 & .0188505 & 1.86 & 0.066 & -.0023076 & .0723316 \\
\hline jun115 & .1048855 & .0205967 & 5.09 & 0.000 & .0641089 & .1456622 \\
\hline jun 1630 & .165954 & .0178222 & 9.31 & 0.000 & .1306703 & .2012378 \\
\hline jul115 & .2352101 & .0205394 & 11.45 & 0.000 & .194547 & .2758732 \\
\hline jul1631 & .2524576 & .0257254 & 9.81 & 0.000 & .2015274 & .3033878 \\
\hline $\operatorname{aug} 115$ & .2608404 & .0191842 & 13.60 & 0.000 & .2228603 & .2988206 \\
\hline rlingt i & -.0131139 & .0129877 & -1.01 & 0.315 & -.0388266 & .0125987 \\
\hline gdd_arling i & -.0010063 & .0010228 & -0.98 & 0.327 & -.0030313 & .0010187 \\
\hline cp2_arling i & .0012638 & .0015249 & 0.83 & 0.409 & -.0017552 & .0042827 \\
\hline gdd $\overline{2}$ arlin i & $-2.96 e-06$ & $7.48 e-06$ & -0.40 & 0.693 & -.0000178 & .0000118 \\
\hline cpgdō_arli i & .0003149 & .0003466 & 0.91 & 0.365 & -.0003713 & .001001 \\
\hline _cons & .5203711 & .023418 & 22.22 & 0.000 & .4740091 & .5667332 \\
\hline
\end{tabular}


Luck, WI

Linear regression

$\begin{array}{llr}\text { Number of obs } & = & 154 \\ \text { F }(32,121) & 15.81 \\ \text { Prob F } & =0000 \\ \text { R-squared } & =0.8308 \\ \text { Root MSE } & = & .05169\end{array}$

\begin{tabular}{|c|c|c|c|c|c|c|}
\hline ndvi_luckwi & Coef. & $\begin{array}{l}\text { Robust } \mathrm{HC} 3 \\
\text { Std. Err. }\end{array}$ & $t$ & $P>|t|$ & [95\% Conf. & Interval] \\
\hline year_1983 & -.0568647 & .0233613 & -2.43 & 0.016 & -.1031146 & -.0106149 \\
\hline year-1984 & .0240334 & .0147177 & 1.63 & 0.105 & -.0051041 & .053171 \\
\hline year 1985 & -.0088068 & .0339522 & -0.26 & 0.796 & -.0760242 & .0584106 \\
\hline year-1986 & .0040527 & .0157635 & 0.26 & 0.798 & -.0271554 & .0352608 \\
\hline year_1987 & -.0754585 & .0358281 & -2.11 & 0.037 & -.1463897 & -.0045273 \\
\hline year-1988 & -.0294435 & .0335509 & -0.88 & 0.382 & -.0958663 & .0369793 \\
\hline year_1989 & -.0449946 & .0257859 & -1.74 & 0.084 & -.0960447 & .0060555 \\
\hline year-1990 & -.0670903 & .0119819 & -5.60 & 0.000 & -.0908116 & -.043369 \\
\hline year_1991 & -.0283138 & .0221296 & -1.28 & 0.203 & -.0721252 & .0154975 \\
\hline year_1992 & .0177597 & .0235007 & 0.76 & 0.451 & -.028766 & .0642855 \\
\hline year_1993 & -.0259828 & .0268079 & -0.97 & 0.334 & -.0790561 & .0270904 \\
\hline year_1994 & -.0165821 & .0144502 & -1.15 & 0.253 & -.04519 & .0120259 \\
\hline year_1995 & .0048747 & .0260798 & 0.19 & 0.852 & -.0467572 & .0565066 \\
\hline year_1996 & .0017983 & .0251399 & 0.07 & 0.943 & -.0479728 & .0515694 \\
\hline year_1997 & -.0196098 & .0295734 & -0.66 & 0.509 & -.0781581 & .0389385 \\
\hline year_1998 & -.0807289 & .0403516 & -2.00 & 0.048 & -.1606156 & -.0008421 \\
\hline year_1999 & -.0459335 & .0258602 & -1.78 & 0.078 & -.0971306 & .0052636 \\
\hline year_2000 & -.0673836 & .0173153 & -3.89 & 0.000 & -.1016639 & -.0331034 \\
\hline year-2001 & -.0397917 & .0223295 & -1.78 & 0.077 & -.0839989 & .0044155 \\
\hline year_2002 & -.0689366 & .027611 & -2.50 & 0.014 & -.1235999 & -.0142733 \\
\hline year_2003 & -.0804032 & .0254604 & -3.16 & 0.002 & -.1308087 & -.0299977 \\
\hline $\operatorname{may} 1631$ & .1430576 & .0225114 & 6.35 & 0.000 & .0984904 & .1876247 \\
\hline jun115 & .2216417 & .0233305 & 9.50 & 0.000 & .1754529 & .2678306 \\
\hline jun 1630 & .2692031 & .0214092 & 12.57 & 0.000 & .226818 & .3115882 \\
\hline jul115 & .2796405 & .0228158 & 12.26 & 0.000 & .2344705 & .3248105 \\
\hline jul1631 & .2756873 & .0218622 & 12.61 & 0.000 & .2324053 & .3189692 \\
\hline $\operatorname{aug} 115$ & .2613755 & .0225566 & 11.59 & 0.000 & .2167187 & .3060323 \\
\hline cp_luckwi & -.017535 & .0138936 & -1.26 & 0.209 & -.045041 & .0099711 \\
\hline gdd_luckwi & .0002113 & .0011526 & 0.18 & 0.855 & -.0020707 & .0024932 \\
\hline cp2_luckwi & .002957 & .0024031 & 1.23 & 0.221 & -.0018004 & .0077145 \\
\hline gdd2_luckwi & -.0000137 & .0000154 & -0.89 & 0.373 & -.0000442 & .0000167 \\
\hline cpgdd_luckwi & .0002343 & .0003208 & 0.73 & 0.467 & -.0004009 & .0008695 \\
\hline _cons & .592426 & .0262462 & 22.57 & 0.000 & .5404647 & .6443873 \\
\hline
\end{tabular}




\section{Shippensburg, PA}

Linear regression

$\begin{array}{llr}\text { Number of obs } & = & 154 \\ \mathrm{~F}(32, \quad 121) & 10.09 \\ \text { Prob }>\text { F } & =0000 \\ \text { R-squared } & =0.5150 \\ \text { Root MSE } & = & .06938\end{array}$

\begin{tabular}{|c|c|c|c|c|c|c|}
\hline ndvi_shipp a & Coef. & $\begin{array}{l}\text { Robust } \mathrm{HC} 3 \\
\text { Std. Err. }\end{array}$ & $t$ & $P>|t|$ & [95\% Conf. & Int \\
\hline year_1983 & -.013201 & .0432358 & -0.31 & 0.761 & -.0987976 & .0723956 \\
\hline year_1984 & -.0715554 & .033183 & -2.16 & 0.033 & -.13725 & -.0058609 \\
\hline year_1985 & .0507484 & .0319017 & 1.59 & 0.114 & -.0124094 & .1139063 \\
\hline year_1986 & -.0226963 & .0288543 & -0.79 & 0.433 & -.079821 & .0344283 \\
\hline year_1987 & .0022027 & .0435882 & 0.05 & 0.960 & -.0840917 & .0884971 \\
\hline year_1988 & -.0642991 & .0273248 & -2.35 & 0.020 & -.1183957 & -.0102025 \\
\hline year_1989 & -.0520266 & .0339565 & -1.53 & 0.128 & -.1192525 & .0151993 \\
\hline year_1990 & .0895334 & .0449253 & 1.99 & 0.049 & .0005919 & .1784749 \\
\hline year_1991 & -.0599186 & .0563339 & -1.06 & 0.290 & -.1714465 & .0516092 \\
\hline year_1992 & .0103299 & .0366057 & 0.28 & 0.778 & -.0621408 & .0828006 \\
\hline year_1993 & .0388453 & .0334251 & 1.16 & 0.247 & -.0273286 & .1050191 \\
\hline year_1994 & .0458523 & .0353188 & 1.30 & 0.197 & -.0240705 & .1157751 \\
\hline year_1995 & -.0934135 & .0394872 & -2.37 & 0.020 & -.1715888 & -.0152381 \\
\hline year_1996 & .0067701 & .0293786 & 0.23 & 0.818 & -.0513925 & .0649327 \\
\hline year_1997 & .0823736 & .0267561 & 3.08 & 0.003 & .0294027 & .1353444 \\
\hline year_1998 & .0185231 & .029813 & 0.62 & 0.536 & -.0404995 & .0775458 \\
\hline year_1999 & -.0755848 & .0652089 & -1.16 & 0.249 & -.204683 & .0535133 \\
\hline year_2000 & .0048905 & .0247741 & 0.20 & 0.844 & -.0441564 & .0539374 \\
\hline year_2001 & .0523029 & .0218761 & 2.39 & 0.018 & .0089935 & .0956123 \\
\hline year_2002 & .022422 & .020935 & 1.07 & 0.286 & -.0190244 & .0638684 \\
\hline year_2003 & -.0574766 & .0345785 & -1.66 & 0.099 & -.1259338 & .0109807 \\
\hline $\operatorname{may} 1631$ & .0586347 & .0195328 & 3.00 & 0.003 & .0199644 & .0973051 \\
\hline jun115 & .0789695 & .0206427 & 3.83 & 0.000 & .0381018 & .1198372 \\
\hline jun 1630 & .103363 & .0194369 & 5.32 & 0.000 & .0648825 & .1418436 \\
\hline jul115 & .1165226 & .0252435 & 4.62 & 0.000 & .0665464 & .1664987 \\
\hline jul1631 & .1143348 & .0223579 & 5.11 & 0.000 & .0700714 & .1585983 \\
\hline $\operatorname{aug} 115$ & .1105026 & .0213807 & 5.17 & 0.000 & .0681738 & .1528314 \\
\hline hippen a & -.0062832 & .014385 & -0.44 & 0.663 & -.0347621 & .0221957 \\
\hline gdd_shippe a & .0027244 & .0075676 & 0.36 & 0.719 & -.0122575 & .0177064 \\
\hline cp2_shippe a & .0027755 & .002243 & 1.24 & 0.218 & -.0016651 & .0072161 \\
\hline gdd2__shipp a & -.0000742 & .0001425 & -0.52 & 0.603 & -.0003564 & .0002079 \\
\hline pgdd__shep a & -.000934 & .0016377 & -0.57 & 0.570 & -.0041764 & .0023083 \\
\hline _cons & .6750596 & .0231448 & 29.17 & 0.000 & .6292383 & .7208809 \\
\hline
\end{tabular}


Sellingsgrove, PA

Linear regression

$\begin{array}{llr}\text { Number of obs } & & 154 \\ \text { F }(29, \quad 124) & 3.58 \\ \text { Prob F } & =0000 \\ \text { R-squared } & =0.4636 \\ \text { Root MSE } & = & .04674\end{array}$

\begin{tabular}{|c|c|c|c|c|c|c|}
\hline ndvi_selli a & Coef. & $\begin{array}{l}\text { Robust HC3 } \\
\text { Std. Err. }\end{array}$ & $t$ & $P>|t|$ & [95\% Conf. & Inte \\
\hline $\begin{array}{l}\text { year_1983 } \\
\text { year_1984 }\end{array}$ & $\begin{array}{r}.015246 \\
-.0092806\end{array}$ & $\begin{array}{l}.0172762 \\
.0281678\end{array}$ & $\begin{array}{r}0.88 \\
-0.33\end{array}$ & $\begin{array}{l}0.379 \\
0.742\end{array}$ & $\begin{array}{l}-.0189484 \\
-.0650325\end{array}$ & $\begin{array}{l}.0494404 \\
.0464713\end{array}$ \\
\hline year_1985 & .0089963 & .0153983 & 0.58 & 0.560 & -.0214812 & .0394737 \\
\hline year_1986 & -.0099774 & .0222544 & -0.45 & 0.655 & -.0540251 & .0340704 \\
\hline year_1987 & .0154493 & .0203132 & 0.76 & 0.448 & -.0247561 & .0556547 \\
\hline year_1988 & -.0392889 & .0230051 & -1.71 & 0.090 & -.0848226 & .0062447 \\
\hline year_1989 & -.0104979 & .0263638 & -0.40 & 0.691 & -.0626791 & .0416834 \\
\hline year_1990 & .0379548 & .0203726 & 1.86 & 0.065 & -.0023683 & .0782779 \\
\hline year_1991 & .0410676 & .0179075 & 2.29 & 0.024 & .0056237 & .0765116 \\
\hline year_1992 & .0272 & .0206277 & 1.32 & 0.190 & -.013628 & .068028 \\
\hline year_1993 & -.0085502 & .0155091 & -0.55 & 0.582 & -.039247 & .0221467 \\
\hline year_1994 & .0168422 & .0297888 & 0.57 & 0.573 & -.0421182 & .0758026 \\
\hline year_1995 & -.0459423 & .0414172 & -1.11 & 0.269 & -.1279186 & .036034 \\
\hline year_1996 & .0105766 & .0223779 & 0.47 & 0.637 & -.0337154 & .0548687 \\
\hline year_1997 & .0016892 & .0193662 & 0.09 & 0.931 & -.0366419 & .0400203 \\
\hline year_1998 & .0035236 & .0208343 & 0.17 & 0.866 & -.0377134 & .0447605 \\
\hline year_1999 & -.0745858 & .0372895 & -2.00 & 0.048 & -.1483921 & -.0007795 \\
\hline year_2000 & -.0052366 & .0178661 & -0.29 & 0.770 & -.0405986 & .0301255 \\
\hline year_2001 & -.0108769 & .0215467 & -0.50 & 0.615 & -.0535238 & .03177 \\
\hline year_2002 & .0078806 & .0186382 & 0.42 & 0.673 & -.0290097 & .0447709 \\
\hline year_2003 & -.023636 & .0182139 & -1.30 & 0.197 & -.0596864 & .0124144 \\
\hline $\operatorname{may} 1631$ & .0528045 & .0157605 & 3.35 & 0.001 & .02161 & .083999 \\
\hline jun115 & .0637119 & .0143691 & 4.43 & 0.000 & .0352714 & .0921523 \\
\hline jun 1630 & .0698435 & .0182156 & 3.83 & 0.000 & .0337897 & .1058972 \\
\hline jul115 & .0798786 & .01549 & 5.16 & 0.000 & .0492195 & .1105376 \\
\hline jul1631 & .0940699 & .0143497 & 6.56 & 0.000 & .0656677 & .122472 \\
\hline $\operatorname{aug} 115$ & .085483 & .0138894 & 6.15 & 0.000 & .0579921 & .112974 \\
\hline $\begin{array}{l}\text { cp_sellins a } \\
\text { gdd_sellin a }\end{array}$ & $\begin{array}{r}-.0113537 \\
\text { (dropped) }\end{array}$ & .012069 & -0.94 & 0.349 & -.0352416 & .0125341 \\
\hline $\begin{array}{l}\text { cp2_sellin a } \\
\text { gdd2_selli a } \\
\text { cpgdd_sell a }\end{array}$ & $\begin{array}{l}.0012892 \\
\text { (dropped) } \\
\text { (dropped) }\end{array}$ & .0024541 & 0.53 & 0.600 & -.0035682 & .0061466 \\
\hline cons & .657795 & .0193304 & 34.03 & 0.000 & .6195349 & .6960552 \\
\hline
\end{tabular}




\section{Montrose, PA}

Linear regression

$\begin{array}{llr}\text { Number of obs } & & 154 \\ \mathrm{~F}(29, \quad 124) & = & 8.57 \\ \text { Prob }>\text { F } & = & 0.0000 \\ \text { R-squared } & =0.6463 \\ \text { Root MSE } & = & .05773\end{array}$

\begin{tabular}{|c|c|c|c|c|c|c|}
\hline ndvi_montr a & Coef. & $\begin{array}{l}\text { Robust } \mathrm{HC} 3 \\
\text { Std. Err. }\end{array}$ & $t$ & $P>|t|$ & {$\left[\begin{array}{l}95 \% \\
\text { Conf. }\end{array}\right.$} & Interval] \\
\hline year_1983 & .0235783 & .0391134 & 0.60 & 0.548 & -.0538382 & .1009947 \\
\hline year_1984 & .0194378 & .0415136 & 0.47 & 0.640 & -.0627293 & .1016048 \\
\hline year_1985 & .0933952 & .030759 & 3.04 & 0.003 & .0325146 & .1542758 \\
\hline year_1986 & .0232428 & .0390237 & 0.60 & 0.553 & -.0539959 & .1004816 \\
\hline year_1987 & .0612605 & .031616 & 1.94 & 0.055 & -.0013164 & .1238374 \\
\hline year_1988 & .0128387 & .0322579 & 0.40 & 0.691 & -.0510088 & .0766862 \\
\hline year_1989 & -.0659158 & .0352401 & -1.87 & 0.064 & -.1356657 & .0038342 \\
\hline year_1990 & -.0119828 & .0294022 & -0.41 & 0.684 & -.0701779 & .0462124 \\
\hline year_1991 & .0600806 & .0306337 & 1.96 & 0.052 & -.0005519 & .1207132 \\
\hline year_1992 & .0682436 & .0331164 & 2.06 & 0.041 & .002697 & .1337903 \\
\hline year_1993 & .0202391 & .0346355 & 0.58 & 0.560 & -.0483143 & .0887925 \\
\hline year_1994 & .0088343 & .0430743 & 0.21 & 0.838 & -.0764218 & .0940905 \\
\hline year_1995 & .0209776 & .0412 & 0.51 & 0.612 & -.0605689 & .102524 \\
\hline year_1996 & .0253779 & .0312038 & 0.81 & 0.418 & -.0363832 & .0871389 \\
\hline year_1997 & .0333402 & .0377026 & 0.88 & 0.378 & -.0412838 & .1079642 \\
\hline year_1998 & .0032642 & .0325129 & 0.10 & 0.920 & -.0610879 & .0676162 \\
\hline year_1999 & -.0584305 & .040384 & -1.45 & 0.150 & -.1383617 & .0215008 \\
\hline year_2000 & -.0163173 & .0289766 & -0.56 & 0.574 & -.0736702 & .0410356 \\
\hline year_2001 & -.0010529 & .0346527 & -0.03 & 0.976 & -.0696403 & .0675345 \\
\hline year_2002 & -.0133537 & .0327275 & -0.41 & 0.684 & -.0781306 & .0514231 \\
\hline year_2003 & -.0451561 & .0436118 & -1.04 & 0.302 & -.1314761 & .041164 \\
\hline may1631 & .0875197 & .0223563 & 3.91 & 0.000 & .0432704 & .131769 \\
\hline jun115 & .1428988 & .0185489 & 7.70 & 0.000 & .1061853 & .1796122 \\
\hline jun 1630 & .1598138 & .0203191 & 7.87 & 0.000 & .1195966 & .2000311 \\
\hline jul115 & .1687001 & .0165353 & 10.20 & 0.000 & .1359722 & .201428 \\
\hline jul1631 & .1587688 & .0185996 & 8.54 & 0.000 & .1219551 & .1955826 \\
\hline aug115 & .1650097 & .0200229 & 8.24 & 0.000 & .1253787 & .2046407 \\
\hline montros a & .0058661 & .0132359 & 0.44 & 0.658 & -.0203315 & .0320638 \\
\hline gdd_montro a & (dropped) & & & & & \\
\hline cp2 montro a & -.0018332 & .0023135 & -0.79 & 0.430 & -.0064122 & .0027457 \\
\hline $\begin{array}{l}\text { gdd } 2 \text { montr } \sim a \\
\text { cpgdd mont } \sim \text { a }\end{array}$ & (dropped) & & & & & \\
\hline cons & .6416851 & .0317556 & 20.21 & 0.000 & .5788319 & .7045383 \\
\hline
\end{tabular}


Lagrange, WY

Linear regression

$\begin{array}{llr}\text { Number of obs } & = & 153 \\ \mathrm{~F}(32, \quad 120) & = & 5.48 \\ \text { Prob }>\text { F } & = & 0.0000 \\ \text { R-squared } & =0.5516 \\ \text { Root MSE } & = & .05359\end{array}$

\begin{tabular}{|c|c|c|c|c|c|c|}
\hline ndvi_lagra y & Coef. & $\begin{array}{l}\text { Robust } \mathrm{HC} 3 \\
\text { Std. Err. }\end{array}$ & $t$ & $P>|t|$ & [95\% Conf. & Inter \\
\hline year_1983 & .0459198 & .0294397 & 1.56 & 0.121 & -.0123687 & .1042083 \\
\hline year_1984 & -.007125 & .0347048 & -0.21 & 0.838 & -.075838 & .0615881 \\
\hline year_1985 & -.0251933 & .0371397 & -0.68 & 0.499 & -.0987273 & .0483408 \\
\hline year_1986 & -.0397831 & .0338362 & -1.18 & 0.242 & -.1067765 & .0272103 \\
\hline year_1987 & .0222274 & .0283283 & 0.78 & 0.434 & -.0338606 & .0783155 \\
\hline year_1988 & .0247396 & .0348443 & 0.71 & 0.479 & -.0442497 & .093729 \\
\hline year_1989 & -.0280631 & .0399198 & -0.70 & 0.483 & -.1071016 & .0509753 \\
\hline year_1990 & .0306483 & .0372534 & 0.82 & 0.412 & -.0431109 & .1044075 \\
\hline year_1991 & .047113 & .0334515 & 1.41 & 0.162 & -.0191187 & .1133447 \\
\hline year-1992 & .0113872 & .0371686 & 0.31 & 0.760 & -.062204 & .0849784 \\
\hline year_1993 & .0649049 & .0311551 & 2.08 & 0.039 & .0032201 & .1265898 \\
\hline year_1994 & .0787694 & .0359433 & 2.19 & 0.030 & .0076042 & .1499346 \\
\hline year_1995 & .04049 & .0341644 & 1.19 & 0.238 & -.0271532 & .1081332 \\
\hline year_1996 & .0658429 & .0344924 & 1.91 & 0.059 & -.0024496 & .1341354 \\
\hline year_1997 & .0175426 & .0344237 & 0.51 & 0.611 & -.0506139 & .085699 \\
\hline year_1998 & .0396444 & .0404991 & 0.98 & 0.330 & -.0405409 & .1198297 \\
\hline year_1999 & .0391171 & .0478367 & 0.82 & 0.415 & -.0555962 & .1338305 \\
\hline year_2000 & .032246 & .0340106 & 0.95 & 0.345 & -.0350926 & .0995846 \\
\hline year_2001 & .0492492 & .0504304 & 0.98 & 0.331 & -.0505995 & .1490979 \\
\hline year_2002 & -.0392638 & .0403465 & -0.97 & 0.332 & -.119147 & .0406195 \\
\hline year_2003 & .0913129 & .0388875 & 2.35 & 0.021 & .0143184 & .1683074 \\
\hline maȳ1631 & .0495859 & .0199815 & 2.48 & 0.014 & .0100239 & .089148 \\
\hline jun115 & .0907997 & .0191061 & 4.75 & 0.000 & .052971 & .1286284 \\
\hline jun 1630 & .1100974 & .0202931 & 5.43 & 0.000 & .0699185 & .1502763 \\
\hline jul115 & .1414999 & .0212413 & 6.66 & 0.000 & .0994435 & .1835562 \\
\hline jul1631 & .1405685 & .0234287 & 6.00 & 0.000 & .0941814 & .1869557 \\
\hline $\operatorname{aug} 115$ & .123032 & .0241615 & 5.09 & 0.000 & .0751939 & .1708701 \\
\hline agrang y & -.0128408 & .0160294 & -0.80 & 0.425 & -.0445779 & .0188963 \\
\hline gdd_lagran $\sim y$ & -.0006562 & .000717 & -0.92 & 0.362 & -.0020757 & .0007634 \\
\hline cp2_lagran y & .0021316 & .0037566 & 0.57 & 0.571 & -.0053061 & .0095694 \\
\hline dd'2_lagra y & $4.05 e-07$ & $5.08 e-06$ & 0.08 & 0.937 & $-9.66 e-06$ & .0000105 \\
\hline $\bar{d}$ _lagr $\sim y$ & -.0000991 & .0002389 & -0.42 & 0.679 & -.0005721 & .0003738 \\
\hline _cons & .3667029 & .0318479 & 11.51 & 0.000 & .3036462 & .4297596 \\
\hline
\end{tabular}




\section{APPENDIX B}

Regression Results for Yields, NDVI, and Meteorological Variables

\section{$\underline{\text { Regression results for corn yields }}$}

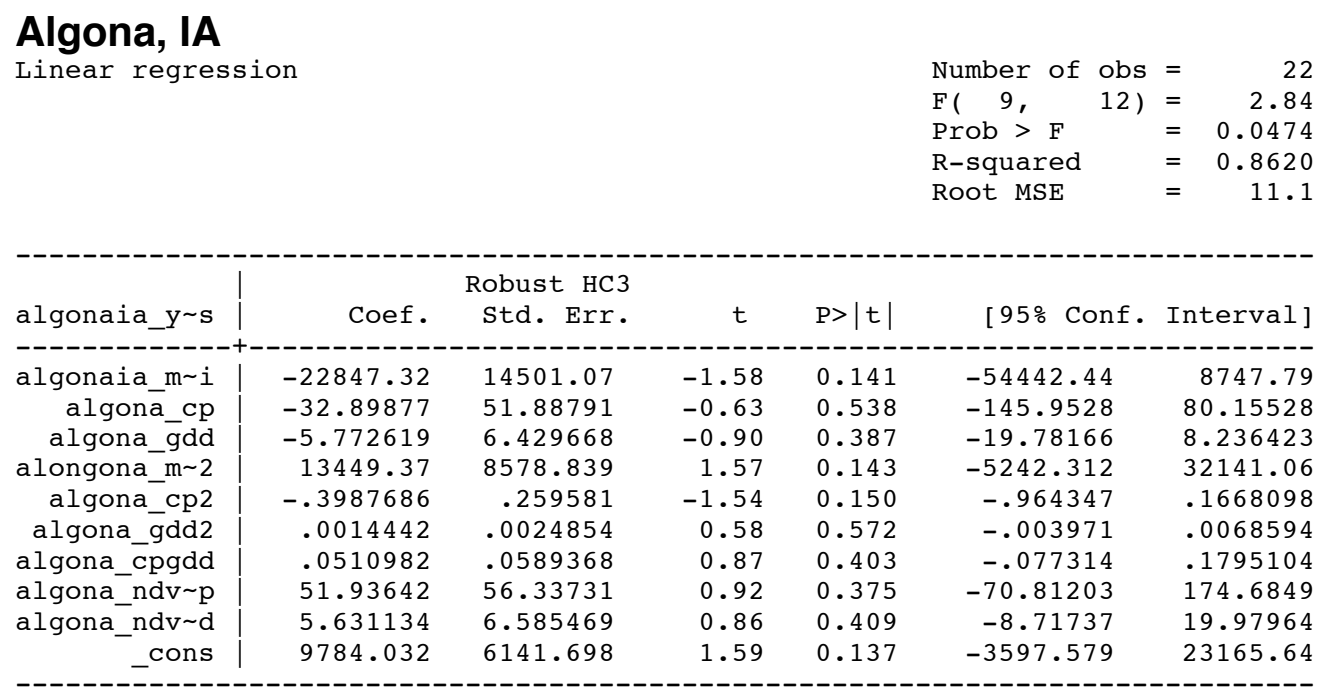

\section{Rockrapids, IA}

Linear regression

$\begin{array}{llr}\text { Number of obs } & & 22 \\ \text { F }(5, \quad 16) & 3.63 \\ \text { Prob }>\text { F } & =0220 \\ \text { R-squared } & =0.5286 \\ \text { Root MSE } & =20.064\end{array}$

\begin{tabular}{|c|c|c|c|c|c|c|}
\hline & & Robust $\mathrm{HC} 3$ & & & & \\
\hline rockrapids $\sim$ s & Coef. & Std. Err. & $t$ & $P>|t|$ & [95\% Conf. & Interval] \\
\hline rockrapids $\sim$ & 18664.42 & 14984.6 & 1.25 & 0.231 & -13101.5 & 50430.35 \\
\hline $\begin{array}{l}\text { rockrapi - cp } \\
\text { rockrap gdd }\end{array}$ & $\begin{array}{r}40.93124 \\
\text { (dropped) }\end{array}$ & 52.68734 & 0.78 & 0.449 & -70.76093 & 152.6234 \\
\hline rockrapid $\sim i 2$ & -10129.68 & 8530.861 & -1.19 & 0.252 & -28214.3 & 7954.933 \\
\hline $\begin{array}{l}\text { rockrapid p2 } \\
\text { rockrapid d2 } \\
\text { rockrap pgdd }\end{array}$ & $\begin{array}{r}.3128427 \\
\text { (dropped) } \\
\text { (dropped) }\end{array}$ & .5048767 & 0.62 & 0.544 & -.757448 & 1.383133 \\
\hline $\begin{array}{l}\text { rockrapi icp } \\
\text { rockrap igdd }\end{array}$ & $\begin{array}{l}-58.27752 \\
\text { (dropped) }\end{array}$ & 55.03966 & -1.06 & 0.305 & -174.9564 & 58.40134 \\
\hline cons & -8357.205 & 6555.566 & -1.27 & 0.221 & -22254.38 & 5539.973 \\
\hline
\end{tabular}


Saluda, SC

Linear regression

$\begin{array}{llr}\text { Number of obs } & & 22 \\ \text { F }(6,13) & \text { 13 } & . \\ \text { Prob F } & = & \dot{0} \\ \text { R-squared } & = & 0.6606 \\ \text { Root MSE } & = & 20.537\end{array}$

\begin{tabular}{|c|c|c|c|c|c|c|}
\hline saluda_yie s & Coef. & $\begin{array}{l}\text { Robust } \mathrm{HC} 3 \\
\text { Std. Err. }\end{array}$ & $t$ & $P>|t|$ & [ $95 \%$ Conf. & Interval] \\
\hline saludasc m i & -5162.909 & 13404.89 & -0.39 & 0.706 & -34122.41 & 23796.5 \\
\hline saludācp & 1.414855 & 29.85728 & 0.05 & 0.963 & -63.08787 & 65.91758 \\
\hline saluda_ōgdd & 14.25214 & 11.96689 & 1.19 & 0.255 & -11.60075 & 40.10502 \\
\hline saluda_nd̄vi2 & 3094.49 & 8757.491 & 0.35 & 0.729 & -15824.92 & 22013.9 \\
\hline salud̄a_cp2 & -.0442941 & .2597625 & -0.17 & 0.867 & -.6054768 & .5168886 \\
\hline saluda_̄gdd2 & -.7647906 & .3428671 & -2.23 & 0.044 & -1.50551 & -.0240713 \\
\hline saluda_cpgdd & -.1892139 & .9618443 & -0.20 & 0.847 & -2.267152 & 1.888724 \\
\hline saluda_ndv $\sim$ p & 4.016267 & 41.93387 & 0.10 & 0.925 & -86.57634 & 94.60888 \\
\hline saluda_ndv $\sim$ d & (dropped) & & & & & \\
\hline _cons & 2150.967 & 5099.64 & 0.42 & 0.680 & -8866.135 & 13168.07 \\
\hline
\end{tabular}

Dublin, GA

Linear regression

$\begin{array}{llr}\text { Number of obs } & & 22 \\ \mathrm{~F}(8, \quad 12) & = \\ \text { Prob }>\text { F } & = & . \\ \text { R-squared } & = & 0.5419 \\ \text { Root MSE } & = & 22.012\end{array}$

\begin{tabular}{|c|c|c|c|c|c|c|}
\hline dublinga_y s & Coef. & $\begin{array}{c}\text { Robust } \mathrm{HC} 3 \\
\text { Std. Err. }\end{array}$ & t & $P>|t|$ & [ $95 \%$ Conf. & Interval] \\
\hline ablinga_m i & 5928.043 & 15530.68 & 0.38 & 0.709 & -27910.41 & 39766.5 \\
\hline dublin_ecp & 50.88298 & 109.3146 & 0.47 & 0.650 & -187.293 & 289.0589 \\
\hline dublin_sdd & 940.5228 & 1213.476 & 0.78 & 0.453 & -1703.415 & 3584.46 \\
\hline dublin_ndvi2 & -4358.616 & 12669.88 & -0.34 & 0.737 & -31963.91 & 23246.68 \\
\hline dublīn_cp2 & .2108548 & 1.254422 & 0.17 & 0.869 & -2.522296 & 2.944005 \\
\hline dublin_ḡdd2 & -1.122528 & 2.160638 & -0.52 & 0.613 & -5.830154 & 3.585098 \\
\hline dublin_cpgdd & 1.172193 & 2.732283 & 0.43 & 0.676 & -4.78094 & 7.125326 \\
\hline dublin_ndv $\sim p$ & -79.49889 & 189.6148 & -0.42 & 0.682 & -492.6341 & 333.6363 \\
\hline dublin_ndv $\sim$ d & -1473.105 & 1899.087 & -0.78 & 0.453 & -5610.86 & 2664.649 \\
\hline _cons & -1939.243 & 4821.581 & -0.40 & 0.695 & -12444.57 & 8566.081 \\
\hline
\end{tabular}

Booneville, MS

Linear regression

$\begin{array}{llr}\text { Number of obs } & & 20 \\ \text { F }(6, \quad 13) & 1.29 \\ \text { Prob }>\text { F } & =0.3291 \\ \text { R-squared } & =0.3436 \\ \text { Root MSE } & =13.708\end{array}$

\begin{tabular}{|c|c|c|c|c|c|c|}
\hline booneville s & Coef. & $\begin{array}{l}\text { Robust } \mathrm{HC} 3 \\
\text { Std. Err. }\end{array}$ & $t$ & $P>|t|$ & [ $95 \%$ Conf. & Interval] \\
\hline booneville i & 4152.53 & 7477.348 & 0.56 & 0.588 & -12001.3 & 20306.36 \\
\hline $\begin{array}{l}\text { boonevil _cp } \\
\text { boonevi _gdd }\end{array}$ & $\begin{array}{r}10.4921 \\
\text { (dropped) }\end{array}$ & 24.16021 & 0.43 & 0.671 & -41.70286 & 62.68706 \\
\hline boonevil̄̄ i2 & -2725.497 & 4757.843 & -0.57 & 0.577 & -13004.19 & 7553.198 \\
\hline $\begin{array}{l}\text { boonevill p2 } \\
\text { boonevill d }\end{array}$ & $\begin{array}{l}-.1605949 \\
\text { (dropped) }\end{array}$ & .1718917 & -0.93 & 0.367 & -.5319444 & .2107545 \\
\hline boonevi pgdd & -.0394621 & .1350786 & -0.29 & 0.775 & -.3312817 & .2523575 \\
\hline $\begin{array}{l}\text { boonevil icp } \\
\text { boonevi igdd }\end{array}$ & $\begin{array}{l}-5.696794 \\
\text { (dropped) }\end{array}$ & 28.86732 & -0.20 & 0.847 & -68.06084 & 56.66725 \\
\hline cons & -1555.323 & 2928.342 & -0.53 & 0.604 & -7881.621 & 4770.974 \\
\hline
\end{tabular}


Watervalley, MS

Linear regression

$\begin{array}{llr}\text { Number of obs } & & 22 \\ \text { F }(9,12) & 1.44 \\ \text { Prob }>\text { F } & = & 0.2729 \\ \text { R-squared } & = & 0.4371 \\ \text { Root MSE } & = & 19.864\end{array}$

\begin{tabular}{|c|c|c|c|c|c|c|}
\hline watervalle s & Coef. & $\begin{array}{c}\text { Robust } \mathrm{HC} 3 \\
\text { Std. Err. }\end{array}$ & $t$ & $P>|t|$ & [95\% Conf. & Interval] \\
\hline Lle $i$ & 9721 & 13227.12 & 0 & $0.4+2$ & -1909753 & 11.32 \\
\hline aterval _cp & -36.63724 & 78.36191 & -0.4 & 0.648 & -207.3732 & 134.0987 \\
\hline aterva $\sim \bar{g} d d$ & 100.7871 & 166.9041 & 0.60 & 0.557 & -262.8658 & 464.4399 \\
\hline waterval $\bar{l} \sim i 2$ & -5995.746 & 8216.099 & -0.73 & 0.480 & -23897.09 & 11905.6 \\
\hline watervall p2 & -.2863893 & .2645241 & -1.08 & 0.300 & -.8627379 & .2899592 \\
\hline watervall d2 & -.7269741 & .536239 & -1.36 & 0.200 & -1.895339 & .4413903 \\
\hline aterva pgdd & .5709007 & .58 & 0.98 & 0.346 & -.6960426 & 7844 \\
\hline aterval icp & 54.18439 & 100.8791 & 0.54 & 0.601 & -165.6123 & 273.9811 \\
\hline aterva igdd & -124.9721 & 216.9599 & -0.58 & 0.575 & -597.6871 & 347.7429 \\
\hline cons & -3905.206 & 5447.918 & -0.72 & 0.487 & -15775.2 & 7964.787 \\
\hline
\end{tabular}

Batesville, MS

Linear regression

$\begin{array}{llr}\text { Number of obs } & = & 22 \\ \mathrm{~F}(5, \quad 16) & = & 1.25 \\ \text { Prob }>\text { F } & = & 0.3333 \\ \text { R-squared } & =0.3607 \\ \text { Root MSE } & = & 19.472\end{array}$

\begin{tabular}{|c|c|c|c|c|c|c|}
\hline batesville s & Coef. & $\begin{array}{l}\text { Robust HC3 } \\
\text { Std. Err. }\end{array}$ & $t$ & $P>|t|$ & [95\% Conf. & Interval ] \\
\hline atesville i & -5560.661 & 10941.68 & -0.51 & 0.618 & -28755.98 & 17634.66 \\
\hline $\begin{array}{l}\text { batesvil _cp } \\
\text { batesvi - gdd }\end{array}$ & $\begin{array}{l}-55.12431 \\
\text { (dropped) }\end{array}$ & 69.5226 & -0.79 & 0.439 & -202.5056 & 92.25701 \\
\hline batesvili $\sim$ i2 & 3280.614 & 6920.996 & 0.47 & 0.642 & -11391.24 & 17952.47 \\
\hline oatesvill p2 & -.0291262 & .3238844 & -0.09 & 0.929 & -.7157305 & .6574782 \\
\hline atesvill d2 & (dropped) & & & & & \\
\hline patesvi pgdd & (dropped) & & & & & \\
\hline batesvil icp & 83.43634 & 90.12758 & 0.93 & 0.368 & -107.6256 & 274.4983 \\
\hline atesvi igdd & (dropped) & & & & & \\
\hline _cons & 2337.927 & 4320.948 & 0.54 & 0.596 & -6822.073 & 11497.93 \\
\hline
\end{tabular}

Yazoo City, MS

Linear regression

$\begin{array}{llr}\text { Number of obs } & 22 \\ \text { F 9, } 12) & 4.82 \\ \text { Prob }>\text { F } & =0068 \\ \text { R-squared } & =0.7479 \\ \text { Root MSE } & = & 15.487\end{array}$

\begin{tabular}{|c|c|c|c|c|c|c|}
\hline yazoocitym s & Coef. & $\begin{array}{l}\text { Robust } \mathrm{HC} 3 \\
\text { Std. Err. }\end{array}$ & $t$ & $P>|t|$ & [95\% Conf. & Interval ] \\
\hline yazoocitym i & 406.4111 & 9089.379 & 0.04 & 0.965 & -19397.64 & 20210.47 \\
\hline yazoo_cp & 6.177143 & 73.10144 & 0.08 & 0.934 & -153.0972 & 165.4515 \\
\hline yazoo_gdd & -21.94278 & 21.07611 & -1.04 & 0.318 & -67.86368 & 23.97811 \\
\hline yazoo_ndvi2 & -554.8478 & 5370.729 & -0.10 & 0.919 & -12256.66 & 11146.97 \\
\hline yazōo_cp2 & -.3237326 & .4301363 & -0.75 & 0.466 & -1.260919 & .613454 \\
\hline yazoo_ḡdd2 & .047459 & .0584679 & 0.81 & 0.433 & -.0799317 & .1748497 \\
\hline yazoo_cpgdd & .1889315 & .3285902 & 0.57 & 0.576 & -.5270051 & .9048681 \\
\hline yazoo_ndvicp & 2.625511 & 82.31787 & 0.03 & 0.975 & -176.7297 & 181.9807 \\
\hline yazoo_ndvi d & 21.45542 & 24.46848 & 0.88 & 0.398 & -31.85682 & 74.76766 \\
\hline _cons & 91.09072 & 3905.966 & 0.02 & 0.982 & -8419.277 & 8601.459 \\
\hline
\end{tabular}


Angelica, NY

Linear regression

$\begin{array}{llr}\text { Number of obs } & = & 22 \\ \text { F }(9,12) & 483.52 \\ \text { Prob }>\text { F } & =0000 \\ \text { R-squared } & =0.5440 \\ \text { Root MSE } & =11.85\end{array}$

\begin{tabular}{|c|c|c|c|c|c|c|}
\hline angelicany s & Coef. & $\begin{array}{c}\text { Robust } \mathrm{HC} 3 \\
\text { Std. Err. }\end{array}$ & $t$ & $P>|t|$ & [ $95 \%$ Conf. & Interval] \\
\hline angelicany i & -4647.59 & 3018.533 & -1.54 & 0.150 & -11224.41 & 1929.229 \\
\hline angelica_cp & 41.89273 & 38.60846 & 1.09 & 0.299 & -42.22788 & 126.0133 \\
\hline angelica_ḡdd & -4.359822 & 52.74158 & -0.08 & 0.935 & -119.2739 & 110.5542 \\
\hline angelica_n 2 & 3020.146 & 1997.72 & 1.51 & 0.156 & -1332.511 & 7372.803 \\
\hline angelica_cp2 & -.0612981 & .3847426 & -0.16 & 0.876 & -.8995802 & .7769839 \\
\hline angelica_g 2 & .0285959 & .0446938 & 0.64 & 0.534 & -.0687836 & .1259754 \\
\hline angelica_c d & -.1262876 & .2790127 & -0.45 & 0.659 & -.7342041 & .4816289 \\
\hline angelica_n p & -46.45034 & 49.12434 & -0.95 & 0.363 & -153.4831 & 60.58241 \\
\hline angelica_n d & 5.993336 & 63.25981 & 0.09 & 0.926 & -131.838 & 143.8246 \\
\hline _cons & 1844.709 & 1191.535 & 1.55 & 0.148 & -751.4232 & 4440.84 \\
\hline
\end{tabular}

Riverhead, NY

Linear regression

$\begin{array}{llr}\text { Number of obs } & & 22 \\ \mathrm{~F}(9, \quad 12) & =0.94 \\ \text { Prob }>\text { F } & = & 0.5294 \\ \text { R-squared } & = & 0.2043 \\ \text { Root MSE } & = & 17.183\end{array}$

\begin{tabular}{|c|c|c|c|c|c|c|}
\hline & & Robust $\mathrm{HC} 3$ & & & & \\
\hline riverheadn $\sim$ s & Coef. & Std. Err. & $t$ & $P>|t|$ & [95\% Conf. & Interval] \\
\hline riverheadn i & 148.6135 & 1841.549 & 0.08 & 0.937 & -3863.777 & 4161.004 \\
\hline riverhead_cp & 13.4933 & 17.22172 & 0.78 & 0.449 & -24.0296 & 51.0162 \\
\hline riverhe _ōgdd & .9892921 & 1.506402 & 0.66 & 0.524 & -2.292876 & 4.27146 \\
\hline riverhead $\sim i 2$ & -303.0554 & 1767.563 & -0.17 & 0.867 & -4154.245 & 3548.134 \\
\hline riverhead p2 & -.113694 & .1560415 & -0.73 & 0.480 & -.4536791 & .2262912 \\
\hline riverhead d2 & -.0011655 & .0020603 & -0.57 & 0.582 & -.0056544 & .0033234 \\
\hline riverhe pgdd & -.0603439 & .0566284 & -1.07 & 0.308 & -.1837266 & .0630388 \\
\hline riverhea icp & -1.569347 & 37.71838 & -0.04 & 0.967 & -83.75064 & 80.61195 \\
\hline riverhe igdd & .5115184 & 2.064293 & 0.25 & 0.808 & -3.98619 & 5.009227 \\
\hline _cons & -88.92248 & 470.3161 & -0.19 & 0.853 & -1113.653 & 935.8083 \\
\hline
\end{tabular}

Fredonia, NY

Linear regression

$\begin{array}{llr}\text { Number of obs } & & 22 \\ \text { F }(9, \quad 12) & 0.40 \\ \text { Prob }>\text { F } & =0113 \\ \text { R-squared } & =0.2610 \\ \text { Root MSE } & = & 11.162\end{array}$

\begin{tabular}{|c|c|c|c|c|c|c|}
\hline fredoniany s & Coef. & $\begin{array}{l}\text { Robust HC3 } \\
\text { Std. Err. }\end{array}$ & $t$ & $P>|t|$ & {$[95 \%$} & Interval] \\
\hline fredoniany i & 203.144 & 307.9157 & 0.66 & 0.522 & -467.7468 & 874.0347 \\
\hline fredonia_cp & -.0514637 & 8.877371 & -0.01 & 0.995 & -19.39359 & 19.29067 \\
\hline fredonia gdd & -.3535607 & 1.059763 & -0.33 & 0.744 & -2.662587 & 1.955465 \\
\hline fredonia_n 2 & -117.1984 & 272.1489 & -0.43 & 0.674 & -710.1599 & 475.7632 \\
\hline fredonia_cp2 & .044514 & .1547623 & 0.29 & 0.779 & -.2926842 & .3817121 \\
\hline fredonia_g 2 & .0007652 & .0031563 & 0.24 & 0.813 & -.0061118 & .0076422 \\
\hline fredonia_c d & .0327744 & .0540759 & 0.61 & 0.556 & -.0850469 & .1505956 \\
\hline fredonia_n p & -5.757881 & 8.394044 & -0.69 & 0.506 & -24.04693 & 12.53117 \\
\hline $\begin{array}{r}\text { fredonia_n d } \\
\text { cons }\end{array}$ & $\begin{array}{r}-.3029045 \\
61.40246\end{array}$ & $\begin{array}{l}1.165247 \\
134.1585\end{array}$ & $\begin{array}{r}-0.26 \\
0.46\end{array}$ & $\begin{array}{l}0.799 \\
0.655\end{array}$ & $\begin{array}{r}-2.84176 \\
-230.9037\end{array}$ & $\begin{array}{l}2.235951 \\
353.7086\end{array}$ \\
\hline
\end{tabular}


Du Quoin, IL

Linear regression

$\begin{array}{llr}\text { Number of obs } & & 22 \\ \text { F }(9,12) & 0.40 \\ \text { Prob }>\text { F } & = & 0.9098 \\ \text { R-squared } & =0.6282 \\ \text { Root MSE } & = & 16.295\end{array}$

\begin{tabular}{|c|c|c|c|c|c|c|}
\hline duquoinil_ s & Coef. & $\begin{array}{l}\text { Robust HC3 } \\
\text { Std. Err. }\end{array}$ & $t$ & $P>|t|$ & [ $95 \%$ Conf. & Interval] \\
\hline duquoinil_ i & -2174.452 & 3561.786 & -0.61 & 0.553 & -9934.917 & 5586.013 \\
\hline duquoin_cp & -69.10853 & 66.07885 & -1.05 & 0.316 & -213.082 & 74.86492 \\
\hline duquoin_ḡdd & -11.17822 & 22.90449 & -0.49 & 0.634 & -61.08283 & 38.72638 \\
\hline duquoin nd 2 & 707.1143 & 1813.931 & 0.39 & 0.703 & -3245.102 & 4659.33 \\
\hline duquoiñ_cp2 & .3351413 & .2946221 & 1.14 & 0.278 & -.3067851 & .9770677 \\
\hline duquoin_gdd2 & .0019928 & .0249811 & 0.08 & 0.938 & -.0524363 & .0564219 \\
\hline duquoin_cp d & .0322938 & .1217361 & 0.27 & 0.795 & -.2329465 & .2975341 \\
\hline duquoin_nd p & 82.61601 & 79.90391 & 1.03 & 0.322 & -91.47964 & 256.7117 \\
\hline duquoin_nd d & 14.63697 & 29.59524 & 0.49 & 0.630 & -49.84553 & 79.11946 \\
\hline _cons & 1343.449 & 1767.785 & 0.76 & 0.462 & -2508.223 & 5195.122 \\
\hline
\end{tabular}

Minonk, IL

Linear regression

$\begin{array}{llr}\text { Number of obs } & & 22 \\ \text { F }(9, \quad 12) & 2.27 \\ \text { Prob }>\text { F } & =0930 \\ \text { R-squared } & =0.8160 \\ \text { Root MSE } & =17.274\end{array}$

\begin{tabular}{|c|c|c|c|c|c|c|}
\hline minonkil_y s & Coef. & $\begin{array}{c}\text { Robust HC3 } \\
\text { Std. Err. }\end{array}$ & $t$ & $P>|t|$ & [ $95 \%$ Conf. & Interval] \\
\hline minonkil_m i & 8618.975 & 12483.57 & 0.69 & 0.503 & -18580.39 & 35818.34 \\
\hline minon $\bar{k} \_c p$ & -47.37575 & 133.5898 & -0.35 & 0.729 & -338.443 & 243.6915 \\
\hline minonk_ogdd & -57.58734 & 295935.3 & -0.00 & 1.000 & -644845.2 & 644730 \\
\hline minonk_ndvi2 & -5738.639 & 8592.355 & -0.67 & 0.517 & -24459.77 & 12982.49 \\
\hline minonk_cp2 & -.9986846 & .5794672 & -1.72 & 0.110 & -2.261235 & .2638661 \\
\hline minonk_ḡdd2 & -.5143097 & 6112.681 & -0.00 & 1.000 & -13318.9 & 13317.87 \\
\hline minonk_cpgdd & .6191451 & 2801.624 & 0.00 & 1.000 & -6103.595 & 6104.833 \\
\hline minonk_ndv $\sim$ p & 88.98763 & 167.4952 & 0.53 & 0.605 & -275.9531 & 453.9283 \\
\hline minonk_ndv $\sim \mathrm{d}$ & 65.08113 & 358447.7 & 0.00 & 1.000 & -780925.4 & 781055.6 \\
\hline cons & -3224.071 & 4446.585 & -0.73 & 0.482 & -12912.35 & 6464.205 \\
\hline
\end{tabular}

Ardmore, SD

Linear regression

$\begin{array}{llr}\text { Number of obs } & & 22 \\ \text { F }(9,12) & 4.71 \\ \text { Prob }>\text { F } & = & 0.0075 \\ \text { R-squared } & = & 0.7006 \\ \text { Root MSE } & = & 11.872\end{array}$

\begin{tabular}{|c|c|c|c|c|c|c|}
\hline ardmoresd_ s & Coef. & $\begin{array}{c}\text { Robust } \mathrm{HC} 3 \\
\text { Std. Err. }\end{array}$ & $t$ & $P>|t|$ & [95\% Conf. & Interval] \\
\hline ardmoresd_ i & 603.0854 & 1018.867 & 0.59 & 0.565 & -1616.835 & 2823.006 \\
\hline ardmore_cp & 24.73 & 27.42167 & 0.90 & 0.385 & -35.01668 & 84.47669 \\
\hline ardmore_gdd & -2.312138 & 2.337933 & -0.99 & 0.342 & -7.406056 & 2.781781 \\
\hline ardmore $\overline{n d} \sim 2$ & -452.4939 & 777.2393 & -0.58 & 0.571 & -2145.953 & 1240.965 \\
\hline ardmorēe_cp2 & -.4442978 & .5012455 & -0.89 & 0.393 & -1.536418 & .6478223 \\
\hline ardmore_ḡdd2 & .0048511 & .0040597 & 1.19 & 0.255 & -.0039942 & .0136963 \\
\hline ardmore_cp d & .0232084 & .083084 & 0.28 & 0.785 & -.157816 & .2042328 \\
\hline ardmore_nd $\sim p$ & -27.90225 & 27.70333 & -1.01 & 0.334 & -88.26261 & 32.45811 \\
\hline ardmore_nd d & 2.556933 & 2.616229 & 0.98 & 0.348 & -3.143339 & 8.257206 \\
\hline cons & -109.2068 & 401.5431 & -0.27 & 0.790 & -984.094 & 765.6804 \\
\hline
\end{tabular}


Windsor, IL

Linear regression

$\begin{array}{llr}\text { Number of obs } & & 22 \\ \text { F }(9, \quad 12) & 3.11 \\ \text { Prob }>\text { F } & = & 0.0354 \\ \text { R-squared } & = & 0.7346 \\ \text { Root MSE } & = & 16.221\end{array}$

\begin{tabular}{|c|c|c|c|c|c|c|}
\hline windsoril_ s & Coef. & $\begin{array}{c}\text { Robust } \mathrm{HC} 3 \\
\text { Std. Err. }\end{array}$ & $t$ & $P>|t|$ & [ $95 \%$ Conf. & Interval] \\
\hline windsoril_ i & 2518.301 & 2868.266 & 0.88 & 0.397 & -3731.114 & 8767.715 \\
\hline windsor $\mathrm{cp}$ & 9.522434 & 48.91393 & 0.19 & 0.849 & -97.05187 & 116.0967 \\
\hline windsor_ḡdd & -.5731833 & .9884681 & -0.58 & 0.573 & -2.72687 & 1.580504 \\
\hline windsor nd 2 & -1328.247 & 2101.81 & -0.63 & 0.539 & -5907.697 & 3251.202 \\
\hline windsor $\mathrm{cp} 2$ & .3865585 & .6925499 & 0.56 & 0.587 & -1.122378 & 1.895495 \\
\hline windsor ḡdd2 & .0001308 & .000685 & 0.19 & 0.852 & -.0013617 & .0016232 \\
\hline windsor $\mathrm{cp} \sim \mathrm{d}$ & -.0127345 & .0291876 & -0.44 & 0.670 & -.0763288 & .0508597 \\
\hline windsor nd p & -22.41855 & 60.28347 & -0.37 & 0.716 & -153.7649 & 108.9278 \\
\hline windsor ${ }^{-}$nd d & .6550718 & 1.121387 & 0.58 & 0.570 & -1.78822 & 3.098363 \\
\hline cons & -949.006 & 941.9511 & -1.01 & 0.334 & -3001.341 & 1103.329 \\
\hline
\end{tabular}

White Hall, IL

Linear regression

$\begin{array}{llr}\text { Number of obs } & & 22 \\ \text { F } 7, \quad 13) & = & . \\ \text { Prob }>\text { F } & = & . \\ \text { R-squared } & = & 0.6901 \\ \text { Root MSE } & = & 15.926\end{array}$

\begin{tabular}{|c|c|c|c|c|c|c|}
\hline whitehalli s & Coef. & $\begin{array}{l}\text { Robust HC3 } \\
\text { Std. Err. }\end{array}$ & $\mathrm{t}$ & $P>|t|$ & [95\% Conf. & Inte \\
\hline whitehalli i & 5119.446 & 2377.349 & 2.15 & 0.051 & -16.50501 & 10255.4 \\
\hline vhitehall_cp & -42.98011 & 26.71576 & -1.61 & 0.132 & -100.696 & 14.73578 \\
\hline whiteha _ōgdd & -77.73002 & 84.18356 & -0.92 & 0.373 & -259.5975 & 104.1375 \\
\hline whitehali $\sim$ i2 & -3757.07 & 1774.348 & $-2 \cdot 12$ & 0.054 & -7590.315 & 76.17475 \\
\hline whitehall p2 & -.4055809 & .240269 & -1.69 & 0.115 & -.9246504 & .1134887 \\
\hline whitehall d2 & .0516524 & .0561728 & 0.92 & 0.375 & -.0697016 & .1730064 \\
\hline whiteha pgdd & 4.52806 & 4.904035 & 0.92 & 0.373 & -6.066464 & 15.12258 \\
\hline whitehal icp & 66.74442 & 36.70116 & 1.82 & 0.092 & -12.54362 & 146.0325 \\
\hline hiteha igdd & (dropped) & & & & & \\
\hline _cons & -1609.752 & 782.9557 & -2.06 & 0.060 & -3301.225 & 81.72059 \\
\hline
\end{tabular}

\section{Beaverdam, KY}

Linear regression

$\begin{array}{llr}\text { Number of obs } & & 22 \\ \text { F }(9, \quad 12) & 0.70 \\ \text { Prob }>\text { F } & =0.6971 \\ \text { R-squared } & = & 0.5033 \\ \text { Root MSE } & = & 20.473\end{array}$

\begin{tabular}{|c|c|c|c|c|c|c|}
\hline beaverdamk s & Coef. & $\begin{array}{l}\text { Robust HC3 } \\
\text { Std. Err. }\end{array}$ & t & $P>|t|$ & [95\% Conf. & Interval ] \\
\hline beaverdamk i & 4264.86 & 27806.49 & 0.15 & 0.881 & -56320.28 & 64849.99 \\
\hline beaverdam_cp & 51.54705 & 238.2483 & 0.22 & 0.832 & -467.5513 & 570.6454 \\
\hline beaverd _gdd & .1425247 & 7.953291 & 0.02 & 0.986 & -17.18621 & 17.47126 \\
\hline beaverdam i2 & -2064.914 & 19493.76 & -0.11 & 0.917 & -44538.16 & 40408.34 \\
\hline beaverdam p2 & -.3004933 & 1.260657 & -0.24 & 0.816 & -3.047228 & 2.446241 \\
\hline beaverdam d2 & -.0005008 & .0021397 & -0.23 & 0.819 & -.0051629 & .0041612 \\
\hline beaverd pgdd & .0243874 & .1253571 & 0.19 & 0.849 & -.2487423 & .297517 \\
\hline beaverda icp & -69.44028 & 252.2512 & -0.28 & 0.788 & -619.0485 & 480.168 \\
\hline beaverd igdd & -.351043 & 8.530373 & -0.04 & 0.968 & -18.93713 & 18.23504 \\
\hline _cons & -1860.97 & 9869.187 & -0.19 & 0.854 & -23364.08 & 19642.14 \\
\hline
\end{tabular}


Providence, KY

Linear regression

$\begin{array}{lrr}\text { Number of obs } & & 22 \\ \text { F }(9, \quad 12) & =3.63 \\ \text { Prob }>\text { F } & =0206 \\ \text { R-squared } & =0.6771 \\ \text { Root MSE } & = & 16.521\end{array}$

\begin{tabular}{|c|c|c|c|c|c|c|}
\hline providence s & Coef. & $\begin{array}{c}\text { Robust } \mathrm{HC} 3 \\
\text { Std. Err. }\end{array}$ & $t$ & $P>|t|$ & [95\% Conf. & Interval] \\
\hline covidence i & -2748.798 & 17131.45 & -0.16 & 0.875 & -40075.03 & 34577.43 \\
\hline covidence $\sim p$ & 121.3655 & 143.1333 & 0.85 & 0.413 & -190.4952 & 433.2262 \\
\hline rovidn $\sim$ gdd & -.9209739 & 2.173371 & -0.42 & 0.679 & -5.656343 & 3.814395 \\
\hline rovidnec $\sim$ i2 & 2986.441 & 11726.35 & 0.25 & 0.803 & -22563.09 & 28535.97 \\
\hline covidnec $\sim \mathrm{p} 2$ & .0580032 & .5179406 & 0.11 & 0.913 & -1.070492 & 1.186499 \\
\hline covidnec $\sim \mathrm{d} 2$ & -.0000187 & .0001252 & -0.15 & 0.884 & -.0002915 & .0002541 \\
\hline ovidn pgdd & -.0052473 & .0112088 & -0.47 & 0.648 & -.0296691 & .0191746 \\
\hline covidnece $\sim p$ & -159.3928 & 201.817 & -0.79 & 0.445 & -599.1143 & 280.3287 \\
\hline covidn igdd & 1.310975 & 2.869223 & 0.46 & 0.656 & -4.940525 & 7.562475 \\
\hline cons & 470.4625 & 6518.562 & 0.07 & 0.944 & -13732.26 & 14673.19 \\
\hline
\end{tabular}

Farmville, VA

Linear regression

$\begin{array}{llr}\text { Number of obs } & = & 22 \\ \mathrm{~F}(9, \quad 12) & = & 1.43 \\ \text { Prob }>\mathrm{F} & = & 0.2747 \\ \text { R-squared } & = & 0.5080 \\ \text { Root MSE } & = & 28.946\end{array}$

\begin{tabular}{|c|c|c|c|c|c|c|}
\hline farmvillev s & Coef. & $\begin{array}{l}\text { Robust } \mathrm{HC} 3 \\
\text { Std. Err. }\end{array}$ & $t$ & $P>|t|$ & [95\% Conf. & Interval] \\
\hline & 17001 & & 0 & & & \\
\hline farmvillev i & 11081.47 & 24554.36 & 0.45 & 0.660 & -42417.88 & 64580.81 \\
\hline farmville_cp & -320.0663 & 216.1174 & -1.48 & 0.164 & -790.9456 & 150.813 \\
\hline farmvil _ gdd & -4.270826 & 3.905717 & -1.09 & 0.296 & -12.78065 & 4.238999 \\
\hline farmville $-i 2$ & -10796.19 & 16836.24 & -0.64 & 0.533 & -47479.2 & 25886.83 \\
\hline farmville p2 & .0726185 & .8179263 & 0.09 & 0.931 & -1.70949 & 1.854727 \\
\hline farmville d2 & -.001253 & .0013446 & -0.93 & 0.370 & -.0041827 & .0016768 \\
\hline farmvil pgdd & -.0467279 & .0602808 & -0.78 & 0.453 & -.1780686 & .0846127 \\
\hline farmvill icp & 404.2374 & 266.5321 & 1.52 & 0.155 & -176.4861 & 984.9609 \\
\hline farmvil igdd & 6.563443 & 5.59653 & 1.17 & 0.264 & -5.630349 & 18.75723 \\
\hline _cons & -1947.377 & 9089.506 & -0.21 & 0.834 & -21751.71 & 17856.96 \\
\hline
\end{tabular}

\section{Heppner, OR}

Linear regression

$\begin{array}{lrr}\text { Number of obs } & & 22 \\ \text { F }(9, \quad 12) & 0.20 \\ \text { Prob }>\text { F } & =0990 \\ \text { R-squared } & =0.2178 \\ \text { Root MSE } & = & 27.669\end{array}$

\begin{tabular}{|c|c|c|c|c|c|c|}
\hline heppneror_ s & Coef. & $\begin{array}{l}\text { Robust } \mathrm{HC} 3 \\
\text { Std. Err. }\end{array}$ & $t$ & $P>|t|$ & [ $95 \%$ Conf. & Interval] \\
\hline heppneror $\sim i$ & -1254.366 & 4258.113 & -0.29 & 0.773 & -10532 & 8023.266 \\
\hline heppner_cp & 2.376535 & 200.7005 & 0.01 & 0.991 & -434.9123 & 439.6654 \\
\hline heppner gdd & 1.41442 & 24.66084 & 0.06 & 0.955 & -52.31693 & 55.14577 \\
\hline heppner_nd 2 & 1817.604 & 5590.579 & 0.33 & 0.751 & -10363.22 & 13998.43 \\
\hline heppners_cp2 & 4.171648 & 12.03211 & 0.35 & 0.735 & -22.04406 & 30.38735 \\
\hline heppner_gdd2 & -.0068911 & .1111649 & -0.06 & 0.952 & -.2490986 & .2353164 \\
\hline heppner_cp d & .2322885 & 1.461083 & 0.16 & 0.876 & -2.951138 & 3.415715 \\
\hline heppner_nd p & -98.74451 & 352.8957 & -0.28 & 0.784 & -867.6382 & 670.1491 \\
\hline $\begin{array}{r}\text { heppner_nd d } \\
\text { cons }\end{array}$ & $\begin{array}{r}-6.064508 \\
476.6394\end{array}$ & $\begin{array}{r}52.17412 \\
890.027\end{array}$ & $\begin{array}{r}-0.12 \\
0.54\end{array}$ & $\begin{array}{l}0.909 \\
0.602\end{array}$ & $\begin{array}{l}-119.7422 \\
-1462.563\end{array}$ & $\begin{array}{l}107.6131 \\
2415.842\end{array}$ \\
\hline
\end{tabular}


Eltopia, WA

Linear regression

$\begin{array}{llr}\text { Number of obs } & & 21 \\ \text { F }(9, \quad 11) & 1.28 \\ \text { Prob }>\text { F } & =0.3455 \\ \text { R-squared } & =0.6910 \\ \text { Root MSE } & =7.7538\end{array}$

\begin{tabular}{|c|c|c|c|c|c|c|}
\hline eltopiawa_ s & Coef. & $\begin{array}{c}\text { Robust } \mathrm{HC} 3 \\
\text { Std. Err. }\end{array}$ & $t$ & $P>|t|$ & [95\% Conf. & Interval] \\
\hline Itopiawa_ i & 2506.975 & 5218.334 & 0.48 & 0.640 & -8978.5 & 13992.45 \\
\hline eltopia_cp & -281.7797 & 289.4438 & -0.97 & 0.351 & -918.8413 & 355.2819 \\
\hline eltopia $\bar{g} d d$ & 1.029907 & 3.371223 & 0.31 & 0.766 & -6.390104 & 8.449919 \\
\hline eltopia_nnd 2 & -2290.043 & 4002.23 & -0.57 & 0.579 & -11098.89 & 6518.806 \\
\hline eltopiā_cp2 & -12.19647 & 11.03337 & -1.11 & 0.293 & -36.48075 & 12.08781 \\
\hline eltopia gdd2 & .0011412 & .0031933 & 0.36 & 0.728 & -.0058871 & .0081695 \\
\hline eltopia cp d & .0109702 & .1925721 & 0.06 & 0.956 & -.4128782 & .4348186 \\
\hline eltopia_nd p & 471.4662 & 450.5588 & 1.05 & 0.318 & -520.2071 & 1463.14 \\
\hline eltopia_nd d & -1.621549 & 4.935082 & -0.33 & 0.749 & -12.48359 & 9.240495 \\
\hline cons & -506.5165 & 1716.172 & -0.30 & 0.773 & -4283.785 & 3270.752 \\
\hline
\end{tabular}

Menomonie, WI

Linear regression

$\begin{array}{llr}\text { Number of obs } & & 22 \\ \mathrm{~F}(9, \quad 12) & =2.90 \\ \text { Prob }>\text { F } & = & 0.0445 \\ \text { R-squared } & =0.6067 \\ \text { Root MSE } & = & 15.984\end{array}$

\begin{tabular}{|c|c|c|c|c|c|c|}
\hline menomoniew s & Coef. & $\begin{array}{l}\text { Robust } \mathrm{HC} 3 \\
\text { Std. Err. }\end{array}$ & $t$ & $P>|t|$ & [ $95 \%$ Conf. & Interval] \\
\hline enomoniew $\sim$ & 9357.289 & 13582.73 & 0.69 & 0.504 & -20236.93 & 38951.51 \\
\hline enomonie_cp & -7.212612 & 157.999 & -0.05 & 0.964 & -351.4629 & 337.0377 \\
\hline menomon $\sim \bar{g}$ dd & -1.734742 & 4.536782 & -0.38 & 0.709 & -11.61954 & 8.150057 \\
\hline menomoniēi2 & -6221.92 & 9096.592 & -0.68 & 0.507 & -26041.69 & 13597.85 \\
\hline onie p2 & .0254062 & .9802063 & 0.03 & 0.980 & -2.11028 & 2.161092 \\
\hline enomonie d2 & .0005185 & .0008857 & 0.59 & 0.569 & -.0014113 & .0024484 \\
\hline menomon $\sim$ pgdd & .0550166 & .0628429 & 0.88 & 0.399 & -.0819063 & .1919395 \\
\hline menomoni icp & 1.639145 & 172.359 & 0.01 & 0.993 & -373.8988 & 377.177 \\
\hline enomon igdd & 1.082636 & 5.920179 & 0 . & 0.858 & -11.81633 & 13.9816 \\
\hline _cons & -3311.216 & 5124.187 & -0.65 & 0.530 & -14475.86 & 7853.429 \\
\hline
\end{tabular}

Arlington, WI

Linear regression

$\begin{array}{llr}\text { Number of obs } & & 22 \\ \mathrm{~F}(9, \quad 12) & = & 1.35 \\ \text { Prob }>\text { F } & = & 0.3095 \\ \text { R-squared } & = & 0.6555 \\ \text { Root MSE } & = & 15.59\end{array}$

\begin{tabular}{|c|c|c|c|c|c|c|}
\hline arlingtonw $\sim \mathrm{s}$ & Coef. & $\begin{array}{l}\text { Robust } \mathrm{HC} 3 \\
\text { Std. Err. }\end{array}$ & t & $P>|t|$ & [ $95 \%$ Conf. & rval] \\
\hline arling & 2408.849 & 6390.878 & 0.38 & 0.713 & -11515.68 & 16333.38 \\
\hline arlington_cp & 12.05749 & 136.8279 & 0.09 & 0.931 & -286.0649 & 310.1799 \\
\hline arlingt _ g dd & 1.000102 & 3.869617 & 0.26 & 0.800 & -7.431069 & 9.431273 \\
\hline arlington $\sim$ i2 & -1491.056 & 4464.927 & -0.33 & 0.744 & -11219.3 & 8237.184 \\
\hline arlington $\sim \mathrm{p} 2$ & -.365963 & .9467423 & -0.39 & 0.706 & -2.428737 & 1.696811 \\
\hline arlington $\sim d 2$ & -.0000782 & .0019369 & -0.04 & 0.968 & -.0042982 & .0041419 \\
\hline arlingt pgdd & -.0035246 & 383 & -0.03 & 0.974 & -.2328191 & .2257698 \\
\hline to icp & -.5320992 & 84 & -0.00 & 0.997 & .2243 & 1601 \\
\hline arlingt igdd & -1.409296 & 3.896153 & -0.36 & 0.724 & -9.898283 & 7.079692 \\
\hline cons & -919.0616 & 2524.388 & -0.36 & 0.722 & -6419.231 & 4581.107 \\
\hline
\end{tabular}




\title{
Sellingsgrove, PA
}

\author{
Linear regression
}

$\begin{array}{llr}\text { Number of obs } & & 22 \\ \text { F }(5,16) & 2.27 \\ \text { Prob }>\text { F } & = & 0.0970 \\ \text { R-squared } & =0.6052 \\ \text { Root MSE } & = & 16.564\end{array}$

\begin{tabular}{|c|c|c|c|c|c|c|}
\hline sellingsgr s & Coef. & $\begin{array}{l}\text { Robust HC3 } \\
\text { Std. Err. }\end{array}$ & $t$ & $P>|t|$ & {$\left[\begin{array}{l}95 \% \\
\text { Conf. }\end{array}\right.$} & Interval] \\
\hline elingsgro i & 7770.009 & 18983.94 & 0.41 & 0.688 & -32474.14 & 48014.16 \\
\hline $\begin{array}{l}\text { selingsgro p } \\
\text { selling _gdd }\end{array}$ & $\begin{array}{r}15.83092 \\
\text { (dropped) }\end{array}$ & 80.39748 & 0.20 & 0.846 & -154.6041 & 186.266 \\
\hline sellingsḡ i2 & -5061.72 & 12010.67 & -0.42 & 0.679 & -30523.2 & 20399.76 \\
\hline $\begin{array}{l}\text { sellingsg p2 } \\
\text { sellingsg d } 2\end{array}$ & $\begin{array}{l}-.7698302 \\
\text { (dropped) }\end{array}$ & 353 & -1.73 & 0.102 & -1.710507 & .1708467 \\
\hline $\begin{array}{l}\text { selling pgdd } \\
\text { sellingsgr p } \\
\text { selling igdd }\end{array}$ & $\begin{array}{r}\text { (dropped) } \\
12.7943 \\
\text { (dropped) }\end{array}$ & 104.6399 & 0.12 & 0.904 & -209.0324 & 234.621 \\
\hline _cons & -3085.179 & 7584.319 & -0.41 & 0.690 & -19163.22 & 12992.86 \\
\hline
\end{tabular}

\section{Montrose, PA}

Linear regression

$\begin{array}{llr}\text { Number of obs } & =22 \\ \text { F } 6, \quad 15) & 18.20 \\ \text { Prob }>\text { F } & =0.0000 \\ \text { R-squared } & =0.4977 \\ \text { Root MSE } & =12.193\end{array}$

\begin{tabular}{|c|c|c|c|c|c|c|}
\hline montrosepa s & Coef. & $\begin{array}{c}\text { Robust HC } 3 \\
\text { Std. Err. }\end{array}$ & $t$ & $P>|t|$ & [ $95 \%$ Conf. & Interval] \\
\hline $\begin{array}{l}\text { montrosepa i } \\
\text { montrose_cp } \\
\text { montrose_gdd }\end{array}$ & $\begin{array}{r}-4715.934 \\
-6.24172 \\
\text { (dropped) }\end{array}$ & $\begin{array}{r}3702.487 \\
27.2698\end{array}$ & $\begin{array}{l}-1.27 \\
-0.23\end{array}$ & $\begin{array}{l}0.222 \\
0.822\end{array}$ & $\begin{array}{r}-12607.6 \\
-64.36591\end{array}$ & $\begin{array}{l}3175.729 \\
51.88247\end{array}$ \\
\hline montrose_n 2 & 2576.101 & 2012.443 & 1.28 & 0.220 & -1713.32 & 6865.522 \\
\hline $\begin{array}{l}\text { montrose_cp2 } \\
\text { montrose_g 2 }\end{array}$ & $\begin{array}{l}-.1568907 \\
\text { (dropped) }\end{array}$ & .235776 & -0.67 & 0.516 & -.6594354 & .345654 \\
\hline montrose_c $\sim \mathrm{d}$ & -.3574746 & .0875493 & -4.08 & 0.001 & -.5440816 & -.1708676 \\
\hline $\begin{array}{l}\text { montrose_n p } \\
\text { montrose_n } \sim d\end{array}$ & $\begin{array}{r}14.26023 \\
\text { (dropped) }\end{array}$ & 25.26331 & 0.56 & 0.581 & -39.58724 & 68.10771 \\
\hline $\bar{c}$ cons & 2189.699 & 1722.097 & 1.27 & 0.223 & -1480.865 & 5860.263 \\
\hline
\end{tabular}


Regression Results for Soy Yields

\section{Algona, IA}

Linear regression

$\begin{array}{llr}\text { Number of obs } & & 22 \\ \text { F }(9, \quad 12) & 0.93 \\ \text { Prob }>\text { F } & = & 0.5363 \\ \text { R-squared } & = & 0.6578 \\ \text { Root MSE } & = & 4.6957\end{array}$

\begin{tabular}{|c|c|c|c|c|c|c|}
\hline algonaia_y s & Coef. & $\begin{array}{c}\text { Robust } \mathrm{HC} 3 \\
\text { Std. Err. }\end{array}$ & $t$ & $P>|t|$ & {$[95 \%$} & Interval] \\
\hline algonaia $\mathrm{m} \sim \mathrm{i}$ & -1814.511 & 7047.873 & -0.26 & 0.801 & -17170.51 & 13541.49 \\
\hline $\begin{array}{l}\text { algonā_cp } \\
\text { alqona } \bar{q} d d\end{array}$ & $\begin{array}{l}-.7250928 \\
-.9320641\end{array}$ & $\begin{array}{l}41.22457 \\
1.537436\end{array}$ & $\begin{array}{l}-0.02 \\
-0.61\end{array}$ & $\begin{array}{l}0.986 \\
0.556\end{array}$ & $\begin{array}{r}-90.54572 \\
-4.28185\end{array}$ & $\begin{array}{l}89.09553 \\
2.417722\end{array}$ \\
\hline longona_m 2 & 1092.608 & 4268.33 & 0.26 & 0.802 & -8207.284 & 10392.5 \\
\hline algona_cp2 & -.0918152 & .1209298 & -0.76 & 0.462 & -.3552986 & .1716682 \\
\hline algona_gdd2 & .0003237 & .0006306 & 0.51 & 0.617 & -.0010502 & .0016977 \\
\hline lgona cpgdd & .0040251 & .0164963 & 0.24 & 0.811 & -.0319171 & .0399674 \\
\hline andv $\sim p$ & 3.935387 & 49.0 & 0.08 & 0.937 & -102.9148 & 110.7855 \\
\hline lgona_ndv d & .9781256 & 1.486274 & 0.66 & 0.523 & -2.260187 & 4.216438 \\
\hline cons & 778.6642 & 2941.46 & 0.26 & 0.796 & -5630.227 & 7187.556 \\
\hline
\end{tabular}

\section{Rockrapids, IA}

Linear regression

$\begin{array}{llr}\text { Number of obs } & & 22 \\ \text { F } 5, \quad 16) & 6.02 \\ \text { Prob }>\text { F } & = & 0.0026 \\ \text { R-squared } & =0.6862 \\ \text { Root MSE } & =4.2614\end{array}$

\begin{tabular}{|c|c|c|c|c|c|c|}
\hline rockrapids s & Coef. & $\begin{array}{l}\text { Robust } \mathrm{HC} 3 \\
\text { Std. Err. }\end{array}$ & t & $P>|t|$ & [ $95 \%$ Conf. & Interval] \\
\hline ockrapids i & 1058.166 & 4574.038 & 0.23 & 0.820 & -8638.361 & 10754.69 \\
\hline $\begin{array}{l}\text { rockrapi _cp } \\
\text { rockrap gdd }\end{array}$ & $\begin{array}{r}9.33645 \\
\text { (dropped) }\end{array}$ & 10.57743 & 0.88 & 0.390 & -13.08669 & 31.75959 \\
\hline cockrapid $\sim i 2$ & -459.4974 & 2618.063 & -0.18 & 0.863 & -6009.544 & 5090.549 \\
\hline $\begin{array}{l}\text { rockrapid p2 } \\
\text { rockrapid d2 } \\
\text { rockrap pgdd }\end{array}$ & $\begin{array}{l}-.0414785 \\
\text { (dropped) } \\
\text { (dropped) }\end{array}$ & .1268377 & -0.33 & 0.748 & -.3103625 & .2274054 \\
\hline $\begin{array}{l}\text { rockrapi icp } \\
\text { rockrap igdd }\end{array}$ & $\begin{array}{l}-9.931236 \\
\text { (dropped) }\end{array}$ & 11.95325 & -0.83 & 0.418 & -35.27099 & 15.40852 \\
\hline cons & -529.3868 & 1984.105 & -0.27 & 0.793 & -4735.502 & 3676.729 \\
\hline
\end{tabular}


Saluda, SC

Linear regression

$\begin{array}{llr}\text { Number of obs } & & 21 \\ \text { F }(6, \quad 12) & . \\ \text { Prob }>\text { F } & = & \dot{ } \\ \text { R-squared } & = & 0.7826 \\ \text { Root MSE } & = & 2.3972\end{array}$

\begin{tabular}{|c|c|c|c|c|c|c|}
\hline saluda_yie s & Coef. & $\begin{array}{l}\text { Robust } \mathrm{HC} 3 \\
\text { Std. Err. }\end{array}$ & $t$ & $P>|t|$ & [ $95 \%$ Conf. & Inte \\
\hline saludasc_m i & -10648.65 & 2553.056 & -4.17 & 0.001 & -16211.28 & -5086.022 \\
\hline saludācp & -31.69274 & 10.51508 & -3.01 & 0.011 & -54.60312 & -8.782355 \\
\hline saluda_ḡdd & 4.832407 & 1.600502 & 3.02 & 0.011 & 1.345213 & 8.319602 \\
\hline saluda ndvi2 & 6576.267 & 1568.007 & 4.19 & 0.001 & 3159.873 & 9992.661 \\
\hline saluda_a_cp2 & .0368131 & .0526719 & 0.70 & 0.498 & -.0779492 & .1515754 \\
\hline saluda_̄gdd2 & -.1174383 & .0449632 & -2.61 & 0.023 & -.2154047 & -.019472 \\
\hline saluda_cpgdd & -.1642622 & .1284485 & -1.28 & 0.225 & -.4441275 & .115603 \\
\hline saluda_ndv $\sim$ p & 40.50446 & 12.16288 & 3.33 & 0.006 & 14.00383 & 67.00509 \\
\hline saluda_ndv $\sim$ d & (dropped) & & & & & \\
\hline _cons & 4318.534 & 1041.499 & 4.15 & 0.001 & 2049.302 & 6587.766 \\
\hline
\end{tabular}

Dublin, GA

Linear regression

$\begin{array}{llr}\text { Number of obs } & & 22 \\ \text { F }(9,12) & 57.37 \\ \text { Prob }>\text { F } & =0000 \\ \text { R-squared } & =0.6722 \\ \text { Root MSE } & =5.0979\end{array}$

\begin{tabular}{|c|c|c|c|c|c|c|}
\hline dublinga_y s & Coef. & $\begin{array}{c}\text { Robust HC3 } \\
\text { Std. Err. }\end{array}$ & t & $P>|t|$ & {$\left[\begin{array}{lll}95 \% & \text { Conf. }\end{array}\right.$} & Interval] \\
\hline dublinga_m i & 6816.666 & 5491.27 & 1.24 & 0.238 & -5147.783 & 18781.11 \\
\hline dublin̄_cp & 31.318 & 23.70952 & 1.32 & 0.211 & -20.3406 & 82.97659 \\
\hline dublin_ggdd & 490.4518 & 248.7946 & 1.97 & 0.072 & -51.62516 & 1032.529 \\
\hline dublin_ndvi2 & -4799.059 & 4175.444 & -1.15 & 0.273 & -13896.57 & 4298.452 \\
\hline dublīn_cp2 & .1604798 & .1420927 & 1.13 & 0.281 & -.1491136 & .4700732 \\
\hline dublin_ḡgdd2 & -.9177348 & .4367912 & $-2 \cdot 10$ & 0.057 & -1.869421 & .0339514 \\
\hline dublin_cpgdd & .6920435 & .4580961 & 1.51 & 0.157 & -.3060622 & 1.690149 \\
\hline dublin_ndv $\sim p$ & -51.01865 & 38.77182 & -1.32 & 0.213 & -135.4952 & 33.4579 \\
\hline dublin_ndv $\sim d$ & -761.5912 & 387.4229 & -1.97 & 0.073 & -1605.713 & 82.53072 \\
\hline _cons & -2378.104 & 1817.023 & -1.31 & 0.215 & -6337.058 & 1580.85 \\
\hline
\end{tabular}

Booneville, MS

Linear regression

$\begin{array}{lrr}\text { Number of obs } & & 22 \\ \text { F }(6, \quad 15) & 0.71 \\ \text { Prob }>\text { F } & =.6477 \\ \text { R-squared } & =0.1725 \\ \text { Root MSE } & =5.5506\end{array}$

\begin{tabular}{|c|c|c|c|c|c|c|}
\hline booneville s & Coef. & $\begin{array}{l}\text { Robust } \mathrm{HC} 3 \\
\text { Std. Err. }\end{array}$ & $t$ & $P>|t|$ & [95\% Conf. & Interval] \\
\hline booneville i & 266.7389 & 1459.081 & 0.18 & 0.857 & -2843.218 & 3376.696 \\
\hline $\begin{array}{l}\text { boonevil__cp } \\
\text { boonevi _gdd }\end{array}$ & $\begin{array}{l}-.1435841 \\
\text { (dropped) }\end{array}$ & 5.550685 & -0.03 & 0.980 & -11.97459 & 11.68742 \\
\hline boonevilla i2 & -164.7805 & 905.3378 & -0.18 & 0.858 & -2094.462 & 1764.901 \\
\hline $\begin{array}{l}\text { boonevill p } 2 \\
\text { boonevill d2 }\end{array}$ & $\begin{array}{l}-.0140568 \\
\text { (dropped) }\end{array}$ & .0394153 & -0.36 & 0.726 & -.0980685 & .069955 \\
\hline boonevi pgdd & -.0459089 & .0581607 & -0.79 & 0.442 & -.1698754 & .0780577 \\
\hline $\begin{array}{l}\text { boonevil icp } \\
\text { boonevi igdd }\end{array}$ & $\begin{array}{r}1.176442 \\
\text { (dropped) }\end{array}$ & 7.200105 & 0.16 & 0.872 & -14.17022 & 16.5231 \\
\hline cons & -93.68471 & 586.8226 & -0.16 & 0.875 & -1344.467 & 1157.098 \\
\hline
\end{tabular}


Water Valley, MS

Linear regression

$\begin{array}{llr}\text { Number of obs } & = & 21 \\ \mathrm{~F}(9, \quad 11) & 3.21 \\ \text { Prob }>\text { F } & = & 0.0361 \\ \text { R-squared } & = & 0.4813 \\ \text { Root MSE } & = & 5.169\end{array}$

\begin{tabular}{|c|c|c|c|c|c|c|}
\hline watervalle s & Coef. & $\begin{array}{c}\text { Robust } \mathrm{HC} 3 \\
\text { Std. Err. }\end{array}$ & $t$ & $P>|t|$ & [95\% Conf. & Interval] \\
\hline atervalle i & -269.3815 & 3079.95 & -0.09 & 0.932 & -7048.306 & 6509.543 \\
\hline aterval _cp & -5.802061 & 15.01932 & -0.39 & 0.707 & -38.85937 & 27.25524 \\
\hline aterva $\bar{g}$ dd & -14.12001 & 47.64336 & -0.30 & 0.772 & -118.9823 & 90.74232 \\
\hline waterval̄ $\sim$ i2 & -14.14096 & 1776.318 & -0.01 & 0.994 & -3923.792 & 3895.51 \\
\hline atervall p2 & -.0406434 & .0723927 & -0.56 & 0.586 & -.1999787 & .1186919 \\
\hline atervall d 2 & -.0608461 & .3821662 & -0.16 & 0.876 & -.9019882 & .780296 \\
\hline aterva pgdd & .0706527 & .3314083 & 0.21 & 0.835 & -.6587721 & .8000776 \\
\hline aterval icp & 9.098075 & 17.17305 & 0.53 & 0.607 & -28.69955 & 46.8957 \\
\hline aterva igdd & 19.31529 & 56.29443 & 0.34 & 0.738 & -104.5879 & 143.2185 \\
\hline cons & 219.3205 & 1352.257 & 0.16 & 0.874 & -2756.977 & 3195.618 \\
\hline
\end{tabular}

Batesville, MS

Linear regression

$\begin{array}{lrr}\text { Number of obs } & & 22 \\ \mathrm{~F}(5, \quad 16) & =0.75 \\ \text { Prob }>\text { F } & = & 0.5997 \\ \text { R-squared } & =0.2051 \\ \text { Root MSE } & =5.4163\end{array}$

\begin{tabular}{|c|c|c|c|c|c|c|}
\hline batesville s & Coef. & $\begin{array}{l}\text { Robust HC3 } \\
\text { Std. Err. }\end{array}$ & $t$ & $P>|t|$ & [95\% Conf. & Interval] \\
\hline batesville i & -1045.427 & 3853.214 & -0.27 & 0.790 & -9213.877 & 7123.022 \\
\hline batesvil _cp & -17.19772 & 24.11647 & -0.71 & 0.486 & -68.32236 & 33.92692 \\
\hline batesvi _ōgdd & (dropped) & & & & & \\
\hline batesvill i2 & 503.1346 & 2491.572 & 0.20 & 0.843 & -4778.762 & 5785.031 \\
\hline batesvill p2 & .0210067 & .112238 & 0.19 & 0.854 & -.2169272 & .2589405 \\
\hline batesvill d2 & (dropped) & & & & & \\
\hline batesvi pgdd & (dropped) & & & & & \\
\hline batesvil icp & 24.06394 & 31.24667 & 0.77 & 0.452 & -42.17604 & 90.30391 \\
\hline batesvi igdd & (dropped) & & & & & \\
\hline _cons & 508.6678 & 1502.459 & 0.34 & 0.739 & -2676.402 & 3693.738 \\
\hline
\end{tabular}

Yazoo City, MS

Linear regression

$\begin{array}{llr}\text { Number of obs } & & 22 \\ \text { F }(9, \quad 12) & 1.62 \\ \text { Prob }>\text { F } & =0.2148 \\ \text { R-squared } & =0.3863 \\ \text { Root MSE } & =6.6943\end{array}$

\begin{tabular}{|c|c|c|c|c|c|c|}
\hline \multirow[b]{2}{*}{ yazoocitym s } & \multicolumn{3}{|c|}{ Robust $\mathrm{HC} 3$} & \multirow[b]{2}{*}{$P>|t|$} & \multirow[b]{2}{*}{ [95\% Conf. } & \multirow[b]{2}{*}{ Interval ] } \\
\hline & Coef. & Std. Err. & $t$ & & & \\
\hline yazoocitym i & 2961.251 & 3100.353 & 0.96 & 0.358 & -3793.837 & 9716.34 \\
\hline yazoo_cp & 26.48203 & 30.31091 & 0.87 & 0.399 & -39.55978 & 92.52383 \\
\hline yazoo_ōgdd & .4270464 & 7.059756 & 0.06 & 0.953 & -14.95484 & 15.80893 \\
\hline yazoo_ndvi2 & -1596.547 & 1750.351 & -0.91 & 0.380 & -5410.235 & 2217.14 \\
\hline yazōo_cp2 & -.1736957 & .2061543 & -0.84 & 0.416 & -.6228672 & .2754759 \\
\hline yazoo_gdd2 & .0047357 & .0091654 & 0.52 & 0.615 & -.015234 & .0247054 \\
\hline yazoo_cpgdd & -.0454359 & .1386052 & -0.33 & 0.749 & -.3474307 & .2565589 \\
\hline yazoo_ndvicp & -26.57372 & 33.68391 & -0.79 & 0.445 & -99.96465 & 46.81721 \\
\hline yazoo_ndvi d & -.3119233 & 8.197144 & -0.04 & 0.970 & -18.17197 & 17.54812 \\
\hline _cons & -1352.838 & 1383.529 & -0.98 & 0.347 & -4367.289 & 1661.613 \\
\hline
\end{tabular}


Du Quoin, IL

Linear regression

$\begin{array}{lrr}\text { Number of obs } & & 22 \\ \text { F }(9,12) & 0.76 \\ \text { Prob }>\text { F } & = & 0.6505 \\ \text { R-squared } & =0.6216 \\ \text { Root MSE } & =4.2006\end{array}$

\begin{tabular}{|c|c|c|c|c|c|c|}
\hline duquoinil_ s & Coef. & $\begin{array}{c}\text { Robust } \mathrm{HC} 3 \\
\text { Std. Err. }\end{array}$ & t & $P>|t|$ & [ $95 \%$ Conf. & Int \\
\hline duquoinil_i & -713.8856 & 702.4978 & -1.02 & 0.330 & -2244.497 & 816.7255 \\
\hline duquoin_cp & -18.26719 & 11.44788 & -1.60 & 0.137 & -43.20998 & 6.675602 \\
\hline duquoin_ḡdd & -3.011217 & 4.246012 & -0.71 & 0.492 & -12.26248 & 6.240049 \\
\hline duquoin_nd 2 & 257.6696 & 369.3275 & 0.70 & 0.499 & -547.0259 & 1062.365 \\
\hline duquoiñ_cp2 & .0727957 & .0576295 & 1.26 & 0.231 & -.0527683 & .1983597 \\
\hline duquoin_ḡdd2 & -.0025192 & .0060473 & -0.42 & 0.684 & -.015695 & .0106566 \\
\hline duquoin_cp d & -.0005483 & .0289758 & -0.02 & 0.985 & -.0636811 & .0625845 \\
\hline duquoin_nd p & 22.70392 & 13.63845 & 1.66 & 0.122 & -7.011703 & 52.41954 \\
\hline duquoin_nd d & 4.333074 & 5.443689 & 0.80 & 0.442 & -7.527706 & 16.19385 \\
\hline _cons & 418.158 & 338.3439 & 1.24 & 0.240 & -319.0299 & 1155.346 \\
\hline
\end{tabular}

Minonk, IL

Linear regression

$\begin{array}{llr}\text { Number of obs } & & 22 \\ \text { F }(9, \quad 12) & 0.88 \\ \text { Prob }>\text { F } & =0.5649 \\ \text { R-squared } & =0.7343 \\ \text { Root MSE } & =4.4289\end{array}$

\begin{tabular}{|c|c|c|c|c|c|c|}
\hline minonkil_y s & Coef. & $\begin{array}{c}\text { Robust HC3 } \\
\text { Std. Err. }\end{array}$ & $t$ & $P>|t|$ & [ $95 \%$ Conf. & Interval] \\
\hline minonkil_m i & 670.8499 & 2298.043 & 0.29 & 0.775 & -4336.157 & 5677.856 \\
\hline minon $\bar{k} \_c p$ & 8.917755 & 25.18459 & 0.35 & 0.729 & -45.95475 & 63.79026 \\
\hline minonk_ogdd & -203.8351 & 113101.6 & -0.00 & 0.999 & -246631 & 246223.3 \\
\hline minonk_ndvi2 & -396.3504 & 1535.588 & -0.26 & 0.801 & -3742.11 & 2949.409 \\
\hline minonk_cp2 & -.1730022 & .1221612 & -1.42 & 0.182 & -.4391687 & .0931642 \\
\hline minonk_ḡdd2 & 4.135079 & 2336.167 & 0.00 & 0.999 & -5085.935 & 5094.205 \\
\hline minonk_cpgdd & -3.840381 & 1070.732 & -0.00 & 0.997 & -2336.765 & 2329.085 \\
\hline minonk_ndv $\sim$ p & -4.053321 & 28.95428 & -0.14 & 0.891 & -67.13929 & 59.03264 \\
\hline minonk_ndv $\sim \mathrm{d}$ & 278.4009 & 136992.8 & 0.00 & 0.998 & -298203.2 & 298760 \\
\hline cons & -276.0849 & 836.5011 & -0.33 & 0.747 & -2098.664 & 1546.494 \\
\hline
\end{tabular}

Windsor, IL

Linear regression

$\begin{array}{llr}\text { Number of obs } & & 22 \\ \text { F }(9,12) & 4.84 \\ \text { Prob }>\text { F } & = & 0.0067 \\ \text { R-squared } & = & 0.6746 \\ \text { Root MSE } & = & 4.3463\end{array}$

\begin{tabular}{|c|c|c|c|c|c|c|}
\hline windsoril_ s & Coef. & $\begin{array}{l}\text { Robust } \mathrm{HC} 3 \\
\text { Std. Err. }\end{array}$ & $t$ & $P>|t|$ & [95\% Conf. & Interval] \\
\hline windsoril_i & 515.2734 & 724.6016 & 0.71 & 0.491 & -1063.498 & 2094.045 \\
\hline windsor_cp & 4.313011 & 15.71782 & 0.27 & 0.788 & -29.93318 & 38.5592 \\
\hline windsor_gdd & -.0790699 & .3274609 & -0.24 & 0.813 & -.7925459 & .6344062 \\
\hline windsor_nd 2 & -244.5751 & 557.5881 & -0.44 & 0.669 & -1459.455 & 970.305 \\
\hline windsor_cp2 & .0507554 & .2178401 & 0.23 & 0.820 & -.4238775 & .5253882 \\
\hline windsor_gadd2 & .0000588 & .0002837 & 0.21 & 0.839 & -.0005595 & .000677 \\
\hline windsor_cp d & -.0021712 & .012079 & -0.18 & 0.860 & -.0284891 & .0241468 \\
\hline windsor_nd p & -6.399578 & 18.31468 & -0.35 & 0.733 & -46.30383 & 33.50468 \\
\hline windsor_nd d & .0670142 & .3631861 & 0.18 & 0.857 & -.7243004 & .8583289 \\
\hline cons & -207.2024 & 245.2546 & -0.84 & 0.415 & -741.5662 & 327.1614 \\
\hline
\end{tabular}


White Hall, IL

Linear regression

$\begin{array}{llr}\text { Number of obs } & & 22 \\ \text { F }(7, \quad 13) & = \\ \text { Prob }>\text { F } & = & \dot{ } \\ \text { R-squared } & = & 0.6650 \\ \text { Root MSE } & = & 4.4317\end{array}$

\begin{tabular}{|c|c|c|c|c|c|c|}
\hline whitehalli s & Coef. & $\begin{array}{l}\text { Robust } \mathrm{HC} 3 \\
\text { Std. Err. }\end{array}$ & $t$ & $P>|t|$ & [ $95 \%$ Conf. & Interval] \\
\hline whitehalli i & 878.775 & 1412.078 & 0.62 & 0.544 & -2171.835 & 3929.385 \\
\hline whitehall_cp & -4.779675 & 9.90794 & -0.48 & 0.638 & -26.18448 & 16.62513 \\
\hline whiteha _ōgdd & -20.30925 & 21.71641 & -0.94 & 0.367 & -67.22471 & 26.6062 \\
\hline whitehal $\bar{l} \sim i 2$ & -634.1398 & 963.7031 & -0.66 & 0.522 & -2716.094 & 1447.814 \\
\hline whitehall p2 & -.0540039 & .1078244 & -0.50 & 0.625 & -.2869444 & .1789366 \\
\hline whitehall d2 & .0135137 & .0144931 & 0.93 & 0.368 & -.0177968 & .0448241 \\
\hline whiteha pgdd & 1.182473 & 1.264952 & 0.93 & 0.367 & -1.55029 & 3.915235 \\
\hline whitehal icp & 8.22195 & 14.08246 & 0.58 & 0.569 & -22.20135 & 38.64525 \\
\hline $\begin{array}{r}\text { whiteha igdd } \\
\text { cons }\end{array}$ & $\begin{array}{l}\text { (dropped) } \\
-267.4864\end{array}$ & 520.5278 & -0.51 & 0.616 & -1392.018 & 857.0455 \\
\hline
\end{tabular}

\section{Beaverdam, KY}

Linear regression

$\begin{array}{llr}\text { Number of obs } & = & 22 \\ \text { F }(9, \quad 12) & =0.86 \\ \text { Prob }>\text { F } & =0.5801 \\ \text { R-squared } & =0.3695 \\ \text { Root MSE } & =5.7045\end{array}$

\begin{tabular}{|c|c|c|c|c|c|c|}
\hline & & Robust $\mathrm{HC} 3$ & & & & \\
\hline beaverdamk s & Coef. & Std. Err. & $t$ & $P>|t|$ & [ $95 \%$ Conf. & Inte \\
\hline beaverdamk i & 348.2916 & 6138.431 & 0.06 & 0.956 & -13026.2 & 13722.78 \\
\hline beaverdam_cp & 9.022764 & 51.30345 & 0.18 & 0.863 & -102.7578 & 120.8034 \\
\hline beaverd _ $\bar{g}$ dd & -.1677813 & 2.188623 & -0.08 & 0.940 & -4.936381 & 4.600819 \\
\hline beaverdam i2 & -137.9155 & 4597.251 & -0.03 & 0.977 & -10154.47 & 9878.635 \\
\hline beaverdam p2 & -.0314492 & .2644391 & -0.12 & 0.907 & -.6076124 & .5447141 \\
\hline beaverdam d2 & -.0000743 & .0006103 & -0.12 & 0.905 & -.001404 & .0012554 \\
\hline beaverd pgdd & .0050291 & .0341503 & 0.15 & 0.885 & -.0693781 & .0794364 \\
\hline beaverda icp & -12.81797 & 50.81014 & -0.25 & 0.805 & -123.5237 & 97.88782 \\
\hline beaverd igdd & .1739356 & 2.223353 & 0.08 & 0.939 & -4.670334 & 5.018206 \\
\hline _cons & -136.1653 & 1945.236 & -0.07 & 0.945 & -4374.47 & 4102.139 \\
\hline
\end{tabular}

\section{Providence, KY}

Linear regression

$\begin{array}{llr}\text { Number of obs } & & 22 \\ \text { F }(9, \quad 12) & 1.55 \\ \text { Prob }>\text { F } & = & 0.2356 \\ \text { R-squared } & = & 0.5378 \\ \text { Root MSE } & = & 5.6177\end{array}$

\begin{tabular}{|c|c|c|c|c|c|c|}
\hline providence s & Coef. & $\begin{array}{l}\text { Robust HC3 } \\
\text { Std. Err. }\end{array}$ & t & $P>|t|$ & [95\% Conf. & Interval ] \\
\hline providence i & 990.681 & 6629.011 & 0.15 & 0.884 & -13452.69 & 15434.05 \\
\hline providence p & 32.27764 & 60.82382 & 0.53 & 0.605 & -100.2461 & 164.8014 \\
\hline providn _ gdd & -.1365521 & .8512542 & -0.16 & 0.875 & -1.991276 & 1.718172 \\
\hline providnec $\sim$ i2 & -302.3633 & 4577.627 & -0.07 & 0.948 & -10276.16 & 9671.43 \\
\hline providnec $\sim$ p2 & .0466135 & .1637737 & 0.28 & 0.781 & -.3102188 & .4034457 \\
\hline providnec $\sim \mathrm{d} 2$ & .0000157 & .000043 & 0.37 & 0.721 & -.000078 & .0001095 \\
\hline providn pgdd & .0016524 & .0033279 & 0.50 & 0.628 & -.0055985 & .0089033 \\
\hline providnece $\sim$ p & -45.22737 & 85.21806 & -0.53 & 0.605 & -230.9016 & 140.4468 \\
\hline providn igdd & .1297933 & 1.126343 & 0.12 & 0.910 & -2.324297 & 2.583884 \\
\hline -cons & -520.7356 & 517.739 & -0.21 & 0.840 & -6006.418 & 4964.947 \\
\hline
\end{tabular}


Farmville, VA

Linear regression

$\begin{array}{llr}\text { Number of obs } & & 9 \\ \text { F }(5, & 0) & = \\ \text { Prob }>\text { F } & = & \text {. } \\ \text { R-squared } & = & 1.0000 \\ \text { Root MSE } & = & 0\end{array}$

\begin{tabular}{|c|c|c|c|c|c|c|c|}
\hline farmvillev s & Coef. & $\begin{array}{c}\text { Robust } \mathrm{HC} 3 \\
\text { Std. Err. }\end{array}$ & $t$ & $P>|t|$ & {$[95 \%$} & Conf. & Interval] \\
\hline farmvillev i & -6873.861 & 3006.939 & -2.29 & . & & • & . \\
\hline farmville_cp & -194.4727 & 91.41609 & -2.13 & . & & . & . \\
\hline farmvil $\bar{g}$ dd & -9.836454 & 4.146786 & -2.37 & • & & . & . \\
\hline farmville $\sim i 2$ & (dropped) & & & & & & \\
\hline farmville p2 & 1.416668 & .5353095 & 2.65 & - & & $\cdot$ & . \\
\hline farmville d2 & -.0001984 & .0001258 & -1.58 & $\cdot$ & & . & . \\
\hline farmvil pgdd & .0587549 & .0187169 & 3.14 & $\cdot$ & & . & . \\
\hline farmvill icp & 176.0518 & 88.95232 & 1.98 & $\bullet$ & & . & . \\
\hline farmvil igdd & 11.54697 & 4.973281 & 2.32 & . & & . & . \\
\hline cons & 5964.722 & 2572.438 & 2.32 & . & & . & . \\
\hline
\end{tabular}

\section{Menomonie, WI}

Linear regression

$\begin{array}{llr}\text { Number of obs } & = & 22 \\ \mathrm{~F}(9, \quad 12) & = & 1.99 \\ \text { Prob }>\text { F } & = & 0.1328 \\ \text { R-squared } & =0.4080 \\ \text { Root MSE } & =7.0307\end{array}$

\begin{tabular}{|c|c|c|c|c|c|c|}
\hline menomoniew s & Coef. & $\begin{array}{c}\text { Robust HC } 3 \\
\text { Std. Err. }\end{array}$ & $t$ & $P>|t|$ & [95\% Conf. & val ] \\
\hline enomoniew i & -5545.201 & 5578.198 & -0.99 & 0.340 & -17699.05 & 6608.649 \\
\hline enomonie_cp & 1.3357 & 50.21382 & 0.03 & 0.979 & -108.0708 & 110.7422 \\
\hline enomon $\sim \bar{g} \mathrm{dd}$ & .4125578 & 1.451487 & 0.28 & 0.781 & -2.74996 & 3.575076 \\
\hline enomonie $i 2$ & 3518.649 & 3664.462 & 0.96 & 0.356 & -4465.528 & 11502.83 \\
\hline nonie p2 & -.2518738 & 4623 & -0.68 & 0.508 & -1.056863 & .5531154 \\
\hline nomonie d2 & -.000407 & .0004183 & -0.97 & 0.350 & -.0013183 & .0005044 \\
\hline enomon $\sim$ pgdd & -.0158651 & .0217627 & -0.73 & 0.480 & -.0632819 & .0315517 \\
\hline enomoni icp & 11.22641 & 51.77995 & 0.22 & 0.832 & -101.5924 & 124.0452 \\
\hline nomon igdd & -.0684475 & 1.709388 & -0.04 & 0.969 & -3.792885 & 3.65599 \\
\hline _cons & 2115.806 & 2135.027 & 0.99 & 0.341 & -2536.018 & 6767.629 \\
\hline
\end{tabular}

\section{Arlington, WI}

Linear regression

$\begin{array}{llr}\text { Number of obs } & & 22 \\ \text { F }(9, \quad 12) & 0.50 \\ \text { Prob }>\text { F } & =0.8463 \\ \text { R-squared } & =0.3652 \\ \text { Root MSE } & =6.5359\end{array}$

\begin{tabular}{|c|c|c|c|c|c|c|}
\hline arlingtonw s & Coef. & $\begin{array}{c}\text { Robust } \mathrm{HC} 3 \\
\text { Std. Err. }\end{array}$ & $t$ & $P>|t|$ & [95\% Conf. & Interval] \\
\hline arlingtonw i & -1271.271 & 3540.671 & -0.36 & 0.726 & -8985.731 & 6443.189 \\
\hline rlington $\mathrm{cp}$ & -8.880773 & 42.10382 & -0.21 & 0.836 & -100.6171 & 82.85557 \\
\hline rlingt _ $\bar{g} d \bar{d}$ & -.484403 & 1.945659 & -0.25 & 0.808 & -4.723629 & 3.754823 \\
\hline rlington $\sim$ i2 & 654.0308 & 2208.43 & 0.30 & 0.772 & -4157.725 & 5465.787 \\
\hline arlington $\sim \mathrm{p} 2$ & -.081256 & .2799215 & -0.29 & 0.777 & -.6911525 & .5286405 \\
\hline arlington d2 & .0001127 & .0009327 & 0.12 & 0.906 & -.0019195 & .0021449 \\
\hline rlingt pgdd & .0021839 & .0426298 & 0.05 & 0.960 & -.0906984 & .0950662 \\
\hline arlingto icp & 14.4348 & 41.9 & 0.34 & 0.737 & -77.04022 & 9098 \\
\hline arlingt igdd & .5167096 & 2.033633 & 0.25 & 0.804 & -3.914197 & 4.947616 \\
\hline cons & 619.9949 & 1490.736 & 0.42 & 0.685 & -2628.039 & 3868.029 \\
\hline
\end{tabular}


Sellinggrove, PA

Linear regression

$\begin{array}{lrr}\text { Number of obs } & = & 21 \\ \mathrm{~F}(5, \quad 15) & =0.93 \\ \text { Prob }>\mathrm{F} & = & 0.4916 \\ \text { R-squared } & = & 0.2336 \\ \text { Root MSE } & = & 6.0537\end{array}$

\begin{tabular}{|c|c|c|c|c|c|c|}
\hline sellingsgr s & Coef. & $\begin{array}{l}\text { Robust } \mathrm{HC} 3 \\
\text { Std. Err. }\end{array}$ & $t$ & $P>|t|$ & [95\% Conf. & Interval] \\
\hline elingsgro i & 1404.441 & 3819.156 & 0.37 & 0.718 & -6735.898 & 9544.779 \\
\hline $\begin{array}{l}\text { selingsgro p } \\
\text { selling _ gdd }\end{array}$ & $\begin{array}{r}9.932671 \\
\text { (dropped) }\end{array}$ & 19.36688 & 0.51 & 0.616 & -31.34686 & 51.2122 \\
\hline ellingsg i2 & -824.2485 & 2495.248 & -0.33 & 0.746 & -6142.744 & 4494.247 \\
\hline ellingsg p2 & -.0234761 & .1513875 & -0.16 & 0.879 & -.3461508 & .2991987 \\
\hline ellingsg d 2 & (dropped) & & & & & \\
\hline $\begin{array}{l}\text { elling pgdd } \\
\text { ellingsgr } ~\end{array}$ & $\begin{array}{l}\text { (dropped) } \\
-10.97639\end{array}$ & 24.10172 & -0.46 & 0.655 & -62.34799 & 40.39522 \\
\hline lling igdd & (dropped) & & & & & \\
\hline _cons & -574.0845 & 1481.091 & -0.39 & 0.704 & -3730.956 & 2582.787 \\
\hline
\end{tabular}

Regression results for Wheat Yields

Saluda, SC

Linear regression

$\begin{array}{llr}\text { Number of obs } & & 22 \\ \text { F } 7, \quad 13) & . \\ \text { Prob }>\text { F } & = & . \\ \text { R-squared } & = & 0.5391 \\ \text { Root MSE } & = & 4.9686\end{array}$

\begin{tabular}{|c|c|c|c|c|c|c|}
\hline saluda_yie s & Coef. & $\begin{array}{c}\text { Robust } \mathrm{HC} 3 \\
\text { Std. Err. }\end{array}$ & t & $P>|t|$ & $\mathrm{hf}$. & Int \\
\hline saludasc_m i & 301.3616 & 6845.909 & 0.04 & 0.966 & -14488.33 & 15091.05 \\
\hline saludā_cp & 13.54305 & 15.96442 & 0.85 & 0.412 & -20.94599 & 48.03209 \\
\hline saluda_gdd & 2.489201 & 3.610653 & 0.69 & 0.503 & -5.311139 & 10.28954 \\
\hline saluda_nd̄vi2 & -53.42181 & 4650.756 & -0.01 & 0.991 & -10100.77 & 9993.925 \\
\hline salud̄a_cp2 & -.0464149 & .085229 & -0.54 & 0.595 & -.2305409 & .1377112 \\
\hline saluda_gdd2 & -.0226086 & .1375451 & -0.16 & 0.872 & -.3197568 & .2745395 \\
\hline saluda_cpgdd & -.1606883 & .3117562 & -0.52 & 0.615 & -.8341968 & .5128201 \\
\hline saluda_ndv $\sim p$ & -15.24993 & 21.36448 & -0.71 & 0.488 & -61.40508 & 30.90522 \\
\hline cons & -180.4933 & 2513.745 & -0.07 & 0.944 & -5611.108 & 5250.122 \\
\hline
\end{tabular}


Dublin, GA

Linear regression

$\begin{array}{llr}\text { Number of obs } & & 22 \\ \mathrm{~F}(9, \quad 12) & 99.17 \\ \text { Prob }>\text { F } & =0.0000 \\ \text { R-squared } & =0.4726 \\ \text { Root MSE } & =8.7332\end{array}$

\begin{tabular}{|c|c|c|c|c|c|c|}
\hline dublinga_y s & Coef. & $\begin{array}{c}\text { Robust } \mathrm{HC} 3 \\
\text { Std. Err. }\end{array}$ & $t$ & $P>|t|$ & [95\% Conf. & Interval] \\
\hline dublinga_m i & 2691.782 & 10503.13 & 0.26 & 0.802 & -20192.57 & 25576.14 \\
\hline dublin_ $\mathrm{cp}$ & 19.03704 & 42.81539 & 0.44 & 0.664 & -74.24968 & 112.3238 \\
\hline dublin_ōgdd & -332.8699 & 406.2263 & -0.82 & 0.429 & -1217.961 & 552.2212 \\
\hline dublin_ndvi2 & -1930.572 & 8175.062 & -0.24 & 0.817 & -19742.5 & 15881.36 \\
\hline dublīn_cp2 & .0391457 & .5207775 & 0.08 & 0.941 & -1.095531 & 1.173822 \\
\hline dublin_gdd2 & 1.277257 & .7956326 & 1.61 & 0.134 & -.4562773 & 3.010792 \\
\hline dublin_cpgdd & -.9521408 & .9779746 & -0.97 & 0.349 & -3.082964 & 1.178683 \\
\hline dublin ndv $\sim p$ & -30.00433 & 74.32729 & -0.40 & 0.694 & -191.9496 & 131.9409 \\
\hline ublin_ndv $\sim d$ & 510.6074 & 635.1212 & 0.80 & 0.437 & -873.2028 & 1894.418 \\
\hline -_cons & -888.076 & 3399.29 & -0.26 & 0.798 & -8294.493 & 6518.341 \\
\hline
\end{tabular}

Booneville, MS

Linear regression

$\begin{array}{lrr}\text { Number of obs } & = & 12 \\ \mathrm{~F}(5, & 6) & 0.49 \\ \text { Prob }>\text { F } & = & 0.7761 \\ \text { R-squared } & = & 0.8222 \\ \text { Root MSE } & = & 4.0751\end{array}$

\begin{tabular}{|c|c|c|c|c|c|c|}
\hline booneville s & Coef. & $\begin{array}{l}\text { Robust HC3 } \\
\text { Std. Err. }\end{array}$ & $t$ & $P>|t|$ & [95\% Conf. & Interval ] \\
\hline ooneville i & -2094.375 & 5193.735 & -0.40 & 0.701 & -14802.99 & 10614.24 \\
\hline $\begin{array}{l}\text { boonevil _cp } \\
\text { boonevi - gdd }\end{array}$ & $\begin{array}{l}-9.280433 \\
\text { (dropped) }\end{array}$ & 29.77957 & -0.31 & 0.766 & -82.14842 & 63.58756 \\
\hline boonevil̄ i2 & 1287.119 & 3307.583 & 0.39 & 0.711 & -6806.246 & 9380.483 \\
\hline $\begin{array}{l}\text { boonevill p2 } \\
\text { boonevill d2 } \\
\text { boonevi pgdd }\end{array}$ & $\begin{array}{l}-.0261649 \\
\text { (dropped) } \\
\text { (dropped) }\end{array}$ & .2096229 & -0.12 & 0.905 & -.5390937 & .4867639 \\
\hline 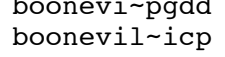 & $\begin{array}{r}\text { (aropped) } \\
13.57053\end{array}$ & 34.14473 & 0.40 & 0.705 & -69.97861 & 97.11967 \\
\hline Donevi igdd & (dropped) & & & & & \\
\hline _cons & 870.1062 & 2038.994 & 0.43 & 0.684 & -4119.133 & 5859.345 \\
\hline
\end{tabular}

Batesville, MS

Linear regression

$\begin{array}{llr}\text { Number of obs } & & 22 \\ \text { F }(5, \quad 16) & =0.92 \\ \text { Prob }>\text { F } & =0.4910 \\ \text { R-squared } & =0.3107 \\ \text { Root MSE } & =8.0366\end{array}$

\begin{tabular}{|c|c|c|c|c|c|c|}
\hline batesville s & Coef. & $\begin{array}{l}\text { Robust HC3 } \\
\text { Std. Err. }\end{array}$ & $t$ & $P>|t|$ & [95\% Conf. & Interval] \\
\hline batesville i & -3340.344 & 5195.109 & -0.64 & 0.529 & -14353.48 & 7672.794 \\
\hline $\begin{array}{l}\text { batesvil_ cp } \\
\text { batesvi _gdd }\end{array}$ & $\begin{array}{l}-24.41902 \\
\text { (dropped) }\end{array}$ & 32.51311 & -0.75 & 0.464 & -93.34374 & 44.5057 \\
\hline atesvill $\sim$ i2 & 2174.003 & 3390.088 & 0.64 & 0.530 & -5012.661 & 9360.668 \\
\hline $\begin{array}{l}\text { batesvill p } 2 \\
\text { batesvill d2 }\end{array}$ & $\begin{array}{r}.1654422 \\
\text { (dropped) }\end{array}$ & .1431172 & 1.16 & 0.265 & -.1379528 & .4688372 \\
\hline atesvi pgdd & (dropped) & & & & & \\
\hline $\begin{array}{l}\text { patesvil icp } \\
\text { patesvi igdd }\end{array}$ & $\begin{array}{r}26.98684 \\
\text { (dropped) }\end{array}$ & 41.96313 & 0.64 & 0.529 & -61.97102 & 115.9447 \\
\hline _cons & 1355.375 & 2012.384 & 0.67 & 0.510 & -2910.688 & 5621.439 \\
\hline
\end{tabular}


Yazoo City, MS

Linear regression

$\begin{array}{llr}\text { Number of obs } & & 22 \\ \text { F }(9,12) & 1.02 \\ \text { Prob }>\text { F } & = & 0.4771 \\ \text { R-squared } & =0.5186 \\ \text { Root MSE } & =9.1498\end{array}$

\begin{tabular}{|c|c|c|c|c|c|c|}
\hline yazoocitym s & Coef. & $\begin{array}{l}\text { Robust } \mathrm{HC} 3 \\
\text { Std. Err. }\end{array}$ & t & $P>|t|$ & [95\% Conf. & Interval] \\
\hline azoocitym i & -3813.209 & 6057.574 & -0.63 & 0.541 & -17011.53 & 9385.112 \\
\hline yazoo_cp & -35.09098 & 62.44078 & -0.56 & 0.584 & -171.1378 & 100.9558 \\
\hline yazoo_gdd & -8.446845 & 16.34374 & -0.52 & 0.615 & -44.05679 & 27.1631 \\
\hline yazoo_ndvi2 & 1972.449 & 3666.062 & 0.54 & 0.600 & -6015.214 & 9960.112 \\
\hline yazoo_cp2 & .0807863 & .3074945 & 0.26 & 0.797 & -.5891866 & .7507593 \\
\hline yazoo_ḡdd2 & .0037624 & .0139535 & 0.27 & 0.792 & -.0266397 & .0341644 \\
\hline yazoo_cpgdd & -.0336206 & .2623916 & -0.13 & 0.900 & -.6053228 & .5380817 \\
\hline yazoo_ndvicp & 39.8859 & 75.8576 & 0.53 & 0.609 & -125.3936 & 205.1654 \\
\hline yazoo_ndvi d & 11.13524 & 20.45858 & 0.54 & 0.596 & -33.44018 & 55.71066 \\
\hline _cons & 1854.543 & 2571.649 & 0.72 & 0.485 & -3748.6 & 7457.686 \\
\hline
\end{tabular}

Angelica, NY

Linear regression

$\begin{array}{llr}\text { Number of obs } & & 12 \\ \mathrm{~F}(0, & 2) & \\ \text { Prob }>\text { F } & = & . \\ \text { R-squared } & = & 0.9510 \\ \text { Root MSE } & = & 4.9279\end{array}$

\begin{tabular}{|c|c|c|c|c|c|c|}
\hline angelicany s & Coef. & $\begin{array}{c}\text { Robust } \mathrm{HC} 3 \\
\text { Std. Err. }\end{array}$ & $t$ & $P>|t|$ & [95\% Conf. & Interval] \\
\hline angelicany i & 32716.55 & $\cdot$ & • & • & • & - \\
\hline angelica_cp & -138.0211 & . & . & . & • & . \\
\hline angelica_ḡd & -203.2377 & . & . & . & . & • \\
\hline angelica_n 2 & -20911.43 & . & . & . & . & • \\
\hline angelica_cp2 & -.5797227 & . & . & . & . & . \\
\hline angelica_g 2 & -.0667262 & . & • & - & - & - \\
\hline angelica_c d & .1524282 & • & . & . & . & 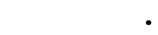 \\
\hline angelica_n p & 178.4685 & . & . & . & . & - \\
\hline angelica_n d & 239.6487 & . & - & - & - & - \\
\hline cons & -12713.71 & • & . & . & . & \\
\hline
\end{tabular}

\section{Riverhead, NY}

Linear regression

$\begin{array}{llr}\text { Number of obs } & & 20 \\ \text { F }(9, \quad 10) & 1.50 \\ \text { Prob }>\text { F } & =0.2685 \\ \text { R-squared } & =0.4483 \\ \text { Root MSE } & =6.6475\end{array}$

\begin{tabular}{|c|c|c|c|c|c|c|}
\hline riverheadn s & Coef. & $\begin{array}{l}\text { Robust HC3 } \\
\text { Std. Err. }\end{array}$ & t & $P>|t|$ & {$[95 \%$} & Interval] \\
\hline riverheadn i & -99.46671 & 1146.257 & -0.09 & 0.933 & -2653.486 & 2454.552 \\
\hline riverhead_cp & 1.939268 & 15.64271 & 0.12 & 0.904 & -32.91487 & 36.79341 \\
\hline riverhe gdd & -.117822 & .6746737 & -0.17 & 0.865 & -1.621089 & 1.385445 \\
\hline riverhead i2 & 317.1097 & 1013.354 & 0.31 & 0.761 & -1940.785 & 2575.004 \\
\hline riverhead p2 & -.0191752 & .1313136 & -0.15 & 0.887 & -.3117601 & .2734098 \\
\hline riverhead d2 & .0006334 & .0010753 & 0.59 & 0.569 & -.0017626 & .0030293 \\
\hline riverhe pgdd & .0039961 & .046261 & 0.09 & 0.933 & -.0990798 & .1070721 \\
\hline riverhea icp & -7.444091 & 38.43954 & -0.19 & 0.850 & -93.09273 & 78.20455 \\
\hline riverhe igdd & -.3657982 & 1.722204 & -0.21 & 0.836 & -4.203109 & 3.471512 \\
\hline _cons & 65.60401 & 262.8119 & 0.25 & 0.808 & -519.9775 & 651.1855 \\
\hline
\end{tabular}


Fredonia, NY

Linear regression

$\begin{array}{llr}\text { Number of obs } & & 9 \\ \text { F }(8, & 0) & \\ \text { Prob }>\text { F } & = & . \\ \text { R-squared } & = & 1.0000 \\ \text { Root MSE } & = & 0\end{array}$

\begin{tabular}{|c|c|c|c|c|c|c|c|}
\hline fredoniany $\sim$ s & Coef. & $\begin{array}{c}\text { Robust } \mathrm{HC} 3 \\
\text { Std. Err. }\end{array}$ & t & $P>|t|$ & {$[95 \%$} & Conf. & Interval] \\
\hline fredoniany i & (dropped) & & & & & & \\
\hline fredonia_cp & 86.47184 & 7.59977 & 11.38 & . & & . & . \\
\hline fredonia $\bar{g}$ dd & 10.39545 & .9644384 & 10.78 & • & & . & . \\
\hline fredonia_n 2 & 570.9995 & 64.90501 & 8.80 & . & & . & . \\
\hline fredonia_cp2 & -1.841552 & .1497037 & $-12 \cdot 30$ & . & & . & . \\
\hline fredonia-g 2 & -.0192242 & .0017692 & -10.87 & • & & . & . \\
\hline fredonia_c $\sim$ d & -.3950932 & .035417 & -11.16 & . & & . & . \\
\hline fredonia-n $n$ & -26.38387 & 3.148439 & -8.38 & • & & . & . \\
\hline fredonia_n d & -4.521798 & .4983747 & -9.07 & . & & . & . \\
\hline cons & -850.2493 & 78.63009 & -10.81 & . & & . & . \\
\hline
\end{tabular}

Du Quoin, IL

Linear regression

$\begin{array}{llr}\text { Number of obs } & & 22 \\ \text { F }(9, \quad 12) & =0.16 \\ \text { Prob }>\text { F } & =.9954 \\ \text { R-squared } & =0.3022 \\ \text { Root MSE } & =8.986\end{array}$

\begin{tabular}{|c|c|c|c|c|c|c|}
\hline duquoinil_ s & Coef. & $\begin{array}{l}\text { Robust } \mathrm{HC} 3 \\
\text { Std. Err. }\end{array}$ & $t$ & $P>|t|$ & [ $95 \%$ Conf. & Interval] \\
\hline duquoinil_ i & -522.0029 & 1479.224 & -0.35 & 0.730 & -3744.956 & 2700.9 \\
\hline duquoin_cp & 4.536545 & 23.73432 & 0.19 & 0.852 & -47.17609 & 56.24918 \\
\hline duquoin_ḡdd & .4496125 & 6.431619 & 0.07 & 0.945 & -13.56368 & 14.46291 \\
\hline duquoin_nd 2 & 409.5261 & 802.9698 & 0.51 & 0.619 & -1339.995 & 2159.047 \\
\hline duquoiñ_cp2 & -.0224119 & .1849499 & -0.12 & 0.906 & -.4253831 & .3805593 \\
\hline duquoin_ḡdd2 & -.0053295 & .0166891 & -0.32 & 0.755 & -.0416919 & .0310328 \\
\hline duquoin_cp d & -.0099594 & .0564957 & -0.18 & 0.863 & -.1330529 & .1131341 \\
\hline duquoin_nd $\sim p$ & -4.919432 & 25.18025 & -0.20 & 0.848 & -59.78249 & 49.94363 \\
\hline duquoin_nd d & -.0703154 & 7.511318 & -0.01 & 0.993 & -16.43607 & 16.29544 \\
\hline cons & 193.6238 & 699.4225 & 0.28 & 0.787 & -1330.287 & 1717.535 \\
\hline
\end{tabular}

\section{Minonk, IL}

Linear regression

$\begin{array}{llr}\text { Number of obs } & & 22 \\ \text { F }(9, \quad 12) & =0.30 \\ \text { Prob }>\text { F } & =0.9606 \\ \text { R-squared } & =0.3338 \\ \text { Root MSE } & =13.369\end{array}$

\begin{tabular}{|c|c|c|c|c|c|c|}
\hline minonkil_y s & Coef. & $\begin{array}{c}\text { Robust } \mathrm{HC} 3 \\
\text { Std. Err. }\end{array}$ & $t$ & $P>|t|$ & [95\% Conf. & Interval ] \\
\hline minonkil_m i & 974.0359 & 4064.652 & 0.24 & 0.815 & -7882.079 & 9830.151 \\
\hline minon $\bar{k} \_c p$ & 15.34935 & 49.56083 & 0.31 & 0.762 & -92.63442 & 123.3331 \\
\hline minonk_ōgdd & 47.55891 & 376364 & 0.00 & 1.000 & -819979.2 & 820074.3 \\
\hline minonk_ndvi2 & -525.0528 & 2767.307 & -0.19 & 0.853 & -6554.498 & 5504.392 \\
\hline minonk_cp2 & -.0451123 & .2102437 & -0.21 & 0.834 & -.5031938 & .4129693 \\
\hline minonk_gdd2 & -1.72273 & 7773.977 & -0.00 & 1.000 & -16939.76 & 16936.32 \\
\hline minonk_cpgdd & 1.713455 & 3563.036 & 0.00 & 1.000 & -7761.475 & 7764.902 \\
\hline minonk_ndv $\sim$ p & -17.00657 & 58.07126 & -0.29 & 0.775 & -143.533 & 109.5198 \\
\hline $\begin{array}{r}\text { minonk_ndv } \sim \mathrm{d} \\
\text { cons }\end{array}$ & $\begin{array}{l}-71.97292 \\
-392.7288\end{array}$ & $\begin{array}{l}455865.8 \\
1459.766\end{array}$ & $\begin{array}{l}-0.00 \\
-0.27\end{array}$ & $\begin{array}{l}1.000 \\
0.792\end{array}$ & $\begin{array}{l}-993318.3 \\
-3573.285\end{array}$ & $\begin{array}{l}993174.3 \\
2787.828\end{array}$ \\
\hline & & & & & & \\
\hline
\end{tabular}


Ardmore, SD

Linear regression

$\begin{array}{llr}\text { Number of obs } & & 22 \\ \text { F }(9, \quad 12) & =1.36 \\ \text { Prob }>\text { F } & = & 0.3036 \\ \text { R-squared } & =0.4486 \\ \text { Root MSE } & =5.8739\end{array}$

\begin{tabular}{|c|c|c|c|c|c|c|}
\hline ardmoresd_ s & Coef. & $\begin{array}{c}\text { Robust HC3 } \\
\text { Std. Err. }\end{array}$ & $t$ & $P>|t|$ & [95\% Conf. & Interval] \\
\hline ardmoresd_ i & 219.2954 & 587.5873 & 0.37 & 0.715 & -1060.947 & 1499.538 \\
\hline ardmore ${ }^{-} \mathrm{cp}$ & 3.163519 & 15.55935 & 0.20 & 0.842 & -30.7374 & 37.06444 \\
\hline ardmore_ḡdd & -.1853926 & 1.525915 & -0.12 & 0.905 & -3.510075 & 3.13929 \\
\hline ardmore $\overline{n d} \sim 2$ & -105.6863 & 362.2723 & -0.29 & 0.775 & -895.0099 & 683.6372 \\
\hline ardmore_e_cp2 & -.0467921 & .2367557 & -0.20 & 0.847 & -.5626385 & .4690542 \\
\hline ardmore $\overline{\text { gdd } 2}$ & .0008695 & .0032745 & 0.27 & 0.795 & -.006265 & .008004 \\
\hline ardmore_cp d & .0263807 & .047877 & 0.55 & 0.592 & -.0779344 & .1306958 \\
\hline ardmore nd p & -5.012887 & 16.92836 & -0.30 & 0.772 & -41.89662 & 31.87085 \\
\hline ardmore_nd d & -.2582181 & 1.548699 & -0.17 & 0.870 & -3.632543 & 3.116107 \\
\hline cons & -55.57694 & 270.6126 & -0.21 & 0.841 & -645.1912 & 534.0373 \\
\hline
\end{tabular}

Windsor, IL

Linear regression

$\begin{array}{llr}\text { Number of obs } & & 22 \\ \text { F }(9, \quad 12) & 1.78 \\ \text { Prob }>\text { F } & = & 0.1728 \\ \text { R-squared } & = & 0.3938 \\ \text { Root MSE } & = & 8.7935\end{array}$

\begin{tabular}{|c|c|c|c|c|c|c|}
\hline windsoril_ $\sim$ s & Coef. & $\begin{array}{l}\text { Robust } \mathrm{HC} 3 \\
\text { Std. Err. }\end{array}$ & $t$ & $P>|t|$ & [ $95 \%$ & Interval] \\
\hline windsoril_ i & 489.3214 & 1518.145 & 0.32 & 0.753 & -2818.433 & 3797.07 \\
\hline windsor_cp & -9.07136 & 28.87484 & -0.31 & 0.759 & -71.98423 & 53.84151 \\
\hline windsor_gdd & -.3964352 & .4818188 & -0.82 & 0.427 & -1.446228 & .6533578 \\
\hline windsor_nd 2 & -342.4699 & 904.7362 & -0.38 & 0.712 & -2313.721 & 1628.781 \\
\hline windsor_cp2 & .3208783 & .2948763 & 1.09 & 0.298 & -.3216019 & .9633586 \\
\hline windsor_gdd2 & -.0000229 & .000221 & -0.10 & 0.919 & -.0005044 & .0004585 \\
\hline windsor_cp d & -.0089765 & .0104051 & -0.86 & 0.405 & -.0316473 & .0136943 \\
\hline windsor_nd $\sim p$ & 2.135824 & 28.39294 & 0.08 & 0.941 & -59.72707 & 63.99872 \\
\hline windsor_nd d & .6236645 & .488207 & 1.28 & 0.226 & -.4400472 & 1.687376 \\
\hline _cons & -77.10993 & 722.1689 & -0.11 & 0.917 & -1650.581 & 1496.361 \\
\hline
\end{tabular}

White Hall, IL

Linear regression

$\begin{array}{llr}\text { Number of obs } & = & 22 \\ \text { F } 7, \quad 13) & . \\ \text { Prob }>\text { F } & = & . \\ \text { R-squared } & = & 0.3811 \\ \text { Root MSE } & = & 9.015\end{array}$

\begin{tabular}{|c|c|c|c|c|c|c|}
\hline whitehalli s & Coef. & $\begin{array}{c}\text { Robust } \mathrm{HC} 3 \\
\text { Std. Err. }\end{array}$ & t & $P>|t|$ & [95\% Conf. & Inte \\
\hline hitehalli i & 1434.19 & 2443.882 & 0.59 & 0.567 & -3845.497 & 6713.877 \\
\hline whitehall_cp & -10.90035 & 19.24111 & -0.57 & 0.581 & -52.46825 & 30.66755 \\
\hline whiteha _odd & -20.14654 & 26.92923 & -0.75 & 0.468 & -78.32361 & 38.03053 \\
\hline hall i2 & $-1010 \cdot 714$ & 1677.731 & -0.60 & 0.557 & -4635.232 & 2613.804 \\
\hline whitehall p2 & .0812905 & .1187563 & 0.68 & 0.506 & -.1752669 & .3378479 \\
\hline whitehall d2 & .0134283 & .0179721 & 0.75 & 0.468 & -.025398 & .0522547 \\
\hline whiteha pgdd & 1.172964 & 1.568398 & 0.75 & 0.468 & -2.215354 & 4.561283 \\
\hline whitehal icp & 9.718563 & 23.16347 & 0.42 & 0.682 & -40.32307 & 59.76019 \\
\hline $\begin{array}{l}\text { lgad } \\
\text { cons }\end{array}$ & $\begin{array}{l}\text { (dropped) } \\
-419.0673\end{array}$ & 883.2496 & -0.47 & 0.643 & -2327.212 & 1489.078 \\
\hline
\end{tabular}


Beaverdam, KY

Linear regression

$\begin{array}{llr}\text { Number of obs } & & 22 \\ \text { F }(9, \quad 12) & 1.62 \\ \text { Prob }>\text { F } & = & 0.2135 \\ \text { R-squared } & = & 0.5779 \\ \text { Root MSE } & = & 8.8474\end{array}$

\begin{tabular}{|c|c|c|c|c|c|c|}
\hline eaverdamk & Coef. & Robust $\mathrm{HC} 3$ & $t$ & $>t+1$ & $95 \%$ & Tnterva \\
\hline & & & & & & \\
\hline eaverdamk i & -210.5991 & 6357.255 & -0.03 & 0.974 & -14061.87 & 13640.67 \\
\hline eaverdam_cp & 36.83333 & 50.50132 & 0.73 & 0.480 & -73.19958 & 146.8663 \\
\hline eaverd $\bar{g}$ dd & -.3199919 & 2.41394 & -0.13 & 0.897 & -5.579516 & 4.939532 \\
\hline beaverdam $\sim i 2$ & 577.565 & 4557.035 & 0.13 & 0.901 & -9351.362 & 10506.49 \\
\hline eaverdam p2 & -.037454 & .3603674 & -0.10 & 0.919 & -.8226272 & .7477191 \\
\hline dam d2 & .000176 & .0006753 & 0.26 & 0.799 & -.0012952 & .0016473 \\
\hline pgdd & -.0022333 & .033208 & -0.07 & 0.947 & -.0745873 & .0701208 \\
\hline eaverda icp & -47.72983 & 54.94607 & -0.87 & 0.402 & -167.447 & 71.98738 \\
\hline eaverd igdd & .2527671 & 2.280562 & 0.11 & 0.914 & -4.71615 & 5.221684 \\
\hline cons & -95.60423 & 2236.445 & -0.04 & 0.967 & -4968.4 & 4777.191 \\
\hline
\end{tabular}

Providence, KY

Linear regression

$\begin{array}{llr}\text { Number of obs } & & 22 \\ \mathrm{~F}(9, \quad 12) & =0.82 \\ \text { Prob }>\text { F } & = & 0.6132 \\ \text { R-squared } & =0.5393 \\ \text { Root MSE } & = & 10.495\end{array}$

\begin{tabular}{|c|c|c|c|c|c|c|}
\hline providence s & Coef. & $\begin{array}{l}\text { Robust } \mathrm{HC} 3 \\
\text { Std. Err. }\end{array}$ & $t$ & $P>|t|$ & [ $95 \%$ Conf. & Inte \\
\hline providence i & -1061.833 & 9404.04 & -0.11 & 0.912 & -21551.48 & 19427.81 \\
\hline providence p & 52.10165 & 92.13647 & 0.57 & 0.582 & -148.6465 & 252.8498 \\
\hline providn _ gdd & -.7556605 & 1.032285 & -0.73 & 0.478 & -3.004816 & 1.493495 \\
\hline providnec $\sim$ i2 & 1190.071 & 5704.588 & 0.21 & 0.838 & -11239.16 & 13619.3 \\
\hline providnec $\sim$ p2 & .1719209 & .6456064 & 0.27 & 0.795 & -1.234735 & 1.578576 \\
\hline providnec $\sim \mathrm{d} 2$ & .0001144 & .0001103 & 1.04 & 0.320 & -.0001259 & .0003548 \\
\hline providn pgdd & -.0004132 & .0075578 & -0.05 & 0.957 & -.0168801 & .0160538 \\
\hline providnece p & -75.17089 & 126.6589 & -0.59 & 0.564 & -351.1369 & 200.7952 \\
\hline providn igdd & .8964586 & 1.353336 & 0.66 & 0.520 & -2.052208 & 3.845125 \\
\hline _cons & 213.1955 & 3959.4 & 0.05 & 0.958 & -8413.596 & 8839.987 \\
\hline
\end{tabular}

Farmville, VA

Linear regression

$\begin{array}{lrr}\text { Number of obs } & & 20 \\ \text { F }(9, \quad 10) & 1.06 \\ \text { Prob }>\text { F } & =0.4585 \\ \text { R-squared } & =0.5876 \\ \text { Root MSE } & =9.7164\end{array}$

\begin{tabular}{|c|c|c|c|c|c|c|}
\hline farmvillev s & Coef. & $\begin{array}{c}\text { Robust } \mathrm{HC} 3 \\
\text { Std. Err. }\end{array}$ & t & $P>|t|$ & {$[95 \%$} & Inte \\
\hline farmvillev i & 3794.078 & 6525.373 & 0.58 & 0.574 & -10745.36 & 18333.51 \\
\hline farmville_cp & -104.5763 & 65.11351 & -1.61 & 0.139 & -249.6582 & 40.50564 \\
\hline farmvil gdd & -1.432604 & 1.957256 & -0.73 & 0.481 & -5.793641 & 2.928434 \\
\hline farmville i2 & -3496.564 & 4492.829 & -0.78 & 0.454 & -13507.21 & 6514.083 \\
\hline farmville p2 & .1088195 & .2101744 & 0.52 & 0.616 & -.3594783 & .5771174 \\
\hline farmville d2 & -.0002216 & .0012323 & -0.18 & 0.861 & -.0029673 & .0025242 \\
\hline farmvil pgdd & -.0161437 & .0340928 & -0.47 & 0.646 & -.0921071 & .0598198 \\
\hline farmvill icp & 127.93 & 79.83243 & 1.60 & 0.140 & -49.94769 & 305.8078 \\
\hline farmvil igdd & 2.05335 & 2.051961 & 1.00 & 0.341 & -2.518703 & 6.625404 \\
\hline _cons & -739.8238 & 2443.782 & -0.30 & 0.768 & -6184.91 & 4705.263 \\
\hline
\end{tabular}


Heppner, OR

Linear regression

$\begin{array}{llr}\text { Number of obs } & & 22 \\ \text { F }(9,12) & 8.77 \\ \text { Prob }>\text { F } & = & 0.0005 \\ \text { R-squared } & = & 0.5673 \\ \text { Root MSE } & = & 11.331\end{array}$

\begin{tabular}{|c|c|c|c|c|c|c|}
\hline heppneror_ s & Coef. & $\begin{array}{c}\text { Robust } \mathrm{HC} 3 \\
\text { Std. Err. }\end{array}$ & $t$ & $P>|t|$ & [95\% Conf. & Interval] \\
\hline heppneror_i & 1175.42 & 1330.759 & 0.88 & 0.394 & -1724.055 & 4074.894 \\
\hline heppner_cp & 18.71314 & 39.68772 & 0.47 & 0.646 & -67.75897 & 105.1852 \\
\hline heppner_ḡdd & -3.462206 & 2.789068 & -1.24 & 0.238 & -9.539062 & 2.614651 \\
\hline heppner_nd $\sim 2$ & -1349.327 & 1820.77 & -0.74 & 0.473 & -5316.444 & 2617.79 \\
\hline heppners_cp2 & -1.926163 & 1.97864 & -0.97 & 0.350 & -6.237249 & 2.384924 \\
\hline heppner_ōgdd2 & .0466862 & .0240823 & 1.94 & 0.076 & -.0057847 & .099157 \\
\hline heppner_cp d & .1729583 & .2009066 & 0.86 & 0.406 & -.2647795 & .6106961 \\
\hline heppner_nd p & -5.296972 & 94.78628 & -0.06 & 0.956 & -211.8185 & 201.2246 \\
\hline heppner_nd d & 3.139582 & 6.438356 & 0.49 & 0.635 & -10.88839 & 17.16755 \\
\hline _cons & -229.9842 & 252.8717 & -0.91 & 0.381 & -780.9444 & 320.976 \\
\hline
\end{tabular}

\section{Eltopia, WA}

Linear regression

$\begin{array}{llr}\text { Number of obs } & = & 22 \\ \mathrm{~F}(9, \quad 12) & =0.40 \\ \text { Prob }>\text { F } & = & 0.9141 \\ \text { R-squared } & =0.1886 \\ \text { Root MSE } & =9.4417\end{array}$

\begin{tabular}{|c|c|c|c|c|c|c|}
\hline & & Robust $\mathrm{HC} 3$ & & & & \\
\hline eltopiawa_ s & Coef. & Std. Err. & $t$ & $P>|t|$ & [95\% Conf. & Inte \\
\hline eltopiawa_i & 1344.134 & 3414.137 & 0.39 & 0.701 & -6094.631 & 8782.899 \\
\hline eltopia_cp & -150.3141 & 289.7823 & -0.52 & 0.613 & -781.6956 & 481.0674 \\
\hline eltopia_ḡdd & 1.359736 & 1.482075 & 0.92 & 0.377 & -1.869428 & 4.588901 \\
\hline eltopia_ñd 2 & -1115.703 & 2749.532 & -0.41 & 0.692 & -7106.419 & 4875.012 \\
\hline eltopiā_cp2 & -9.439926 & 13.51372 & -0.70 & 0.498 & -38.8838 & 20.00395 \\
\hline eltopia_ḡdd2 & .0005112 & .0020714 & 0.25 & 0.809 & -.0040021 & .0050245 \\
\hline eltopia_cp d & .0731391 & .2220345 & 0.33 & 0.748 & -.4106326 & .5569108 \\
\hline eltopia_nd p & 255.2606 & 454.7717 & 0.56 & 0.585 & -735.6018 & 1246.123 \\
\hline eltopia_nd d & -2.230116 & 2.539788 & -0.88 & 0.397 & -7.763838 & 3.303606 \\
\hline _cons & -356.6675 & 1083.387 & -0.33 & 0.748 & -2717.165 & 2003.83 \\
\hline
\end{tabular}

\section{Menomonie, WI}

Linear regression

$\begin{array}{llr}\text { Number of obs } & & 18 \\ \text { F }(9, \quad 8) & =1.17 \\ \text { Prob }>\text { F } & =0.4157 \\ \text { R-squared } & = & 0.7344 \\ \text { Root MSE } & = & 5.3801\end{array}$

\begin{tabular}{|c|c|c|c|c|c|c|}
\hline menomoniew $\sim \mathrm{s}$ & Coef. & $\begin{array}{l}\text { Robust HC3 } \\
\text { Std. Err. }\end{array}$ & t & $P>|t|$ & [95\% Conf. & Interval ] \\
\hline menomoniew $\sim$ i & 11344.66 & 9391.079 & 1.21 & 0.262 & -10311.21 & 33000.52 \\
\hline menomonie_cp & -39.93709 & 54.52757 & -0.73 & 0.485 & -165.6779 & 85.80371 \\
\hline menomon gdd & -1.144775 & 1.621529 & -0.71 & 0.500 & -4.884027 & 2.594477 \\
\hline menomonie i2 & -7620.355 & 5916.175 & -1.29 & 0.234 & -21263.08 & 6022.369 \\
\hline menomonie p2 & .4407006 & .3541906 & 1.24 & 0.249 & -.3760643 & 1.257466 \\
\hline menomonie d2 & .0005281 & .0003165 & 1.67 & 0.134 & -.0002016 & .0012579 \\
\hline menomon $\sim$ pgdd & .0395373 & .0211339 & 1.87 & 0.098 & -.0091976 & .0882722 \\
\hline menomoni icp & 29.12969 & 58.79096 & 0.50 & 0.634 & -106.4425 & 164.7019 \\
\hline menomon igdd & .5881746 & 1.997048 & 0.29 & 0.776 & -4.017025 & 5.193374 \\
\hline _cons & -4011.876 & 3735.6 & -1.07 & 0.314 & -12626.19 & 4602.434 \\
\hline
\end{tabular}


Arlington, WI

Linear regression

$\begin{array}{llr}\text { Number of obs } & & 22 \\ \text { F }(9,12) & 0.72 \\ \text { Prob }>\text { F } & = & 0.6874 \\ \text { R-squared } & =0.5418 \\ \text { Root MSE } & =9.9203\end{array}$

\begin{tabular}{|c|c|c|c|c|c|c|}
\hline arlingtonw s & Coef. & $\begin{array}{l}\text { Robust } \mathrm{HC} 3 \\
\text { Std. Err. }\end{array}$ & $t$ & $P>|t|$ & [95\% Conf. & Inte \\
\hline lingtonw $\sim$ & 6093.416 & 3393.856 & 1.80 & 0.098 & -1301.16 & 13487.99 \\
\hline lington_cp & -5.121173 & 69.05195 & -0.07 & 0.942 & -155.5724 & 145.3301 \\
\hline slingt $\bar{g}$ dd & .4694262 & 2.304255 & 0.20 & 0.842 & -4.551115 & 5.489967 \\
\hline lington $\sim$ i2 & -4027.817 & 2304.19 & -1.75 & 0.106 & -9048.215 & 992.5821 \\
\hline lington $\sim \mathrm{p} 2$ & -.2857965 & .5275149 & -0.54 & 0.598 & -1.435153 & .8635598 \\
\hline lington $\mathrm{d} 2$ & .0006148 & .0012177 & 0.50 & 0.623 & -.0020385 & .003268 \\
\hline lingt $\sim$ pgdd & .0213703 & .0568383 & 0.38 & 0.713 & -.1024697 & .1452104 \\
\hline lingto icp & 16.41859 & 69.63959 & 0.24 & 0.818 & -135.313 & 168.1502 \\
\hline ngt igdd & -1.251544 & 2.203906 & -0.57 & 0.581 & -6.053442 & 3.550353 \\
\hline cons & -2278.425 & 1403.472 & -1.62 & 0.130 & -5336.328 & 779.4778 \\
\hline
\end{tabular}

\section{Sellingsgrove, PA}

Linear regression

$\begin{array}{lrr}\text { Number of obs } & & 22 \\ \mathrm{~F}(5, \quad 16) & = & 1.51 \\ \text { Prob }>\text { F } & = & 0.2407 \\ \text { R-squared } & = & 0.2868 \\ \text { Root MSE } & = & 5.6887\end{array}$

\begin{tabular}{|c|c|c|c|c|c|c|}
\hline sellingsgr s & Coef. & $\begin{array}{l}\text { Robust HC3 } \\
\text { Std. Err. }\end{array}$ & $t$ & $P>|t|$ & [95\% Conf. & Interval] \\
\hline $\begin{array}{l}\text { selingsgro i } \\
\text { selingsgro p } \\
\text { selling _gdd }\end{array}$ & $\begin{array}{l}-856.9242 \\
-12.96831 \\
\text { (dropped) }\end{array}$ & $\begin{array}{l}2659.073 \\
21.48268\end{array}$ & $\begin{array}{l}-0.32 \\
-0.60\end{array}$ & $\begin{array}{l}0.751 \\
0.555\end{array}$ & $\begin{array}{l}-6493.907 \\
-58.50955\end{array}$ & $\begin{array}{l}4780.059 \\
32.57294\end{array}$ \\
\hline sellingsḡ $\sim 2$ & 377.883 & 1931.777 & 0.20 & 0.847 & -3717.301 & 4473.067 \\
\hline $\begin{array}{l}\text { sellingsg p2 } \\
\text { sellingsg d2 } \\
\text { selling pgdd }\end{array}$ & $\begin{array}{r}.0164978 \\
\text { (dropped) } \\
\text { (dropped) }\end{array}$ & 412 & 0.09 & 0.929 & -.3721682 & .4051638 \\
\hline $\begin{array}{l}\text { sellingsgr p } \\
\text { selling igdd }\end{array}$ & $\begin{array}{r}15.35944 \\
\text { (dropped) }\end{array}$ & 26.57497 & 0.58 & 0.571 & -40.97697 & 71.69586 \\
\hline cons & 486.0191 & 914.7494 & 0.53 & 0.603 & -1453.163 & 2425.201 \\
\hline
\end{tabular}




\section{Regression Results for Hay Yields}

\section{Algona, IA}

Linear regression

$\begin{array}{llr}\text { Number of obs } & & 22 \\ \text { F }(9, \quad 12) & 1.89 \\ \text { Prob }>\text { F } & = & 0.1504 \\ \text { R-squared } & = & 0.7371 \\ \text { Root MSE } & = & .41579\end{array}$

\begin{tabular}{|c|c|c|c|c|c|c|}
\hline algonaia_y s & Coef. & $\begin{array}{l}\text { Robust HC3 } \\
\text { Std. Err. }\end{array}$ & $t$ & $P>|t|$ & [ $95 \%$ Conf. & Interval] \\
\hline Igonaia_m i & 262.3999 & 669.7544 & 0.39 & 0.702 & -1196.87 & 1721.669 \\
\hline algonā_cp & .5999193 & 4.172153 & 0.14 & 0.888 & -8.490422 & 9.69026 \\
\hline algona_gdd & -.1122503 & .3040673 & -0.37 & 0.718 & -.774756 & 553 \\
\hline longona_m 2 & -158.3539 & 416.588 & -0.38 & 0.710 & -1066.021 & 749.3133 \\
\hline algona_cp2 & -.0106205 & .0131742 & -0.81 & 0.436 & -.0393247 & .0180837 \\
\hline algona_gdd2 & .0000495 & .0000792 & 0.63 & 0.544 & -.000123 & .000222 \\
\hline algona_cpgdd & $-5.98 e-06$ & .0018088 & -0.00 & 0.997 & -.003947 & .003935 \\
\hline lgona_ndv $\sim p$ & -.1827512 & 4.95729 & -0.04 & 0.971 & -10.98376 & 10.61826 \\
\hline lgona_ndv d & .1253855 & .3469944 & 0.36 & 0.724 & -.6306503 & .8814213 \\
\hline _cons & -109.2842 & 271.876 & -0.40 & 0.695 & -701.6512 & 483.0827 \\
\hline
\end{tabular}

\section{Rockrapids, IA}

Linear regression

$\begin{array}{llr}\text { Number of obs } & & 22 \\ \mathrm{~F}(5, \quad 16) & =1.08 \\ \text { Prob }>\mathrm{F} & = & 0.4086 \\ \text { R-squared } & = & 0.3108 \\ \text { Root MSE } & = & .56302\end{array}$

\begin{tabular}{|c|c|c|c|c|c|c|}
\hline rockrapids s & Coef. & $\begin{array}{l}\text { Robust } \mathrm{HC} 3 \\
\text { Std. Err. }\end{array}$ & $t$ & $P>|t|$ & [95\% Conf. & Interval] \\
\hline ockrapids i & 601.8646 & 440.198 & 1.37 & 0.190 & -331.3135 & 1535.043 \\
\hline $\begin{array}{l}\text { ockrapi _cp } \\
\text { ockrap gdd }\end{array}$ & $\begin{array}{r}1.367937 \\
\text { (dropped) }\end{array}$ & 1.519516 & 0.90 & 0.381 & -1.853294 & 4.589168 \\
\hline ckrapid $\sim 2$ & -333.9187 & 251.1709 & -1.33 & 0.202 & -866.3772 & 198.5398 \\
\hline $\begin{array}{l}\text { rockrapid p2 } \\
\text { rockrapid d2 } \\
\text { rockrap pgdd }\end{array}$ & $\begin{array}{l}.0062002 \\
\text { (dropped) } \\
\text { (dropped) }\end{array}$ & .01451 & 0.43 & 0.675 & -.0245596 & .03696 \\
\hline $\begin{array}{l}\text { ockrapi icp } \\
\text { ockrap igdd }\end{array}$ & $\begin{array}{l}-1.771068 \\
\text { (dropped) }\end{array}$ & 1.633952 & -1.08 & 0.294 & -5.234892 & 1.692755 \\
\hline cons & -265.6537 & 191.6626 & -1.39 & 0.185 & -671.9602 & 140.6528 \\
\hline
\end{tabular}


Saluda, SC

Linear regression

$\begin{array}{llr}\text { Number of obs } & = & 22 \\ \text { F }(8, \text { 13) } & 849.27 \\ \text { Prob }>\text { F } & =0.0000 \\ \text { R-squared } & =0.5821 \\ \text { Root MSE } & = & .39648\end{array}$

\begin{tabular}{|c|c|c|c|c|c|c|}
\hline saluda_yie s & Coef. & $\begin{array}{c}\text { Robust } \mathrm{HC} 3 \\
\text { Std. Err. }\end{array}$ & $t$ & $P>|t|$ & [ $95 \%$ Conf. & Interval \\
\hline saludasc_m i & -341.6133 & 762.1852 & -0.45 & 0.661 & -1988.214 & 1304.988 \\
\hline saluda_cp & -.7317439 & 2.504153 & -0.29 & 0.775 & -6.141637 & 4.678149 \\
\hline saluda_ḡgdd & .2621284 & .4768213 & 0.55 & 0.592 & -.7679814 & 1.292238 \\
\hline saluda_ndvi2 & 214.2217 & 474.6045 & 0.45 & 0.659 & -811.099 & 1239.542 \\
\hline salud̄a_cp2 & .0014702 & .0110402 & 0.13 & 0.896 & -.0223807 & .0253212 \\
\hline saluda_gdd2 & .0133031 & .0130254 & 1.02 & 0.326 & -.0148366 & .0414428 \\
\hline saluda_cpgdd & -.0312691 & .0389084 & -0.80 & 0.436 & -.1153256 & .0527874 \\
\hline $\begin{array}{l}\text { saluda_ndv } \sim \text { p } \\
\text { saluda ndv } \sim d\end{array}$ & $\begin{array}{r}.9233399 \\
\text { (dropped) }\end{array}$ & 3.168726 & 0.29 & 0.775 & -5.922277 & 7.768957 \\
\hline cons & 138.2707 & 305.0668 & 0.45 & 0.658 & -520.7861 & 797.3275 \\
\hline
\end{tabular}

\section{Angelica, NY}

Linear regression

$\begin{array}{llr}\text { Number of obs } & & 21 \\ \mathrm{~F}(8, \mathrm{8}, 12) & 9.12 \\ \text { Prob }>\text { F } & =0.0005 \\ \text { R-squared } & =0.2632 \\ \text { Root MSE } & = & .19965\end{array}$

\begin{tabular}{|c|c|c|c|c|c|c|}
\hline angelicany s & Coef. & $\begin{array}{l}\text { Robust } \mathrm{HC} 3 \\
\text { Std. Err. }\end{array}$ & $t$ & $P>|t|$ & [ $95 \%$ Conf. & Interval] \\
\hline angelicany i & -20.27773 & 107.8681 & -0.19 & 0.854 & -255.3022 & 214.7468 \\
\hline angelica_cp & .2140865 & .74112 & 0.29 & 0.778 & -1.400675 & 1.828848 \\
\hline angelica_ḡdd & .0602444 & .0889176 & 0.68 & 0.511 & -.1334905 & .2539792 \\
\hline angelica_n 2 & 13.11143 & 66.05102 & 0.20 & 0.846 & -130.8014 & 157.0242 \\
\hline angelica_cp2 & -.0041946 & .0074905 & -0.56 & 0.586 & -.0205151 & .0121258 \\
\hline angelica_g 2 & -.0013496 & .0012396 & -1.09 & 0.298 & -.0040504 & .0013512 \\
\hline angelica_c d & -.000628 & .0058612 & -0.11 & 0.916 & -.0133984 & .0121424 \\
\hline $\begin{array}{l}\text { angelica_n p } \\
\text { angelica } n \sim d\end{array}$ & $\begin{array}{l}-.1043994 \\
\text { (dropped) }\end{array}$ & 1.007592 & -0.10 & 0.919 & -2.299753 & 2.090954 \\
\hline cons & 8.90666 & 44.86055 & 0.20 & 0.846 & -88.83607 & 106.6494 \\
\hline
\end{tabular}

\section{Riverhead, NY}

Linear regression

$\begin{array}{lrr}\text { Number of obs } & & 21 \\ \text { F }(9, \quad 11) & 0.70 \\ \text { Prob }>\text { F } & =06975 \\ \text { R-squared } & =0.2308 \\ \text { Root MSE } & = & .53072\end{array}$

\begin{tabular}{|c|c|c|c|c|c|c|}
\hline riverheadn s & Coef. & $\begin{array}{c}\text { Robust HC3 } \\
\text { Std. Err. }\end{array}$ & t & $P>|t|$ & [ $95 \%$ Conf. & Interval ] \\
\hline riverheadn i & -64.86146 & 54.99914 & -1.18 & 0.263 & -185.9138 & 56.19084 \\
\hline riverhead_cp & -.6891965 & .551371 & -1.25 & 0.237 & -1.902756 & .524363 \\
\hline riverhe gdd & -.020673 & .0475026 & -0.44 & 0.672 & -.1252255 & .0838795 \\
\hline riverhead i2 & 43.55851 & 58.28963 & 0.75 & 0.471 & -84.73611 & 171.8531 \\
\hline riverhead p2 & .0030419 & .0058801 & 0.52 & 0.615 & -.0099001 & .0159839 \\
\hline riverhead d2 & $-1.84 e-07$ & .0000741 & -0.00 & 0.998 & -.0001634 & .000163 \\
\hline riverhe pgdd & .000542 & .0021827 & 0.25 & 0.808 & -.0042621 & .0053461 \\
\hline riverhea icp & 1.759066 & 1.187307 & 1.48 & 0.167 & -.85418 & 4.372311 \\
\hline riverhe igdd & .048378 & .0695806 & 0.70 & 0.501 & -.1047678 & .2015238 \\
\hline _cons & 19.01838 & 1681 & 1.38 & 0.196 & -11.39221 & 49.42898 \\
\hline
\end{tabular}


Fredonia, NY

Linear regression

$\begin{array}{lrr}\text { Number of obs } & & 21 \\ \text { F }(9, \quad 11) & 0.38 \\ \text { Prob }>\text { F } & =0.9226 \\ \text { R-squared } & =0.3827 \\ \text { Root MSE } & = & .22018\end{array}$

\begin{tabular}{|c|c|c|c|c|c|c|}
\hline fredoniany s & Coef. & $\begin{array}{c}\text { Robust } \mathrm{HC} 3 \\
\text { Std. Err. }\end{array}$ & $t$ & $P>|t|$ & [95\% Conf. & Interval] \\
\hline fredoniany i & -.5535508 & 9.360303 & -0.06 & 0.954 & -21.15544 & 20.04834 \\
\hline fredonia_cp & .0178996 & .2949693 & 0.06 & 0.953 & -.6313234 & .6671226 \\
\hline fredonia_odd & .0103718 & .0261205 & 0.40 & 0.699 & -.0471192 & .0678627 \\
\hline fredonia_n $\sim 2$ & 2.412512 & 6.037043 & 0.40 & 0.697 & -10.87493 & 15.69995 \\
\hline fredonia-cp2 & .0008606 & .0092877 & 0.09 & 0.928 & -.0195814 & .0213027 \\
\hline fredonia_g 2 & -.0000333 & .0000627 & -0.53 & 0.607 & -.0001713 & .0001048 \\
\hline fredonia_c $\sim$ d & .0000355 & .001338 & 0.03 & 0.979 & -.0029094 & .0029803 \\
\hline fredonia-n $n$ & -.0540006 & .3631268 & -0.15 & 0.884 & -.8532374 & .7452361 \\
\hline fredonia-n d & -.0108836 & .0196451 & -0.55 & 0.591 & -.0541222 & .0323551 \\
\hline _cons & 1.702421 & 4.143479 & 0.41 & 0.689 & -7.417315 & 10.82216 \\
\hline
\end{tabular}

\section{Du Quoin, IL}

Linear regression

$\begin{array}{llr}\text { Number of obs } & & 22 \\ \text { F }(9, \quad 12) & =1.03 \\ \text { Prob }>\text { F } & =0.4694 \\ \text { R-squared } & =0.4752 \\ \text { Root MSE } & =.3372\end{array}$

\begin{tabular}{|c|c|c|c|c|c|c|}
\hline duquoinil_ s & Coef. & $\begin{array}{l}\text { Robust } \mathrm{HC} 3 \\
\text { Std. Err. }\end{array}$ & $t$ & $P>|t|$ & [ $95 \%$ Conf. & Interval] \\
\hline duquoinil_ i & -18.67534 & 52.93353 & -0.35 & 0.730 & -134.0076 & 96.6569 \\
\hline duquoin_cp & -.5137276 & .6642261 & -0.77 & 0.454 & -1.960952 & .9334968 \\
\hline duquoin_ḡdd & -.0151936 & .1695005 & -0.09 & 0.930 & -.3845034 & .3541161 \\
\hline duquoin_nd 2 & 6.589409 & 30.22375 & 0.22 & 0.831 & -59.26248 & 72.4413 \\
\hline duquoin_cp2 & .0007281 & .0038709 & 0.19 & 0.854 & -.0077058 & .0091621 \\
\hline duquoin_ḡgdd2 & -.0001593 & .0003687 & -0.43 & 0.673 & -.0009626 & .000644 \\
\hline duquoin_cp d & .0003412 & .0014412 & 0.24 & 0.817 & -.0027988 & .0034812 \\
\hline duquoin_nd $\sim p$ & .7010807 & .7776018 & 0.90 & 0.385 & -.9931681 & 2.39533 \\
\hline duquoin_nd d & .0204497 & .2108346 & 0.10 & 0.924 & -.4389193 & .4798188 \\
\hline _cons & 12.54916 & 23.60058 & 0.53 & 0.605 & -38.87208 & 63.9704 \\
\hline
\end{tabular}

\section{Minonk, IL}

Linear regression

$\begin{array}{lrr}\text { Number of obs } & & 17 \\ \text { F }(8, \quad 8) & =0.66 \\ \text { Prob }>\text { F } & =0.7180 \\ \text { R-squared } & = & 0.4792 \\ \text { Root MSE } & = & .79376\end{array}$

\begin{tabular}{|c|c|c|c|c|c|c|}
\hline minonkil_y s & Coef. & $\begin{array}{c}\text { Robust } \mathrm{HC} 3 \\
\text { Std. Err. }\end{array}$ & t & $P>|t|$ & [95\% Conf. & Interval] \\
\hline minonkil_m i & 243.1912 & 288.6595 & 0.84 & 0.424 & -422.4587 & 908.8411 \\
\hline minons̄_cp & -1.926516 & 4.576832 & -0.42 & 0.685 & -12.48071 & 8.627678 \\
\hline minonk_ōgdd & .3390017 & .7044986 & 0.48 & 0.643 & -1.285575 & 1.963578 \\
\hline minonk_ndvi2 & -167.5637 & 197.1238 & -0.85 & 0.420 & -622.1321 & 287.0047 \\
\hline minonk_cp2 & -.0201686 & .0171822 & -1.17 & 0.274 & -.0597909 & .0194537 \\
\hline minonk_gdd2 & -.0447596 & .4187465 & -0.11 & 0.918 & -1.010391 & .9208716 \\
\hline minonk_cpgdd & -.0212073 & .1116171 & -0.19 & 0.854 & -.2785967 & .2361821 \\
\hline minonk_ndv $\sim p$ & 2.988087 & 5.195593 & 0.58 & 0.581 & -8.992972 & 14.96915 \\
\hline $\begin{array}{r}\text { nav d } \\
\text { cons }\end{array}$ & $\begin{array}{l}\text { (dropped) } \\
-86.27609\end{array}$ & 105.6009 & -0.82 & 0.438 & -329.7922 & 157.24 \\
\hline
\end{tabular}


Windsor, IL

Linear regression

$\begin{array}{llr}\text { Number of obs } & & 22 \\ \text { F }(9,12) & 0.94 \\ \text { Prob }>\text { F } & = & 0.5240 \\ \text { R-squared } & = & 0.4874 \\ \text { Root MSE } & = & .52194\end{array}$

\begin{tabular}{|c|c|c|c|c|c|c|}
\hline windsoril_ s & Coef. & $\begin{array}{c}\text { Robust } \mathrm{HC} 3 \\
\text { Std. Err. }\end{array}$ & $t$ & $P>|t|$ & [ $95 \%$ Conf. & Interval] \\
\hline windsoril_i & -64.93517 & 98.31755 & -0.66 & 0.521 & -279.1507 & 149.2804 \\
\hline windsor $\mathrm{cp}$ & 1.286248 & 1.222473 & 1.05 & 0.313 & -1.377293 & 3.949789 \\
\hline windsor_ḡdd & -.0036794 & .043512 & -0.08 & 0.934 & -.098484 & .0911252 \\
\hline windsor nd 2 & 47.94735 & 62.01857 & 0.77 & 0.454 & -87.1795 & 183.0742 \\
\hline windsor_cp2 & -.0091679 & .0153632 & -0.60 & 0.562 & -.0426414 & .0243057 \\
\hline windsor ḡdd2 & $-2.32 e-06$ & .0000247 & -0.09 & 0.927 & -.0000561 & .0000515 \\
\hline windsor cp d & -.0001545 & .0007784 & -0.20 & 0.846 & -.0018504 & .0015414 \\
\hline windsor nd p & -1.193685 & 1.039095 & -1.15 & 0.273 & -3.457678 & 1.070308 \\
\hline windsor_nd d & .0093468 & .0460433 & 0.20 & 0.843 & -.090973 & .1096665 \\
\hline _cons & 21.30999 & 35.10377 & 0.61 & 0.555 & -55.17455 & 97.79454 \\
\hline
\end{tabular}

White Hall, IL

Linear regression

$\begin{array}{llr}\text { Number of obs } & & 22 \\ \mathrm{~F}(7, \quad 13) & = & . \\ \text { Prob }>\text { F } & = & . \\ \text { R-squared } & = & 0.7430 \\ \text { Root MSE } & = & .25657\end{array}$

\begin{tabular}{|c|c|c|c|c|c|c|}
\hline whitehalli s & Coef. & $\begin{array}{l}\text { Robust HC3 } \\
\text { Std. Err. }\end{array}$ & t & $P>|t|$ & [95\% Conf. & Interval ] \\
\hline halli i & 31.27656 & 51.24848 & 0.61 & 0.552 & -79.43906 & 141.9922 \\
\hline lall_cp & -.8226845 & .3460713 & -2.38 & 0.033 & -1.570326 & -.075043 \\
\hline whiteha _ōgdd & -1.101324 & 1.168371 & -0.94 & 0.363 & -3.625437 & 1.422788 \\
\hline whitehall i2 & -31.77828 & 34.13604 & -0.93 & 0.369 & -105.5247 & 41.96816 \\
\hline all p2 & -.0090593 & .0048293 & -1.88 & 0.083 & -.0194924 & .0013738 \\
\hline hall d2 & .000732 & .0007796 & 0.94 & 0.365 & -.0009523 & .0024163 \\
\hline hiteha pgdd & .0641959 & .0680612 & 0.94 & 0.363 & -.0828415 & .2112332 \\
\hline whitehal icp & 1.352033 & .4481148 & 3.02 & 0.010 & .3839395 & 2.320126 \\
\hline niteha igdd & (dropped) & & & & & \\
\hline _cons & -3.389193 & 19.20618 & -0.18 & 0.863 & -44.88162 & 38.10324 \\
\hline
\end{tabular}

\section{Beaverdam, KY}

Linear regression

$\begin{array}{llr}\text { Number of obs } & & 22 \\ \text { F }(9, \quad 12) & 1.38 \\ \text { Prob }>\text { F } & =0.2967 \\ \text { R-squared } & = & 0.6385 \\ \text { Root MSE } & = & .31252\end{array}$

\begin{tabular}{|c|c|c|c|c|c|c|}
\hline beaverdamk s & Coef. & $\begin{array}{l}\text { Robust HC3 } \\
\text { Std. Err. }\end{array}$ & t & $P>|t|$ & [95\% Conf. & val ] \\
\hline beaverdamk i & -32.04028 & 426.1315 & -0.08 & 0.941 & -960.501 & 896.4205 \\
\hline beaverdam_cp & -.2918078 & 3.475541 & -0.08 & 0.934 & -7.864361 & 7.280745 \\
\hline beaverd _gdd & -.0019621 & .0945299 & -0.02 & 0.984 & -.2079251 & .2040008 \\
\hline beaverdam i2 & 16.8392 & 269.6761 & 0.06 & 0.951 & -570.7346 & 604.413 \\
\hline beaverdam p2 & .0016308 & .0224166 & 0.07 & 0.943 & -.0472108 & .0504723 \\
\hline beaverdam $\sim \mathrm{d} 2$ & $-9.06 e-06$ & .0000239 & -0.38 & 0.712 & -.0000612 & .0000431 \\
\hline beaverd pgdd & -.0000769 & .0014751 & -0.05 & 0.959 & -.0032909 & .0031372 \\
\hline beaverda icp & .4245456 & 4.024037 & 0.11 & 0.918 & -8.343078 & 9.192169 \\
\hline beaverd igdd & .0098705 & .1035827 & 0.10 & 0.926 & -.2158167 & .2355578 \\
\hline _cons & 15.65402 & 172.0746 & 0.09 & 0.929 & -359.2644 & 390.5724 \\
\hline
\end{tabular}


Providence, KY

Linear regression

$\begin{array}{llr}\text { Number of obs } & & 22 \\ \text { F }(9,12) & 1.74 \\ \text { Prob }>\text { F } & = & 0.1840 \\ \text { R-squared } & =0.6093 \\ \text { Root MSE } & = & .23107\end{array}$

\begin{tabular}{|c|c|c|c|c|c|c|}
\hline providence s & Coef. & $\begin{array}{l}\text { Robust } \mathrm{HC} 3 \\
\text { Std. Err. }\end{array}$ & $t$ & $P>|t|$ & [ $95 \%$ Conf. & val] \\
\hline providence i & 102.5668 & 204.1666 & 0.50 & 0.625 & -342.2741 & 547.4077 \\
\hline providence $\sim$ p & 1.071016 & 1.756766 & 0.61 & 0.553 & -2.756649 & 4.898682 \\
\hline providn _gdd & .0138128 & .0257694 & 0.54 & 0.602 & -.0423339 & .0699594 \\
\hline providnec $\sim$ i2 & -50.24293 & 130.4747 & -0.39 & 0.707 & -334.5229 & 234.037 \\
\hline providnec $\sim$ p2 & .0016522 & .0096592 & 0.17 & 0.867 & -.0193935 & .0226979 \\
\hline providnec $\sim \mathrm{d} 2$ & $-8.43 e-07$ & $1.79 \mathrm{e}-06$ & -0.47 & 0.646 & $-4.74 e-06$ & $3.06 e-06$ \\
\hline providn pgdd & -.0000988 & .0001552 & -0.64 & 0.536 & -.000437 & .0002394 \\
\hline providnece p p & -1.359444 & 2.455217 & -0.55 & 0.590 & -6.708903 & 3.990015 \\
\hline providn igdd & -.0158313 & .0336536 & -0.47 & 0.646 & -.0891562 & .0574936 \\
\hline _cons & -47.58284 & 82.48739 & -0.58 & 0.575 & -227.3074 & 132.1417 \\
\hline
\end{tabular}

Farmville, VA

Linear regression

$\begin{array}{lrr}\text { Number of obs } & = & 10 \\ \mathrm{~F}(5, \quad 4) & =2.68 \\ \text { Prob }>\text { F } & = & 0.1802 \\ \text { R-squared } & = & 0.7594 \\ \text { Root MSE } & = & .25828\end{array}$

\begin{tabular}{|c|c|c|c|c|c|c|}
\hline farmvillev s & Coef. & $\begin{array}{l}\text { Robust } \mathrm{HC} 3 \\
\text { Std. Err. }\end{array}$ & $t$ & $P>|t|$ & [ $95 \%$ Conf. & Interval] \\
\hline farmvillev i & -378.277 & 283.1911 & -1.34 & 0.253 & -1164.541 & 407.9876 \\
\hline farmville_cp & 1.99739 & 3.562707 & 0.56 & 0.605 & -7.89427 & 11.88905 \\
\hline farmvil _ gdd & (dropped) & & & & & \\
\hline farmville $\sim i 2$ & 260.4004 & 178.1108 & 1.46 & 0.218 & -234.1144 & 754.9152 \\
\hline farmville p2 & -.0042363 & .0213135 & -0.20 & 0.852 & -.0634121 & .0549395 \\
\hline farmville d2 & (dropped) & & & & & \\
\hline farmvil pgdd & (dropped) & & & & & \\
\hline farmvill icp & -2.325044 & 3.867337 & -0.60 & 0.580 & -13.06249 & 8.412406 \\
\hline farmvil igdd & (dropped) & & & & & \\
\hline _cons & 136.8175 & 118.9477 & 1.15 & 0.314 & -193.4343 & 467.0693 \\
\hline
\end{tabular}

\section{Heppner, OR}

Linear regression

$\begin{array}{llr}\text { Number of obs } & & 21 \\ \text { F }(9,11) & 0.15 \\ \text { Prob }>\text { F } & = & 0.9954 \\ \text { R-squared } & =0.3950 \\ \text { Root MSE } & = & .58663\end{array}$

\begin{tabular}{|c|c|c|c|c|c|c|}
\hline heppneror_ s & Coef. & $\begin{array}{l}\text { Robust } \mathrm{HC} 3 \\
\text { Std. Err. }\end{array}$ & $t$ & $P>|t|$ & [95\% Conf. & Interval] \\
\hline heppneror_ i & -14.27929 & 142.7063 & -0.10 & 0.922 & -328.3737 & 299.815 \\
\hline heppner_cp & -.5829756 & 5.165 & -0.11 & 0.912 & -11.95106 & 10.78511 \\
\hline heppner_gdd & .2390269 & .7233623 & 0.33 & 0.747 & -1.353083 & 1.831137 \\
\hline heppner_nd 2 & 10.8174 & 168.7623 & 0.06 & 0.950 & -360.6259 & 382.2607 \\
\hline heppner_cp2 & -.023095 & .2858307 & -0.08 & 0.937 & -.6522042 & .6060142 \\
\hline heppner_gdd2 & -.0016311 & .0044104 & -0.37 & 0.719 & -.0113382 & .0080761 \\
\hline heppner_cp d & -.0191319 & .0302127 & -0.63 & 0.540 & -.0856296 & .0473657 \\
\hline heppner_nd $\sim$ p & 1.948754 & 11.64613 & 0.17 & 0.870 & -23.6842 & 27.58171 \\
\hline $\begin{array}{r}\text { heppner_nd d } \\
\text { cons }\end{array}$ & $\begin{array}{r}-.309111 \\
8.5916\end{array}$ & $\begin{array}{l}1.565155 \\
32.67451\end{array}$ & $\begin{array}{r}-0.20 \\
0.26\end{array}$ & $\begin{array}{l}0.847 \\
0.797\end{array}$ & $\begin{array}{r}-3.753993 \\
-63.3245\end{array}$ & $\begin{array}{r}3.135771 \\
80.5077\end{array}$ \\
\hline
\end{tabular}


Eltopia, WA

Linear regression

$\begin{array}{lrr}\text { Number of obs } & & 18 \\ \text { F }(9, \quad 8) & =0.84 \\ \text { Prob }>\text { F } & = & 0.6033 \\ \text { R-squared } & = & 0.6681 \\ \text { Root MSE } & = & .44806\end{array}$

\begin{tabular}{|c|c|c|c|c|c|c|}
\hline eltopiawa_ s & Coef. & $\begin{array}{c}\text { Robust } \mathrm{HC} 3 \\
\text { Std. Err. }\end{array}$ & $t$ & $P>|t|$ & [95\% Conf. & Interval] \\
\hline eltopiawa $\sim$ i & 224.218 & 1294.395 & 0.17 & 0.867 & -2760.663 & 3209.099 \\
\hline eltopia_cp & -15.08018 & 71.57016 & -0.21 & 0.838 & -180.1213 & 149.9609 \\
\hline eltopia_ḡdd & -.0714219 & .4857287 & -0.15 & 0.887 & -1.191514 & 1.048671 \\
\hline eltopia_nnd 2 & -189.1907 & 1059.312 & -0.18 & 0.863 & -2631.968 & 2253.587 \\
\hline eltopiā_cp2 & -.6127022 & 2.726427 & -0.22 & 0.828 & -6.899854 & 5.67445 \\
\hline eltopia_gdd2 & -.0000152 & .0001784 & -0.09 & 0.934 & -.0004267 & .0003962 \\
\hline eltopia_cp d & -.0129429 & .0312281 & -0.41 & 0.689 & -.0849551 & .0590693 \\
\hline eltopia_nd p & 25.72379 & 114.8015 & 0.22 & 0.828 & -239.009 & 290.4566 \\
\hline eltopia_nd d & .1435507 & .7778935 & 0.18 & 0.858 & -1.650275 & 1.937376 \\
\hline cons & -61.23941 & 396.3157 & -0.15 & 0.881 & -975.1451 & 852.6663 \\
\hline
\end{tabular}

Menomonie, WI

Linear regression

$\begin{array}{llr}\text { Number of obs } & = & 22 \\ \mathrm{~F}(9, \quad 12) & =2.10 \\ \text { Prob }>\text { F } & = & 0.1150 \\ \text { R-squared } & = & 0.7404 \\ \text { Root MSE } & = & .30863\end{array}$

\begin{tabular}{|c|c|c|c|c|c|c|}
\hline menomoniew s & Coef. & $\begin{array}{l}\text { Robust } \mathrm{HC} 3 \\
\text { Std. Err. }\end{array}$ & t & $P>|t|$ & [ $95 \%$ Conf. & Int \\
\hline menomoniew i & 45.39174 & 255.3676 & 0.18 & 0.862 & -511.0064 & 601.7899 \\
\hline menomonie_cp & .0264661 & 1.469814 & 0.02 & 0.986 & -3.175982 & 3.228915 \\
\hline menomon $\sim \bar{g}$ dd & .0114868 & .0551275 & 0.21 & 0.838 & -.1086257 & .1315993 \\
\hline menomonie i2 & -27.6094 & 167.4372 & -0.16 & 0.872 & -392.4237 & 337.2049 \\
\hline menomonie p2 & .0079874 & .0129248 & 0.62 & 0.548 & -.0201733 & .0361481 \\
\hline menomonie $\mathrm{d} 2$ & -.0000298 & .000018 & -1.65 & 0.124 & -.0000689 & $9.43 e-06$ \\
\hline menomon $\sim$ pgdd & -.0004755 & .0009211 & -0.52 & 0.615 & -.0024823 & .0015314 \\
\hline menomoni icp & -.3433426 & 1.670982 & -0.21 & 0.841 & -3.9841 & 3.297415 \\
\hline menomon igdd & .0060539 & .0579562 & 0.10 & 0.919 & -.1202218 & .1323295 \\
\hline _cons & -14.73697 & 95.96027 & -0.15 & 0.880 & -223.8164 & 194.3425 \\
\hline
\end{tabular}

Arlington, WI

Linear regression

$\begin{array}{llr}\text { Number of obs } & & 22 \\ \text { F }(9, \quad 12) & 2.30 \\ \text { Prob }>\text { F } & =0.0895 \\ \text { R-squared } & =0.7436 \\ \text { Root MSE } & =.40626\end{array}$

\begin{tabular}{|c|c|c|c|c|c|c|}
\hline arlingtonw s & Coef. & $\begin{array}{c}\text { Robust HC3 } \\
\text { Std. Err. }\end{array}$ & t & $P>|t|$ & [ $95 \%$ Conf. & Interval ] \\
\hline arlingtonw $i$ & 529.1263 & 152.3035 & 3.47 & 0.005 & 197.2855 & 860.9671 \\
\hline arlington_cp & .3725985 & 3.127428 & 0.12 & 0.907 & -6.441481 & 7.186678 \\
\hline arlingt gdd & .0838306 & .1369417 & 0.61 & 0.552 & -.2145398 & .3822009 \\
\hline arlington i2 & -336.2931 & 92.6652 & -3.63 & 0.003 & -538.1933 & -134.393 \\
\hline arlington p2 & -.0134963 & .0229302 & -0.59 & 0.567 & -.0634568 & .0364642 \\
\hline arlington $\sim \mathrm{d} 2$ & $-3.47 e-06$ & .000067 & -0.05 & 0.960 & -.0001494 & .0001424 \\
\hline arlingt pgdd & .0005176 & .0029161 & 0.18 & 0.862 & -.0058361 & .0068713 \\
\hline arlingto icp & .0699152 & 3.075955 & 0.02 & 0.982 & -6.632016 & 6.771846 \\
\hline arlingt igdd & -.1147619 & .1205757 & -0.95 & 0.360 & -.3774739 & 79501 \\
\hline _cons & -207.7053 & 72.51311 & -2.86 & 0.014 & -365.6978 & -49.71279 \\
\hline
\end{tabular}




\title{
Sellingsgrove, PA
}

\author{
Linear regression
}

$\begin{array}{lrr}\text { Number of obs } & & 22 \\ \text { F }(5, \quad 16) & 0.77 \\ \text { Prob }>\text { F } & = & 0.5824 \\ \text { R-squared } & = & 0.1930 \\ \text { Root MSE } & = & .25724\end{array}$

\begin{tabular}{|c|c|c|c|c|c|c|}
\hline sellingsgr s & Coef. & $\begin{array}{c}\text { Robust } \mathrm{HC} 3 \\
\text { Std. Err. }\end{array}$ & $t$ & $P>|t|$ & [95\% Conf. & Interval] \\
\hline selingsgro i & 42.9509 & 101.2968 & 0.42 & 0.677 & -171.7888 & 257.6906 \\
\hline selingsgro p & .70271 & .6041612 & 1.16 & 0.262 & -.5780545 & 1.983474 \\
\hline selling _gdd & (dropped) & & & & & \\
\hline sellingsg i2 & -22.14866 & 63.81773 & -0.35 & 0.733 & -157.4362 & 113.1389 \\
\hline sellingsg p2 & -.0068448 & .0063368 & -1.08 & 0.296 & -.0202783 & .0065886 \\
\hline sellingsg d2 & (dropped) & & & & & \\
\hline selling pgdd & (dropped) & & & & & \\
\hline $\begin{array}{l}\text { sellingsgr p } \\
\text { selling igdd }\end{array}$ & $\begin{array}{l}-.6320792 \\
\text { (dropped) }\end{array}$ & .6374368 & -0.99 & 0.336 & -1.983385 & .7192264 \\
\hline _cons & -18.91773 & 40.35939 & -0.47 & 0.646 & -104.4758 & 66.64037 \\
\hline
\end{tabular}

\section{Montrose, PA}

Linear regression

$\begin{array}{llr}\text { Number of obs } & = & 22 \\ \text { F } 6, & 15) & 31.57 \\ \text { Prob }>\text { F } & = & 0.0000 \\ \text { R-squared } & = & 0.5187 \\ \text { Root MSE } & =.22945\end{array}$

\begin{tabular}{|c|c|c|c|c|c|c|}
\hline montrosepa s & Coef. & $\begin{array}{l}\text { Robust } \mathrm{HC} 3 \\
\text { Std. Err. }\end{array}$ & t & $P>|t|$ & [95\% Conf. & Interval] \\
\hline montrosepa i & -89.51918 & 84.60658 & -1.06 & 0.307 & -269.8538 & 90.81547 \\
\hline montrose_cp & -.1800463 & .4105007 & -0.44 & 0.667 & -1.055008 & .6949153 \\
\hline montrose_zgdd & (dropped) & & & & & \\
\hline montrose_n 2 & 48.46891 & 46.07637 & 1.05 & 0.309 & -49.74055 & 146.6784 \\
\hline montrose_cp2 & -.0038612 & .004662 & -0.83 & 0.421 & -.0137979 & .0060755 \\
\hline montrose_g 2 & (dropped) & & & & & \\
\hline montrose_c d & -.0052577 & .0025402 & -2.07 & 0.056 & -.0106721 & .0001567 \\
\hline $\begin{array}{l}\text { montrose_n p } \\
\text { montrose_n } n \sim d\end{array}$ & $\begin{array}{r}.3784558 \\
\text { (dropped) }\end{array}$ & .3971483 & 0.95 & 0.356 & -.4680459 & 1.224957 \\
\hline - cons & 41.83163 & 38.84416 & 1.08 & 0.299 & -40.96275 & 124.626 \\
\hline
\end{tabular}




\section{REFERENCES}

AFSC (2009). Satellite Yield Insurance.

http://www.afsc.ca/Default.aspx?cid=1-984-985-1064. Accessed October, 11, 2010.

AIC (2009). Wheat Insurance Policy. http://www.aicofindia.org/. Accessed October, 11, 2010.

Atwood, J., Watts, T., Price, K., and Kastens, J. (2005) The big pictureSatellite Remote Sensing Applications in Rangeland Assessment and Crop insurance. Presented at Agricultural Outlook Forum; 24 February 2005; Arlington, VA.

Barnett, B.J. (2004). Agricultural Index Insurance products: strengths and limitations. Presented at Agricultural Outlook Forum; 19 February 2004; Arlington, VA.

Barrett, C.B., Barnett, B.J., Carter, M.R., Chantarat, S., Hansen, J.W., Mude, A.G., Osgood, D.E., Skees, J.R., Turvey, C.G. and Ward M.N. (2007) Poverty Traps and Climate Risk: Limitations and Opportunities of Index-Based Risk Financing. IRI Technical Report, No. 07-02. IRI, Columbia University, New York.

Boutton, T.W. and Tieszen, L.L. (1983). Estimation of plant biomass by spectra relectance in an East African grassland. Journal of Range Management, 36: 213-216.

Box, E.O., Holben, B.N., and Kalb, V. (1989). Accuracy of the AVHRR Vegetation Index as a Predictor of Biomass, Primary Productivity and Net CO2 Flux. Vegetatio, 80(2): 71-89.

Brown, M.E., and K.M. de Beurs, 2008. Evaluation of multi-sensor semi-arid crop season parameters based on NDVI and rainfall. Remote Sensing of Environment,112(5): 2261-2271.

Ceccato, P., Brown, M.T, Funk, C, Small, C., Holthaus, E., Siebert, A., Ward, N. (2009). Topic 8: Remote Sensing-Vegetation. Presented at Technical Issues in Index Insurance; 7-8 October 2008; IRI, Columbia University, New York. Chapter 4. 
Chantarat, S. (2009). Pro-Poor Risk Management: Essays on the Economics of Index-based Risk Transfer Prodicts. Dissertation for Cornell University. Ithaca, New York.

Davenport, M.L. and Nicholson. S.E. (1993). On the relationship between rainfall and the Normalized Difference Vegetation Index for diverse vegetation types in East Africa. International Journal of Remote Sensing 14: 2369-2389.

Di, L., Rundquist, D.C., and Han, L. (1994). Modeling relationships between $\mathrm{NDVI}$ and precipitation during vegetative growth cycles. International Journal of Remote Sensing 15: 2121-2136.

Diaz-Caneja, M.B, Conte, C.G., Catenaro, R., and Pinilla, J.F. (2009). Agricultural Insurance Schemes II Index Insurances. European Communities.

Du Plessis, W.P. (1999). Linear Regression Relationships Between NDVI vegetation and rainfall in Etosha National Park Namibia. Journal of Arid Environments, 42: 235-260.

Farrar, T.J., Nicholson, S.E., and Lare, A.R. (1994) The Influence of Soil Type on the Relationships between, NDVI, rainfall, and soil moisture in Semi-Arid Botswana. II. NDVI response to soil moisture. Remote Sensing of Environment, 50:121-133.

Fuller, D.O. (1998). Trends in NDVI time series and their relation to rangeland and cropproduction in Senegal , 1987-1993. International Journal of Remote Sensing,19(10): 2013-2018.

Hazell, P. Anderson, J., Balzer, N., Hastrup Clemmenson, A., Hess, U., Rispoli, F. (2010). Potential for scale and sustainability for weather index insurance for agriculture and rural livelihoods. IFAD WFP. Rome.

Hielkema, J.U., Prince, S.D., and Astle, W.L. (1986). Rainfall and vegetation monitoringin the Savanna Zone of the Democratic Republic of Sudan using NOAA Advance Very High Resolution Radiometer. International Journal of Remote Sensing, 7: 1499-1513.

Hellmuth, M.E., Osgood, D.E., Hess, U., Moorhead A., Bhojwani, H. (eds). (2009). Index insurance and climate risk: Prospects for development and distaster management. Climate and Society No. 2. International Research Institute for Climate and Society (IRI), Columbia University, New York, USA.

Kennedy, P. (1989). Monitoring the Vegetation of Tunisian Grazing Lands Using the Normalized Difference Vegetation Index. Ambio, 18(2):119-123. 
Lillesand, T.M. and Kiefer, R.W. (1994). Remote Sensing and Image Interpretation. New York: John Wiley, pp. 750.

Li, B., Tao, S., Dawson, R.W. (2002). Relations between AVHRR NDVI and ecoclimatic parameters in China. International Journal of Remote Sensing, 23: 989-999.

Lewis, J.E., Rowland, J., and Nadeau, A. (1998). Estimating maize production in Kenya using NDVI: some statistical considerations. International Journal of Remote Sensing, 19(13): 2609-2617.

Makaudze, E.M. and Miranda, M.J. (2009). Catastrophic Drought Insurance Based on the Remotely Sensed Normalized Difference Vegetation Index for Smallholder Farmers in Zimbabwe. Presented at the Joint $3^{\text {rd }}$ African Association of Agricultural Economists, Cape Town, South Africa, September 19-23, 2010.

Malo, A.R. and Nicholson, S.E. (1990). A study of rainfall and vegetation dynamics in the African Sahel using Normalized Difference Vegetation Index. Journal of Arid Environments, 19:1-24.

Mkhabela, M.S., Mkhabela, M.S., Mashinini, N.N.(2005). Early maize yield forecasting in the four agro-ecological regions of Swaziland using NDVI data derived from NOAA's-AVHRR. Agricultural and Forest Meteorology,129:1-9

Norton, M. and Turvey, C.G. (2007) WeatherWizard. www.weatherwizard.us

Pareulo, J. M. and Lauenroth, W. K. (1995). Regional patterns of normalized difference vegetation index in north American shrublands and grasslands. Ecology, 76(6):1888-1898.

Prince, S.D. and Tucker, C.J. (1986). Satellite remote sensing of rangelands in Botswana II. NOAA AVHRR and herbaceous vegetation. International Journal of Remote Sensing, 7:1555-1570.

Richard, Y. and Poccard, I. (1998). A statistical study of NDVI sensitivity to seasonal and interannual rainfall variations in southern Africa. International Journal of Remote Sensing, 19(15): 2907-2920.

Rowley, R.J., Price, K.P., Kastens, J. (2007). Remote Sensing and the Rancher: Linking Rancher Perception and Remote Sensing. Rangeland Ecology and Management, 6: 359-368. 
Skees, J.R. (2008) Innovations in Index Insurance for the Poor in Lower Income Countries. Agricultural and Resource Economics Review. 37(1)1-15.

Syroka, J. (2006). Weather Risk Management for Agriculture. Risk Management in Agriculture for Natural Hazards. ISMEA. December 2006.

Tan, S.Y. (2007). The influence of temperature and precipitation climate regimes on vegetation dynamics in the US Great Plains: a satellite bioclimatology case study. International Journal of Remote Sensing, 28(22):4947-4966.

Thoma, D.P., Bailey, D.W., Long, D.S., Neilson, G.A., Henry, M.P., Breneman, M.C., and Montagne, C. (2002). Journal of Range Management, 55(4):383389.

Tieszen, L.L., Reed, B.C., Bliss, N.B, Wylie, B.K., and Dejong, D.D. (1997). NDVI, C3 and C4 Production, and Distributions in Great Plains Grassland Land Cover Classes. Ecological Applications, 7(1): 59-78.

Tucker, C.J., Vanpraet, C.L., Sharman, M.J. and van Itternsum, G. (1985). Relationship between atmospheric $\mathrm{CO} 2$ variations and a satellite-derived vegetation index. Remote Sensing of Environment, 17: 233-249.

Udelhoven , T., Stellmes, M., del Barrio, G, and Hill, J. (2009). Assessment of rainfall and NDVI anomalies in Spain (1989-1999) using distributed lag models. International Journal of Remote Sensing, 30(8): 1961-1976 .

USDA (2009). A Risk Management Agency Factsheet Pasture, Rangeland, and Forage Pilot Insurance Program. January 2009. http://www.rma.usda.gov/pubs/rme/PRFfactsheet.pdf

Wang, J., Price, K.P., and Rich, P.M. (2001). Spatial patterns of NDVI in response to precipitation and temperature in the central Great Plains. International Journal of Remote Sensing, 22(18): 3827-3844.

Wang, J., Rich, P.M., Price, K.P. (2003). Temporal responses of NDVI to precipitation and temperature in the central Great Plains, USA. International Journal of Remote Sensing, 24 (11): 2345-2364.

Yang L., Wylie, B., Tieszen, L.L., Reed, B.C. (1998). An Analysis of Relationships among Climate Forcing and Time-Integrated NDVI of Grasslands over the U.S. Northern and Central Great Plains. Remote Sensing Environment, 65: 25-37 
Zhou Liming et al. (2001). Variations in northern vegetation activity inferred from satellite data of vegetation index during 1981 to 1999. Journal of Geophysical Research,106(D17): 20069-20083. 